\title{
Syntheses of Benzofuranoquinolines and Analogues via Photoinduced Acceptorless Dehydrogenative Annulation of o-Phenyl-furanylpyridines
}

Jinming Fan ${ }^{\dagger}$, Wei Zhang ${ }^{\dagger}$, Wangxi Gao, Tao Wang, Wei-Liang Duan, Yong Liang ${ }^{\ddagger}$, Zunting Zhang*

Key Laboratory of Applied Surface and Colloid Chemistry (Ministry of Education), School of Chemistry and Chemical Engineering, Shaanxi Normal University, Xi'an 710119, People's Republic of China 


\section{Contents}

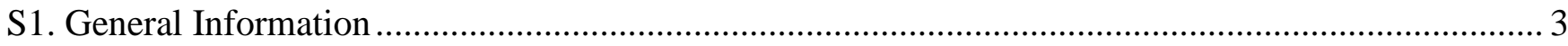

S2. General procedure for the preparation of Bromophenylpyridines .............................................. 3

S3. General procedure for the preparation of Phenyl-furanyl/thienylPyridines and Pyrimidines............. 4

S4. General procedure for the preparation of Benzofurano/thienoquinolines and Analogues.................. 5

S5. Gram scale reaction : Synthesis of 6-Methylbenzo[h]thieno[2,3-f]quinoline-9-carbonitrile (2j)

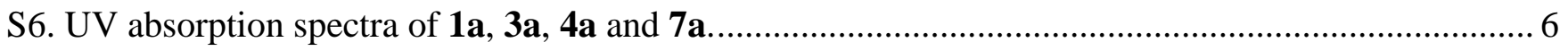

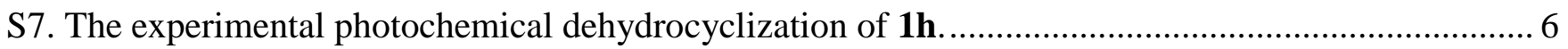

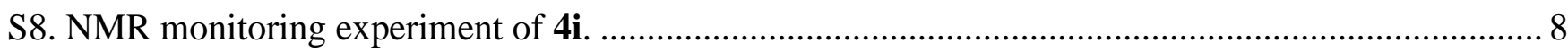

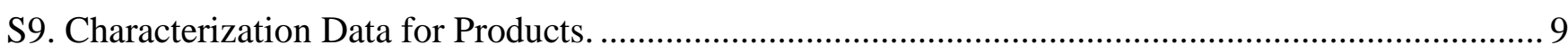

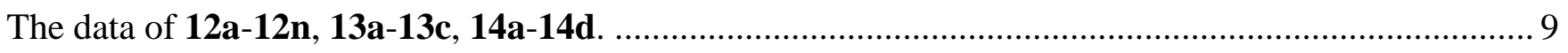

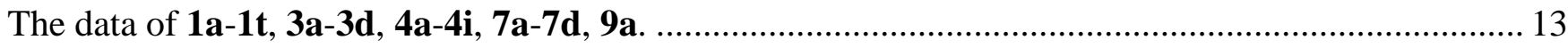

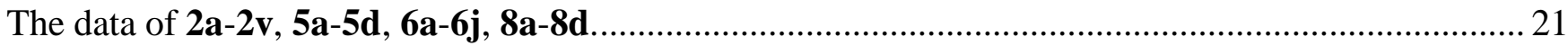

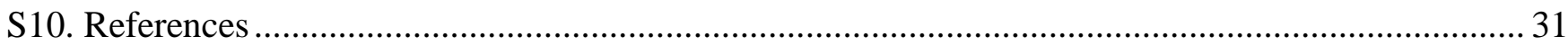

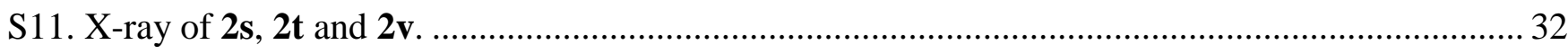

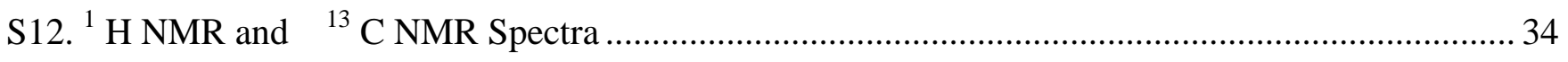

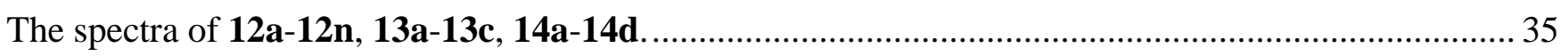

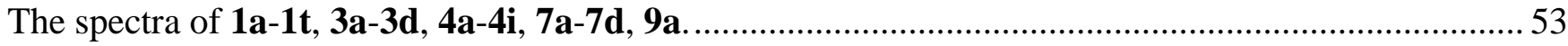

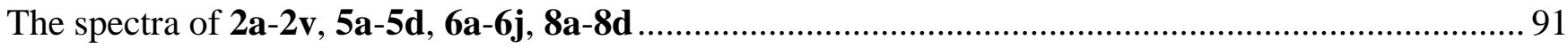




\section{S1. General Information}

${ }^{1} \mathrm{H}$ NMR and ${ }^{13} \mathrm{C}$ NMR were recorded on a Bruker-400 MHz, $600 \mathrm{MHz}$ Spectrometer $\left({ }^{1} \mathrm{H}: 400 \mathrm{MHz}\right.$, $\left.{ }^{13} \mathrm{C}: 100 \mathrm{MHz}\right),\left({ }^{1} \mathrm{H}: 600 \mathrm{MHz},{ }^{13} \mathrm{C}: 150 \mathrm{MHz}\right)$, using $\mathrm{CDCl}_{3}$, DMSO- $d_{6}$ and $\mathrm{D}_{2} \mathrm{O}$ as the solvent at room temperature. The chemical shifts $(\delta)$ were expressed in ppm and the coupling constants $(J)$ were expressed in Hz. High-resolution mass spectra (HRMS) were recorded on a Bruker MAXIS spectrometer. IR spectra were recorded on a Bruker Tensor 27 spectrometer. All the irradiation experiments were performed in a BL-GHX-V photo-chemical reactor equipped with a $500 \mathrm{~W}$ high-pressure mercury lamp.

\section{S2. General procedure for the preparation of Bromophenylpyridines}

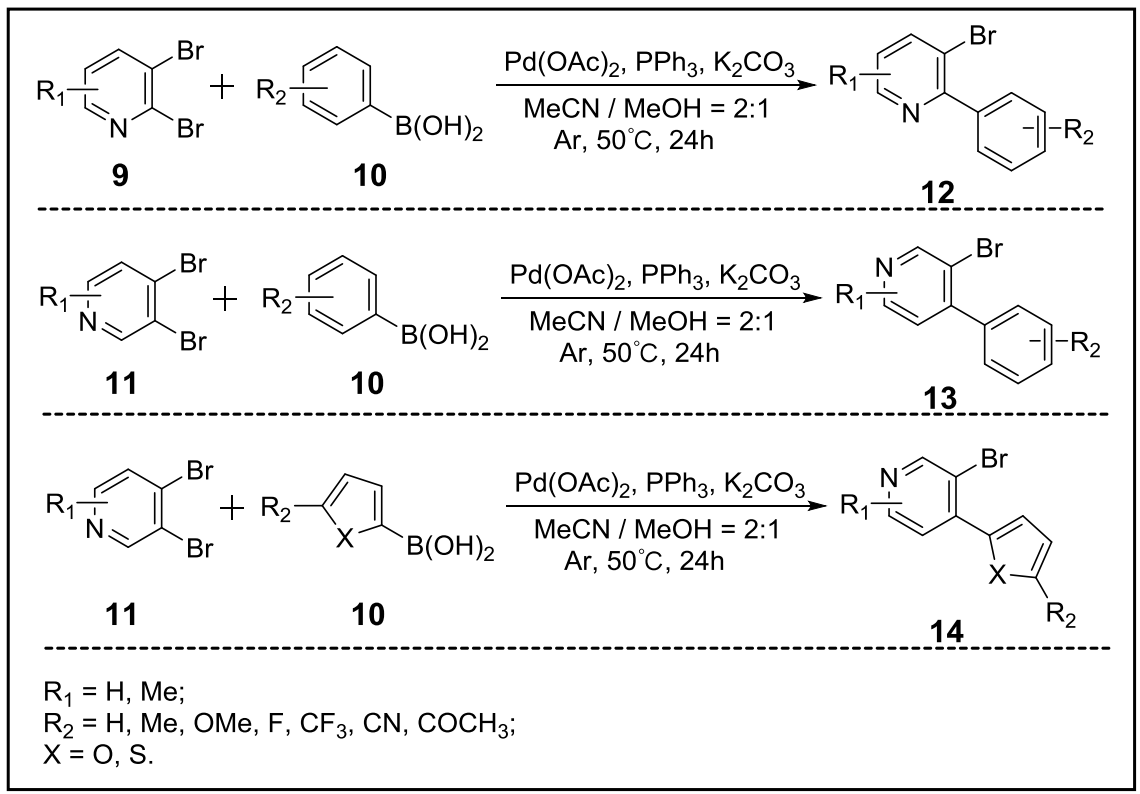

Scheme S1 Preparation of Bromophenylpyridines (12-14)

A mixture of 2,3-dibromopyridine 9 or 3,4-dibromopyridine 11 (3 mmol, $708 \mathrm{mg}$ ), arylboronic acid 10 (1.1 eq, $3.3 \mathrm{mmol}), \mathrm{PPh}_{3}(20 \%, 0.6 \mathrm{mmol}, 157 \mathrm{mg}), \mathrm{K}_{2} \mathrm{CO}_{3}(2 \mathrm{eq}, 6 \mathrm{mmol}, 828 \mathrm{mg})$ and $\mathrm{Pd}(\mathrm{OAc})_{2}(10 \%$, $0.3 \mathrm{mmol}, 68 \mathrm{mg})$ were dissloved in $\mathrm{MeCN}: \mathrm{MeOH}=2: 1(20 \mathrm{ml}: 10 \mathrm{ml})$. The mixture was flushed with argon, sealed, and stirred at $50{ }^{\circ} \mathrm{C}$ for $24 \mathrm{~h}$ in an oil bath. Then the reaction mixture was cooled at room temperature and filtered through Celite. The filtrate was concentrated under reduced pressure. The residue was dissolved in $\mathrm{CH}_{2} \mathrm{Cl}_{2}(40 \mathrm{~mL} \times 3)$ and washed with water and dried over $\mathrm{Na}_{2} \mathrm{SO}_{4}$. Combined organic layers were evaporated under reduced pressure, and the residue was purified by column chromatography on silica (EtOAc $-\mathrm{Hex}=1: 30 \sim 1: 20$ ) to provide pure bromoarylpyridines $\mathbf{1 2}, \mathbf{1 3}$ and 14. The synthesis procedures of compounds were carried out according to the known methods. ${ }^{[1]}$ 


\section{S3. General procedure for the preparation of $o$-phenyl-furanyl/thienylpyridines and pyrimidines}

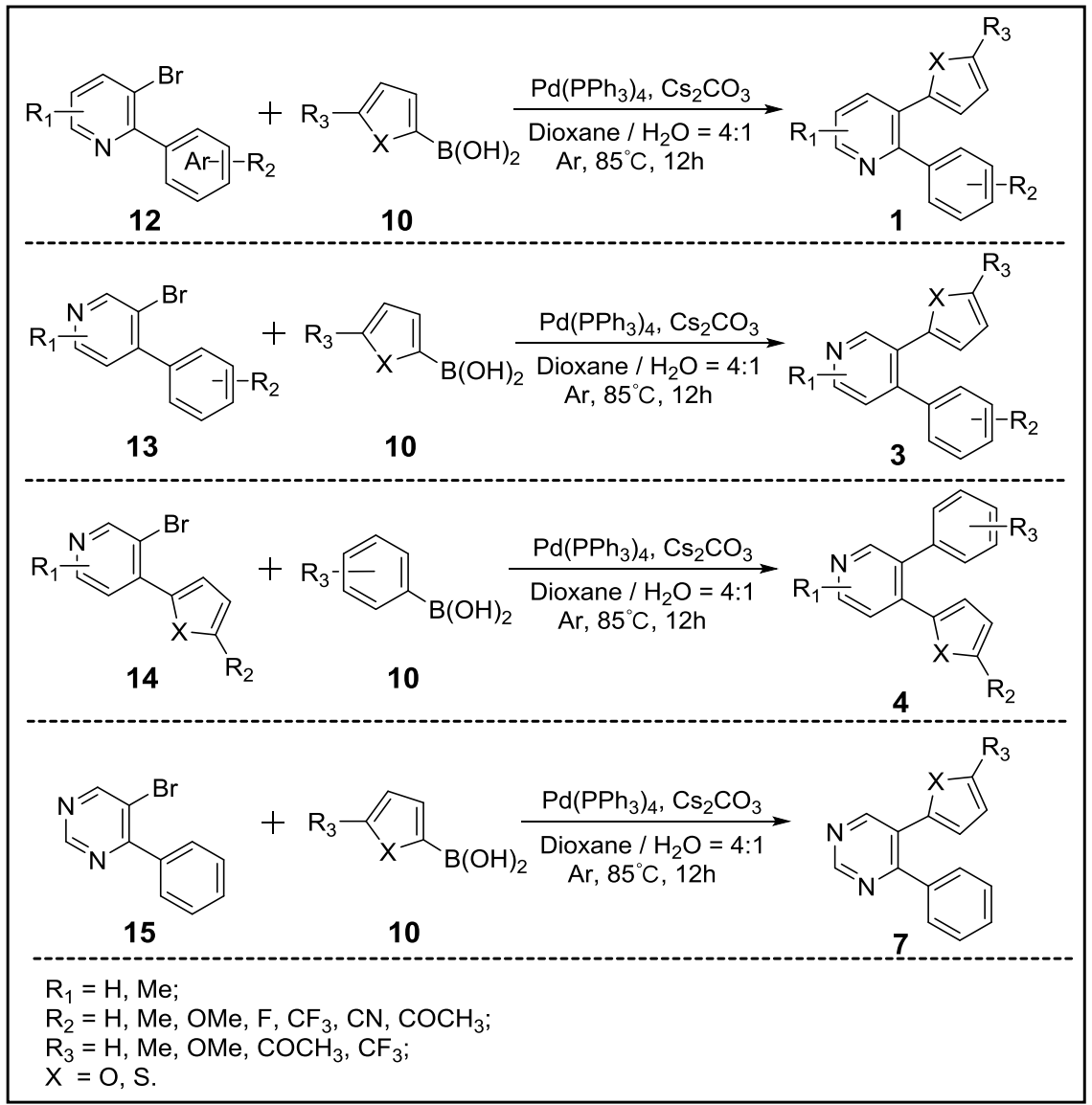

Scheme S2 Preparation of $o$-phenyl-furanyl/thienylpyridines and pyrimidines $1,3,4,7$

The mixture of bromoarylpyridines 12 ( $1 \mathrm{mmol})$, arylboronic acid 10 (3 eq, $\left.3 \mathrm{mmol}), \mathrm{Pd}_{(\mathrm{PPh}}\right)_{4}(5 \%$ mmol, $58 \mathrm{mg}$ ) and $\mathrm{Cs}_{2} \mathrm{CO}_{3}(5 \mathrm{eq}, 5 \mathrm{mmol}, 1.63 \mathrm{~g}$ ) was dissolved in a mixed solvent composed of dioxane : $\mathrm{H}_{2} \mathrm{O}=4: 1(12 \mathrm{ml}: 3 \mathrm{ml})$. The reaction mixture was allowed to stir at $85{ }^{\circ} \mathrm{C}$ for $6-12 \mathrm{~h}$ under Ar. Then, the mixture was poured into the water and extracted with ethyl acetate $(30 \mathrm{~mL} \times 3)$. The combined organic layers were dried over $\mathrm{Na}_{2} \mathrm{SO}_{4}$ and concentrated under reduced pressure. The residue was purified by column chromatography (EtOAc - Hex $=1: 40 \sim 1: 30$ ) to give the desired product $o$-phenyl-furanyl/thienylpyridines 1 . The synthesis procedures of compounds $\mathbf{1}, \mathbf{3}, \mathbf{4}$ and $\mathbf{7}$ were carried out according to the known methods. ${ }^{[2]}$ 


\section{S4. General procedure for the Synthesis of Benzofurano/thienoquinolines and Analogues}

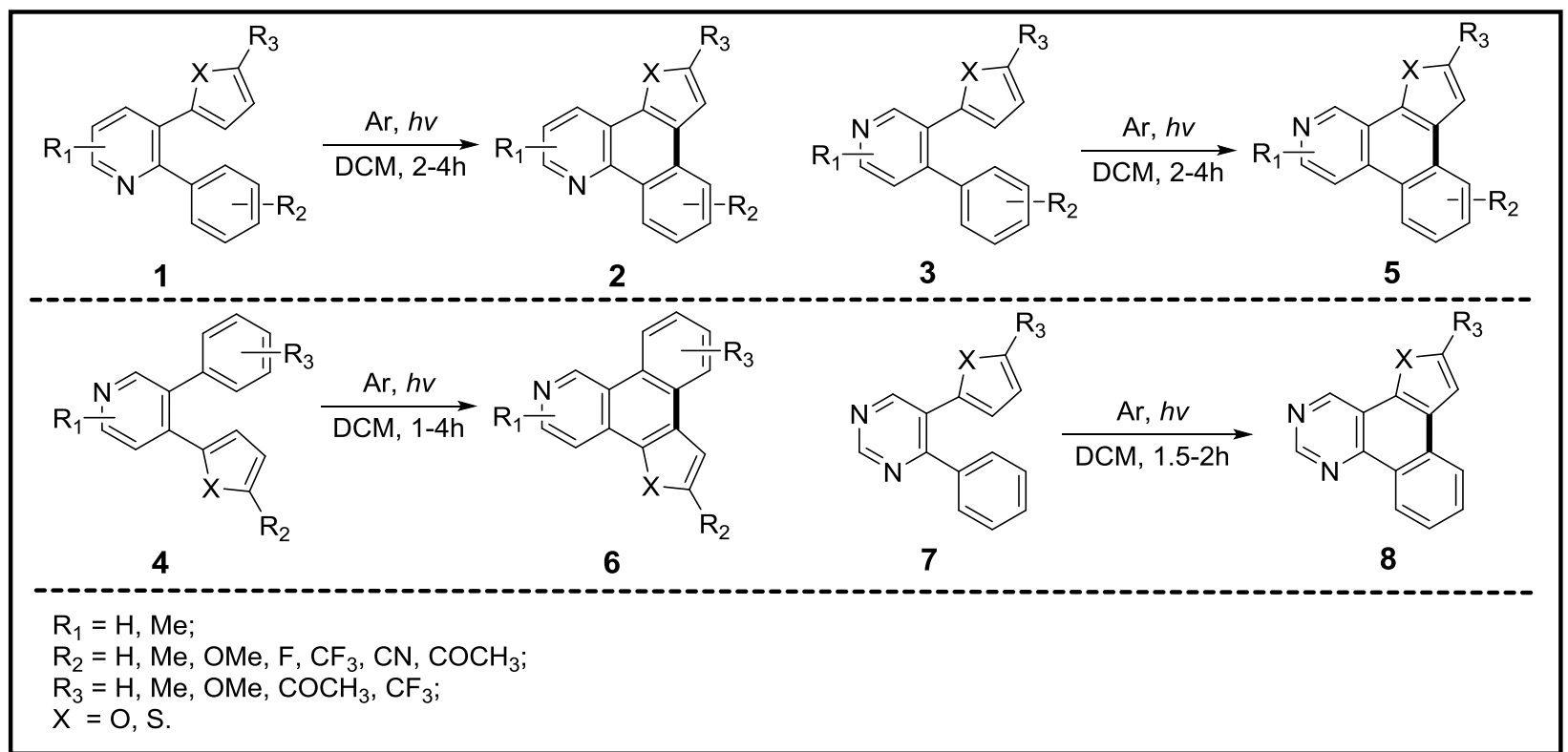

Scheme S3 Synthesis of Benzofurano/thienoquinolines and Analogues 2, 5, 6, 8

$o$-Phenyl-furanyl/thienylpyridines $1(0.25 \mathrm{mmol})$ was added to DCM $(50 \mathrm{~mL}, 5 \mathrm{mM})$. The solution was contained in a $100 \mathrm{~mL}$ quartz tube, degassed (ultrasound) for $30 \mathrm{~min}$, deaerated by bubbling argon for 30 min and the quartz tube was sealed by parafilm; irradiation in a BL-GHX-V photo-chemical reactor at room temperature until reactant was consumed completely as indicated by thin-layer chromatography (TLC). The solvent was removed under reduced pressure, and the residue was purified by column chromatography (EtOAc - Hex $=1: 30 \sim 1: 10)$ to get Benzofurano/thienoquinolines 2.

\section{S5. Gram scale reaction: synthesis of 6-methylbenzo[ $h]$ thieno[2,3-f]quinoline-9- carbonitrile $(2 \mathbf{j})$}

4-(3-(5-methylthiophen-2-yl)pyridin-2-yl)benzonitrile $\mathbf{1 j}$ (276 mg, $1 \mathrm{mmol}$ ) was added to DCM (200 $\mathrm{mL}, 5 \mathrm{mM}$ ). The solution was contained in $200 \mathrm{~mL}$ quartz tube, degassed (ultrasound) for $30 \mathrm{~min}$, deaerated by bubbling argon for 30 min three times and the quartz tube was sealed by parafilm, and irradiated in a BL-GHX-V photo-chemical reactor at room temperature for $2 \mathrm{~h}$, until $\mathbf{1 j}$ was consumed completely as indicated by thin-layer chromatography (TLC). The solvent was removed under reduced pressure, and the residue was purified by column chromatography (ethyl acetate: hexane $=1: 10$ ) to get 6-methylbenzo[ $h]$ thieno[2,3-f]quinoline-9-carbonitrile $\mathbf{2 j}$ (75\%, $206 \mathrm{mg}$ ). 


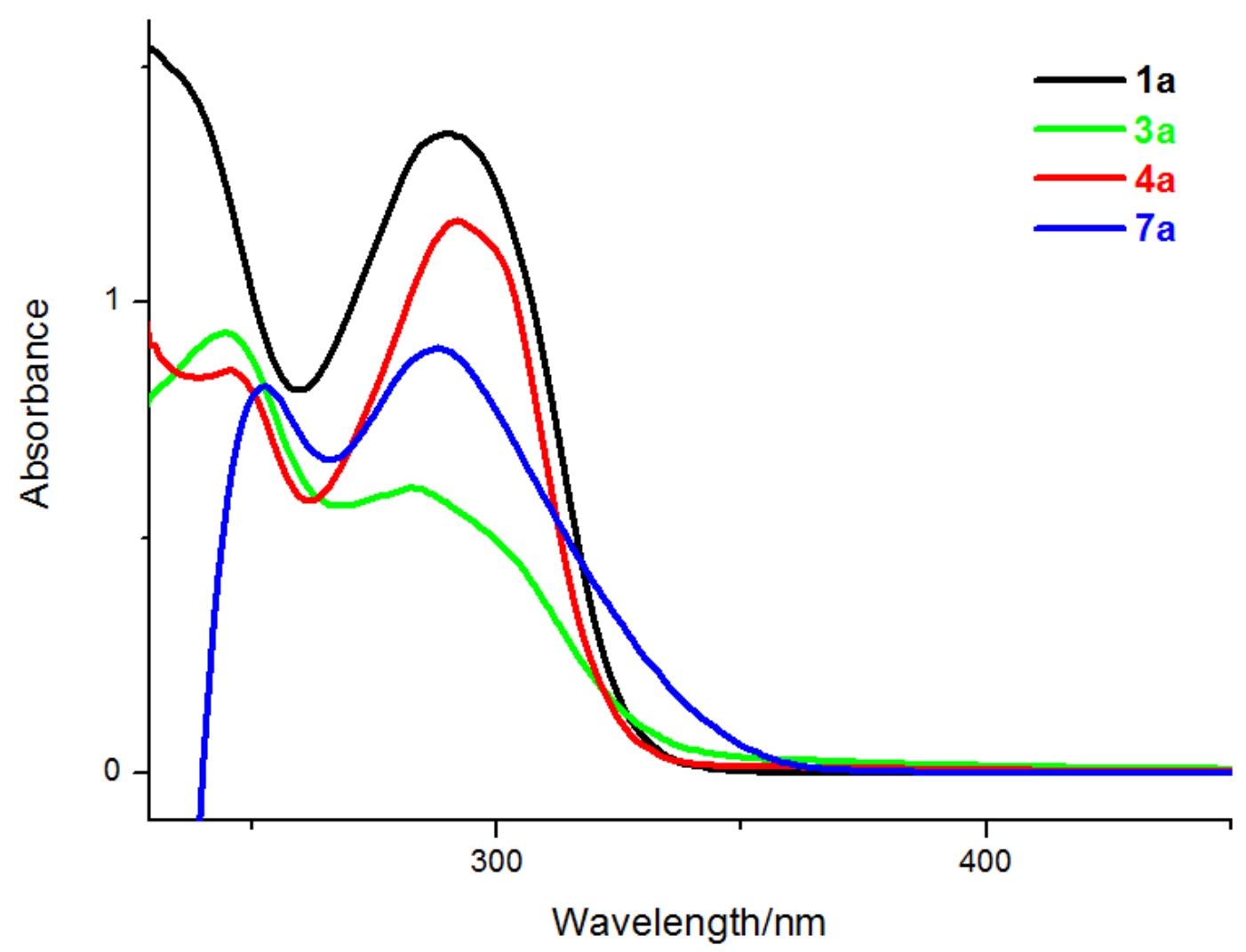

Figure S1. UV absorption spectra of 1a, 3a, $4 \mathbf{a}$ and $7 \mathbf{a}$ in $\operatorname{DCM}\left(10^{-5} \mathrm{M}\right)$.

\section{S7. $\mathrm{H}_{2}$ detection for the photoinduced dehydrogenative annulation of $1 \mathrm{~h}$ by GC}

GC conditions: Argon was used as carrier gas, stainless steel column (column length $2 \mathrm{~m}$, column temperature at $50^{\circ} \mathrm{C}$, Tam TDS-01 60 80 mesh) and thermal conductivity detector (TCD temperature at $80{ }^{\circ} \mathrm{C}$ ) were used for gas chromatography analysis. Under the conditions of gas velocity of $0.5 \mathrm{Mpa}$ and the flow rate of $70 \mathrm{~mL} / \mathrm{min}$, gas was analysized at room temperature with injection of $30 \mathrm{uL}$.

Experiment of photoinduced dehydrogenative annulation of 1h: In order to remove the oxygen from the solvent, DCM (200 ml) was degassed for an hour by ultrasonic and sodium sulfite $(25 \mathrm{~g})$ and hydroquinone $(2.0 \mathrm{~g})$ were added and the mixture was refluxed for $2 \mathrm{~h}$. Then, 4-(3-(furan-2-yl)pyridin2-yl)benzonitrile $\mathbf{1 h}(61.5 \mathrm{mg}, 0.25 \mathrm{mmol})$ was dissolved in $50 \mathrm{~mL}$ deoxidization DCM in a $100 \mathrm{~mL}$ quartz tube and conducted in argon atmosphere. After sealing, the quartz tube was irradiated with a high-pressure mercury lamp (500 W) for $4 \mathrm{~h}$.

Results of detection: After the reaction of photoinduced dehydrogenative annulation for $\mathbf{1 h}$, gas in the quartz tube was detected by GC. The retention time tR1 of standard $\mathrm{H}_{2}$ sample was 1.469 min and the retention time $\mathrm{tR}_{2}$ of gas in the quartz tube was 1.506 min (Figure S2). It indcated that there was generated $\mathrm{H}_{2}$ in the process of photoinduced $\mathbf{1 h}$. 


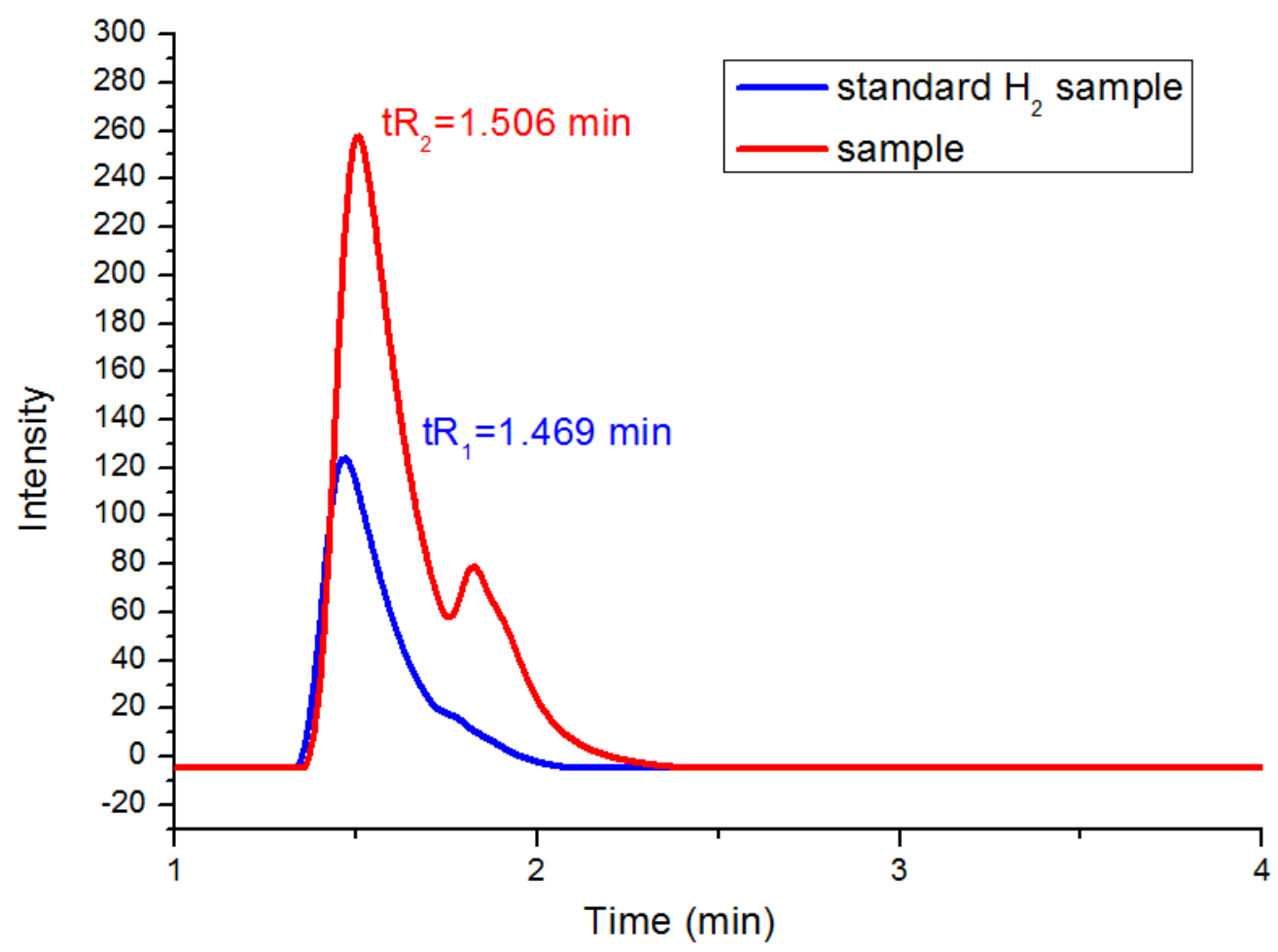

Figure S2. Chromatogram of reference substance $\mathrm{H}_{2}$ and gas in the tube of photoinduced dehydrogenative annulation for $\mathbf{1 h}$. 

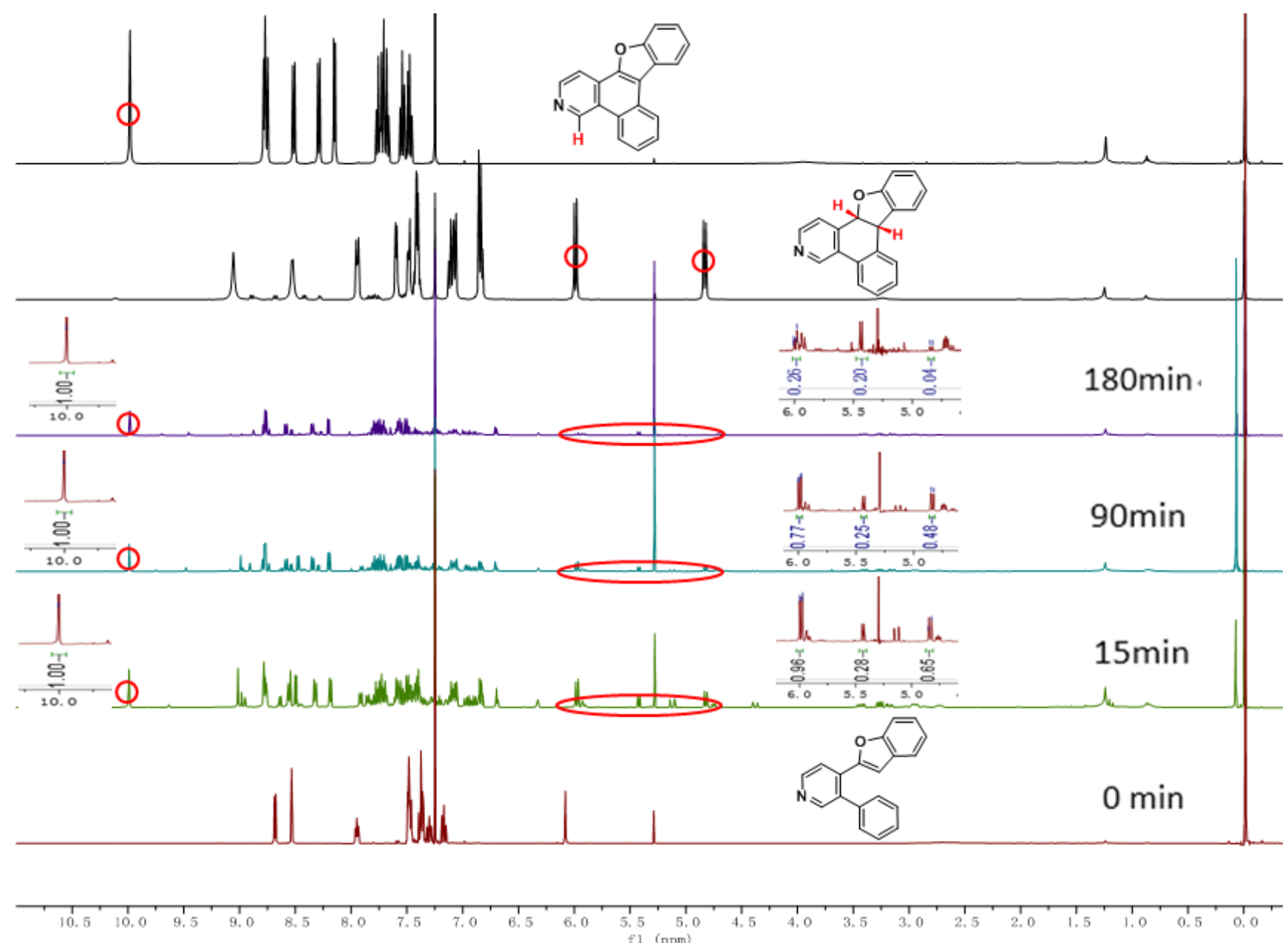

Figure S3. ${ }^{1} \mathrm{H}$ NMR spectral change conversion of $4 \mathbf{i}$ to $\mathbf{6 j}$ and $\mathbf{6 i}$ in $\mathrm{CDCl}_{3}$ under UV irradiation for diffrent time. The representative peaks for each species are ${ }^{1} \mathrm{H}$ NMR spectra of irradiation $4 \mathbf{i}$ for $0 \mathrm{~min}, 15 \mathrm{~min}, 90 \mathrm{~min}, 180 \mathrm{~min}$ and $\mathbf{6 j}$, $\mathbf{6 i}$ from bottom to top. 


\section{S9. Characterization Data for Products}

The data of 12a-12n, 13a-13c, 14a-14d

\section{3-Bromo-2-phenylpyridine (12a)}

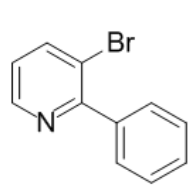

Yield: 70\% (490 mg). Colorless oil. Purified by flash column chromatography (EtOAc/ hexane $=1 / 30) .{ }^{1} \mathrm{H}$ NMR $\left(400 \mathrm{MHz}, \mathrm{CDCl}_{3}\right) \delta 8.62(\mathrm{dd}, J=4.7,0.9 \mathrm{~Hz}, 1 \mathrm{H}), 7.97(\mathrm{dd}, J$ $=8.1,1.0 \mathrm{~Hz}, 1 \mathrm{H}), 7.73-7.65(\mathrm{~m}, 2 \mathrm{H}), 7.50-7.41(\mathrm{~m}, 3 \mathrm{H}), 7.10(\mathrm{dd}, J=8.0,4.6 \mathrm{~Hz}$, $1 \mathrm{H}){ }^{[1]}$

\section{3-Bromo-2-(p-tolyl)pyridine (12b)}

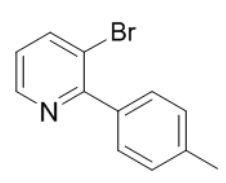

Yield: $72 \%$ (533 mg). Colorless oil. Purified by flash column chromatography (EtOAc/ hexane = 1/30). ${ }^{1} \mathrm{H}$ NMR $\left(400 \mathrm{MHz}, \mathrm{CDCl}_{3}\right) \delta 8.61(\mathrm{dd}, J=4.6,1.5 \mathrm{~Hz}, 1 \mathrm{H}), 7.96(\mathrm{dd}$, $J=8.0,1.4 \mathrm{~Hz}, 1 \mathrm{H}), 7.61(\mathrm{~d}, J=8.1 \mathrm{~Hz}, 2 \mathrm{H}), 7.28(\mathrm{~d}, J=7.9 \mathrm{~Hz}, 2 \mathrm{H}), 7.09$ (dd, $J=8.0$, $4.6 \mathrm{~Hz}, 1 \mathrm{H}), 2.42(\mathrm{~s}, 3 \mathrm{H}){ }^{[3]}$

\section{3-Bromo-2-(4-methoxyphenyl)pyridine (12c)}

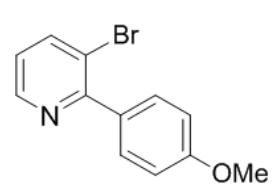

Yield: $71 \%$ (560 mg). Colorless oil. Purified by flash column chromatography $($ EtOAc/hexane $=1 / 30) .{ }^{1} \mathrm{H} \mathrm{NMR}\left(400 \mathrm{MHz}, \mathrm{CDCl}_{3}\right) \delta 8.58(\mathrm{dd}, J=4.6,1.4 \mathrm{~Hz}, 1 \mathrm{H})$, $7.93(\mathrm{dd}, J=8.0,1.4 \mathrm{~Hz}, 1 \mathrm{H}), 7.70-7.64(\mathrm{~m}, 2 \mathrm{H}), 7.05(\mathrm{dd}, J=8.0,4.6 \mathrm{~Hz}, 1 \mathrm{H})$, $7.00-6.95(\mathrm{~m}, 2 \mathrm{H}), 3.84(\mathrm{~s}, 3 \mathrm{H}){ }^{[1]}$

\section{3-Bromo-2-(4-fluorophenyl)pyridine (12d)}

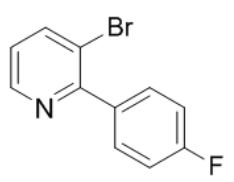

Yield: 52\% (390 mg). White solid. m.p. 89.0-89.6 ${ }^{\circ} \mathrm{C}$. Purified by flash column chromatography $($ EtOAc/hexane $=1 / 30) .{ }^{1} \mathrm{H}$ NMR $\left(400 \mathrm{MHz}, \mathrm{CDCl}_{3}\right) \delta 8.60(\mathrm{~d}, J=4.5$ $\mathrm{Hz}, 1 \mathrm{H}), 7.97(\mathrm{~d}, J=8.1 \mathrm{~Hz}, 1 \mathrm{H}), 7.68(\mathrm{dd}, J=7.4,5.8 \mathrm{~Hz}, 2 \mathrm{H}), 7.15(\mathrm{~d}, J=8.6 \mathrm{~Hz}$, 2H), $7.11(\mathrm{~d}, J=4.3 \mathrm{~Hz}, 1 \mathrm{H}){ }^{[1]}$

\section{1-(4-(3-Bromopyridin-2-yl)phenyl)ethan-1-one (12e)}

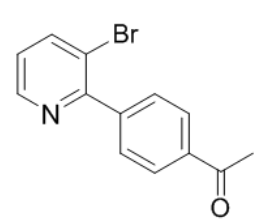

Yield: 53\% (437 mg). White solid. m.p. 66.3-67.4 ${ }^{\circ} \mathrm{C}$. Purified by flash column chromatography $($ EtOAc/hexane $=1 / 30) .{ }^{1} \mathrm{H}$ NMR $\left(400 \mathrm{MHz}, \mathrm{CDCl}_{3}\right) \delta 8.64(\mathrm{dd}, J=$ 4.6, 1.2 Hz, 1H), $8.05(\mathrm{~d}, J=8.2 \mathrm{~Hz}, 2 \mathrm{H}), 8.00(\mathrm{dd}, J=8.1,1.2 \mathrm{~Hz}, 1 \mathrm{H}), 7.78(\mathrm{~d}, J=$ $8.2 \mathrm{~Hz}, 2 \mathrm{H}), 7.18(\mathrm{dd}, J=8.1,4.6 \mathrm{~Hz}, 1 \mathrm{H}), 2.65(\mathrm{~s}, 3 \mathrm{H}) .{ }^{13} \mathrm{C} \mathrm{NMR}\left(101 \mathrm{MHz}, \mathrm{CDCl}_{3}\right)$ $\delta 197.8,157.2$, 148.4, 144.1, 141.6, 137.1, 129.8, 128.1, 123.9, 119.9, 26.9. IR (KBr), v $\left(\mathrm{cm}^{-1}\right) 3697$, 3033, 1778, 1679, 1269, 1006, 792, 595; HRMS (ESI) m/z calcd for. $\mathrm{C}_{13} \mathrm{H}_{10} \mathrm{BrNO}[\mathrm{M}+\mathrm{Na}]^{+} 297.9838$, found 297.9831 .

\section{4-(3-Bromopyridin-2-yl)benzonitrile (12f)}

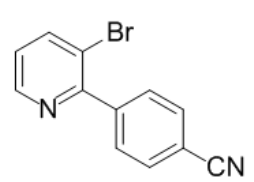

Yield: $62 \%$ (480 mg). White solid. m.p. 110.6-111.5 ${ }^{\circ} \mathrm{C}$. Purified by flash column chromatography $($ EtOAc/hexane $=1 / 20) .{ }^{1} \mathrm{H}$ NMR $\left(400 \mathrm{MHz}, \mathrm{CDCl}_{3}\right) \delta 8.68-8.61$ $(\mathrm{m}, 1 \mathrm{H}), 8.02(\mathrm{~d}, J=8.2 \mathrm{~Hz}, 1 \mathrm{H}), 7.81(\mathrm{~d}, J=8.3 \mathrm{~Hz}, 2 \mathrm{H}), 7.75(\mathrm{~d}, J=8.2 \mathrm{~Hz}, 2 \mathrm{H})$, 
$7.21(\mathrm{dd}, J=8.1,4.7 \mathrm{~Hz}, 1 \mathrm{H}) .{ }^{13} \mathrm{C}$ NMR $\left(101 \mathrm{MHz}, \mathrm{CDCl}_{3}\right) \delta 156.3,148.5,143.9,141.7,131.9,130.3$, 124.3, 119.8, 118.8, 112.6; IR (KBr), $v\left(\mathrm{~cm}^{-1}\right)$ 3749, 3045, 2227, 1728, 1429, 1298, 1014, 796; HRMS (ESI) $\mathrm{m} / \mathrm{z}$ calcd for. $\mathrm{C}_{12} \mathrm{H}_{7} \mathrm{BrN}_{2}[\mathrm{M}+\mathrm{H}]^{+} 258.9865$, found 258.9861 .

\section{3-Bromo-2-(4-(trifluoromethyl)phenyl)pyridine (12g)}

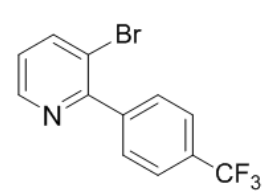

Yield: $68 \%$ (614 mg). Yellow solid. m.p. 50.5-51.8 ${ }^{\circ} \mathrm{C}$. Purified by flash column chromatography $($ EtOAc/hexane $=1 / 30) .{ }^{1} \mathrm{H}$ NMR $\left(600 \mathrm{MHz}, \mathrm{CDCl}_{3}\right) \delta 8.64(\mathrm{dd}, J=$ 4.6, 1.4 Hz, 1H), $8.01(\mathrm{dd}, J=8.1,1.4 \mathrm{~Hz}, 1 \mathrm{H}), 7.81(\mathrm{~d}, J=8.1 \mathrm{~Hz}, 2 \mathrm{H}), 7.73(\mathrm{~d}, J=$ $8.2 \mathrm{~Hz}, 2 \mathrm{H}), 7.18(\mathrm{dd}, J=8.1,4.6 \mathrm{~Hz}, 1 \mathrm{H}) .^{[1]}$

\section{3-(3-Bromopyridin-2-yl)benzonitrile (12h)}

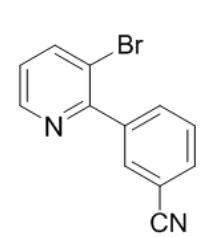

Yield: 41\% (317 mg). White solid. m.p. 87.5-88.8 ${ }^{\circ} \mathrm{C}$. Purified by flash column chromatography $($ EtOAc/hexane $=1 / 20) .{ }^{1} \mathrm{H}$ NMR $\left(600 \mathrm{MHz}, \mathrm{CDCl}_{3}\right) \delta 8.64(\mathrm{dd}, J=4.6$, $1.4 \mathrm{~Hz}, 1 \mathrm{H}), 8.11-7.97(\mathrm{~m}, 2 \mathrm{H}), 7.96-7.92(\mathrm{~m}, 1 \mathrm{H}), 7.73-7.69(\mathrm{~m}, 1 \mathrm{H}), 7.57(\mathrm{t}, J=$ $7.8 \mathrm{~Hz}, 1 \mathrm{H}), 7.21(\mathrm{dd}, J=8.1,4.6 \mathrm{~Hz}, 1 \mathrm{H}) .{ }^{13} \mathrm{C} \mathrm{NMR}\left(151 \mathrm{MHz}, \mathrm{CDCl}_{3}\right) \delta 155.8,148.5$, $141.7,140.8,133.9,133.3,132.3,129.0,124.3,119.8,118.6,112.5$. IR (KBr), $v\left(\mathrm{~cm}^{-1}\right) 3706,3043$, 2225, 1739, 1429, 1020, 796; HRMS (ESI) $\mathrm{m} / \mathrm{z}$ calcd for. $\mathrm{C}_{12} \mathrm{H}_{7} \mathrm{BrN}_{2}[\mathrm{M}+\mathrm{H}]^{+} 258.9865$, found 258.9865 .

\section{1-(3-(3-Bromopyridin-2-yl)phenyl)ethan-1-one (12i)}

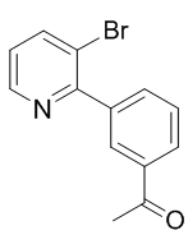

Yield: 49\% (404 mg). White solid. m.p. 68.8-70.4 ${ }^{\circ} \mathrm{C}$. Purified by flash column chromatography $($ EtOAc/hexane $=1 / 30) .{ }^{1} \mathrm{H} \mathrm{NMR}\left(400 \mathrm{MHz}, \mathrm{CDCl}_{3}\right) \delta 8.68-8.58(\mathrm{~m}$, $1 \mathrm{H}), 8.27(\mathrm{~s}, 1 \mathrm{H}), 8.01(\mathrm{dd}, J=12.6,4.4 \mathrm{~Hz}, 2 \mathrm{H}), 7.89(\mathrm{~d}, J=7.7 \mathrm{~Hz}, 1 \mathrm{H}), 7.56(\mathrm{t}, J=7.8$

$\mathrm{Hz}, 1 \mathrm{H}), 7.17(\mathrm{dd}, J=8.1,4.7 \mathrm{~Hz}, 1 \mathrm{H}), 2.64(\mathrm{~s}, 3 \mathrm{H}) \cdot{ }^{13} \mathrm{C} \mathrm{NMR}\left(101 \mathrm{MHz}, \mathrm{CDCl}_{3}\right) \delta$ 197.8, 157.3, 148.4, 141.6, 140.1, 137.1, 134.0, 129.7, 128.5, 128.5, 123.8, 119.9, 26.8. IR (KBr), $v$ $\left(\mathrm{cm}^{-1}\right)$ 3448, 2993, 1915, 1681, 1355, 1242, 1012, 690, 592; HRMS (ESI) m/z calcd for. $\mathrm{C}_{13} \mathrm{H}_{10} \mathrm{BrNO}$ $[\mathrm{M}+\mathrm{H}]^{+}$276.0019, found 276.0016 .

\section{3-Bromo-2-(naphthalen-2-yl)pyridine (12j)}

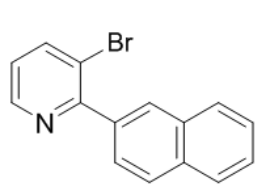

Yield: 60\% (509 mg). Yellow oil. Purified by flash column chromatography (EtOAc/ hexane = 1/30). ${ }^{1} \mathrm{H}$ NMR $\left(400 \mathrm{MHz}, \mathrm{CDCl}_{3}\right) \delta 8.67(\mathrm{dd}, J=4.6,1.4 \mathrm{~Hz}, 1 \mathrm{H}), 8.24(\mathrm{~s}$, $1 \mathrm{H}), 8.00(\mathrm{dd}, J=8.1,1.4 \mathrm{~Hz}, 1 \mathrm{H}), 7.98-7.89(\mathrm{~m}, 3 \mathrm{H}), 7.85(\mathrm{dd}, J=8.5,1.6 \mathrm{~Hz}$, $1 \mathrm{H}), 7.57-7.51(\mathrm{~m}, 2 \mathrm{H}), 7.12(\mathrm{dd}, J=8.1,4.6 \mathrm{~Hz}, 1 \mathrm{H}) .{ }^{13} \mathrm{C} \mathrm{NMR}\left(101 \mathrm{MHz}, \mathrm{CDCl}_{3}\right) \delta 158.1,148.2$, 141.4, 137.0, 133.3, 132.9, 129.1, 128.6, 127.8, 127.6, 126.9, 126.7, 126.3, 123.3, 120.1; IR (KBr), $v$ $\left(\mathrm{cm}^{-1}\right)$ 3699, 3051, 1772, 1421, 1014, 794; HRMS (ESI) m/z calcd for. $\mathrm{C}_{15} \mathrm{H}_{10} \mathrm{BrN}[\mathrm{M}+\mathrm{H}]^{+} 284.0069$, found 284.0069 .

\section{4-(3-Bromo-5-methylpyridin-2-yl)benzonitrile (12k)}

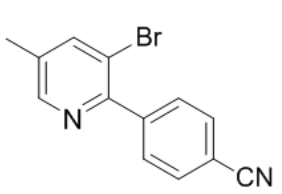

Yield: 54\% (440 mg). White solid. m.p. $139.3-139.9{ }^{\circ} \mathrm{C}$. Purified by flash column chromatography $($ EtOAc/hexane $=1 / 20) .{ }^{1} \mathrm{H}$ NMR $\left(400 \mathrm{MHz}, \mathrm{CDCl}_{3}\right) \delta 8.46(\mathrm{~s}, 1 \mathrm{H})$, 
$7.84(\mathrm{~s}, 1 \mathrm{H}), 7.79$ (d, $J=8.3 \mathrm{~Hz}, 2 \mathrm{H}), 7.73$ (d, $J=8.2 \mathrm{~Hz}, 2 \mathrm{H}), 2.39$ (s, 3H). ${ }^{13} \mathrm{C}$ NMR (101 MHz, $\left.\mathrm{CDCl}_{3}\right) \delta 153.4,149.1,143.9,142.0,134.7,131.9,130.4,119.2,118.8,112.4,17.8 . \mathrm{IR}(\mathrm{KBr}), v\left(\mathrm{~cm}^{-1}\right)$ 3689, 3055, 2223, 1791, 1452, 1020, 838; HRMS (ESI) m/z calcd for. $\mathrm{C}_{13} \mathrm{H}_{9} \mathrm{BrN}_{2}[\mathrm{M}+\mathrm{Na}]^{+} 294.9841$, found 294.9837.

\section{1-(4-(3-Bromo-5-methylpyridin-2-yl)phenyl)ethan-1-one (12l)}

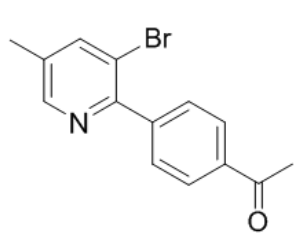

Yield: 57\% (494 mg). White solid. m.p. 89.4-90.5 ${ }^{\circ} \mathrm{C}$. Purified by flash column chromatography $($ EtOAc/hexane $=1 / 30) .{ }^{1} \mathrm{H}$ NMR $\left(400 \mathrm{MHz}, \mathrm{CDCl}_{3}\right) \delta 8.45(\mathrm{~s}$, $1 \mathrm{H}), 8.03(\mathrm{~d}, J=8.4 \mathrm{~Hz}, 2 \mathrm{H}), 7.83(\mathrm{~s}, 1 \mathrm{H}), 7.76(\mathrm{~d}, J=8.3 \mathrm{~Hz}, 2 \mathrm{H}), 2.63(\mathrm{~s}, 3 \mathrm{H})$,

$2.37(\mathrm{~s}, 3 \mathrm{H}) .{ }^{13} \mathrm{C} \mathrm{NMR}\left(101 \mathrm{MHz}, \mathrm{CDCl}_{3}\right) \delta 197.9,154.2,148.9,144.1,141.8$, 136.9, 134.2, 129.8, 128.1, 119.3, 26.8, 17.8. IR (KBr), $v\left(\mathrm{~cm}^{-1}\right)$ 3708, 2921, 1812, 1674, 1269, 842, 595; HRMS (ESI) m/z calcd for. $\mathrm{C}_{14} \mathrm{H}_{12} \mathrm{BrNO}[\mathrm{M}+\mathrm{Na}]^{+} 311.9994$, found 311.9987 .

\section{2-(Benzofuran-2-yl)-3-bromopyridine (12m)}

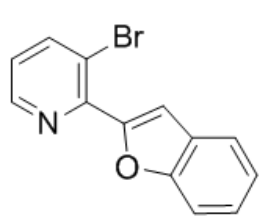

Yield: $67 \%$ (548 mg). Yellow solid. m.p. 51.0-51.8 ${ }^{\circ} \mathrm{C}$. Purified by flash column chromatography $($ EtOAc/hexane $=1 / 30) .{ }^{1} \mathrm{H}$ NMR $\left(400 \mathrm{MHz}, \mathrm{CDCl}_{3}\right) \delta 8.72(\mathrm{~d}, J=$ $4.2 \mathrm{~Hz}, 1 \mathrm{H}), 8.00(\mathrm{~d}, J=8.0 \mathrm{~Hz}, 1 \mathrm{H}), 7.84(\mathrm{~s}, 1 \mathrm{H}), 7.67$ (t, $J=8.5 \mathrm{~Hz}, 2 \mathrm{H}), 7.38$ (t, $J=$ $7.4 \mathrm{~Hz}, 1 \mathrm{H}), 7.29(\mathrm{~d}, J=7.6 \mathrm{~Hz}, 1 \mathrm{H}), 7.11(\mathrm{dd}, J=8.0,4.6 \mathrm{~Hz}, 1 \mathrm{H}) .{ }^{13} \mathrm{C}$ NMR $(101$ $\left.\mathrm{MHz}, \mathrm{CDCl}_{3}\right) \delta 154.9,152.2,148.2,147.4,142.3,128.3,126.0,123.5,123.3,122.0,118.3,112.0,110.2$. IR $(\mathrm{KBr}), v\left(\mathrm{~cm}^{-1}\right)$ 3452, 1569, 1114, 1008, 750; HRMS (ESI) m/z calcd for. $\mathrm{C}_{13} \mathrm{H}_{8} \mathrm{BrNO}[\mathrm{M}+\mathrm{Na}]^{+}$ 295.9681, found 295.9680 .

\section{3-Bromo-2-(3-methylbenzofuran-2-yl)pyridine (12n)}

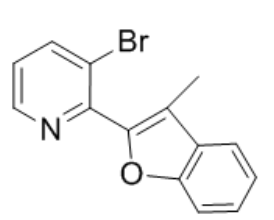

Yield: 67\% (577 mg). Colorless oil. Purified by flash column chromatography $($ EtOAc/hexane $=1 / 30) .{ }^{1} \mathrm{H}$ NMR $\left(400 \mathrm{MHz}, \mathrm{CDCl}_{3}\right) \delta 8.69(\mathrm{dd}, J=4.5,1.0 \mathrm{~Hz}, 1 \mathrm{H})$, $8.03(\mathrm{dd}, J=8.1,1.1 \mathrm{~Hz}, 1 \mathrm{H}), 7.61(\mathrm{~d}, J=7.6 \mathrm{~Hz}, 1 \mathrm{H}), 7.54(\mathrm{~d}, J=8.1 \mathrm{~Hz}, 1 \mathrm{H}), 7.36$ $(\mathrm{dd}, J=11.3,4.0 \mathrm{~Hz}, 1 \mathrm{H}), 7.29(\mathrm{t}, J=7.3 \mathrm{~Hz}, 1 \mathrm{H}), 7.20(\mathrm{dd}, J=8.1,4.6 \mathrm{~Hz}, 1 \mathrm{H})$, 2.39 (s, 3H). ${ }^{13} \mathrm{C}$ NMR $\left(101 \mathrm{MHz}, \mathrm{CDCl}_{3}\right) \delta 154.4,149.8,148.8,148.1,141.6,130.0,125.3,124.3$, 122.7, 121.1, 120.1, 116.4, 111.6, 9.5. IR (KBr), $v\left(\mathrm{~cm}^{-1}\right)$ 3685, 2921, 1728, 1440, 1267, 1010, 746; HRMS (ESI) m/z calcd for. $\mathrm{C}_{14} \mathrm{H}_{10} \mathrm{BrNO}[\mathrm{M}+\mathrm{H}]^{+} 288.0019$, found 288.0018.

\section{3-Bromo-4-phenylpyridine (13a)}<smiles>Brc1cnccc1-c1ccccc1</smiles>

Yield: 65\% (460mg). Colorless oil. Purified by flash column chromatography (EtOAc/ hexane $=1 / 30) .{ }^{1} \mathrm{H}$ NMR $\left(400 \mathrm{MHz}, \mathrm{CDCl}_{3}\right) \delta 8.81(\mathrm{~s}, 1 \mathrm{H}), 8.52(\mathrm{~d}, J=5.0 \mathrm{~Hz}, 1 \mathrm{H})$, $7.44(\mathrm{dd}, J=4.1,2.6 \mathrm{~Hz}, 5 \mathrm{H}), 7.24(\mathrm{~d}, J=4.9 \mathrm{~Hz}, 1 \mathrm{H}){ }^{[4]}$

\section{3-Bromo-4-(p-tolyl)pyridine (13b)}

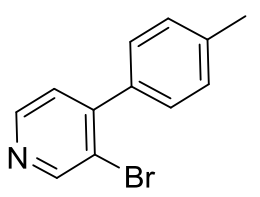

Yield: $57 \%$ (403mg). Colorless oil. Purified by flash column chromatography $($ EtOAc/hexane $=1 / 30) .{ }^{1} \mathrm{H}$ NMR $\left(400 \mathrm{MHz}, \mathrm{CDCl}_{3}\right) \delta 8.81(\mathrm{~s}, 1 \mathrm{H}), 8.52(\mathrm{~d}, J=5.1$ $\mathrm{Hz}, 1 \mathrm{H}), 7.37-7.33$ (m, 2H), $7.30-7.27$ (m, 2H), 7.25 (d, J = 4.9 Hz, 1H), 2.43 (s, 
$3 \mathrm{H}){ }^{[3]}$

1-(4-(3-Bromopyridin-4-yl)phenyl)ethan-1-one (13c)

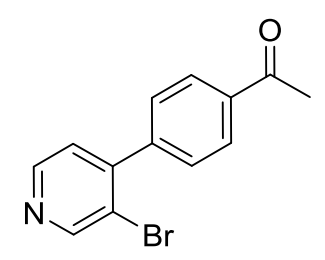

Yield: $70 \%$ (495mg). Yellow solid. m.p. $76.0-77.2{ }^{\circ} \mathrm{C}$. Purified by flash column chromatography $($ EtOAc/hexane $=1 / 30) .{ }^{1} \mathrm{H}$ NMR $\left(400 \mathrm{MHz}, \mathrm{CDCl}_{3}\right) \delta 8.84(\mathrm{~s}$, $1 \mathrm{H}), 8.58(\mathrm{~d}, J=5.0 \mathrm{~Hz}, 1 \mathrm{H}), 8.06(\mathrm{~d}, J=8.2 \mathrm{~Hz}, 2 \mathrm{H}), 7.53(\mathrm{~d}, J=8.4 \mathrm{~Hz}, 2 \mathrm{H})$,

$7.27(\mathrm{~d}, J=4.9 \mathrm{~Hz}, 1 \mathrm{H}), 2.66(\mathrm{~s}, 3 \mathrm{H}) .{ }^{13} \mathrm{C} \mathrm{NMR}\left(101 \mathrm{MHz}, \mathrm{CDCl}_{3}\right) \delta 197.6,152.8$, 148.8, 148.6, 142.8, 137.3, 129.3, 129.2, 128.5, 127.6, 125.4, 120.6, 26.8; IR (KBr), v (cm $\left.{ }^{-1}\right) 3446$, 2921, 1938, 1674, 1269, 821, 595; HRMS (ESI) m/z calcd for. $\mathrm{C}_{13} \mathrm{H}_{10} \mathrm{BrNO}[\mathrm{M}+\mathrm{Na}]^{+} 297.9838$, found 297.9829.

\section{3-Bromo-4-(furan-2-yl)pyridine (14a)}

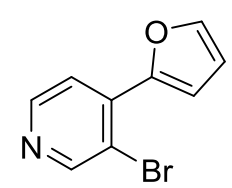

Yield: 44\% (311mg). Colorless oil. Purified by flash column chromatography (EtOAc/ hexane = 1/30). ${ }^{1} \mathrm{H} \mathrm{NMR}\left(400 \mathrm{MHz}, \mathrm{CDCl}_{3}\right) \delta 8.74(\mathrm{~s}, 1 \mathrm{H}), 8.47(\mathrm{~d}, J=5.3 \mathrm{~Hz}, 1 \mathrm{H})$, $7.67(\mathrm{~d}, J=5.3 \mathrm{~Hz}, 1 \mathrm{H}), 7.55(\mathrm{~d}, J=1.8 \mathrm{~Hz}, 1 \mathrm{H}), 7.48(\mathrm{~d}, J=3.6 \mathrm{~Hz}, 1 \mathrm{H}), 6.64-6.43$ $(\mathrm{m}, 1 \mathrm{H}) ;{ }^{13} \mathrm{C}$ NMR $\left(101 \mathrm{MHz}, \mathrm{CDCl}_{3}\right) \delta 153.3,148.6,148.0,143.7,136.9,121.1,116.1,113.9,112.1$. IR $(\mathrm{KBr}), v\left(\mathrm{~cm}^{-1}\right) 3450,2870,1456,998,786$; HRMS (ESI) m/z calcd for. $\mathrm{C}_{9} \mathrm{H}_{6} \mathrm{BrNO}[\mathrm{M}+\mathrm{H}]^{+}$ 223.9706, found 223.9710.

\section{3-Bromo-4-(5-methylfuran-2-yl)pyridine (14b)}

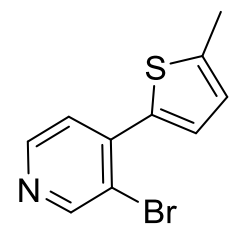

Yield: $10 \%$ (71mg). Yellow oil. Purified by flash column chromatography (EtOAc/ hexane = 1/30). ${ }^{1} \mathrm{H} \mathrm{NMR}\left(400 \mathrm{MHz}, \mathrm{CDCl}_{3}\right) \delta 8.77(\mathrm{~s}, 1 \mathrm{H}), 8.44(\mathrm{~d}, J=5.1 \mathrm{~Hz}, 1 \mathrm{H})$, $7.43(\mathrm{~d}, J=3.7 \mathrm{~Hz}, 1 \mathrm{H}), 7.38(\mathrm{~d}, J=5.1 \mathrm{~Hz}, 1 \mathrm{H}), 6.82(\mathrm{~d}, J=3.6 \mathrm{~Hz}, 1 \mathrm{H}), 2.54(\mathrm{~s}, 3 \mathrm{H})$.

${ }^{13} \mathrm{C}$ NMR $\left(101 \mathrm{MHz}, \mathrm{CDCl}_{3}\right) \delta 153.5,148.2,143.5,142.2,136.1,129.5,126.1,124.7$, 119.2, 15.5. IR (KBr), $v\left(\mathrm{~cm}^{-1}\right) 3444,2920,1577,1024,802$; HRMS (ESI) m/z calcd for. $\mathrm{C}_{10} \mathrm{H}_{8} \mathrm{BrNS}$ $[\mathrm{M}+\mathrm{H}]^{+} 253.9634$, found 253.9630 .

\section{1-(5-(3-Bromopyridin-4-yl)furan-2-yl)ethan-1-one (14c)}

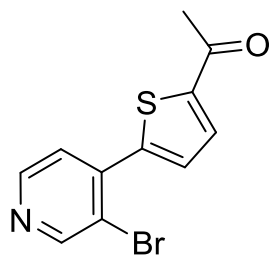

Yield: 45\% (318mg). White solid. m.p. 87.3-88.7 ${ }^{\circ} \mathrm{C}$. Purified by flash column chromatography $($ EtOAc/hexane $=1 / 30) .{ }^{1} \mathrm{H}$ NMR $\left(400 \mathrm{MHz}, \mathrm{CDCl}_{3}\right) \delta 8.84(\mathrm{~s}, 1 \mathrm{H})$, $8.54(\mathrm{~d}, J=5.1 \mathrm{~Hz}, 1 \mathrm{H}), 7.70(\mathrm{~d}, J=4.0 \mathrm{~Hz}, 1 \mathrm{H}), 7.52(\mathrm{~d}, J=4.0 \mathrm{~Hz}, 1 \mathrm{H}), 7.42(\mathrm{~d}, J$ $=4.9 \mathrm{~Hz}, 1 \mathrm{H}), 2.59(\mathrm{~s}, 3 \mathrm{H}) .{ }^{13} \mathrm{C} \mathrm{NMR}\left(101 \mathrm{MHz}, \mathrm{CDCl}_{3}\right) \delta 190.7,153.6,148.6,146.3$, 146.0, 141.3, 132.2, 129.8, 125.1, 119.9, 26.9. IR (KBr), $v\left(\mathrm{~cm}^{-1}\right) 3296,2921,1799,1664,1278,1247$, 923, 808, 594; HRMS (ESI) m/z calcd for. $\mathrm{C}_{11} \mathrm{H}_{8} \mathrm{BrNSO}[\mathrm{M}+\mathrm{Na}]^{+}$303.9402, found 303.9401.

\section{4-(Benzofuran-2-yl)-3-bromopyridine (14d)}

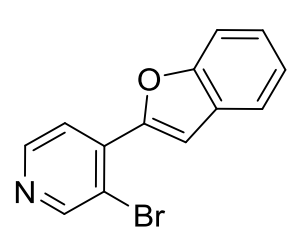

Yield: $40 \%$ (283mg). White solid. m.p. 96.6-97.2 ${ }^{\circ} \mathrm{C}$. Purified by flash column chromatography $($ EtOAc/hexane $=1 / 30) .{ }^{1} \mathrm{H}$ NMR $\left(400 \mathrm{MHz}, \mathrm{CDCl}_{3}\right) \delta 8.80(\mathrm{~s}, 1 \mathrm{H})$, $8.55(\mathrm{~d}, J=5.2 \mathrm{~Hz}, 1 \mathrm{H}), 7.89-7.83(\mathrm{~m}, 2 \mathrm{H}), 7.67-7.62(\mathrm{~m}, 1 \mathrm{H}), 7.54-7.50(\mathrm{~m}$, $1 \mathrm{H}), 7.40-7.34(\mathrm{~m}, 1 \mathrm{H}), 7.30-7.24(\mathrm{~m}, 1 \mathrm{H}) .{ }^{13} \mathrm{C} \mathrm{NMR}\left(101 \mathrm{MHz}, \mathrm{CDCl}_{3}\right) \delta 154.5$, 
153.6, 150.2, 148.4, 137.2, 128.3, 126.4, 123.5, 122.3, 122.2, 117.5, 111.4, 110.3. IR (KBr), $v\left(\mathrm{~cm}^{-1}\right)$ 3448, 2923, 1787, 1579, 1161, 1012, 750; HRMS (ESI) m/z calcd for. $\mathrm{C}_{13} \mathrm{H}_{8} \mathrm{BrNO}[\mathrm{M}+\mathrm{H}]^{+} 273.9862$, found 273.9865 .

The data of 1a-1t, 3a-3d, 4a-4i, 7a-7d, 9a.

3-(Furan-2-yl)-2-phenylpyridine (1a)

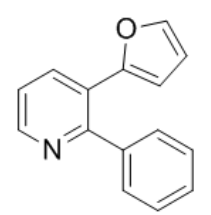

Yield: $60 \%$ (132 mg). White solid. m.p. 59.5-60.4 ${ }^{\circ} \mathrm{C}$. Purified by flash column chromatography $($ EtOAc/hexane $=1 / 40) .{ }^{1} \mathrm{H} \mathrm{NMR}\left(400 \mathrm{MHz}, \mathrm{CDCl}_{3}\right) \delta 8.64-8.55(\mathrm{~m}$, 1H), $8.12-8.06(\mathrm{~m}, 1 \mathrm{H}), 7.48-7.38(\mathrm{~m}, 6 \mathrm{H}), 7.32$ (dd, $J=7.9,4.8 \mathrm{~Hz}, 1 \mathrm{H}), 6.28$ (dd, $J$ $=3.1,1.6 \mathrm{~Hz}, 1 \mathrm{H}), 5.79(\mathrm{~d}, J=3.3 \mathrm{~Hz}, 1 \mathrm{H}) .{ }^{13} \mathrm{C} \mathrm{NMR}\left(101 \mathrm{MHz}, \mathrm{CDCl}_{3}\right) \delta 156.4,151.0$, 148.0, 142.5, 141.0, 135.0, 128.8, 128.5, 128.4, 125.6, 122.3, 111.7, 110.0. IR (KBr), $v\left(\mathrm{~cm}^{-1}\right) 3708$, 3114, 1745, 1434, 1149, 999, 754, 594; HRMS (ESI) m/z calcd for. $\mathrm{C}_{15} \mathrm{H}_{11} \mathrm{NO}[\mathrm{M}+\mathrm{H}]^{+} 222.0913$, found 222.0914.

\section{2-Phenyl-3-(thiophen-2-yl)pyridine (1b)}

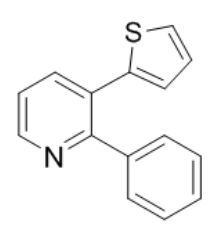

Yield: $58 \%$ (137 mg). White solid. m.p. 44.5-46.7 ${ }^{\circ} \mathrm{C}$. Purified by flash column chromatography $($ EtOAc/hexane $=1 / 40) .{ }^{1} \mathrm{H} \mathrm{NMR}\left(400 \mathrm{MHz}, \mathrm{CDCl}_{3}\right) \delta 8.70-8.61(\mathrm{~m}$, $1 \mathrm{H}), 7.88-7.76(\mathrm{~m}, 1 \mathrm{H}), 7.47-7.40(\mathrm{~m}, 2 \mathrm{H}), 7.36-7.27(\mathrm{~m}, 4 \mathrm{H}), 7.26-7.24(\mathrm{~m}, 1 \mathrm{H})$, $6.95-6.87(\mathrm{~m}, 1 \mathrm{H}), 6.79(\mathrm{~d}, J=3.3 \mathrm{~Hz}, 1 \mathrm{H}) .{ }^{13} \mathrm{C} \mathrm{NMR}\left(101 \mathrm{MHz}, \mathrm{CDCl}_{3}\right) \delta 157.7$, 148.6, 141.3, 140.3, 138.6, 129.6, 129.3, 128.2, 128.2, 127.6, 127.4, 126.5, 122.2. IR (KBr), $v\left(\mathrm{~cm}^{-1}\right)$ $3678,3055,2455,1953,1431,1226,1016,702$, 619; HRMS (ESI) m/z calcd for. $\mathrm{C}_{15} \mathrm{H}_{11} \mathrm{NS}[\mathrm{M}+\mathrm{H}]^{+}$ 238.0685, found 238.0678 .

\section{3-(5-Methylthiophen-2-yl)-2-phenylpyridine (1c)}

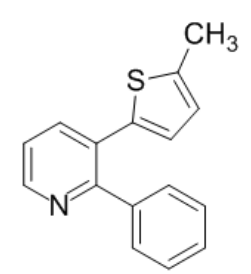

Yield: 59\% (148 mg). Yellow oil. Purified by flash column chromatography (EtOAc/ hexane $=1 / 40) .{ }^{1} \mathrm{H} \mathrm{NMR}\left(400 \mathrm{MHz}, \mathrm{CDCl}_{3}\right) \delta 8.59(\mathrm{dd}, J=4.7,1.4 \mathrm{~Hz}, 1 \mathrm{H}), 7.76(\mathrm{dd}$, $J=7.8,1.4 \mathrm{~Hz}, 1 \mathrm{H}), 7.49-7.42(\mathrm{~m}, 2 \mathrm{H}), 7.34-7.29(\mathrm{~m}, 3 \mathrm{H}), 7.23(\mathrm{dd}, J=7.8,4.8$

$\mathrm{Hz}, 1 \mathrm{H}), 6.54$ (s, 2H), 2.40 (s, 3H). ${ }^{13} \mathrm{C} \mathrm{NMR}\left(101 \mathrm{MHz}, \mathrm{CDCl}_{3}\right) \delta 157.3,148.1,141.1$, $140.4,138.7,138.2,129.5,128.1,127.5,125.7,122.1,15.3 . \mathrm{IR}(\mathrm{KBr}), v\left(\mathrm{~cm}^{-1}\right) 3699,3055,1739,1425$, 1018, 796; HRMS (ESI) m/z calcd for. $\mathrm{C}_{16} \mathrm{H}_{13} \mathrm{NS}[\mathrm{M}+\mathrm{H}]^{+} 252.0841$, found 252.0838.

\section{3-(Furan-2-yl)-2-(p-tolyl)pyridine (1d)}

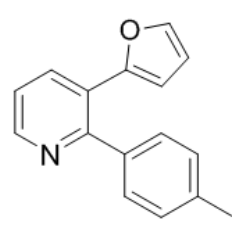

Yield: 65\% (153 mg). Yellow oil. Purified by flash column chromatography (EtOAc/ hexane $=1 / 40) .{ }^{1} \mathrm{H}$ NMR $\left(600 \mathrm{MHz}, \mathrm{CDCl}_{3}\right) \delta 8.58(\mathrm{dd}, J=4.7,1.7 \mathrm{~Hz}, 1 \mathrm{H}), 8.05(\mathrm{dd}$, $J=7.9,1.7 \mathrm{~Hz}, 1 \mathrm{H}), 7.39(\mathrm{~d}, J=1.2 \mathrm{~Hz}, 1 \mathrm{H}), 7.34(\mathrm{~d}, J=8.1 \mathrm{~Hz}, 2 \mathrm{H}), 7.29$ (dd, $J=7.9$, $4.7 \mathrm{~Hz}, 1 \mathrm{H}), 7.21(\mathrm{~d}, J=7.8 \mathrm{~Hz}, 2 \mathrm{H}), 6.29(\mathrm{dd}, J=3.4,1.8 \mathrm{~Hz}, 1 \mathrm{H}), 5.89-5.78(\mathrm{~m}$, 
1H), $2.40(\mathrm{~s}, 3 \mathrm{H}) .{ }^{13} \mathrm{C} \mathrm{NMR}\left(151 \mathrm{MHz}, \mathrm{CDCl}_{3}\right) \delta 156.5,151.2,148.0,142.4,138.2,138.1,135.1,129.2$, 128.7, 125.6, 122.0, 111.6, 109.9, 21.5. IR (KBr), $v\left(\mathrm{~cm}^{-1}\right)$ 3427, 3051, 1434, 1269, 1008, 744; HRMS (ESI) $\mathrm{m} / \mathrm{z}$ calcd for. $\mathrm{C}_{16} \mathrm{H}_{13} \mathrm{NO}[\mathrm{M}+\mathrm{H}]^{+} 236.1070$, found 236.1067 .

\section{3-(Furan-2-yl)-2-(4-methoxyphenyl)pyridine (1e)}

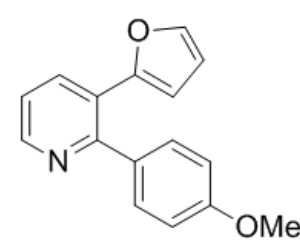

Yield: 68\% (170 mg). White solid. m.p. 86.6-87.7 ${ }^{\circ} \mathrm{C}$. Purified by flash column chromatography $($ EtOAc/hexane $=1 / 40) .{ }^{1} \mathrm{H}$ NMR $\left(400 \mathrm{MHz}, \mathrm{CDCl}_{3}\right) \delta 8.56(\mathrm{dd}, J$ $=4.7,1.7 \mathrm{~Hz}, 1 \mathrm{H}), 8.02(\mathrm{dd}, J=7.9,1.7 \mathrm{~Hz}, 1 \mathrm{H}), 7.46-7.32(\mathrm{~m}, 3 \mathrm{H}), 7.26(\mathrm{dd}, J=$ $7.9,4.8 \mathrm{~Hz}, 1 \mathrm{H}), 6.95-6.90(\mathrm{~m}, 2 \mathrm{H}), 6.30(\mathrm{dd}, J=3.4,1.8 \mathrm{~Hz}, 1 \mathrm{H}), 5.89(\mathrm{~d}, J=$

$3.3 \mathrm{~Hz}, 1 \mathrm{H}), 3.83(\mathrm{~s}, 3 \mathrm{H}) .{ }^{13} \mathrm{C}$ NMR $\left(101 \mathrm{MHz}, \mathrm{CDCl}_{3}\right) \delta 159.8,156.1,151.3,148.0,142.4,135.2$, 133.4, 130.2, 125.5, 121.8, 113.9, 111.6, 109.8, 55.3. IR (KBr), $v\left(\mathrm{~cm}^{-1}\right)$ 2908, 2842, 2532, 1897, 1434 , 1245, 1018, 740, 588; HRMS (ESI) m/z calcd for. $\mathrm{C}_{16} \mathrm{H}_{13} \mathrm{NO}_{2}[\mathrm{M}+\mathrm{H}]^{+} 252.1019$, found 252.1016.

\section{2-(4-Fluorophenyl)-3-(furan-2-yl)pyridine (1f)}

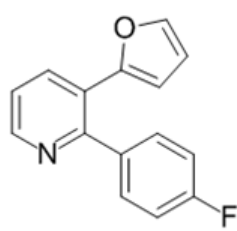

Yield: $63 \%(150 \mathrm{mg})$. Yellow oil. Purified by flash column chromatography (EtOAc/ hexane $=1 / 40) .{ }^{1} \mathrm{H}$ NMR $\left(400 \mathrm{MHz}, \mathrm{CDCl}_{3}\right) \delta 8.58(\mathrm{dd}, J=4.6,1.1 \mathrm{~Hz}, 1 \mathrm{H}), 8.05(\mathrm{dd}$, $J=7.8,1.3 \mathrm{~Hz}, 1 \mathrm{H}), 7.47-7.38(\mathrm{~m}, 3 \mathrm{H}), 7.32(\mathrm{dd}, J=7.8,4.8 \mathrm{~Hz}, 1 \mathrm{H}), 7.10(\mathrm{dd}, J=$

14.2, $5.6 \mathrm{~Hz}, 2 \mathrm{H}), 6.32(\mathrm{dd}, J=3.2,1.6 \mathrm{~Hz}, 1 \mathrm{H}), 5.87(\mathrm{~d}, J=3.3 \mathrm{~Hz}, 1 \mathrm{H}) .{ }^{13} \mathrm{C} \mathrm{NMR}$ $\left(101 \mathrm{MHz}, \mathrm{CDCl}_{3}\right) \delta 163.0\left(\mathrm{~d},{ }^{1} \mathrm{~J}=246.0 \mathrm{~Hz}\right), 155.3,151.0,148.2,142.6,137.0,135.5,130.8\left(\mathrm{~d},{ }^{3} \mathrm{~J}=\right.$ 8.4 Hz), 125.7, 122.4, $115.5\left(\mathrm{~d},{ }^{2} J=21 \mathrm{~Hz}\right), 111.7,110.0$. IR $(\mathrm{KBr}), v\left(\mathrm{~cm}^{-1}\right) 3747,3053,1720,1436$, 1224, 842, 779, 572; HRMS (ESI) m/z calcd for. $\mathrm{C}_{15} \mathrm{H}_{10} \mathrm{FNO}[\mathrm{M}+\mathrm{H}]^{+} 240.0819$, found 240.0817 .

\section{1-(4-(3-(Furan-2-yl)pyridin-2-yl)phenyl)ethan-1-one (1g)}

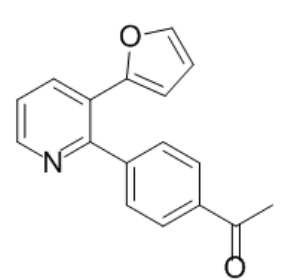

Yield: $67 \%$ (176 mg). White solid. m.p. 105.0-105.6 ${ }^{\circ} \mathrm{C}$. Purified by flash column chromatography $($ EtOAc/hexane $=1 / 40) .{ }^{1} \mathrm{H}$ NMR $\left(400 \mathrm{MHz}, \mathrm{CDCl}_{3}\right) \delta 8.60(\mathrm{dd}, J$ = 4.7, $1.6 \mathrm{~Hz}, 1 \mathrm{H}), 8.04(\mathrm{dd}, J=7.9,1.6 \mathrm{~Hz}, 1 \mathrm{H}), 8.00-7.96(\mathrm{~m}, 2 \mathrm{H}), 7.57-7.52$ $(\mathrm{m}, 2 \mathrm{H}), 7.39-7.30(\mathrm{~m}, 2 \mathrm{H}), 6.30(\mathrm{dd}, J=3.3,1.8 \mathrm{~Hz}, 1 \mathrm{H}), 5.90(\mathrm{~d}, J=3.4 \mathrm{~Hz}, 1 \mathrm{H})$, 2.62 (s, 3H). ${ }^{13} \mathrm{C} \mathrm{NMR}\left(101 \mathrm{MHz}, \mathrm{CDCl}_{3}\right) \delta 198.0,155.1,150.7,148.3,145.6,142.8$, 136.7, 135.6, 129.3, 128.5, 125.7, 122.8, 111.7, 110.2, 26.8. IR (KBr), v (cm $\left.{ }^{-1}\right) 3448,3141,2918,1741$, 1677, 1267, 1004, 813, 752; HRMS (ESI) m/z calcd for. $\mathrm{C}_{17} \mathrm{H}_{13} \mathrm{NO}_{2}[\mathrm{M}+\mathrm{Na}]^{+} 286.0838$, found 286.0834 .

\section{4-(3-(Furan-2-yl)pyridin-2-yl)benzonitrile (1h)}

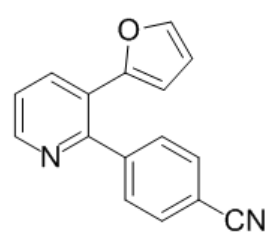

Yield: $61 \%(150 \mathrm{mg})$. Yellow solid. m.p. 87.5-88.0 ${ }^{\circ} \mathrm{C}$. Purified by flash column chromatography $($ EtOAc/hexane $=1 / 30) .{ }^{1} \mathrm{H}$ NMR $\left(400 \mathrm{MHz}, \mathrm{CDCl}_{3}\right) \delta 8.61(\mathrm{dd}, J$ $=4.7,1.5 \mathrm{~Hz}, 1 \mathrm{H}), 8.03(\mathrm{dd}, J=7.7,1.2 \mathrm{~Hz}, 1 \mathrm{H}), 7.67(\mathrm{~d}, J=8.2 \mathrm{~Hz}, 2 \mathrm{H}), 7.55(\mathrm{~d}, J$ $=8.2 \mathrm{~Hz}, 2 \mathrm{H}), 7.36(\mathrm{dd}, J=8.0,4.4 \mathrm{~Hz}, 2 \mathrm{H}), 6.34(\mathrm{dd}, J=3.2,1.6 \mathrm{~Hz}, 1 \mathrm{H}), 5.99(\mathrm{~d}$, 
$J=3.3 \mathrm{~Hz}, 1 \mathrm{H}) .{ }^{13} \mathrm{C} \mathrm{NMR}\left(101 \mathrm{MHz}, \mathrm{CDCl}_{3}\right) \delta 154.1,150.5,148.5,145.4,143.0,136.0,132.2,129.8$, 125.7, 123.1, 118.9, 112.0, 111.8, 110.2. IR (KBr), $v\left(\mathrm{~cm}^{-1}\right)$ 3685, 3053, 2225, 1728, 1434, 1274, 840, 736, 570; HRMS (ESI) m/z calcd for. $\mathrm{C}_{16} \mathrm{H}_{10} \mathrm{~N}_{2} \mathrm{O}[\mathrm{M}+\mathrm{H}]^{+} 247.0866$, found 247.0868.

\section{4-(3-(Benzofuran-2-yl)pyridin-2-yl)benzonitrile (1i)}

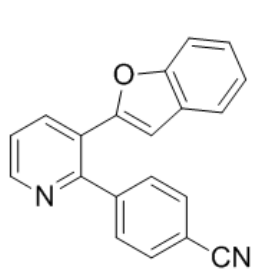

Yield: 64\% (189 mg). White solid. m.p. 133.1-134.4 ${ }^{\circ} \mathrm{C}$. Purified by flash column chromatography $($ EtOAc/hexane $=1 / 30) .{ }^{1} \mathrm{H}$ NMR $\left(400 \mathrm{MHz}, \mathrm{CDCl}_{3}\right) \delta 8.69(\mathrm{~d}, J=$ $4.7 \mathrm{~Hz}, 1 \mathrm{H}), 8.22(\mathrm{~d}, J=7.9 \mathrm{~Hz}, 1 \mathrm{H}), 7.66(\mathrm{dd}, J=21.6,8.1 \mathrm{~Hz}, 4 \mathrm{H}), 7.44(\mathrm{ddd}, J=$ $18.9,14.4,8.0 \mathrm{~Hz}, 3 \mathrm{H}), 7.29$ (t, $J=7.7 \mathrm{~Hz}, 1 \mathrm{H}), 7.22$ (t, $J=7.4 \mathrm{~Hz}, 1 \mathrm{H}), 6.34(\mathrm{~s}, 1 \mathrm{H})$.

${ }^{13} \mathrm{C}$ NMR $\left(101 \mathrm{MHz}, \mathrm{CDCl}_{3}\right) \delta 155.1,154.8,152.5,149.3,145.1,136.8,132.3,129.9$, 128.5, 125.6, 125.2, 123.4, 123.1, 121.4, 118.8, 112.3, 111.3, 106.8. IR (KBr), v (cm $\left.{ }^{-1}\right) 3697,2923$, 2229, 1728, 1434, 1010, 746; HRMS (ESI) m/z calcd for. $\mathrm{C}_{20} \mathrm{H}_{12} \mathrm{~N}_{2} \mathrm{O}[\mathrm{M}+\mathrm{H}]^{+} 297.1022$, found 297.1020 .

\section{4-(3-(5-Methylthiophen-2-yl)pyridin-2-yl)benzonitrile (1j)}

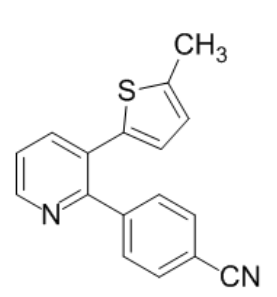

Yield: $67 \%$ (184 mg). Yellow solid. m.p. $100.5-102.5{ }^{\circ} \mathrm{C}$. Purified by flash column chromatography $($ EtOAc/hexane $=1 / 30) .{ }^{1} \mathrm{H}$ NMR $\left(400 \mathrm{MHz}, \mathrm{CDCl}_{3}\right) \delta 8.60(\mathrm{~d}, J=$ $3.8 \mathrm{~Hz}, 1 \mathrm{H}), 7.80-7.75(\mathrm{~m}, 1 \mathrm{H}), 7.61-7.54(\mathrm{~m}, 4 \mathrm{H}), 7.30(\mathrm{dd}, J=7.8,4.8 \mathrm{~Hz}, 1 \mathrm{H})$, $6.57(\mathrm{~d}, J=2.7 \mathrm{~Hz}, 1 \mathrm{H}), 6.52(\mathrm{~d}, J=3.4 \mathrm{~Hz}, 1 \mathrm{H}), 2.41(\mathrm{~s}, 3 \mathrm{H}) .{ }^{13} \mathrm{C}$ NMR $(101 \mathrm{MHz}$, $\left.\mathrm{CDCl}_{3}\right) \delta 155.0,148.4,144.8,141.8,138.6,137.6,131.8,130.3,129.7,127.8,125.9$, 122.9, 118.8, 111.7, 15.3. IR (KBr), $v\left(\mathrm{~cm}^{-1}\right)$ 3691, 2916, 2223, 1728, 1425, 1269, 804, 572; HRMS (ESI) $\mathrm{m} / \mathrm{z}$ calcd for. $\mathrm{C}_{17} \mathrm{H}_{12} \mathrm{~N}_{2} \mathrm{~S}[\mathrm{M}+\mathrm{Na}]^{+} 299.0613$, found 299.0615 .

\section{3-(Furan-2-yl)-2-(4-(trifluoromethyl)phenyl)pyridine (1k)}

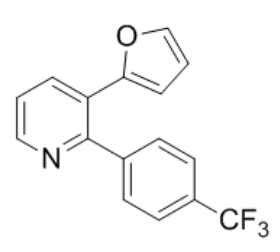

Yield: 67\% (193 mg). Yellow oil. Purified by flash column chromatography (EtOAc/ hexane $=1 / 40) .{ }^{1} \mathrm{H}$ NMR $\left(400 \mathrm{MHz}, \mathrm{CDCl}_{3}\right) \delta 8.61(\mathrm{dd}, J=4.7,1.6 \mathrm{~Hz}, 1 \mathrm{H}), 8.06$ $(\mathrm{dd}, J=8.0,1.6 \mathrm{~Hz}, 1 \mathrm{H}), 7.66(\mathrm{~d}, J=8.1 \mathrm{~Hz}, 2 \mathrm{H}), 7.58(\mathrm{~d}, J=8.1 \mathrm{~Hz}, 2 \mathrm{H}), 7.43-$

$7.31(\mathrm{~m}, 2 \mathrm{H}), 6.33(\mathrm{dd}, J=3.4,1.8 \mathrm{~Hz}, 1 \mathrm{H}), 5.92(\mathrm{~d}, J=3.4 \mathrm{~Hz}, 1 \mathrm{H}) .{ }^{13} \mathrm{C}$ NMR $(101$ $\left.\mathrm{MHz}, \mathrm{CDCl}_{3}\right) \delta 154.8,150.7,148.4,144.5,142.9,141.6,135.7,130.4\left(\mathrm{q},{ }^{2} J=32 \mathrm{~Hz}\right), 129.4,125.8$, 125.4(q, $\left.{ }^{3} J=3.7 \mathrm{~Hz}\right), 125.1,124.4\left(\mathrm{q},{ }^{1} J=270 \mathrm{~Hz}\right), 124.0,122.8,111.8,110.2$. IR $(\mathrm{KBr}), v\left(\mathrm{~cm}^{-1}\right) 3417$, 3053, 1568, 1326, 1126, 848; HRMS (ESI) m/z calcd for. $\mathrm{C}_{16} \mathrm{H}_{10} \mathrm{~F}_{3} \mathrm{NO}[\mathrm{M}+\mathrm{H}]^{+} 290.0787$, found 290.0791 .

\section{3-(5-Methylthiophen-2-yl)-2-(4-(trifluoromethyl)phenyl)pyridine (11)}

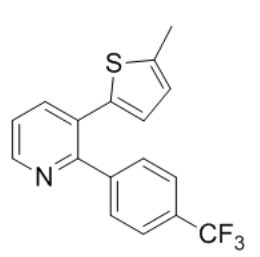

Yield: 69\% (220 mg). Yellow oil. Purified by flash column chromatography (EtOAc/ hexane $=1 / 40) .{ }^{1} \mathrm{H}$ NMR $\left(400 \mathrm{MHz}, \mathrm{CDCl}_{3}\right) \delta 8.63(\mathrm{dd}, J=4.7,1.5 \mathrm{~Hz}, 1 \mathrm{H}), 7.81(\mathrm{dd}$, $J=7.8,1.5 \mathrm{~Hz}, 1 \mathrm{H}), 7.63-7.57(\mathrm{~m}, 4 \mathrm{H}), 7.31(\mathrm{dd}, J=7.8,4.8 \mathrm{~Hz}, 1 \mathrm{H}), 6.61-6.50(\mathrm{~m}$,

2H), $2.44(\mathrm{~s}, 3 \mathrm{H}) .{ }^{13} \mathrm{C}$ NMR $\left(101 \mathrm{MHz}, \mathrm{CDCl}_{3}\right) \delta 155.8,148.5,144.1,141.7,139.8$, 138.6, 138.0, 130.2(q, $\left.{ }^{2} J=30 \mathrm{~Hz}\right), 130.0,129.8,129.7,127.9,126.0,125.1\left(\mathrm{q},{ }^{3} J=4 \mathrm{~Hz}\right), 124.3\left(\mathrm{q},{ }^{1} J=\right.$ 
$270 \mathrm{~Hz}), 122.7,120.2$, 15.4. IR (KBr), $v\left(\mathrm{~cm}^{-1}\right)$ 3689, 3051, 1739, 1429, 1325, 1126, 798; HRMS (ESI) $\mathrm{m} / \mathrm{z}$ calcd for. $\mathrm{C}_{17} \mathrm{H}_{12} \mathrm{~F}_{3} \mathrm{NS}[\mathrm{M}+\mathrm{H}]^{+} 320.0715$, found 320.0709 .

\section{3-(3-(Furan-2-yl)pyridin-2-yl)benzonitrile (1m)}

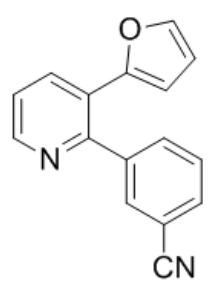

Yield: $71 \%$ (174 mg). White solid. m.p. 64.3-65.9 ${ }^{\circ} \mathrm{C}$. Purified by flash column chromatography $($ EtOAc/hexane $=1 / 30) .{ }^{1} \mathrm{H} \mathrm{NMR}\left(400 \mathrm{MHz}, \mathrm{CDCl}_{3}\right) \delta 8.62(\mathrm{dd}, J=$ 4.7, $1.4 \mathrm{~Hz}, 1 \mathrm{H}), 8.04(\mathrm{dd}, J=7.9,1.4 \mathrm{~Hz}, 1 \mathrm{H}), 7.77(\mathrm{~s}, 1 \mathrm{H}), 7.71-7.65(\mathrm{~m}, 2 \mathrm{H}), 7.50$ (t, $J=7.8 \mathrm{~Hz}, 1 \mathrm{H}), 7.38(\mathrm{dd}, J=8.3,3.3 \mathrm{~Hz}, 2 \mathrm{H}), 6.36(\mathrm{dd}, J=3.3,1.7 \mathrm{~Hz}, 1 \mathrm{H}), 5.98(\mathrm{~d}$, $J=3.4 \mathrm{~Hz}, 1 \mathrm{H}) .{ }^{13} \mathrm{C} \mathrm{NMR}\left(101 \mathrm{MHz}, \mathrm{CDCl}_{3}\right) \delta 153.8,150.5,148.6,143.1,142.1$, 136.0, 133.5, 132.8, 131.9, 129.2, 125.8, 123.1, 118.7, 112.7, 111.9, 110.3. IR (KBr), $v\left(\mathrm{~cm}^{-1}\right) 3685$, 3114, 2921, 2225, 1731, 1440, 1164, 1002, 748; HRMS (ESI) m/z calcd for. $\mathrm{C}_{16} \mathrm{H}_{10} \mathrm{~N}_{2} \mathrm{O}[\mathrm{M}+\mathrm{H}]^{+}$ 247.0866, found 247.0863.

\section{1-(3-(3-(Furan-2-yl)pyridin-2-yl)phenyl)ethan-1-one (1n)}

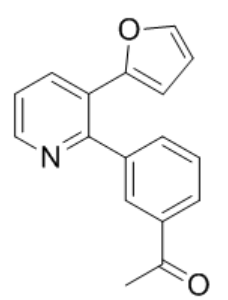

Yield: 73\% (192 mg). Yellow oil. Purified by flash column chromatography (EtOAc/ hexane $=1 / 40) .{ }^{1} \mathrm{H}$ NMR $\left(400 \mathrm{MHz}, \mathrm{CDCl}_{3}\right) \delta 8.62(\mathrm{~d}, J=4.5 \mathrm{~Hz}, 1 \mathrm{H}), 8.10-7.98(\mathrm{~m}$, $3 \mathrm{H}), 7.66(\mathrm{~d}, J=7.6 \mathrm{~Hz}, 1 \mathrm{H}), 7.50(\mathrm{t}, J=7.7 \mathrm{~Hz}, 1 \mathrm{H}), 7.36(\mathrm{dd}, J=8.8,5.6 \mathrm{~Hz}, 2 \mathrm{H})$, $6.31(\mathrm{~d}, J=3.2 \mathrm{~Hz}, 1 \mathrm{H}), 5.90(\mathrm{~d}, J=3.4 \mathrm{~Hz}, 1 \mathrm{H}), 2.57(\mathrm{~s}, 3 \mathrm{H}) .{ }^{13} \mathrm{C}$ NMR $(101 \mathrm{MHz}$, $\left.\mathrm{CDCl}_{3}\right) \delta 197.9,155.3,150.9,148.4,142.8,141.3,137.4,135.7,133.7,129.3,128.8$, 128.1, 125.8, 122.7, 111.7, 110.1, 26.8. IR (KBr), $v\left(\mathrm{~cm}^{-1}\right) 3714,3057,1681,1429,1244,1008,804$, 746; HRMS (ESI) m/z calcd for. $\mathrm{C}_{17} \mathrm{H}_{13} \mathrm{NO}_{2}[\mathrm{M}+\mathrm{H}]^{+} 264.1019$, found 264.1015.

\section{3-(Furan-2-yl)-2-(naphthalen-2-yl)pyridine (10)}

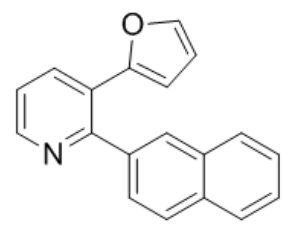

Yield: 58\% (157 mg). Yellow oil. Purified by flash column chromatography $($ EtOAc/hexane $=1 / 40) .{ }^{1} \mathrm{H} \mathrm{NMR}\left(400 \mathrm{MHz}, \mathrm{CDCl}_{3}\right) \delta 8.65(\mathrm{dd}, J=4.7,1.7 \mathrm{~Hz}, 1 \mathrm{H})$, $8.12(\mathrm{dd}, J=7.9,1.7 \mathrm{~Hz}, 1 \mathrm{H}), 8.02(\mathrm{~s}, 1 \mathrm{H}), 7.87(\mathrm{dd}, J=10.2,5.2 \mathrm{~Hz}, 3 \mathrm{H}), 7.56-$ $7.47(\mathrm{~m}, 3 \mathrm{H}), 7.42-7.33(\mathrm{~m}, 2 \mathrm{H}), 6.25(\mathrm{dd}, J=3.4,1.8 \mathrm{~Hz}, 1 \mathrm{H}), 5.83(\mathrm{~d}, J=3.4 \mathrm{~Hz}$, 1H). ${ }^{13} \mathrm{C} \mathrm{NMR}\left(101 \mathrm{MHz}, \mathrm{CDCl}_{3}\right) \delta 156.2,151.1,148.2,142.6,138.5,135.3,133.6,133.3,128.7,128.3$, 128.0, 127.8, 126.8, 126.5, 126.2, 125.9, 122.4, 111.7, 110.3. IR (KBr), v (cm $\left.{ }^{-1}\right) 3743,3053,2229$, 1770, 1434, 1008, 744; HRMS (ESI) m/z calcd for. $\mathrm{C}_{19} \mathrm{H}_{13} \mathrm{NO}[\mathrm{M}+\mathrm{H}]^{+} 272.1070$, found 272.1065.

\section{4-(3-(Furan-2-yl)-5-methylpyridin-2-yl)benzonitrile (1p)}

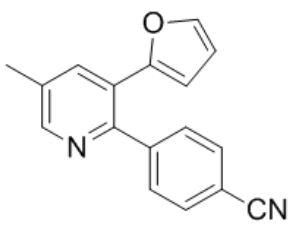

Yield: $65 \%$ (169 mg). White solid. m.p. $126.8-127.7{ }^{\circ} \mathrm{C}$. Purified by flash column chromatography $($ EtOAc/hexane $=1 / 40) .{ }^{1} \mathrm{H}$ NMR $\left(400 \mathrm{MHz}, \mathrm{CDCl}_{3}\right) \delta 9.45(\mathrm{~d}, J=$ $0.9 \mathrm{~Hz}, 1 \mathrm{H}), 8.84(\mathrm{~d}, J=0.9 \mathrm{~Hz}, 1 \mathrm{H}), 8.66(\mathrm{~d}, J=8.3 \mathrm{~Hz}, 2 \mathrm{H}), 8.53(\mathrm{~d}, J=8.3 \mathrm{~Hz}$,

2H), $8.37(\mathrm{~d}, J=1.0 \mathrm{~Hz}, 1 \mathrm{H}), 7.35(\mathrm{dd}, J=3.3,1.8 \mathrm{~Hz}, 1 \mathrm{H}), 6.99(\mathrm{~d}, J=3.3 \mathrm{~Hz}$, $1 \mathrm{H}), 3.43(\mathrm{~s}, 3 \mathrm{H}) .{ }^{13} \mathrm{C} \mathrm{NMR}\left(101 \mathrm{MHz}, \mathrm{CDCl}_{3}\right) \delta 151.6,150.7,149.2,145.4,142.9,136.4,132.9,132.2$, 129.9, 125.2, 119.0, 111.8, 111.8, 110.1, 18.2. IR (KBr), $v\left(\mathrm{~cm}^{-1}\right)$ 3699, 2918, 2223, 1681, 1444, 1016, 752, 584; HRMS (ESI) m/z calcd for. $\mathrm{C}_{17} \mathrm{H}_{12} \mathrm{~N}_{2} \mathrm{O}[\mathrm{M}+\mathrm{Na}]^{+} 283.0842$, found 283.0836. 


\section{4-(5-Methyl-3-(5-methylthiophen-2-yl)pyridin-2-yl)benzonitrile (1q)}

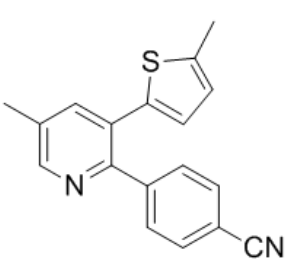

Yield: 67\% (194 mg). White solid. m.p. 152.3-153.4 ${ }^{\circ} \mathrm{C}$. Purified by flash column chromatography $($ EtOAc/hexane $=1 / 30) .{ }^{1} \mathrm{H}$ NMR $\left(400 \mathrm{MHz}, \mathrm{CDCl}_{3}\right) \delta 8.46(\mathrm{~d}, J$ $=1.4 \mathrm{~Hz}, 1 \mathrm{H}), 7.58(\mathrm{dd}, J=16.0,8.4 \mathrm{~Hz}, 5 \mathrm{H}), 6.61-6.56(\mathrm{~m}, 1 \mathrm{H}), 6.52(\mathrm{~d}, J=3.5$ $\mathrm{Hz}, 1 \mathrm{H}), 2.44(\mathrm{~s}, 3 \mathrm{H}), 2.41(\mathrm{~s}, 3 \mathrm{H}) .{ }^{13} \mathrm{C} \mathrm{NMR}\left(101 \mathrm{MHz}, \mathrm{CDCl}_{3}\right) \delta 152.5,149.2$, $145.0,141.8,139.3,137.9,132.9,131.9,130.4,129.3,127.8,125.9,119.1,111.6$,

18.1, 15.4. IR (KBr), $v\left(\mathrm{~cm}^{-1}\right) 3408,3039,2218,1726,1436,1049,802,561$; HRMS (ESI) m/z calcd for. $\mathrm{C}_{18} \mathrm{H}_{14} \mathrm{~N}_{2} \mathrm{~S}[\mathrm{M}+\mathrm{H}]^{+} 291.0950$, found 291.0949 .

\section{1-(4-(3-(Furan-2-yl)-5-methylpyridin-2-yl)phenyl)ethan-1-one (1r)}

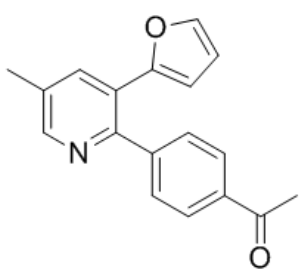

Yield: 55\% (152 mg). Yellow solid. m.p. $111.5-113.7^{\circ} \mathrm{C}$. Purified by flash column chromatography $($ EtOAc/hexane $=1 / 40) .{ }^{1} \mathrm{H}$ NMR $\left(600 \mathrm{MHz}, \mathrm{CDCl}_{3}\right) \delta 8.44(\mathrm{~d}, J$ $=1.5 \mathrm{~Hz}, 1 \mathrm{H}), 7.98(\mathrm{~d}, J=8.3 \mathrm{~Hz}, 2 \mathrm{H}), 7.86(\mathrm{~d}, J=1.3 \mathrm{~Hz}, 1 \mathrm{H}), 7.53(\mathrm{~d}, J=8.3$ $\mathrm{Hz}, 2 \mathrm{H}), 7.37(\mathrm{~d}, J=1.3 \mathrm{~Hz}, 1 \mathrm{H}), 6.31(\mathrm{dd}, J=3.4,1.8 \mathrm{~Hz}, 1 \mathrm{H}), 5.91(\mathrm{~d}, J=3.4$ $\mathrm{Hz}, 1 \mathrm{H}), 2.63$ (s, 3H), 2.42 (s, 3H). ${ }^{13} \mathrm{C} \mathrm{NMR}\left(151 \mathrm{MHz}, \mathrm{CDCl}_{3}\right) \delta 198.1,152.5$, 150.9, 148.9, 145.6, 142.7, 136.6, 136.1, 132.5, 129.3, 128.5, 125.2, 111.7, 110.1, 26.8, 18.2. IR (KBr), $v\left(\mathrm{~cm}^{-1}\right)$ 3452, 2921, 2227, 1949, 1674, 1267, 1012, 759, 597; HRMS (ESI) m/z calcd for. $\mathrm{C}_{18} \mathrm{H}_{15} \mathrm{NO}_{2}$ $[\mathrm{M}+\mathrm{H}]^{+} 278.1176$, found 278.1168 .

\section{2-(Benzofuran-2-yl)-3-phenylpyridine (1s)}

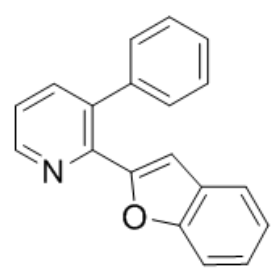

Yield: 57\% (154 mg). Colorless oil. Purified by flash column chromatography $($ EtOAc/hexane $=1 / 40) .{ }^{1} \mathrm{H} \mathrm{NMR}\left(400 \mathrm{MHz}, \mathrm{CDCl}_{3}\right) \delta 8.75(\mathrm{dd}, J=4.6,1.0 \mathrm{~Hz}$, 1H), $7.60(\mathrm{dd}, J=7.5,1.0 \mathrm{~Hz}, 1 \mathrm{H}), 7.49(\mathrm{~d}, J=8.3 \mathrm{~Hz}, 1 \mathrm{H}), 7.44-7.36(\mathrm{~m}, 4 \mathrm{H})$, $7.34-7.22(\mathrm{~m}, 4 \mathrm{H}), 7.14(\mathrm{~d}, J=7.5 \mathrm{~Hz}, 1 \mathrm{H}), 6.27(\mathrm{~s}, 1 \mathrm{H}) .{ }^{13} \mathrm{C}$ NMR $(101 \mathrm{MHz}$, $\left.\mathrm{CDCl}_{3}\right) \delta 154.6,153.6,148.6,146.7,139.5,138.7,136.3,128.9,128.7,128.3,128.1,125.2,122.9$, 122.4, 121.5, 111.7, 108.5. IR (KBr), $v\left(\mathrm{~cm}^{-1}\right)$ 3741, 3053, 1776, 1411, 1257, 1035, 750; HRMS (ESI) $\mathrm{m} / \mathrm{z}$ calcd for. $\mathrm{C}_{19} \mathrm{H}_{13} \mathrm{NO}[\mathrm{M}+\mathrm{H}]^{+} 272.1070$, found 272.1067 .

\section{2-(3-Methylbenzofuran-2-yl)-3-phenylpyridine (1t)}

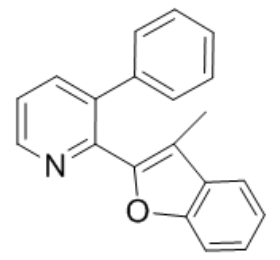

Yield: 67\% (173 mg). Colorless oil. Purified by flash column chromatography $($ EtOAc/hexane $=1 / 40) .{ }^{1} \mathrm{H}$ NMR $\left(400 \mathrm{MHz}, \mathrm{CDCl}_{3}\right) \delta 8.79-8.70(\mathrm{~m}, 1 \mathrm{H}), 7.80$ $(\mathrm{dd}, J=7.8,1.2 \mathrm{~Hz}, 1 \mathrm{H}), 7.43(\mathrm{~d}, J=7.5 \mathrm{~Hz}, 1 \mathrm{H}), 7.40-7.34(\mathrm{~m}, 2 \mathrm{H}), 7.29-7.23$ $(\mathrm{m}, 6 \mathrm{H}), 7.19(\mathrm{t}, J=7.4 \mathrm{~Hz}, 1 \mathrm{H}), 1.88(\mathrm{~s}, 3 \mathrm{H}) .{ }^{13} \mathrm{C} \mathrm{NMR}\left(101 \mathrm{MHz}, \mathrm{CDCl}_{3}\right) \delta 154.5$, $149.7,148.5,148.1,139.4,138.5,137.7,130.2$, 128.8, 128.5, 127.5, 124.8, 123.2, 122.4, 119.8, 115.1, 111.5, 8.5. IR (KBr), $v\left(\mathrm{~cm}^{-1}\right) 3679,3051,1421,1078,750 ; \mathrm{C}_{20} \mathrm{H}_{15} \mathrm{NO}[\mathrm{M}+\mathrm{H}]^{+} 286.1226$, found 286.1231 .

\section{3-(Furan-2-yl)-4-phenylpyridine (3a)}




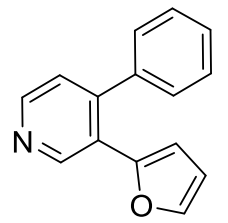

Yield: 65\% (151mg). Yellow oil. Purified by flash column chromatography (EtOAc/ hexane = 1/40). ${ }^{1} \mathrm{H}$ NMR $\left(400 \mathrm{MHz}, \mathrm{CDCl}_{3}\right) \delta 9.00(\mathrm{~s}, 1 \mathrm{H}), 8.53(\mathrm{~d}, J=5.0 \mathrm{~Hz}, 1 \mathrm{H})$, $7.44-7.39(\mathrm{~m}, 3 \mathrm{H}), 7.38(\mathrm{~d}, J=1.8 \mathrm{~Hz}, 1 \mathrm{H}), 7.29-7.25(\mathrm{~m}, 2 \mathrm{H}), 7.20$ (d, $J=5.0 \mathrm{~Hz}$,

$1 \mathrm{H}), 6.28(\mathrm{dd}, J=3.4,1.8 \mathrm{~Hz}, 1 \mathrm{H}), 5.81(\mathrm{~d}, J=3.4 \mathrm{~Hz}, 1 \mathrm{H}) .{ }^{13} \mathrm{C}$ NMR $(101 \mathrm{MHz}$, $\left.\mathrm{CDCl}_{3}\right) \delta 150.2,148.4,148.3,142.5,128.7,128.4,128.4,125.4,124.9,111.5,110.5 . \mathrm{IR}(\mathrm{KBr}), v\left(\mathrm{~cm}^{-1}\right)$ 3440, 3055, 1402, 1080, 744; HRMS (ESI) m/z calcd for. $\mathrm{C}_{15} \mathrm{H}_{11} \mathrm{NO}[\mathrm{M}+\mathrm{H}]^{+} 222.0913$, found 222.0910 .

\section{3-(Furan-2-yl)-4-(p-tolyl)pyridine (3b)}

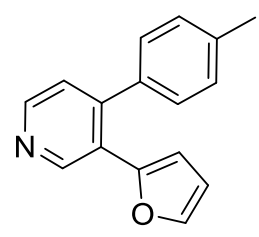

Yield: 42\% (103mg). Colorless oil. Purified by flash column chromatography (EtOAc/ hexane $=1 / 40) .{ }^{1} \mathrm{H}$ NMR $\left(400 \mathrm{MHz}, \mathrm{CDCl}_{3}\right) \delta 8.98(\mathrm{~s}, 1 \mathrm{H}), 8.51(\mathrm{~d}, J=5.0 \mathrm{~Hz}, 1 \mathrm{H})$, $7.19(\mathrm{dt}, J=12.4,8.1 \mathrm{~Hz}, 6 \mathrm{H}), 6.29(\mathrm{dd}, J=3.3,1.8 \mathrm{~Hz}, 1 \mathrm{H}), 5.86(\mathrm{~d}, J=3.3 \mathrm{~Hz}, 1 \mathrm{H})$,

$2.40(\mathrm{~s}, 3 \mathrm{H}) .{ }^{13} \mathrm{C} \mathrm{NMR}\left(101 \mathrm{MHz}, \mathrm{CDCl}_{3}\right) \delta 150.3,148.4,148.3,146.6,142.4,138.3$, 136.3, 129.38 , 128.3, 125.4, 125.0, 111.4, 110.4, 21.4. IR (KBr), $v\left(\mathrm{~cm}^{-1}\right) 3448,1589,1078,815,551$; HRMS (ESI) m/z calcd for. $\mathrm{C}_{16} \mathrm{H}_{13} \mathrm{NO}[\mathrm{M}+\mathrm{H}]^{+} 236.1070$, found 236.1065.

\section{1-(4-(3-(Furan-2-yl)pyridin-4-yl)phenyl)ethan-1-one (3c)}

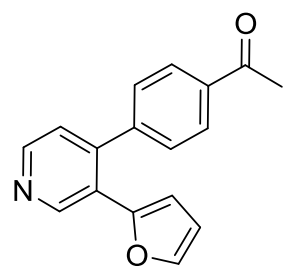

Yield: $30 \%$ (82mg). White solid. m.p. 146-148 ${ }^{\circ} \mathrm{C}$. Purified by flash column chromatography $($ EtOAc/hexane $=1 / 40) .{ }^{1} \mathrm{H}$ NMR $\left(400 \mathrm{MHz}, \mathrm{CDCl}_{3}\right) \delta 9.00(\mathrm{~d}, J=$ $0.7 \mathrm{~Hz}, 1 \mathrm{H}), 8.58(\mathrm{~d}, J=5.1 \mathrm{~Hz}, 1 \mathrm{H}), 8.01(\mathrm{~s}, 1 \mathrm{H}), 8.00(\mathrm{~d}, J=1.7 \mathrm{~Hz}, 1 \mathrm{H}), 7.39$ (s, $1 \mathrm{H}), 7.37(\mathrm{q}, J=1.1 \mathrm{~Hz}, 2 \mathrm{H}), 7.21(\mathrm{dd}, J=5.1,0.7 \mathrm{~Hz}, 1 \mathrm{H}), 6.33-6.30(\mathrm{~m}, 1 \mathrm{H})$, $5.94-5.91(\mathrm{~m}, 1 \mathrm{H}), 2.65(\mathrm{~s}, 3 \mathrm{H}) .{ }^{13} \mathrm{C} \mathrm{NMR}\left(101 \mathrm{MHz}, \mathrm{CDCl}_{3}\right) \delta 197.7,149.9,148.8,148.6,145.4$, $144.1,143.0,128.9,128.7,125.3,124.6,111.6,110.7,26.8$. IR (KBr), v (cm $\left.{ }^{-1}\right) 3446,1591,1085$; HRMS (ESI) $\mathrm{m} / \mathrm{z}$ calcd for. $\mathrm{C}_{17} \mathrm{H}_{13} \mathrm{NO}_{2}[\mathrm{M}+\mathrm{H}]^{+} 264.1019$, found 264.1011.

\section{3-(5-Methylthiophen-2-yl)-4-phenylpyridine (3d)}

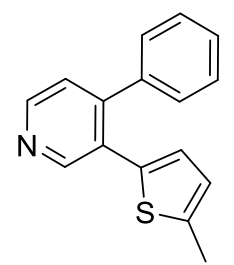

Yield: $70 \%$ (163mg). Yellow oil. Purified by flash column chromatography (EtOAc/ hexane $=1 / 40) .{ }^{1} \mathrm{H}$ NMR $(400 \mathrm{MHz}, \mathrm{CDCl} 3) \delta 8.73(\mathrm{~s}, 1 \mathrm{H}), 8.51(\mathrm{~d}, J=5.0 \mathrm{~Hz}, 1 \mathrm{H})$, $7.33(\mathrm{~d}, J=2.7 \mathrm{~Hz}, 2 \mathrm{H}), 7.32(\mathrm{~d}, J=1.1 \mathrm{~Hz}, 1 \mathrm{H}), 7.26(\mathrm{~d}, J=1.7 \mathrm{~Hz}, 1 \mathrm{H}), 7.24(\mathrm{~d}, J=$ $3.7 \mathrm{~Hz}, 1 \mathrm{H}), 7.21(\mathrm{~d}, J=5.0 \mathrm{~Hz}, 1 \mathrm{H}), 6.58(\mathrm{~d}, J=3.5 \mathrm{~Hz}, 1 \mathrm{H}), 6.55(\mathrm{~d}, J=3.5 \mathrm{~Hz}, 1 \mathrm{H})$, 2.38 (s, 3H). ${ }^{13} \mathrm{C}$ NMR (101 MHz, CDCl3) $\delta 150.5,150.5,148.3,147.5,141.3,138.7,136.5,129.4$, 129.0, 128.4, 128.1, 127.6, 125.6, 124.7, 15.3. IR (KBr), $v\left(\mathrm{~cm}^{-1}\right) 3450,3065,1425,1060,866,740$; HRMS (ESI) m/z calcd for. $\mathrm{C}_{16} \mathrm{H}_{13} \mathrm{NS}[\mathrm{M}+\mathrm{H}]^{+} 252.0841$, found 252.0842 .

\section{4-(Furan-2-yl)-3-phenylpyridine (4a)}

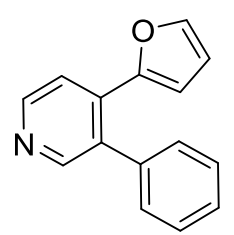

Yield: 77\% (171mg). Colorless oil. Purified by flash column chromatography (EtOAc/ hexane $=1 / 40) .{ }^{1} \mathrm{H}$ NMR $\left(400 \mathrm{MHz}, \mathrm{CDCl}_{3}\right) \delta 8.57(\mathrm{~d}, J=5.3 \mathrm{~Hz}, 1 \mathrm{H}), 8.44(\mathrm{~s}, 1 \mathrm{H})$, $7.69(\mathrm{~d}, J=5.3 \mathrm{~Hz}, 1 \mathrm{H}), 7.48-7.31(\mathrm{~m}, 4 \mathrm{H}), 7.28(\mathrm{~d}, J=3.8 \mathrm{~Hz}, 1 \mathrm{H}), 6.27-6.13(\mathrm{~m}$, $1 \mathrm{H}), 5.70(\mathrm{~d}, J=3.5 \mathrm{~Hz}, 1 \mathrm{H}) .{ }^{13} \mathrm{C} \mathrm{NMR}\left(101 \mathrm{MHz}, \mathrm{CDCl}_{3}\right) \delta 151.0,150.0,148.9,143.0$, 
138.4, 135.7, 133.1, 129.1, 128.7, 128.0, 119.3 , 112.4, 111.8. IR (KBr), v (cm $\left.{ }^{-1}\right) 3452,1635,1083,744$, 561; HRMS (ESI) m/z calcd for. $\mathrm{C}_{15} \mathrm{H}_{11} \mathrm{NO}[\mathrm{M}+\mathrm{Na}]^{+} 244.0733$, found 244.0729.

\section{4-(Furan-2-yl)-3-(4-methoxyphenyl)pyridine (4b)}

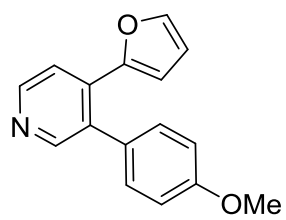

Yield: 67\% (149mg). Colorless oil. Purified by flash column chromatography $($ EtOAc/hexane $=1 / 40) .{ }^{1} \mathrm{H}$ NMR $\left(400 \mathrm{MHz}, \mathrm{CDCl}_{3}\right) \delta 8.63-8.53(\mathrm{~m}, 1 \mathrm{H}), 8.45(\mathrm{~s}$, $1 \mathrm{H}), 7.70(\mathrm{~d}, J=5.3 \mathrm{~Hz}, 1 \mathrm{H}), 7.47-7.31(\mathrm{~m}, 1 \mathrm{H}), 7.24-7.19(\mathrm{~m}, 2 \mathrm{H}), 7.00-6.94$ $(\mathrm{m}, 2 \mathrm{H}), 6.26(\mathrm{~s}, 1 \mathrm{H}), 5.79(\mathrm{~d}, J=3.5 \mathrm{~Hz}, 1 \mathrm{H}), 3.85(\mathrm{~d}, J=1.0 \mathrm{~Hz}, 3 \mathrm{H}) .{ }^{13} \mathrm{C} \mathrm{NMR}$ $\left(101 \mathrm{MHz}, \mathrm{CDCl}_{3}\right) \delta 159.5,151.3,150.2,148.7,143.0,136.0,133.0,130.6,130.3,119.3,114.2,112.4$, 111.9, 55.3. IR (KBr), $v\left(\mathrm{~cm}^{-1}\right) 3450,1631,1114,611$; HRMS (ESI) m/z calcd for. $\mathrm{C}_{16} \mathrm{H}_{13} \mathrm{NO}_{2}[\mathrm{M}+\mathrm{H}]$ ${ }^{+} 252.1019$, found 252.1010 .

\section{4-(Furan-2-yl)-3-(p-tolyl)pyridine (4c)}

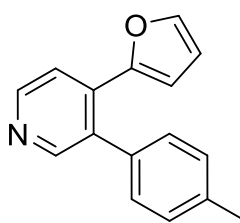

Yield: $75 \%$ (167mg). Colorless oil. Purified by flash column chromatography $($ EtOAc/hexane $=1 / 40) .{ }^{1} \mathrm{H} \mathrm{NMR}\left(400 \mathrm{MHz}, \mathrm{CDCl}_{3}\right) \delta 8.58(\mathrm{~d}, J=5.3 \mathrm{~Hz}, 1 \mathrm{H}), 8.45(\mathrm{~s}$, $1 \mathrm{H}), 7.71(\mathrm{~d}, J=5.2 \mathrm{~Hz}, 1 \mathrm{H}), 7.42(\mathrm{~s}, 1 \mathrm{H}), 7.25(\mathrm{~d}, J=7.5 \mathrm{~Hz}, 2 \mathrm{H}), 7.19(\mathrm{~d}, J=7.8 \mathrm{~Hz}$, 2H), $6.35-6.19(\mathrm{~m}, 1 \mathrm{H}), 5.77(\mathrm{~d}, J=3.5 \mathrm{~Hz}, 1 \mathrm{H}), 2.43(\mathrm{~s}, 3 \mathrm{H}) .{ }^{13} \mathrm{C}$ NMR $(101 \mathrm{MHz}$, $\left.\mathrm{CDCl}_{3}\right) \delta 151.3,150.2,148.8,143.1,138.0,136.1,135.5,133.4,129.6,129.1,119.4,112.6,112.0,21.4$. IR (KBr), $v\left(\mathrm{~cm}^{-1}\right) 3051,2947,2530,1743,1463,1242,1020,840,565$; HRMS (ESI) m/z calcd for. $\mathrm{C}_{16} \mathrm{H}_{13} \mathrm{NO}[\mathrm{M}+\mathrm{H}]^{+} 236.1070$, found 236.1063.

\section{4-(Furan-2-yl)-3-(4-(trifluoromethyl)phenyl)pyridine (4d)}

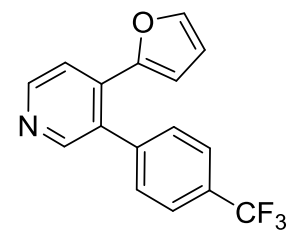

Yield: 90\% (200mg). Yellow oil. Purified by flash column chromatography (EtOAc/ hexane = 1/40). ${ }^{1} \mathrm{H}$ NMR $\left(400 \mathrm{MHz}, \mathrm{CDCl}_{3}\right) \delta 8.64(\mathrm{~d}, J=5.3 \mathrm{~Hz}, 1 \mathrm{H}), 8.45(\mathrm{~s}, 1 \mathrm{H})$, $7.77-7.67(\mathrm{~m}, 3 \mathrm{H}), 7.48-7.37(\mathrm{~m}, 3 \mathrm{H}), 6.37-6.25(\mathrm{~m}, 1 \mathrm{H}), 5.81(\mathrm{~d}, J=3.6 \mathrm{~Hz}$,

$1 \mathrm{H}) .{ }^{13} \mathrm{C} \mathrm{NMR}\left(101 \mathrm{MHz}, \mathrm{CDCl}_{3}\right) \delta 151.0,149.9,149.7,143.6,142.4,135.9,132.0$, 130.5(q, $\left.{ }^{2} \mathrm{~J}=32 \mathrm{~Hz}\right), 129.9,125.8\left(\mathrm{q},{ }^{3} \mathrm{~J}=4.0 \mathrm{~Hz}\right), 124.3\left(\mathrm{q},{ }^{1} \mathrm{~J}=270 \mathrm{~Hz}\right), 112.6,112.1$. IR (KBr), $v(\mathrm{~cm}$ ${ }^{-1}$ )3747, 2920, 1776, 1579, 997, 811; HRMS (ESI) m/z calcd for. $\mathrm{C}_{16} \mathrm{H}_{10} \mathrm{~F}_{3} \mathrm{NO}[\mathrm{M}+\mathrm{H}]^{+} 290.0787$, found 290.0778 .

\section{1-(4-(4-(Furan-2-yl)pyridin-3-yl)phenyl)ethan-1-one (4e)}

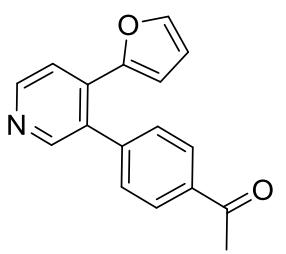

Yield: $70 \%$ (156mg). White solid. m.p. $112-113^{\circ} \mathrm{C}$. Purified by flash column chromatography $($ EtOAc/hexane $=1 / 40) .{ }^{1} \mathrm{H}$ NMR $\left(400 \mathrm{MHz}, \mathrm{CDCl}_{3}\right) \delta 8.62(\mathrm{~d}, J=$ $5.2 \mathrm{~Hz}, 1 \mathrm{H}), 8.45(\mathrm{~s}, 1 \mathrm{H}), 8.11-7.98(\mathrm{~m}, 2 \mathrm{H}), 7.71(\mathrm{~d}, J=5.3 \mathrm{~Hz}, 1 \mathrm{H}), 7.49-7.28$ $(\mathrm{m}, 3 \mathrm{H}), 6.29(\mathrm{dd}, J=3.5,1.8 \mathrm{~Hz}, 1 \mathrm{H}), 5.85(\mathrm{~d}, J=3.5 \mathrm{~Hz}, 1 \mathrm{H}), 2.67(\mathrm{~s}, 3 \mathrm{H}) .{ }^{13} \mathrm{C}$ $\operatorname{NMR}\left(101 \mathrm{MHz}, \mathrm{CDCl}_{3}\right) \delta 197.5,150.6,149.7,149.4,143.3,136.5,135.6,132.1,129.5,128.6,119.6$, 112.4, 111.9, 26.6. IR (KBr), $v\left(\mathrm{~cm}^{-1}\right)$ 3768, 3377, 3161, 2931, 2422, 2376, 1926, 1697, 1404, 1267 , 1020, 759, 599; HRMS (ESI) m/z calcd for. $\mathrm{C}_{17} \mathrm{H}_{13} \mathrm{NO}_{2}[\mathrm{M}+\mathrm{H}]^{+} 264.1019$, found 264.1010. 


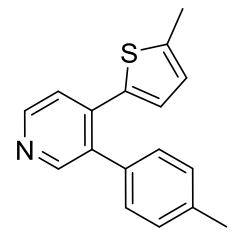

Yield: $86 \%$ (217mg). Colorless oil. Purified by flash column chromatography (EtOAc/ hexane $=1 / 40) .{ }^{1} \mathrm{H}$ NMR $\left(400 \mathrm{MHz}, \mathrm{CDCl}_{3}\right) \delta 8.52(\mathrm{~d}, J=5.1 \mathrm{~Hz}, 1 \mathrm{H}), 8.49(\mathrm{~s}, 1 \mathrm{H})$, $7.40(\mathrm{~d}, J=5.1 \mathrm{~Hz}, 1 \mathrm{H}), 7.19$ (s, 4H), $6.66(\mathrm{~d}, J=3.5 \mathrm{~Hz}, 1 \mathrm{H}), 6.57$ (dd, $J=3.6,1.3 \mathrm{~Hz}$,

$1 \mathrm{H}), 2.42(\mathrm{~d}, J=1.1 \mathrm{~Hz}, 3 \mathrm{H}), 2.41(\mathrm{~s}, 3 \mathrm{H}) .{ }^{13} \mathrm{C} \mathrm{NMR}\left(101 \mathrm{MHz}, \mathrm{CDCl}_{3}\right) \delta 151.6,148.6$, $142.4,140.6,137.7,135.2,134.9,129.7,129.4,128.5,125.9,123.0,21.4,15.4$. IR (KBr), $v\left(\mathrm{~cm}^{-1}\right) 3660$, 3002, 1801, 1656, 1264, 1032, 622; HRMS (ESI) m/z calcd for. $\mathrm{C}_{17} \mathrm{H}_{15} \mathrm{NS}[\mathrm{M}+\mathrm{H}]^{+} 266.0998$, found 266.1005 .

\section{1-(5-(3-(p-tolyl)Pyridin-4-yl)thiophen-2-yl)ethan-1-one (4g)}

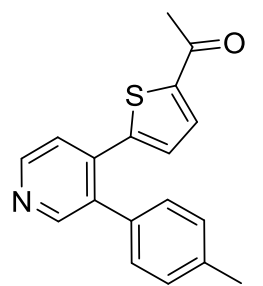

Yield: $91 \%$ (255mg). White solid. m.p. $145.1-146.8^{\circ} \mathrm{C}$. Purified by flash column chromatography $($ EtOAc/hexane $=1 / 40) .{ }^{1} \mathrm{H}$ NMR $\left(400 \mathrm{MHz}, \mathrm{CDCl}_{3}\right) \delta 8.62-8.56(\mathrm{~m}$, 2H), $7.43(\mathrm{dd}, \mathrm{J}=10.2,4.6 \mathrm{~Hz}, 2 \mathrm{H}), 7.17(\mathrm{~s}, 1 \mathrm{H}), 7.15(\mathrm{~s}, 1 \mathrm{H}), 7.11(\mathrm{~d}, \mathrm{~J}=8.2 \mathrm{~Hz}, 2 \mathrm{H})$, $6.77(\mathrm{~d}, \mathrm{~J}=3.9 \mathrm{~Hz}, 1 \mathrm{H}), 2.47(\mathrm{~s}, 3 \mathrm{H}), 2.36(\mathrm{~s}, 3 \mathrm{H}) \cdot{ }^{13} \mathrm{C} \mathrm{NMR}\left(101 \mathrm{MHz}, \mathrm{CDCl}_{3}\right) \delta$ $190.5,151.3,148.5,148.2$, 145.2, 139.5, 138.2, 135.7, 134.0, 132.5, 129.5, 129.5, 129.2, 123.6, 26.7, 21.3. IR (KBr), $v\left(\mathrm{~cm}^{-1}\right) 3625,3030,1901,1656,1274,1033,811,657$; HRMS (ESI) m/z calcd for. $\mathrm{C}_{18} \mathrm{H}_{15} \mathrm{NOS}[\mathrm{M}+\mathrm{H}]^{+}$294.0947, found 294.0949.

\section{4-(Furan-2-yl)-3-(o-tolyl)pyridine (4h)}

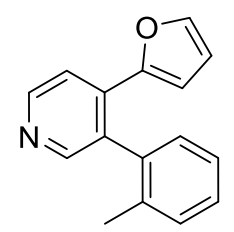

Yield: $80 \%$ (178mg). White solid. m.p. $76-77.8^{\circ} \mathrm{C}$. Purified by flash column chromatography $($ EtOAc/hexane $=1 / 40) .{ }^{1} \mathrm{H}$ NMR $\left(400 \mathrm{MHz}, \mathrm{CDCl}_{3}\right) \delta 8.62(\mathrm{~d}, J=5.3$ $\mathrm{Hz}, 1 \mathrm{H}), 8.40(\mathrm{~s}, 1 \mathrm{H}), 7.79(\mathrm{~d}, J=5.2 \mathrm{~Hz}, 1 \mathrm{H}), 7.42(\mathrm{~d}, J=1.8 \mathrm{~Hz}, 1 \mathrm{H}), 7.39-7.34(\mathrm{~m}$, $1 \mathrm{H}), 7.33-7.27(\mathrm{~m}, 2 \mathrm{H}), 7.20-7.13(\mathrm{~m}, 1 \mathrm{H}), 6.22(\mathrm{dd}, J=3.6,1.8 \mathrm{~Hz}, 1 \mathrm{H}), 5.48(\mathrm{~d}, J=$ $3.4 \mathrm{~Hz}, 1 \mathrm{H}), 1.99$ (s, 3H). ${ }^{13} \mathrm{C}$ NMR (101 MHz, $\left.\mathrm{CDCl}_{3}\right) \delta 151.0,150.1,149.1,143.1,138.0,136.5,136.0$, 132., 130.4, 129.5, 128.6, 126.5, 118.7, 112.2, 111.9, 19.7. IR (KBr), v (cm $\left.{ }^{-1}\right) 3438,2920,1917,1587$, 1461, 1083, 1012, 738; HRMS (ESI) m/z calcd for. $\mathrm{C}_{16} \mathrm{H}_{13} \mathrm{NO}[\mathrm{M}+\mathrm{H}]^{+} 236.1070$, found 236.1071.

\section{4-(Benzofuran-2-yl)-3-phenylpyridine (4i)}

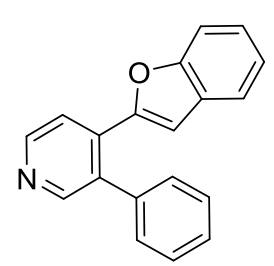

Yield: $90 \%$ (245mg). White solid. m.p. 90.5-91.0 ${ }^{\circ}$ C. Purified by flash column chromatography $($ EtOAc/hexane $=1 / 40) .{ }^{1} \mathrm{H}$ NMR $\left(400 \mathrm{MHz}, \mathrm{CDCl}_{3}\right) \delta 8.69(\mathrm{~d}, J=$ $5.3 \mathrm{~Hz}, 1 \mathrm{H}), 8.54(\mathrm{~s}, 1 \mathrm{H}), 7.93(\mathrm{dd}, J=5.3,0.6 \mathrm{~Hz}, 1 \mathrm{H}), 7.49-7.45(\mathrm{~m}, 4 \mathrm{H}), 7.40-$ $7.35(\mathrm{~m}, 3 \mathrm{H}), 7.29(\mathrm{ddd}, J=8.4,7.2,1.3 \mathrm{~Hz}, 1 \mathrm{H}), 7.19-7.14(\mathrm{~m}, 1 \mathrm{H}), 6.07(\mathrm{~s}, 1 \mathrm{H})$. ${ }^{13} \mathrm{C}$ NMR $\left(101 \mathrm{MHz}, \mathrm{CDCl}_{3}\right) \delta 154.5,151.7,151.3,149.2,138.2,136.0,134.6,129.3,129.0,128.5$, 128.5, 125.7, 123.3, 121.8, 120.6, 111.4, 109.0. IR (KBr), $v\left(\mathrm{~cm}^{-1}\right) 3448,1587,1107,750,603$; HRMS (ESI) $\mathrm{m} / \mathrm{z}$ calcd for. $\mathrm{C}_{19} \mathrm{H}_{13} \mathrm{NO}[\mathrm{M}+\mathrm{H}]^{+} 272.1070$, found 272.1070 .

\section{5-(Furan-2-yl)-4-phenylpyrimidine (7a)}

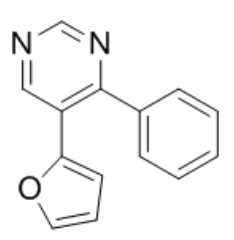

Yield: $52 \%$ (115 mg). White solid. m.p. 72.5-73.8 ${ }^{\circ} \mathrm{C}$. Purified by flash column chromatography $($ EtOAc/hexane $=1 / 40) .{ }^{1} \mathrm{H}$ NMR $\left(400 \mathrm{MHz}, \mathrm{CDCl}_{3}\right) \delta 9.17(\mathrm{~s}, 1 \mathrm{H})$, $9.05(\mathrm{~s}, 1 \mathrm{H}), 7.51(\mathrm{~d}, J=7.6 \mathrm{~Hz}, 2 \mathrm{H}), 7.48-7.42(\mathrm{~m}, 4 \mathrm{H}), 6.37(\mathrm{~s}, 1 \mathrm{H}), 6.08$ (d, $J=3.3$ 
$\mathrm{Hz}, 1 \mathrm{H}) .{ }^{13} \mathrm{C} \mathrm{NMR}\left(101 \mathrm{MHz}, \mathrm{CDCl}_{3}\right) \delta 162.6,157.0,155.8,148.2,143.5,138.2,129.9,128.7,128.6$, 123.4, 111.9, 111.2. IR (KBr), $v\left(\mathrm{~cm}^{-1}\right) 3427,2923,2221,1899,1440,1392,1022,756,698$; HRMS (ESI) $\mathrm{m} / \mathrm{z}$ calcd for. $\mathrm{C}_{14} \mathrm{H}_{10} \mathrm{~N}_{2} \mathrm{O}[\mathrm{M}+\mathrm{H}]^{+} 223.0866$, found 223.0868 .

\section{4-Phenyl-5-(thiophen-2-yl)pyrimidine (7b)}

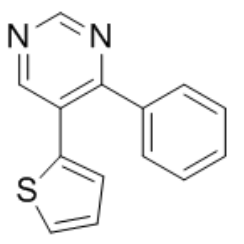

Yield: 57\% (135 mg). Yellow solid. m.p. 89.1-89.8 ${ }^{\circ} \mathrm{C}$. Purified by flash column chromatography $($ EtOAc/hexane $=1 / 40) .{ }^{1} \mathrm{H}$ NMR $\left(400 \mathrm{MHz}, \mathrm{CDCl}_{3}\right) \delta 9.21(\mathrm{~s}, 1 \mathrm{H})$, $8.83(\mathrm{~s}, 1 \mathrm{H}), 7.51(\mathrm{dd}, J=5.2,3.1 \mathrm{~Hz}, 2 \mathrm{H}), 7.41-7.33(\mathrm{~m}, 4 \mathrm{H}), 7.00(\mathrm{dd}, J=5.1,3.6$ $\mathrm{Hz}, 1 \mathrm{H}), 6.93(\mathrm{dd}, J=3.5,1.1 \mathrm{~Hz}, 1 \mathrm{H}) .{ }^{13} \mathrm{C} \mathrm{NMR}\left(101 \mathrm{MHz}, \mathrm{CDCl}_{3}\right) \delta 163.9,158.1$, 157.6, 137.4, 137.1, 129.8, 129.5, 128.5, 128.2, 127.8, 127.7, 127.2. IR (KBr), v (cm $\left.{ }^{-1}\right) 3708,3057$, 1677, 1436, 1271, 844, 698; HRMS (ESI) m/z calcd for. $\mathrm{C}_{14} \mathrm{H}_{10} \mathrm{~N}_{2} \mathrm{~S}[\mathrm{M}+\mathrm{H}]^{+}$239.0637, found 239.0639.

\section{5-(5-Methylfuran-2-yl)-4-phenylpyrimidine (7c)}

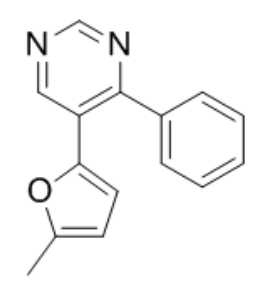

Yield: 59\% (139 mg). Yellow oil. Purified by flash column chromatography $($ EtOAc/hexane $=1 / 40) .{ }^{1} \mathrm{H}$ NMR $\left(400 \mathrm{MHz}, \mathrm{CDCl}_{3}\right) \delta 9.09(\mathrm{~s}, 1 \mathrm{H}), 9.03(\mathrm{~s}, 1 \mathrm{H}), 7.53$ $-7.47(\mathrm{~m}, 2 \mathrm{H}), 7.41(\mathrm{~d}, J=7.0 \mathrm{~Hz}, 3 \mathrm{H}), 5.91(\mathrm{~s}, 1 \mathrm{H}), 5.87(\mathrm{~s}, 1 \mathrm{H}), 2.26(\mathrm{~s}, 3 \mathrm{H}) .{ }^{13} \mathrm{C}$ NMR $\left(101 \mathrm{MHz}, \mathrm{CDCl}_{3}\right) \delta 161.9,156.3,154.9,153.5,146.2,138.4,129.6,128.6$, 128.5, 123.5, 112.3, 108.0, 13.6. IR (KBr), $v\left(\mathrm{~cm}^{-1}\right) 3427,1572,1436,1131,1076$, 698; HRMS (ESI) m/z calcd for. $\mathrm{C}_{15} \mathrm{H}_{12} \mathrm{~N}_{2} \mathrm{O}[\mathrm{M}+\mathrm{H}]^{+}$237.1022, found 237.1024.

\section{5-(5-Methylthiophen-2-yl)-4-phenylpyrimidine (7d)}

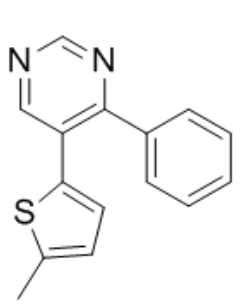

Yield: 55\% (138 mg). White solid. m.p. 83.3-84.8 ${ }^{\circ} \mathrm{C}$. Purified by flash column chromatography $($ EtOAc/hexane $=1 / 40) .{ }^{1} \mathrm{H}$ NMR $\left(400 \mathrm{MHz}, \mathrm{CDCl}_{3}\right) \delta 9.16(\mathrm{~s}, 1 \mathrm{H})$, $8.78(\mathrm{~s}, 1 \mathrm{H}), 7.56-7.52(\mathrm{~m}, 2 \mathrm{H}), 7.37$ (dt, $J=14.1,6.7 \mathrm{~Hz}, 3 \mathrm{H}), 6.70(\mathrm{~d}, J=3.5 \mathrm{~Hz}$, $1 \mathrm{H}), 6.63(\mathrm{dd}, J=3.4,0.8 \mathrm{~Hz}, 1 \mathrm{H}), 2.44(\mathrm{~s}, 3 \mathrm{H}) .{ }^{13} \mathrm{C} \mathrm{NMR}\left(101 \mathrm{MHz}, \mathrm{CDCl}_{3}\right) \delta 163.5$, $157.9,157.1,142.5,137.5,134.5,129.7,129.4,128.4,128.1,127.4,126.1,15.4$. IR $(\mathrm{KBr}), v\left(\mathrm{~cm}^{-1}\right) 3448,3049,1772,1552,1157,752,595$; HRMS (ESI) m/z calcd for. $\mathrm{C}_{15} \mathrm{H}_{12} \mathrm{~N}_{2} \mathrm{~S}$ $[\mathrm{M}+\mathrm{H}]^{+}$253.0794, found 253.0791.

\section{2-([1,1'-biphenyl]-2-yl)furan (9a)}

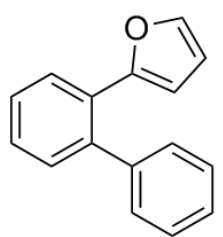

Yield: 75\% (165 mg). Colorless oil. Purified by flash column chromatography (100\% hexane). ${ }^{1} \mathrm{H}$ NMR $\left(400 \mathrm{MHz}, \mathrm{CDCl}_{3}\right) \delta 7.88(\mathrm{~d}, J=7.7 \mathrm{~Hz}, 1 \mathrm{H}), 7.50-7.31(\mathrm{~m}, 9 \mathrm{H})$, $6.28(\mathrm{dd}, J=3.3,1.8 \mathrm{~Hz}, 1 \mathrm{H}), 5.68(\mathrm{~d}, J=3.4 \mathrm{~Hz}, 1 \mathrm{H}) .{ }^{13} \mathrm{C} \mathrm{NMR}\left(101 \mathrm{MHz}, \mathrm{CDCl}_{3}\right) \delta$ $153.0,142.2,141.56,139.6,130.9,129.5,129.2,128.4,127.7,127.4,127.3,127.2$, 111.4, 109.1. IR (KBr), $v\left(\mathrm{~cm}^{-1}\right)$ 3450, 1570, 1123, 750, 606; HRMS (ESI) m/z calcd for. $\mathrm{C}_{16} \mathrm{H}_{12} \mathrm{O}$ $[\mathrm{M}+\mathrm{H}]^{+}$221.0961, found 221.0957. 


\section{The data of $2 a-2 v, 5 a-5 d, 6 a-6 j, 8 a-8 d$.}

\section{Benzo $[h]$ furo $[2,3-f]$ quinolone (2a)}

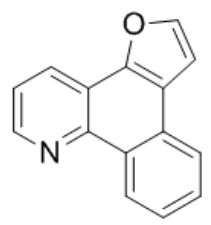

Yield: 60\% (33 mg). White solid. m.p. 97.2-98.9 ${ }^{\circ} \mathrm{C}$. Purified by flash column chromatography $($ EtOAc/hexane $=1 / 20) .{ }^{1} \mathrm{H}$ NMR $\left(400 \mathrm{MHz}, \mathrm{CDCl}_{3}\right) \delta 9.36(\mathrm{~d}, J=8.1$ $\mathrm{Hz}, 1 \mathrm{H}), 9.00(\mathrm{dd}, J=4.4,1.5 \mathrm{~Hz}, 1 \mathrm{H}), 8.60(\mathrm{dd}, J=8.0,1.5 \mathrm{~Hz}, 1 \mathrm{H}), 8.14(\mathrm{~d}, J=7.5 \mathrm{~Hz}$, 1H), $7.86(\mathrm{~d}, J=1.9 \mathrm{~Hz}, 1 \mathrm{H}), 7.80-7.70(\mathrm{~m}, 2 \mathrm{H}), 7.60(\mathrm{dd}, J=8.1,4.4 \mathrm{~Hz}, 1 \mathrm{H}), 7.30(\mathrm{~d}$, $J=1.9 \mathrm{~Hz}, 1 \mathrm{H}) .{ }^{13} \mathrm{C} \mathrm{NMR}\left(101 \mathrm{MHz}, \mathrm{CDCl}_{3}\right) \delta 147.9,147.6,145.5,145.0,129.4,128.8,128.5,128.3$, 125.8, 125.8, 123.5, 122.1, 120.8, 117.7, 106.6. IR (KBr), $v\left(\mathrm{~cm}^{-1}\right) 3452,3101,1940,1566,1398,1332$, 1020, 734, 595; HRMS (ESI) m/z calcd for. $\mathrm{C}_{15} \mathrm{H}_{9} \mathrm{NO}[\mathrm{M}+\mathrm{H}]^{+} 220.0757$, found 220.0756.

\section{Benzo $[h]$ thieno $[2,3-f]$ quinolone $(2 \mathrm{~b})$}

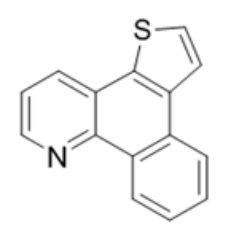

Yield: 58\% (34 mg). White solid. m.p. 146.4-148.7 ${ }^{\circ} \mathrm{C}$. Purified by flash column chromatography $($ EtOAc/hexane $=1 / 20) .{ }^{1} \mathrm{H}$ NMR $\left(400 \mathrm{MHz}, \mathrm{CDCl}_{3}\right) \delta 9.35-9.28(\mathrm{~m}$, $1 \mathrm{H}), 8.96(\mathrm{dd}, J=4.0,1.0 \mathrm{~Hz}, 1 \mathrm{H}), 8.37(\mathrm{dd}, J=8.1,1.1 \mathrm{~Hz}, 1 \mathrm{H}), 8.29-8.25(\mathrm{~m}, 1 \mathrm{H})$, $7.96(\mathrm{~d}, J=5.3 \mathrm{~Hz}, 1 \mathrm{H}), 7.77-7.69(\mathrm{~m}, 2 \mathrm{H}), 7.61(\mathrm{~d}, J=5.3 \mathrm{~Hz}, 1 \mathrm{H}), 7.52(\mathrm{dd}, J=8.0$, $4.5 \mathrm{~Hz}, 1 \mathrm{H}) .{ }^{13} \mathrm{C}$ NMR $\left(101 \mathrm{MHz}, \mathrm{CDCl}_{3}\right) \delta 148.4,145.3,135.6,134.9,131.7,130.2,128.9,126.6$, 126.1, 125.7, 123.8, 123.4, 123.3, 122.2. IR (KBr), $v\left(\mathrm{~cm}^{-1}\right)$ 3699, 3053, 1731, 1411, 960, 723; HRMS (ESI) $\mathrm{m} / \mathrm{z}$ calcd for. $\mathrm{C}_{15} \mathrm{H}_{9} \mathrm{NS}[\mathrm{M}+\mathrm{H}]^{+} 236.0528$, found 236.0520 .

\section{6-Methylbenzo $[h]$ thieno[2,3-f]quinolone (2c)}

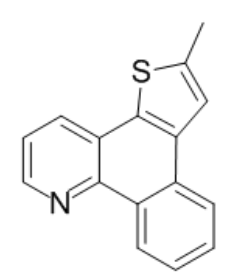

Yield: 52\% (32 mg). White solid. m.p. 157.3-158.9 ${ }^{\circ} \mathrm{C}$. Purified by flash column chromatography $($ EtOAc/hexane $=1 / 20) .{ }^{1} \mathrm{H}$ NMR $\left(400 \mathrm{MHz}, \mathrm{CDCl}_{3}\right) \delta 9.34-9.26(\mathrm{~m}$, $1 \mathrm{H}), 8.92(\mathrm{dd}, J=4.3,1.3 \mathrm{~Hz}, 1 \mathrm{H}), 8.26(\mathrm{dd}, J=8.1,1.4 \mathrm{~Hz}, 1 \mathrm{H}), 8.19$ (d, $J=7.2 \mathrm{~Hz}$,

$1 \mathrm{H}), 7.75-7.67(\mathrm{~m}, 2 \mathrm{H}), 7.60(\mathrm{~s}, 1 \mathrm{H}), 7.50(\mathrm{dd}, J=8.1,4.4 \mathrm{~Hz}, 1 \mathrm{H}), 2.71(\mathrm{~s}, 3 \mathrm{H}) .{ }^{13} \mathrm{C}$ NMR $\left(101 \mathrm{MHz}, \mathrm{CDCl}_{3}\right) \delta 147.8,144.9,141.1,136.0,133.6,131.3,130.2,130.0,128.7,126.3,125.6$, 123.7, 123.4, 122.1, 121.4, 16.4. IR (KBr), $v\left(\mathrm{~cm}^{-1}\right)$ 3417, 2920, 1560, 1411, 1261, 1024, 761; HRMS (ESI) $\mathrm{m} / \mathrm{z}$ calcd for. $\mathrm{C}_{16} \mathrm{H}_{11} \mathrm{NS}[\mathrm{M}+\mathrm{H}]^{+} 250.0685$, found 250.0681 .

\section{9-Methylbenzo[h]furo[2,3-f]quinolone (2d)}

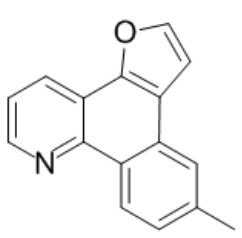

Yield: 62\% (36 mg). Yellow solid. m.p. 109.9-110.7 ${ }^{\circ} \mathrm{C}$. Purified by flash column chromatography $($ EtOAc/hexane $=1 / 20) .{ }^{1} \mathrm{H}$ NMR $\left(600 \mathrm{MHz}, \mathrm{CDCl}_{3}\right) \delta 9.19(\mathrm{~d}, J=$ $8.4 \mathrm{~Hz}, 1 \mathrm{H}), 8.95(\mathrm{dd}, J=4.4,1.7 \mathrm{~Hz}, 1 \mathrm{H}), 8.57(\mathrm{dd}, J=8.0,1.7 \mathrm{~Hz}, 1 \mathrm{H}), 7.91(\mathrm{~s}, 1 \mathrm{H})$, $7.84(\mathrm{~d}, J=2.0 \mathrm{~Hz}, 1 \mathrm{H}), 7.56(\mathrm{dd}, J=8.0,4.4 \mathrm{~Hz}, 1 \mathrm{H}), 7.52(\mathrm{dd}, J=8.4,1.2 \mathrm{~Hz}, 1 \mathrm{H})$, $7.27(\mathrm{~d}, J=2.0 \mathrm{~Hz}, 1 \mathrm{H}), 2.63(\mathrm{~s}, 3 \mathrm{H}) .{ }^{13} \mathrm{C} \mathrm{NMR}\left(151 \mathrm{MHz}, \mathrm{CDCl}_{3}\right) \delta$ 147.9, 147.8, 145.6, 144.9, 138.9, 128.6, 128.2, 127.5, 127.2, 125.7, 123.3, 121.6, 120.6, 117.3, 106.5, 22.0. IR (KBr), v $\left(\mathrm{cm}^{-1}\right) 3689$, 2921, 1726, 1404, 1334, 1028, 754; HRMS (ESI) m/z calcd for. $\mathrm{C}_{16} \mathrm{H}_{11} \mathrm{NO}[\mathrm{M}+\mathrm{H}]^{+} 234.0913$, found 234.0908 .

\section{9-Methoxybenzo[h]furo[2,3-f]quinoline (2e)}




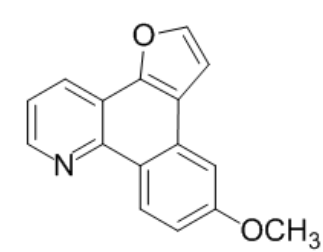

Yield: $62 \%$ (38 mg). White solid. m.p. 110.9-112.3 ${ }^{\circ} \mathrm{C}$. Purified by flash column chromatography $($ EtOAc/hexane $=1 / 20) .{ }^{1} \mathrm{H} \mathrm{NMR}\left(400 \mathrm{MHz}, \mathrm{CDCl}_{3}\right) \delta 9.21(\mathrm{~d}, J$ $=9.1 \mathrm{~Hz}, 1 \mathrm{H}), 8.98-8.87(\mathrm{~m}, 1 \mathrm{H}), 8.53(\mathrm{dd}, J=8.0,1.3 \mathrm{~Hz}, 1 \mathrm{H}), 7.82(\mathrm{~d}, J=1.7$ $\mathrm{Hz}, 1 \mathrm{H}), 7.51(\mathrm{dd}, J=8.0,4.5 \mathrm{~Hz}, 1 \mathrm{H}), 7.46(\mathrm{~d}, J=2.3 \mathrm{~Hz}, 1 \mathrm{H}), 7.29(\mathrm{dd}, J=9.1$, $2.4 \mathrm{~Hz}, 1 \mathrm{H}), 7.22(\mathrm{~d}, J=1.7 \mathrm{~Hz}, 1 \mathrm{H}), 4.01(\mathrm{~s}, 3 \mathrm{H}) .{ }^{13} \mathrm{C} \mathrm{NMR}\left(101 \mathrm{MHz}, \mathrm{CDCl}_{3}\right) \delta 160.3,148.1,148.0$, 145.6, 144.8, 130.0, 128.2, 127.6, 123.5, 121.1, 120.5, 116.5, 115.2, 106.5, 105.0, 55.7. IR (KBr), $v$ $\left(\mathrm{cm}^{-1}\right)$ 3454, 2923, 1907, 1625, 1400, 1230, 1029, 746, 580; HRMS (ESI) m/z calcd for. $\mathrm{C}_{16} \mathrm{H}_{11} \mathrm{NO}_{2}$ $[\mathrm{M}+\mathrm{H}]^{+} 250.0863$, found 250.0858 .

\section{9-Fluorobenzo[ $h]$ furo[2,3-f $f$ quinoline $(2 \mathrm{f})$}

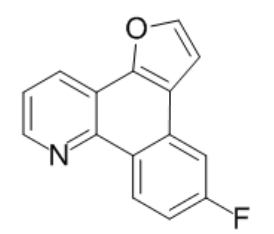

Yield: $70 \%$ (41 mg). White solid. m.p. 123.9-125.4 ${ }^{\circ} \mathrm{C}$. Purified by flash column chromatography $($ EtOAc/hexane $=1 / 20) .{ }^{1} \mathrm{H}$ NMR $\left(400 \mathrm{MHz}, \mathrm{CDCl}_{3}\right) \delta 9.31(\mathrm{dd}, J=$ $9.1,5.9 \mathrm{~Hz}, 1 \mathrm{H}), 9.00-8.93(\mathrm{~m}, 1 \mathrm{H}), 8.56(\mathrm{dd}, J=8.1,1.2 \mathrm{~Hz}, 1 \mathrm{H}), 7.84(\mathrm{~d}, J=1.7$ $\mathrm{Hz}, 1 \mathrm{H}), 7.71(\mathrm{dd}, J=9.4,2.5 \mathrm{~Hz}, 1 \mathrm{H}), 7.57(\mathrm{dd}, J=8.1,4.4 \mathrm{~Hz}, 1 \mathrm{H}), 7.40(\mathrm{td}, J=8.8$, $2.5 \mathrm{~Hz}, 1 \mathrm{H}), 7.21(\mathrm{~d}, J=1.7 \mathrm{~Hz}, 1 \mathrm{H}) .{ }^{13} \mathrm{C} \mathrm{NMR}\left(101 \mathrm{MHz}, \mathrm{CDCl}_{3}\right) \delta 164.4,162.0,148.2,146.7\left(\mathrm{~d},{ }^{1} J=\right.$ $304 \mathrm{~Hz}), 145.1,130.0\left(\mathrm{~d},{ }^{3} \mathrm{~J}=9.8 \mathrm{~Hz}\right), 128.5\left(\mathrm{~d},{ }^{3} \mathrm{~J}=9.3 \mathrm{~Hz}\right), 128.4,125.9,121.9,120.3\left(\mathrm{~d},{ }^{4} \mathrm{~J}=3.5 \mathrm{~Hz}\right)$, 117.1, $114.4\left(\mathrm{~d},{ }^{2} J=23 \mathrm{~Hz}\right), 108.5\left(\mathrm{~d},{ }^{2} J=22 \mathrm{~Hz}\right), 106.5$. IR $(\mathrm{KBr}), v\left(\mathrm{~cm}^{-1}\right) 3708,3053,1724,1519$, 1404, 1176, 858, 746; HRMS (ESI) m/z calcd for. $\mathrm{C}_{15} \mathrm{H}_{8} \mathrm{FNO}[\mathrm{M}+\mathrm{H}]^{+} 238.0663$, found 238.0662.

\section{1-(Benzo[ $h]$ furo[2,3-f]quinolin-9-yl)ethan-1-one (2g)}

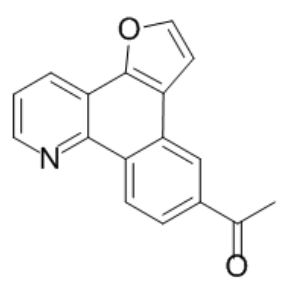

Yield: 75\% (49 mg). White solid. m.p. 134.2-135.6 ${ }^{\circ} \mathrm{C}$. Purified by flash column chromatography $($ EtOAc/hexane $=1 / 20) .{ }^{1} \mathrm{H}$ NMR $\left(400 \mathrm{MHz}, \mathrm{CDCl}_{3}\right) \delta 9.40(\mathrm{~d}, J=$ $8.6 \mathrm{~Hz}, 1 \mathrm{H}), 9.01(\mathrm{dd}, J=4.3,1.4 \mathrm{~Hz}, 1 \mathrm{H}), 8.73(\mathrm{~s}, 1 \mathrm{H}), 8.62(\mathrm{dd}, J=8.1,1.4 \mathrm{~Hz}$, $1 \mathrm{H}), 8.22(\mathrm{dd}, J=8.6,1.4 \mathrm{~Hz}, 1 \mathrm{H}), 7.90(\mathrm{~d}, J=1.8 \mathrm{~Hz}, 1 \mathrm{H}), 7.65(\mathrm{dd}, J=8.1,4.4 \mathrm{~Hz}$, $1 \mathrm{H}), 7.39(\mathrm{~d}, J=1.8 \mathrm{~Hz}, 1 \mathrm{H}), 2.81(\mathrm{~s}, 3 \mathrm{H}) .{ }^{13} \mathrm{C} \mathrm{NMR}\left(101 \mathrm{MHz}, \mathrm{CDCl}_{3}\right) \delta 198.5,148.4,148.0,145.5$, $144.8,136.7,132.5,128.5,128.2$, 126.3, 124.8, 124.2, 123.0, 121.2, 118.8, 106.7, 27.2. IR (KBr), $v$ $\left(\mathrm{cm}^{-1}\right)$ 3440, 1629, 1101, 613; HRMS (ESI) m/z calcd for. $\mathrm{C}_{17} \mathrm{H}_{11} \mathrm{NO}_{2}[\mathrm{M}+\mathrm{H}]^{+} 262.0863$, found 262.0852 .

\section{Benzo[h]furo[2,3-f]quinoline-9-carbonitrile (2h)}

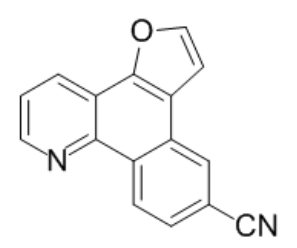

Yield: 79\% (48 mg). White solid. m.p. 239.1-239.8 ${ }^{\circ} \mathrm{C}$. Purified by flash column chromatography $($ EtOAc/hexane $=1 / 10) .{ }^{1} \mathrm{H} \mathrm{NMR}\left(600 \mathrm{MHz}, \mathrm{CDCl}_{3}\right) \delta 9.44(\mathrm{~d}, J=$ $8.5 \mathrm{~Hz}, 1 \mathrm{H}), 9.03$ (dd, $J=4.3,1.5 \mathrm{~Hz}, 1 \mathrm{H}), 8.64$ (dd, $J=8.1,1.5 \mathrm{~Hz}, 1 \mathrm{H}), 8.46$ (s, $1 \mathrm{H}), 7.92(\mathrm{~d}, J=1.9 \mathrm{~Hz}, 1 \mathrm{H}), 7.88(\mathrm{dd}, J=8.5,1.4 \mathrm{~Hz}, 1 \mathrm{H}), 7.69(\mathrm{dd}, J=8.1,4.4$

$\mathrm{Hz}, 1 \mathrm{H}), 7.31(\mathrm{~d}, J=1.9 \mathrm{~Hz}, 1 \mathrm{H}) .{ }^{13} \mathrm{C} \mathrm{NMR}\left(151 \mathrm{MHz}, \mathrm{CDCl}_{3}\right) \delta 148.7,148.4,145.8,144.4,132.0$, 128.6, 128.4, 128.2, 127.5, 127.0, 123.4, 120.1, 119.3, 118.9, 112.1, 106.5. IR (KBr), v (cm $\left.{ }^{-1}\right) 3444$, 2852, 2223, 1629, 1099, 763; HRMS (ESI) m/z calcd for. $\mathrm{C}_{16} \mathrm{H}_{8} \mathrm{~N}_{2} \mathrm{O}[\mathrm{M}+\mathrm{Na}]^{+} 267.0529$, found 267.0525 . 


\section{Benzo $[h]$ benzofuro[2,3- $f]$ quinoline-7-carbonitrile (2i)}

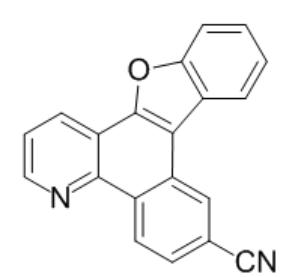

Yield: $78 \%$ (57 mg). White solid. m.p. 262.8-263.9 ${ }^{\circ} \mathrm{C}$. Purified by flash column chromatography $($ EtOAc/hexane $=1 / 10) .{ }^{1} \mathrm{H}$ NMR $\left(400 \mathrm{MHz}, \mathrm{CDCl}_{3}\right) \delta 9.46(\mathrm{~d}, J=$ $8.5 \mathrm{~Hz}, 1 \mathrm{H}), 9.07$ (dd, $J=4.4,1.7 \mathrm{~Hz}, 1 \mathrm{H}), 8.79$ (d, $J=1.2 \mathrm{~Hz}, 1 \mathrm{H}), 8.72$ (dd, $J=8.1$, $1.7 \mathrm{~Hz}, 1 \mathrm{H}), 8.29(\mathrm{dd}, J=6.4,2.5 \mathrm{~Hz}, 1 \mathrm{H}), 7.88(\mathrm{dd}, J=8.5,1.5 \mathrm{~Hz}, 1 \mathrm{H}), 7.76(\mathrm{dd}, J$ $=6.8,2.3 \mathrm{~Hz}, 1 \mathrm{H}), 7.71(\mathrm{dd}, J=8.1,4.4 \mathrm{~Hz}, 1 \mathrm{H}), 7.56(\mathrm{pd}, J=7.3,3.7 \mathrm{~Hz}, 2 \mathrm{H}) \cdot{ }^{13} \mathrm{C} \mathrm{NMR}(101 \mathrm{MHz}$, $\left.\mathrm{CDCl}_{3}\right) \delta 157.5,156.4,150.3,149.7,145.4,131.8,129.5,129.0,128.3,127.2,127.1,126.8,124.4$, 124.3, 123.5, 121.8, 119.4, 118.5, 114.4, 112.5. IR (KBr), $v\left(\mathrm{~cm}^{-1}\right) 3703,3055,1737,1450,960$; HRMS (ESI) $\mathrm{m} / \mathrm{z}$ calcd for. $\mathrm{C}_{20} \mathrm{H}_{10} \mathrm{~N}_{2} \mathrm{O}[\mathrm{M}+\mathrm{H}]^{+}$295.0866, found 295.0871 .

\section{Benzo $[h]$ thieno[2,3- $f$ ]quinoline-9-carbonitrile (2j)}

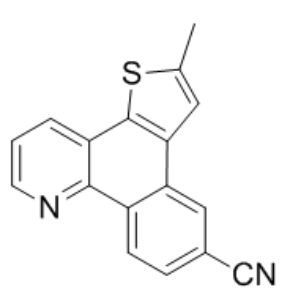

Yield: $80 \%$ (54 mg). Yellow solid. m.p. 229.9-231.5 ${ }^{\circ} \mathrm{C}$. Purified by flash column chromatography $($ EtOAc/hexane $=1 / 10) .{ }^{1} \mathrm{H}$ NMR $\left(400 \mathrm{MHz}, \mathrm{CDCl}_{3}\right) \delta 9.30(\mathrm{~d}, J$ $=8.5 \mathrm{~Hz}, 1 \mathrm{H}), 8.92(\mathrm{~d}, J=3.1 \mathrm{~Hz}, 1 \mathrm{H}), 8.39(\mathrm{~s}, 1 \mathrm{H}), 8.24(\mathrm{~d}, J=8.0 \mathrm{~Hz}, 1 \mathrm{H}), 7.79$ $(\mathrm{d}, J=8.4 \mathrm{~Hz}, 1 \mathrm{H}), 7.55(\mathrm{dd}, J=7.8,4.3 \mathrm{~Hz}, 1 \mathrm{H}), 7.49(\mathrm{~s}, 1 \mathrm{H}), 2.71(\mathrm{~s}, 3 \mathrm{H}) .{ }^{13} \mathrm{C}$ NMR (101 MHz, $\left.\mathrm{CDCl}_{3}\right) \delta 148.4,143.4,142.4,135.0,134.5,132.6,131.4,129.3$, 128.6, 127.7, 126.7, 124.5, 123.4, 120.9, 119.4, 111.8, 16.4. IR (KBr), v (cm $\left.{ }^{-1}\right) 3427,2223,1631,1076$, 607; HRMS (ESI) m/z calcd for. $\mathrm{C}_{17} \mathrm{H}_{10} \mathrm{~N}_{2} \mathrm{~S}[\mathrm{M}+\mathrm{Na}]^{+} 297.0457$, found 297.0461.

\section{9-(Trifluoromethyl)benzo[ $h]$ furo[2,3- $f]$ quinoline (2k)}

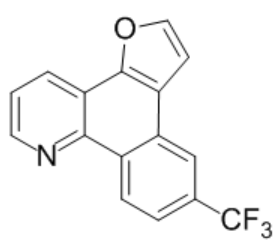

Yield: $72 \%$ (51 mg). White solid. m.p. 98.6-99.8 ${ }^{\circ} \mathrm{C}$. Purified by flash column chromatography $($ EtOAc/hexane $=1 / 20) .{ }^{1} \mathrm{H} \mathrm{NMR}\left(600 \mathrm{MHz}, \mathrm{CDCl}_{3}\right) \delta 9.40(\mathrm{~d}, J=$ $8.6 \mathrm{~Hz}, 1 \mathrm{H}), 8.99(\mathrm{dd}, J=4.3,1.6 \mathrm{~Hz}, 1 \mathrm{H}), 8.58(\mathrm{dd}, J=8.1,1.6 \mathrm{~Hz}, 1 \mathrm{H}), 8.34$ (s, $1 \mathrm{H}), 7.89-7.84(\mathrm{~m}, 2 \mathrm{H}), 7.63(\mathrm{dd}, J=8.1,4.4 \mathrm{~Hz}, 1 \mathrm{H}), 7.29(\mathrm{~d}, J=2.0 \mathrm{~Hz}, 1 \mathrm{H})$. ${ }^{13} \mathrm{C} \mathrm{NMR}\left(151 \mathrm{MHz}, \mathrm{CDCl}_{3}\right) \delta 148.4,148.2,145.4,144.6,131.5,130.4\left(\mathrm{q},{ }^{2} J=31.5 \mathrm{~Hz}\right), 128.4,128.0$, 126.8, $124.6\left(\mathrm{q},{ }^{1} J=270 \mathrm{~Hz}\right), 122.9,121.7\left(\mathrm{q},{ }^{3} J=3 \mathrm{~Hz}\right), 120.8\left(\mathrm{q},{ }^{3} J=3 \mathrm{~Hz}\right), 120.6,118.4$, 106.5. IR $(\mathrm{KBr}), v\left(\mathrm{~cm}^{-1}\right)$ 3450, 2962, 1583, 1099, 800; HRMS (ESI) $\mathrm{m} / \mathrm{z}$ calcd for. $\mathrm{C}_{16} \mathrm{H}_{8} \mathrm{~F}_{3} \mathrm{NO}[\mathrm{M}+\mathrm{H}]^{+}$ 288.0631, found 288.0633.

\section{6-Methyl-9-(trifluoromethyl)benzo[ $h]$ thieno[2,3-f]quinoline (2l)}

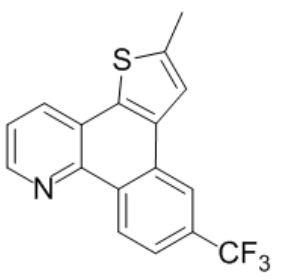

Yield: $80 \%$ (63 mg). Yellow solid. m.p. 110.5-112.5 ${ }^{\circ} \mathrm{C}$. Purified by flash column chromatography $($ EtOAc/hexane $=1 / 20) .{ }^{1} \mathrm{H} \mathrm{NMR}\left(400 \mathrm{MHz}, \mathrm{CDCl}_{3}\right) \delta 9.31(\mathrm{~d}, J=$ $8.6 \mathrm{~Hz}, 1 \mathrm{H}), 8.89$ (dd, $J=4.3,1.4 \mathrm{~Hz}, 1 \mathrm{H}), 8.34$ (s, 1H), 8.17 (dd, $J=8.1,1.4 \mathrm{~Hz}$,

$1 \mathrm{H}), 7.82(\mathrm{~d}, J=8.6 \mathrm{~Hz}, 1 \mathrm{H}), 7.49(\mathrm{dd}, J=8.2,4.4 \mathrm{~Hz}, 2 \mathrm{H}), 2.67(\mathrm{~s}, 3 \mathrm{H}) .{ }^{13} \mathrm{C} \mathrm{NMR}$ $\left(101 \mathrm{MHz}, \mathrm{CDCl}_{3}\right) \delta 148.2,143.8,141.8,135.3,134.6,132.2,131.3,130.1\left(\mathrm{q},{ }^{2} J=32 \mathrm{~Hz}\right), 129.2,126.8$, 126.5, $124.0\left(\mathrm{q},{ }^{1} J=271 \mathrm{~Hz}\right), 122.9,122.0\left(\mathrm{q},{ }^{3} J=3 \mathrm{~Hz}\right), 121.0,121.0\left(\mathrm{q},{ }^{3} J=4 \mathrm{~Hz}\right), 16.3$. IR (KBr), $v$ $\left(\mathrm{cm}^{-1}\right)$ 2920, 1531, 1317, 1112, 783; HRMS (ESI) m/z calcd for. $\mathrm{C}_{17} \mathrm{H}_{10} \mathrm{~F}_{3} \mathrm{NS}[\mathrm{M}+\mathrm{H}]^{+} 318.0559$, found 318.0553. 


\section{Benzo[h]furo[2,3-f]quinoline-10-carbonitrile (2m)}

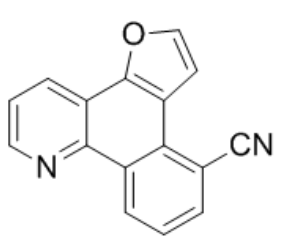

Yield: $82 \%$ (50 mg). White solid. m.p. 195.1-196.5 ${ }^{\circ} \mathrm{C}$. Purified by flash column chromatography $($ EtOAc/hexane $=1 / 10) .{ }^{1} \mathrm{H} \mathrm{NMR}\left(400 \mathrm{MHz}, \mathrm{CDCl}_{3}\right) \delta 9.63(\mathrm{~d}, J=$ $8.3 \mathrm{~Hz}, 1 \mathrm{H}), 9.05-8.95(\mathrm{~m}, 1 \mathrm{H}), 8.63(\mathrm{~d}, J=8.1 \mathrm{~Hz}, 1 \mathrm{H}), 8.09$ (t, $J=4.3 \mathrm{~Hz}, 2 \mathrm{H})$,

$7.90(\mathrm{~d}, J=2.0 \mathrm{~Hz}, 1 \mathrm{H}), 7.72(\mathrm{t}, J=7.9 \mathrm{~Hz}, 1 \mathrm{H}), 7.66(\mathrm{dd}, J=8.1,4.4 \mathrm{~Hz}, 1 \mathrm{H}) .{ }^{13} \mathrm{C}$ NMR $\left(101 \mathrm{MHz}, \mathrm{CDCl}_{3}\right) \delta 149.3,148.8,145.1,144.4,135.4,131.0,130.0,128.7,128.6,125.0,123.2$, 120.1, 118.6, 117.8, 108.2, 107.2. IR (KBr), $v\left(\mathrm{~cm}^{-1}\right)$ 3718, 2962, 2218, 1753, 1519, 1261, 1109, 804; HRMS (ESI) m/z calcd for. $\mathrm{C}_{16} \mathrm{H}_{8} \mathrm{~N}_{2} \mathrm{O}[\mathrm{M}+\mathrm{H}]^{+} 245.0709$, found 245.0715.

\section{1-(Benzo[h]furo[2,3-f]quinolin-10-yl)ethan-1-one (2n)}

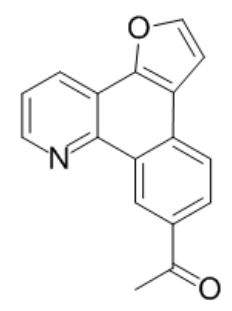

Yield: 79\% (51 mg). Yellow solid. m.p. 98.4-99.8 ${ }^{\circ} \mathrm{C}$. Purified by flash column chromatography $($ EtOAc/hexane $=1 / 20) .{ }^{1} \mathrm{H}$ NMR $\left(400 \mathrm{MHz}, \mathrm{CDCl}_{3}\right) \delta 9.93(\mathrm{~s}, 1 \mathrm{H})$, $9.02(\mathrm{dd}, J=4.3,1.1 \mathrm{~Hz}, 1 \mathrm{H}), 8.62(\mathrm{dd}, J=8.1,1.2 \mathrm{~Hz}, 1 \mathrm{H}), 8.35(\mathrm{dd}, J=8.4,1.3 \mathrm{~Hz}$, $1 \mathrm{H}), 8.17(\mathrm{~d}, J=8.4 \mathrm{~Hz}, 1 \mathrm{H}), 7.89(\mathrm{~d}, J=1.7 \mathrm{~Hz}, 1 \mathrm{H}), 7.64(\mathrm{dd}, J=8.1,4.4 \mathrm{~Hz}, 1 \mathrm{H})$, $7.32(\mathrm{~d}, J=1.7 \mathrm{~Hz}, 1 \mathrm{H}), 2.87(\mathrm{~s}, 3 \mathrm{H}) .{ }^{13} \mathrm{C} \mathrm{NMR}\left(101 \mathrm{MHz}, \mathrm{CDCl}_{3}\right) \delta$ 198.5, 149.1, 148.4, 145.5, 145.5, 134.4, 131.6, 128.8, 128.6, 127.6, 127.3, 124.0, 122.6, 120.5, 117.9, 106.7, 27.0. IR $(\mathrm{KBr}), v\left(\mathrm{~cm}^{-1}\right)$ 3708, 2923, 1689, 1515, 1259, 966, 754; HRMS (ESI) m/z calcd for. $\mathrm{C}_{17} \mathrm{H}_{11} \mathrm{NO}_{2}$ $[\mathrm{M}+\mathrm{H}]^{+}$262.0863, found 262.0860.

\section{Furo[2,3-f]naphtho[1,2-h]quinoline (2o)}

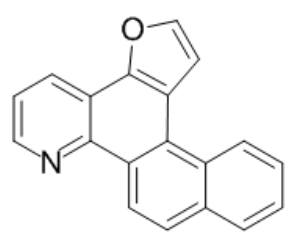

Yield: 59\% (40 mg). Yellow solid. m.p. 107.2-108.5 ${ }^{\circ} \mathrm{C}$. Purified by flash column chromatography $($ EtOAc/hexane $=1 / 20) .{ }^{1} \mathrm{H}$ NMR $\left(400 \mathrm{MHz}, \mathrm{CDCl}_{3}\right) \delta 9.50(\mathrm{~d}, J$ $=9.0 \mathrm{~Hz}, 1 \mathrm{H}), 9.10-9.08(\mathrm{~m}, 1 \mathrm{H}), 9.07(\mathrm{~d}, J=1.8 \mathrm{~Hz}, 1 \mathrm{H}), 8.72(\mathrm{dd}, J=8.1,1.8$ $\mathrm{Hz}, 1 \mathrm{H}), 8.07(\mathrm{t}, J=7.3 \mathrm{~Hz}, 2 \mathrm{H}), 8.01(\mathrm{~d}, J=2.2 \mathrm{~Hz}, 1 \mathrm{H}), 7.91(\mathrm{~d}, J=2.2 \mathrm{~Hz}, 1 \mathrm{H})$, $7.78-7.73(\mathrm{~m}, 1 \mathrm{H}), 7.71-7.67(\mathrm{~m}, 1 \mathrm{H}), 7.64(\mathrm{dd}, J=8.1,4.3 \mathrm{~Hz}, 1 \mathrm{H}) .{ }^{13} \mathrm{C} \mathrm{NMR}\left(101 \mathrm{MHz}, \mathrm{CDCl}_{3}\right) \delta$ $149.1,148.6,145.0,144.9,134.1,130.7,129.1,128.5,128.0,126.8,126.5,126.5,126.0,125.8,123.5$, 122.0, 119.7, 117.0, 109.9. IR (KBr), $v\left(\mathrm{~cm}^{-1}\right) 3714,2923,1728,1575,1317,1085,763$; HRMS (ESI) $\mathrm{m} / \mathrm{z}$ calcd for. $\mathrm{C}_{19} \mathrm{H}_{11} \mathrm{NO}[\mathrm{M}+\mathrm{H}]^{+} 270.0913$, found 270.0910 .

\section{3-Methylbenzo[h]furo[2,3-f]quinoline-9-carbonitrile (2p)}

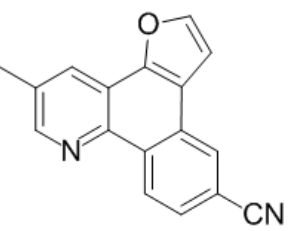

Yield: 77\% (49 mg). White solid. m.p. 237.3-238.9 ${ }^{\circ} \mathrm{C}$. Purified by flash column chromatography $($ EtOAc/hexane $=1 / 10) .{ }^{1} \mathrm{H}$ NMR $\left(400 \mathrm{MHz}, \mathrm{CDCl}_{3}\right) \delta 9.34(\mathrm{~d}, J=$ $8.5 \mathrm{~Hz}, 1 \mathrm{H}), 8.84(\mathrm{~d}, J=2.1 \mathrm{~Hz}, 1 \mathrm{H}), 8.52-8.31(\mathrm{~m}, 2 \mathrm{H}), 7.88(\mathrm{~d}, J=2.0 \mathrm{~Hz}, 1 \mathrm{H})$, $7.83(\mathrm{dd}, J=8.5,1.5 \mathrm{~Hz}, 1 \mathrm{H}), 7.27(\mathrm{~d}, J=2.0 \mathrm{~Hz}, 1 \mathrm{H}), 2.63(\mathrm{~s}, 3 \mathrm{H}) .{ }^{13} \mathrm{C}$ NMR $(101$ $\left.\mathrm{MHz}, \mathrm{CDCl}_{3}\right) \delta 150.2,148.3,145.6,142.3,133.5,132.0,128.4,127.8,127.7,127.3,126.6,120.0,119.4$, 118.5, 111.5, 106.4, 19.1. IR (KBr), $v\left(\mathrm{~cm}^{-1}\right)$ 3454, 3097, 2283, 2216, 1566, 1323, 1143, 771, 673; HRMS (ESI) $\mathrm{m} / \mathrm{z}$ calcd for. $\mathrm{C}_{17} \mathrm{H}_{10} \mathrm{~N}_{2} \mathrm{O}[\mathrm{M}+\mathrm{Na}]^{+} 281.0685$, found 281.0680 .

\section{3,6-Dimethylbenzo[h]thieno[2,3-f]quinoline-9-carbonitrile (2q)}




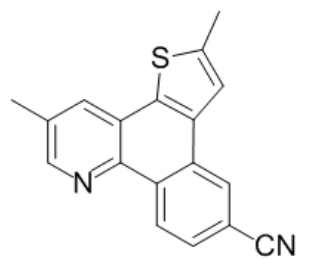

Yield: $79 \%$ (56 mg). White solid. m.p. 217.9-219.8 ${ }^{\circ} \mathrm{C}$. Purified by flash column chromatography $($ EtOAc/hexane $=1 / 10) .{ }^{1} \mathrm{H} \mathrm{NMR}\left(400 \mathrm{MHz}, \mathrm{CDCl}_{3}\right) \delta 9.27(\mathrm{~d}, J=$ $8.5 \mathrm{~Hz}, 1 \mathrm{H}), 8.76(\mathrm{~d}, J=1.8 \mathrm{~Hz}, 1 \mathrm{H}), 8.42(\mathrm{~s}, 1 \mathrm{H}), 8.02$ (s, 1H), 7.79 (dd, $J=8.5$,

$1.3 \mathrm{~Hz}, 1 \mathrm{H}), 7.52(\mathrm{~s}, 1 \mathrm{H}), 2.72(\mathrm{~s}, 3 \mathrm{H}), 2.59(\mathrm{~s}, 3 \mathrm{H}) .{ }^{13} \mathrm{C} \mathrm{NMR}\left(101 \mathrm{MHz}, \mathrm{CDCl}_{3}\right) \delta$ 150.0, 142.1, 141.3, 134.9, 134.6, 133.4, 132.7, 130.8, 128.9, 128.7, 127.7, 126.4, 124.2, 121.0, 119.5, 111.3, 19.0, 16.4. IR (KBr), $v\left(\mathrm{~cm}^{-1}\right) 3450,2918,2221,1604,1132,840,599 ;$ HRMS (ESI) m/z calcd for. $\mathrm{C}_{18} \mathrm{H}_{12} \mathrm{~N}_{2} \mathrm{~S}[\mathrm{M}+\mathrm{H}]^{+} 289.0794$, found 289.0790.

\section{1-(3,6-Dimethylbenzo[ $h]$ furo[2,3-f]quinolin-9-yl)ethan-1-one (2r)}

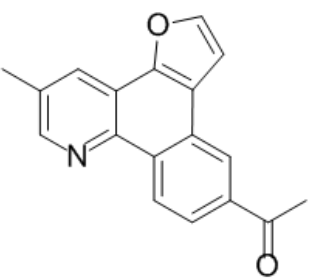

Yield: $71 \%$ (51 mg). Yellow solid. m.p. 192.8-194.0 ${ }^{\circ} \mathrm{C}$. Purified by flash column chromatography $($ EtOAc/hexane $=1 / 20) .{ }^{1} \mathrm{H}$ NMR $\left(400 \mathrm{MHz}, \mathrm{CDCl}_{3}\right) \delta 9.15(\mathrm{~d}, J$ $=8.5 \mathrm{~Hz}, 1 \mathrm{H}), 8.68(\mathrm{~s}, 1 \mathrm{H}), 8.51(\mathrm{~s}, 1 \mathrm{H}), 8.19(\mathrm{~s}, 1 \mathrm{H}), 8.04(\mathrm{~d}, J=8.4 \mathrm{~Hz}, 1 \mathrm{H})$, $7.71(\mathrm{~d}, J=15.3 \mathrm{~Hz}, 1 \mathrm{H}), 7.19(\mathrm{~s}, 1 \mathrm{H}), 2.68(\mathrm{~s}, 3 \mathrm{H}), 2.50(\mathrm{~s}, 3 \mathrm{H}) .{ }^{13} \mathrm{C}$ NMR $(101$ $\left.\mathrm{MHz}, \mathrm{CDCl}_{3}\right) \delta 198.3,149.7,147.8,145.1,142.6,136.1,132.8,132.4,127.6,127.5,125.8,124.5,124.0$, 121.1, 118.3, 106.6, 29.8, 27.1. IR (KBr), $v\left(\mathrm{~cm}^{-1}\right)$ 3749, 2923, 1743, 1672, 1350, 1244, 1016, 773; HRMS (ESI) m/z calcd for. $\mathrm{C}_{18} \mathrm{H}_{13} \mathrm{NO}_{2}[\mathrm{M}+\mathrm{H}]^{+} 276.1019$, found 276.1014.

\section{trans-8b,13a-Dihydrobenzo[ $f]$ benzofuro $[3,2-h]$ quinoline $(2 s)$}

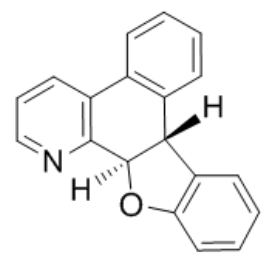

Yield: 43\% (29 mg). White solid. m.p. 159.4-161.4 ${ }^{\circ} \mathrm{C}$. Purified by flash column chromatography $($ EtOAc/hexane $=1 / 30) .{ }^{1} \mathrm{H}$ NMR $\left(400 \mathrm{MHz}, \mathrm{CDCl}_{3}\right) \delta 8.59(\mathrm{~d}, J=$ $4.7 \mathrm{~Hz}, 1 \mathrm{H}), 8.03$ (d, $J=7.9 \mathrm{~Hz}, 1 \mathrm{H}), 7.87$ (d, $J=2.6 \mathrm{~Hz}, 1 \mathrm{H}), 7.72$ (dd, $J=15.6,5.2$ $\mathrm{Hz}, 2 \mathrm{H}), 7.39$ (ddd, $J=12.4,6.1,3.9 \mathrm{~Hz}, 3 \mathrm{H}), 7.28-7.23(\mathrm{~m}, 1 \mathrm{H}), 7.14$ (d, $J=8.0 \mathrm{~Hz}$, $1 \mathrm{H}), 7.07(\mathrm{t}, J=7.4 \mathrm{~Hz}, 1 \mathrm{H}), 5.26(\mathrm{~d}, J=16.3 \mathrm{~Hz}, 1 \mathrm{H}), 4.58(\mathrm{~d}, J=16.3 \mathrm{~Hz}, 1 \mathrm{H}) .{ }^{13} \mathrm{C} \mathrm{NMR}(101 \mathrm{MHz}$, $\left.\mathrm{CDCl}_{3}\right) \delta 161.4,155.6,148.4,135.5,133.1,132.0,129.1,129.0,128.7,127.9,127.5,125.4,124.6$, 124.2, 123.3, 121.7, 111.8, 85.9, 48.3. IR (KBr), $v\left(\mathrm{~cm}^{-1}\right) 3446,1637,1097,748 ;$ HRMS (ESI) m/z calcd for. $\mathrm{C}_{19} \mathrm{H}_{13} \mathrm{NO}[\mathrm{M}+\mathrm{H}]^{+} 272.1070$, found 272.1066 .

\section{cis-8b,13a-Dihydrobenzo[f]benzofuro[3,2- $h]$ quinoline $(2 t)$}

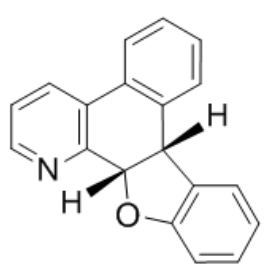

Yield: 39\% (26 mg). White solid. m.p. 168.1-169.4 ${ }^{\circ} \mathrm{C}$. Purified by flash column chromatography $($ EtOAc/hexane $=1 / 30) .{ }^{1} \mathrm{H}$ NMR $\left(400 \mathrm{MHz}, \mathrm{CDCl}_{3}\right) \delta 8.54(\mathrm{~d}, J=$ $4.3 \mathrm{~Hz}, 1 \mathrm{H}), 7.99$ (d, $J=7.8 \mathrm{~Hz}, 1 \mathrm{H}), 7.69$ (d, $J=7.4 \mathrm{~Hz}, 1 \mathrm{H}), 7.43$ (d, $J=7.2 \mathrm{~Hz}$, $1 \mathrm{H}), 7.30-7.15(\mathrm{~m}, 4 \mathrm{H}), 7.03(\mathrm{t}, J=7.6 \mathrm{~Hz}, 1 \mathrm{H}), 6.93-6.65(\mathrm{~m}, 2 \mathrm{H}), 5.91(\mathrm{~d}, J=$ $9.1 \mathrm{~Hz}, 1 \mathrm{H}), 4.78(\mathrm{~d}, J=9.0 \mathrm{~Hz}, 1 \mathrm{H}) .{ }^{13} \mathrm{C} \mathrm{NMR}\left(101 \mathrm{MHz}, \mathrm{CDCl}_{3}\right) \delta 158.7,150.5,149.5,132.7,130.5$, 130.0, 129.5, 129.5, 129.2, 128.7, 128.4, 127.9, 124.9, 124.2, 123.6, 121.1, 110.3, 82.3, 44.7. IR (KBr), $v\left(\mathrm{~cm}^{-1}\right)$ 3070, 1755, 1454, 1211, 964, 750, 597; HRMS (ESI) m/z calcd for. $\mathrm{C}_{19} \mathrm{H}_{13} \mathrm{NO}[\mathrm{M}+\mathrm{H}]^{+}$ 272.1070, found 272.1069 .

cis-Methyl-8b,13a-dihydrobenzo[f]benzofuro[3,2-h]quinolone (2u) 


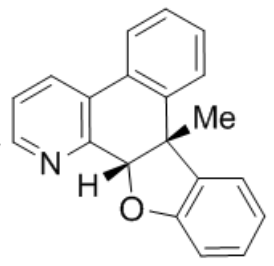

Yield: $77 \%$ (55 mg). White solid. m.p. 148.2-149.3 ${ }^{\circ} \mathrm{C}$. Purified by flash column chromatography $($ EtOAc/hexane $=1 / 30) .{ }^{1} \mathrm{H}$ NMR $\left(400 \mathrm{MHz}, \mathrm{CDCl}_{3}\right) \delta 8.68(\mathrm{dd}, J=$ 4.7, $1.3 \mathrm{~Hz}, 1 \mathrm{H}), 8.25(\mathrm{~d}, J=8.1 \mathrm{~Hz}, 1 \mathrm{H}), 7.86-7.81(\mathrm{~m}, 1 \mathrm{H}), 7.62(\mathrm{~d}, J=7.4 \mathrm{~Hz}$,

$1 \mathrm{H}), 7.55-7.50(\mathrm{~m}, 1 \mathrm{H}), 7.45(\mathrm{dd}, J=8.0,4.7 \mathrm{~Hz}, 1 \mathrm{H}), 7.30(\mathrm{pd}, J=7.3,3.6 \mathrm{~Hz}$, 2H), $7.18(\mathrm{dd}, J=11.0,4.4 \mathrm{~Hz}, 1 \mathrm{H}), 7.05(\mathrm{t}, J=7.4 \mathrm{~Hz}, 1 \mathrm{H}), 6.86(\mathrm{~d}, J=7.9 \mathrm{~Hz}, 1 \mathrm{H}), 5.41$ (s, 1H), 1.67 $(\mathrm{s}, 3 \mathrm{H}) .{ }^{13} \mathrm{C}$ NMR $\left(101 \mathrm{MHz}, \mathrm{CDCl}_{3}\right) \delta 159.6,149.5,148.8,138.8,134.8,131.1,129.6,129.2,128.7$, 128.5, 128.4, 127.5, 125.2, 124.4, 123.6, 121.4, 110.4, 90.0, 48.1, 25.7. IR (KBr), v $\left(\mathrm{cm}^{-1}\right) 3452,2923$, 1461, 1220 ,752; $\mathrm{C}_{20} \mathrm{H}_{15} \mathrm{NO}[\mathrm{M}+\mathrm{H}]^{+}$286.1226, found 286.1228.

\section{8b-Methyl-1,8b-dihydrobenzo[ $f]$ benzofuro $[3,2-h]$ quinoline $(2 \mathrm{v})$}

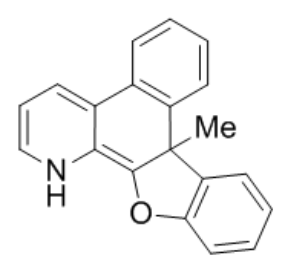

Yield: $18 \%(13 \mathrm{mg})$. Colorless oil. Purified by flash column chromatography $($ EtOAc/hexane $=1 / 20) .{ }^{1} \mathrm{H}$ NMR $(400 \mathrm{MHz}, \mathrm{DMSO}) \delta 9.17(\mathrm{dd}, J=8.4,1.4 \mathrm{~Hz}, 1 \mathrm{H})$, $8.92(\mathrm{~s}, 1 \mathrm{H}), 8.87(\mathrm{dd}, J=6.4,3.1 \mathrm{~Hz}, 1 \mathrm{H}), 8.72(\mathrm{dd}, J=4.2,1.3 \mathrm{~Hz}, 1 \mathrm{H}), 8.16(\mathrm{dd}, J$ $=6.3,3.2 \mathrm{~Hz}, 1 \mathrm{H}), 7.76-7.68(\mathrm{~m}, 2 \mathrm{H}), 7.54(\mathrm{dd}, J=8.3,4.3 \mathrm{~Hz}, 1 \mathrm{H}), 7.23-7.15(\mathrm{~m}$, $1 \mathrm{H}), 6.98(\mathrm{dd}, J=7.4,1.4 \mathrm{~Hz}, 1 \mathrm{H}), 6.91(\mathrm{~d}, J=8.0 \mathrm{~Hz}, 1 \mathrm{H}), 6.85(\mathrm{t}, J=7.3 \mathrm{~Hz}, 1 \mathrm{H}), 2.41(\mathrm{~s}, 3 \mathrm{H}) .{ }^{13} \mathrm{C}$ NMR (101 MHz, DMSO) $\delta 154.9,149.1,147.2$, 135.4, 134.5, 131.7, 131.2, 130.7, 129.0, 128.0, 127.6, $126.8,126.8,125.1,123.9,123.3,120.8,118.5,115.3,17.1$. IR (KBr), $v\left(\mathrm{~cm}^{-1}\right) 3417,2252,1450$, 1026,$761 ; \mathrm{C}_{20} \mathrm{H}_{15} \mathrm{NO}[\mathrm{M}+\mathrm{H}]^{+} 286.1226$, found 286.1229.

\section{Benzo[f]furo[3,2- $h]$ isoquinoline (5a)}

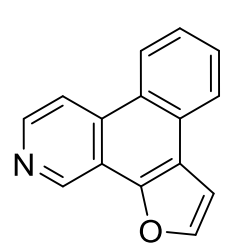

Yield: $56 \%$ (31mg). White solid. m.p. $126.8-127.9^{\circ} \mathrm{C}$. Purified by flash column chromatography $($ EtOAc/hexane $=1 / 20) .{ }^{1} \mathrm{H}$ NMR $\left(400 \mathrm{MHz}, \mathrm{CDCl}_{3}\right) \delta 9.70(\mathrm{~s}, 1 \mathrm{H})$, $8.78(\mathrm{~d}, J=5.8 \mathrm{~Hz}, 1 \mathrm{H}), 8.70(\mathrm{~d}, J=8.3 \mathrm{~Hz}, 1 \mathrm{H}), 8.44(\mathrm{~d}, J=5.8 \mathrm{~Hz}, 1 \mathrm{H}), 8.17(\mathrm{~d}, J=$ $8.0 \mathrm{~Hz}, 1 \mathrm{H}), 7.87(\mathrm{~d}, J=2.1 \mathrm{~Hz}, 1 \mathrm{H}), 7.77(\mathrm{t}, J=7.5 \mathrm{~Hz}, 1 \mathrm{H}), 7.67(\mathrm{t}, J=7.7 \mathrm{~Hz}, 1 \mathrm{H})$, 7.34 - $7.21(\mathrm{~m}, 2 \mathrm{H}) .{ }^{13} \mathrm{C}$ NMR $\left(101 \mathrm{MHz}, \mathrm{CDCl}_{3}\right) \delta 145.0,144.9,144.8,129.3,129.3,126.4,125.9$, 124.3, 121.8, 117.9, 116.9, 106.5; IR (KBr), $v\left(\mathrm{~cm}^{-1}\right) 3448,1629,1346,1139,744$; HRMS (ESI) m/z calcd for. $\mathrm{C}_{15} \mathrm{H}_{9} \mathrm{NO}[\mathrm{M}+\mathrm{H}]^{+} 220.0757$, found 220.0753 .

\section{5-Methylbenzo[f]furo[3,2- $h]$ isoquinoline $(5 b)$}

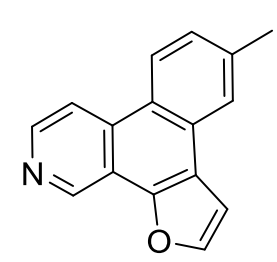

Yield: $60 \%$ (35mg). White solid. m.p. $117-118{ }^{\circ} \mathrm{C}$. Purified by flash column chromatography $($ EtOAc/hexane $=1 / 20) .{ }^{1} \mathrm{H} \mathrm{NMR}\left(400 \mathrm{MHz}, \mathrm{CDCl}_{3}\right) \delta 9.58(\mathrm{~s}, 1 \mathrm{H})$, $8.69(\mathrm{~d}, J=5.8 \mathrm{~Hz}, 1 \mathrm{H}), 8.42(\mathrm{~d}, J=8.4 \mathrm{~Hz}, 1 \mathrm{H}), 8.26(\mathrm{~d}, J=5.7 \mathrm{~Hz}, 1 \mathrm{H}), 7.86-$ $7.72(\mathrm{~m}, 2 \mathrm{H}), 7.40(\mathrm{~d}, J=8.4 \mathrm{~Hz}, 1 \mathrm{H}), 7.17(\mathrm{~d}, J=1.9 \mathrm{~Hz}, 1 \mathrm{H}), 2.58(\mathrm{~s}, 3 \mathrm{H}) .{ }^{13} \mathrm{C}$ NMR $\left(101 \mathrm{MHz}, \mathrm{CDCl}_{3}\right) \delta 147.5,144.7,144.6,144.4,139.4,133.6,129.2,127.4,124.0,124.0,123.9$, 121.4, 117.4, 116.6, 106.3, 21.9; IR (KBr), $v\left(\mathrm{~cm}^{-1}\right) 3450,1625,1344,1141,740,553$; HRMS (ESI) $\mathrm{m} / \mathrm{z}$ calcd for. $\mathrm{C}_{16} \mathrm{H}_{11} \mathrm{NO}[\mathrm{M}+\mathrm{H}]^{+} 234.0913$, found 234.0909 .

1-(Benzo[f]furo[3,2- $h]$ isoquinolin-5-yl)ethan-1-one (5c) 


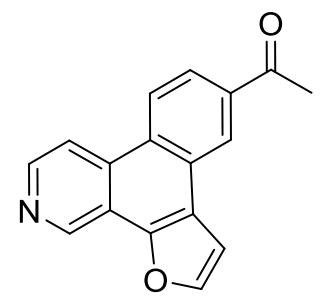

Yield: $43 \%$ (28mg). Yellow solid. $156.3-157.8^{\circ} \mathrm{C}$. Purified by flash column chromatography $($ EtOAc/hexane $=1 / 20) .{ }^{1} \mathrm{H}$ NMR $\left(400 \mathrm{MHz}, \mathrm{CDCl}_{3}\right) \delta 9.68(\mathrm{~s}$, $1 \mathrm{H}), 8.80(\mathrm{~d}, J=5.7 \mathrm{~Hz}, 1 \mathrm{H}), 8.67(\mathrm{~d}, J=8.9 \mathrm{~Hz}, 2 \mathrm{H}), 8.38(\mathrm{~d}, J=5.8 \mathrm{~Hz}, 1 \mathrm{H})$, $8.15(\mathrm{dd}, J=8.6,1.9 \mathrm{~Hz}, 1 \mathrm{H}), 7.88(\mathrm{~d}, J=2.1 \mathrm{~Hz}, 1 \mathrm{H}), 7.33(\mathrm{~d}, J=2.1 \mathrm{~Hz}, 1 \mathrm{H})$, $2.79(\mathrm{~s}, 3 \mathrm{H}) .{ }^{13} \mathrm{C} \mathrm{NMR}\left(101 \mathrm{MHz}, \mathrm{CDCl}_{3}\right) \delta 197.9,145.4,145.3,144.9,136.9$, 132.8, 129.4, 128.9, 124.8, 124.7, 124.7, 122.1, 118.3, 117.2, 106.5, 27.1; IR (KBr), v ( $\mathrm{cm}^{-1}$ ) 3442, 1672, 1080, 750, 607; HRMS (ESI) m/z calcd for. $\mathrm{C}_{17} \mathrm{H}_{11} \mathrm{NO}_{2}[\mathrm{M}+\mathrm{H}]^{+}$262.0863, found 262.0854.

\section{2-Methylbenzo[ $f]$ thieno[3,2- $h]$ isoquinoline $(5 \mathrm{~d})$}<smiles>Cc1cc2c3ccccc3c3ccncc3c2s1</smiles>

Yield: $66 \%$ (27mg). Yellow solid. m.p. $126.8-127.9^{\circ} \mathrm{C}$. Purified by flash column chromatography $($ EtOAc/hexane $=1 / 20) .{ }^{1} \mathrm{H}$ NMR $\left(400 \mathrm{MHz}, \mathrm{CDCl}_{3}\right) \delta 9.30(\mathrm{~s}, 1 \mathrm{H})$, $8.64(\mathrm{~d}, J=5.5 \mathrm{~Hz}, 1 \mathrm{H}), 8.57(\mathrm{~d}, J=8.2 \mathrm{~Hz}, 1 \mathrm{H}), 8.30(\mathrm{~d}, J=5.7 \mathrm{~Hz}, 1 \mathrm{H}), 8.18(\mathrm{~d}, J=$

$8.0 \mathrm{~Hz}, 1 \mathrm{H}), 7.70(\mathrm{t}, J=7.5 \mathrm{~Hz}, 1 \mathrm{H}), 7.64-7.60(\mathrm{~m}, 1 \mathrm{H}), 7.55(\mathrm{~s}, 1 \mathrm{H}), 2.69$ (s, 3H) . ${ }^{[5]}$

\section{Benzo $[h]$ furo $[2,3-f]$ isoquinoline (6a)}

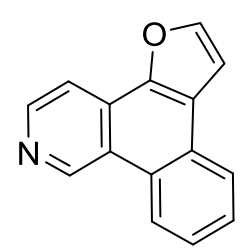

Yield: $74 \%$ (40mg). White solid. m.p. 98-99.1 ${ }^{\circ} \mathrm{C}$. Purified by flash column chromatography $($ EtOAc/hexane $=1 / 20) .{ }^{1} \mathrm{H}$ NMR $\left(400 \mathrm{MHz}, \mathrm{CDCl}_{3}\right) \delta 9.98(\mathrm{~s}, 1 \mathrm{H})$, $8.73(\mathrm{~d}, J=7.4 \mathrm{~Hz}, 2 \mathrm{H}), 8.12-8.00(\mathrm{~m}, 2 \mathrm{H}), 7.85(\mathrm{~d}, J=2.0 \mathrm{~Hz}, 1 \mathrm{H}), 7.65(\mathrm{ddd}, J=7.0$, 4.9, $1.6 \mathrm{~Hz}, 2 \mathrm{H}), 7.23(\mathrm{~d}, J=2.1 \mathrm{~Hz}, 1 \mathrm{H}) .{ }^{13} \mathrm{C} \mathrm{NMR}\left(101 \mathrm{MHz}, \mathrm{CDCl}_{3}\right) \delta 147.0,146.9$, 145.8, 145.7, 127.9, 127.6, 127.1, 126.4, 126.3, 124.3, 124.2, 123.0, 113.6, 106.7; IR (KBr), v ( $\left.\mathrm{cm}^{-1}\right)$ 3448, 1631, 1076, 611; HRMS (ESI) m/z calcd for. $\mathrm{C}_{15} \mathrm{H}_{9} \mathrm{NO}[\mathrm{M}+\mathrm{H}]^{+} 220.0757$, found 220.0751 .

\section{5-Methoxybenzo $[h]$ furo[2,3- $f]$ isoquinoline $(6 \mathrm{~b})$}

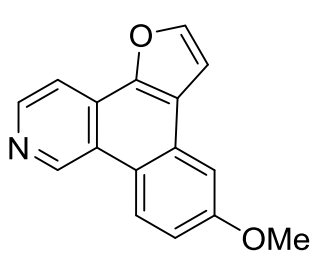

Yield: $79 \%$ (49mg). White solid. m.p. $132.5-133.5{ }^{\circ} \mathrm{C}$. Purified by flash column chromatography $($ EtOAc/hexane $=1 / 20) .{ }^{1} \mathrm{H}$ NMR $\left(400 \mathrm{MHz}, \mathrm{CDCl}_{3}\right) \delta 9.85(\mathrm{~s}$, $1 \mathrm{H}), 8.68(\mathrm{~d}, J=5.4 \mathrm{~Hz}, 1 \mathrm{H}), 8.59(\mathrm{~d}, J=9.1 \mathrm{~Hz}, 1 \mathrm{H}), 7.99(\mathrm{~d}, J=5.3 \mathrm{~Hz}, 1 \mathrm{H})$,

$7.83(\mathrm{~d}, J=2.0 \mathrm{~Hz}, 1 \mathrm{H}), 7.39$ (d, $J=2.6 \mathrm{~Hz}, 1 \mathrm{H}), 7.23(\mathrm{dd}, J=9.0,2.6 \mathrm{~Hz}, 1 \mathrm{H})$, $7.17(\mathrm{~d}, J=2.0 \mathrm{~Hz}, 1 \mathrm{H}), 3.98(\mathrm{~s}, 3 \mathrm{H}) .{ }^{13} \mathrm{C} \mathrm{NMR}\left(101 \mathrm{MHz}, \mathrm{CDCl}_{3}\right) \delta 159.3,147.3,146.5,145.6,144.6$, 129.1, 125.1, 124.5, 123.8, 123.5, 120.9, 115.9, 113.6, 106.6, 105.6, 55.6; IR (KBr), v ( $\left.\mathrm{cm}^{-1}\right) 3436$, 3087, 2590, 1915, 1614,1230, 1037, 819, 586; HRMS (ESI) m/z calcd for. $\mathrm{C}_{16} \mathrm{H}_{11} \mathrm{NO}_{2}[\mathrm{M}+\mathrm{H}]^{+}$ 250.0863 , found 250.0856 .

\section{5-Methylbenzo[ $h]$ furo[2,3-f]isoquinoline (6c)}

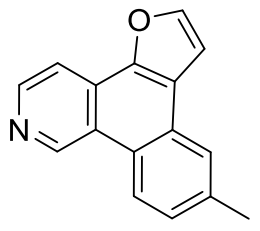

Yield: $70 \%$ (41mg). White solid. m.p. 98.6-100 ${ }^{\circ} \mathrm{C}$. Purified by flash column chromatography $($ EtOAc/hexane $=1 / 20) .{ }^{1} \mathrm{H}$ NMR $\left(400 \mathrm{MHz}, \mathrm{CDCl}_{3}\right) \delta 9.97(\mathrm{~s}, 1 \mathrm{H})$, $8.74(\mathrm{~d}, J=5.5 \mathrm{~Hz}, 1 \mathrm{H}), 8.65(\mathrm{~d}, J=8.4 \mathrm{~Hz}, 1 \mathrm{H}), 8.06(\mathrm{~d}, J=5.4 \mathrm{~Hz}, 1 \mathrm{H}), 7.97-7.81$ $(\mathrm{m}, 2 \mathrm{H}), 7.49(\mathrm{~d}, J=8.5 \mathrm{~Hz}, 1 \mathrm{H}), 7.26(\mathrm{~d}, J=2.0 \mathrm{~Hz}, 1 \mathrm{H}), 2.61(\mathrm{~s}, 3 \mathrm{H}) .{ }^{13} \mathrm{C}$ NMR $(101$ 
$\left.\mathrm{MHz}, \mathrm{CDCl}_{3}\right) \delta 147.1,146.8,145.8,145.2,138.0,128.1,127.8,124.9,124.1,124.1,123.5,122.9,113.7$, 106.7, 21.8; IR (KBr), $v\left(\mathrm{~cm}^{-1}\right) 3454,2923,1944,1618,1448,1249,1039,748,572 ; \mathrm{HRMS}$ (ESI) m/z calcd for. $\mathrm{C}_{16} \mathrm{H}_{11} \mathrm{NO}[\mathrm{M}+\mathrm{H}]^{+}$234.0913, found 234.0907.

\section{5-(Trifluoromethyl)benzo $[h]$ furo $[2,3-f]$ isoquinoline (6d)}

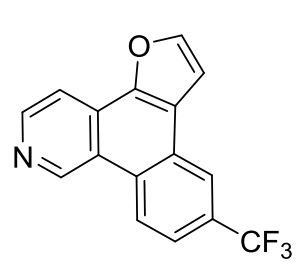

Yield: $41 \%$ (29mg). Yellow solid. m.p. $184-184.8{ }^{\circ} \mathrm{C}$. Purified by flash column chromatography $($ EtOAc/hexane $=1 / 20) .{ }^{1} \mathrm{H}$ NMR $(400 \mathrm{MHz}, \mathrm{DMSO}) \delta 10.16(\mathrm{~s}$, $1 \mathrm{H}), 9.14(\mathrm{~d}, J=8.7 \mathrm{~Hz}, 1 \mathrm{H}), 8.83(\mathrm{~d}, J=5.4 \mathrm{~Hz}, 1 \mathrm{H}), 8.70(\mathrm{~s}, 1 \mathrm{H}), 8.36(\mathrm{~s}, 1 \mathrm{H})$,

$8.12(\mathrm{~d}, J=5.3 \mathrm{~Hz}, 1 \mathrm{H}), 7.93(\mathrm{~d}, J=8.5 \mathrm{~Hz}, 1 \mathrm{H}), 7.85(\mathrm{~s}, 1 \mathrm{H}) .13 \mathrm{C}$ NMR $(101$ MHz, DMSO) $\delta 148.0,147.3,146.3,145.8,129.0,128.3\left(\mathrm{q},{ }^{2} J=32 \mathrm{~Hz}\right), 125.4\left(\mathrm{q},{ }^{1} J=270 \mathrm{~Hz}\right), 126.4$, 125.0, 124.5, 123.0, 122.2(q, $\left.{ }^{3} J=4 \mathrm{~Hz}\right), 122.0,121.9,113.6,107.6$. IR (KBr), $v\left(\mathrm{~cm}^{-1}\right) 2254,1650,997$, 765; HRMS (ESI) m/z calcd for. $\mathrm{C}_{16} \mathrm{H}_{8} \mathrm{~F}_{3} \mathrm{NO}[\mathrm{M}+\mathrm{H}]^{+} 288.0631$, found 288.0622.

\section{1-(Benzo[h]furo[2,3-f]isoquinolin-5-yl)ethan-1-one (6e)}

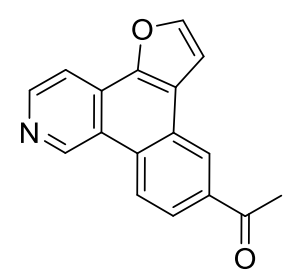

Yield: $45 \%$ (29mg). White solid. m.p. 117-168.2 ${ }^{\circ} \mathrm{C}$. Purified by flash column chromatography $($ EtOAc/hexane $=1 / 20) .{ }^{1} \mathrm{H}$ NMR $\left(600 \mathrm{MHz}, \mathrm{CDCl}_{3}\right) \delta 10.02(\mathrm{~s}$, $1 \mathrm{H}), 8.84(\mathrm{~d}, J=8.7 \mathrm{~Hz}, 2 \mathrm{H}), 8.71(\mathrm{~d}, J=2.0 \mathrm{~Hz}, 1 \mathrm{H}), 8.21(\mathrm{dd}, J=8.7,1.9 \mathrm{~Hz}, 1 \mathrm{H})$, $8.11(\mathrm{~d}, J=5.4 \mathrm{~Hz}, 1 \mathrm{H}), 7.95(\mathrm{~d}, J=2.1 \mathrm{~Hz}, 1 \mathrm{H}), 7.38(\mathrm{~d}, J=2.0 \mathrm{~Hz}, 1 \mathrm{H}), 2.80(\mathrm{~s}$, 3H). ${ }^{13} \mathrm{C} \mathrm{NMR}\left(151 \mathrm{MHz}, \mathrm{CDCl}_{3}\right) \delta 197.8,147.6,147.3,146.7,146.5,135.9,130.5,127.4,125.5,124.9$, 124.6, 123.5, 122.8, 113.9, 106.9, 27.1; IR (KBr), $v\left(\mathrm{~cm}^{-1}\right)$ 3448, 2921, 1672, 1253, 1105, 825, 597; HRMS (ESI) m/z calcd for. $\mathrm{C}_{17} \mathrm{H}_{11} \mathrm{NO}_{2}[\mathrm{M}+\mathrm{H}]^{+}$262.0863, found 262.0860.

\section{2,5-Dimethylbenzo[ $h]$ thieno[2,3- $f]$ isoquinoline (6f)}

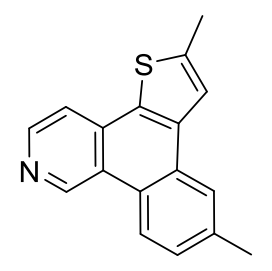

Yield: $68 \%$ (45mg). White solid. m.p. 106-107.8 ${ }^{\circ} \mathrm{C}$. Purified by flash column chromatography $($ EtOAc/hexane $=1 / 20) .{ }^{1} \mathrm{H}$ NMR $\left(400 \mathrm{MHz}, \mathrm{CDCl}_{3}\right) \delta 9.86(\mathrm{~s}, 1 \mathrm{H})$, $8.61(\mathrm{~d}, J=5.5 \mathrm{~Hz}, 1 \mathrm{H}), 8.56(\mathrm{~d}, J=8.4 \mathrm{~Hz}, 1 \mathrm{H}), 7.95(\mathrm{~s}, 1 \mathrm{H}), 7.72(\mathrm{~d}, J=5.4 \mathrm{~Hz}$,

$1 \mathrm{H}), 7.55(\mathrm{~d}, J=1.4 \mathrm{~Hz}, 1 \mathrm{H}), 7.45(\mathrm{dd}, J=8.4,1.8 \mathrm{~Hz}, 1 \mathrm{H}), 2.70(\mathrm{~s}, 3 \mathrm{H}), 2.58(\mathrm{~s}, 3 \mathrm{H})$.

${ }^{13} \mathrm{C}$ NMR $\left(101 \mathrm{MHz}, \mathrm{CDCl}_{3}\right) \delta 146.6,144.7,143.2,138.8,137.9,132.4,129.0,128.5,125.4,124.4$, 122.7, 121.6, 117.1, 21.9, 16.5; IR (KBr), $v\left(\mathrm{~cm}^{-1}\right)$ 3446, 2852, 2254, 1649, 997, 613; HRMS (ESI) $\mathrm{m} / \mathrm{z}$ calcd for. $\mathrm{C}_{17} \mathrm{H}_{13} \mathrm{NS}[\mathrm{M}+\mathrm{H}]^{+} 264.0841$,found 264.0847 .

\section{1-(5-Methylbenzo[ $h]$ thieno[2,3-f]isoquinolin-2-yl)ethan-1-one $(6 \mathrm{~g})$}

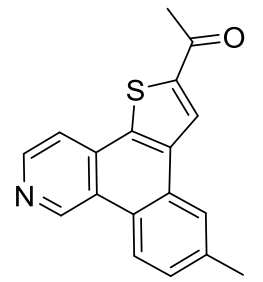

Yield: 64\% (46mg). White solid. m.p. 212-214 ${ }^{\circ} \mathrm{C}$. Purified by flash column chromatography $($ EtOAc/hexane $=1 / 20) .{ }^{1} \mathrm{H}$ NMR $\left(400 \mathrm{MHz}, \mathrm{CDCl}_{3}\right) \delta 9.92(\mathrm{~s}, 1 \mathrm{H})$, $8.81-8.67(\mathrm{~m}, 1 \mathrm{H}), 8.59(\mathrm{~d}, J=8.4 \mathrm{~Hz}, 1 \mathrm{H}), 8.42(\mathrm{~s}, 1 \mathrm{H}), 8.04(\mathrm{~s}, 1 \mathrm{H}), 7.85$ (d, $J=5.4$ $\mathrm{Hz}, 1 \mathrm{H}), 7.52(\mathrm{~d}, J=8.3 \mathrm{~Hz}, 1 \mathrm{H}), 2.75(\mathrm{~s}, 3 \mathrm{H}), 2.62(\mathrm{~s}, 3 \mathrm{H}) \cdot{ }^{13} \mathrm{C} \mathrm{NMR}(101 \mathrm{MHz}$, $\left.\mathrm{CDCl}_{3}\right) \delta 191.4,147.2,145.7,144.8,138.7,137.9,131.8,129.3,129.2,127.8,125.6,124.4,124.1$, 123.0, 117.6, 27.1, 21.9. IR (KBr), $v\left(\mathrm{~cm}^{-1}\right) 3440,2923,1643,995,767$; HRMS (ESI) m/z calcd for. $\mathrm{C}_{18} \mathrm{H}_{13} \mathrm{NOS}[\mathrm{M}+\mathrm{H}]^{+}$292.0791, found 292.0795. 


\section{7-Methylbenzo[h]furo[2,3-f]isoquinoline $(6 \mathrm{~h})$}

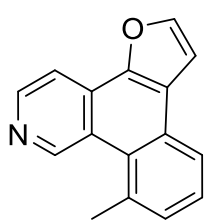

Yield: $85 \%$ (50mg). White solid. m.p. 153.9-154.5 ${ }^{\circ} \mathrm{C}$. Purified by flash column chromatography $($ EtOAc/hexane $=1 / 20) .{ }^{1} \mathrm{H}$ NMR $\left(400 \mathrm{MHz}, \mathrm{CDCl}_{3}\right) \delta 10.16(\mathrm{~s}, 1 \mathrm{H})$, $8.74(\mathrm{~d}, J=5.3 \mathrm{~Hz}, 1 \mathrm{H}), 8.10(\mathrm{~d}, J=5.3 \mathrm{~Hz}, 1 \mathrm{H}), 8.01(\mathrm{dd}, J=7.7,1.5 \mathrm{~Hz}, 1 \mathrm{H}), 7.83(\mathrm{~d}, J$ $=2.0 \mathrm{~Hz}, 1 \mathrm{H}), 7.55(\mathrm{~d}, J=7.6 \mathrm{~Hz}, 1 \mathrm{H}), 7.53-7.46(\mathrm{~m}, 1 \mathrm{H}), 7.22(\mathrm{~d}, J=2.0 \mathrm{~Hz}, 1 \mathrm{H}), 3.13$ (s, 3H). ${ }^{13} \mathrm{C}$ NMR $\left(101 \mathrm{MHz}, \mathrm{CDCl}_{3}\right) \delta$ 150.4, 146.7, 145.8, 144.7, 136.2, 131.1, 128.8, 127.4, 127.1, 124.7, 122.7, 113.5, 106.9, 27.9; IR (KBr), $v\left(\mathrm{~cm}^{-1}\right)$ 3749, 3446, 3093, 1726, 1446, 1342, 983, 775, 601; HRMS (ESI) m/z calcd for. $\mathrm{C}_{16} \mathrm{H}_{11} \mathrm{NO}[\mathrm{M}+\mathrm{H}]^{+} 234.0913$, found 234.0912.

\section{Benzo $[h]$ benzofuro[ $[2,3-f]$ isoquinoline (6i)}

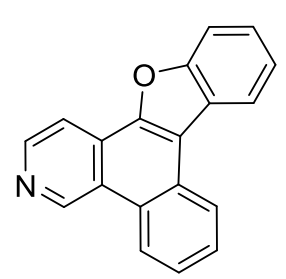

Yield: 51\% (34mg). White solid. m.p. $163.2-163.8{ }^{\circ} \mathrm{C}$. Purified by flash column chromatography $($ EtOAc/hexane $=1 / 20) .{ }^{1} \mathrm{H}$ NMR $\left(400 \mathrm{MHz}, \mathrm{CDCl}_{3}\right) \delta 9.99(\mathrm{~s}, 1 \mathrm{H})$, $8.82-8.73(\mathrm{~m}, 2 \mathrm{H}), 8.57-8.51(\mathrm{~m}, 1 \mathrm{H}), 8.30(\mathrm{~d}, J=7.6 \mathrm{~Hz}, 1 \mathrm{H}), 8.16(\mathrm{~d}, J=5.4 \mathrm{~Hz}$, $1 \mathrm{H}), 7.81-7.66(\mathrm{~m}, 3 \mathrm{H}), 7.58-7.45(\mathrm{~m}, 2 \mathrm{H}) .{ }^{13} \mathrm{C} \mathrm{NMR}\left(101 \mathrm{MHz}, \mathrm{CDCl}_{3}\right) \delta 156.3$, 148.6, 146.6, 145.2, 128.6, 128.4, 127.0, 127.0, 126.3, 126.2, 124.7, 124.5, 124.4, 123.9, 123.1, 122.4, 118.5, 114.6, 112.4, 77.5, 77.2, 76.8; IR (KBr), $v\left(\mathrm{~cm}^{-1}\right) 3452,1635,997,613$; HRMS (ESI) m/z calcd for. $\mathrm{C}_{19} \mathrm{H}_{11} \mathrm{NO}[\mathrm{M}+\mathrm{H}]^{+} 270.0913$, found 270.0913 .

\section{cis-8b,13a-Dihydrobenzo $[h]$ benzofuro $[2,3-f]$ isoquinoline $(6 \mathbf{j})$}

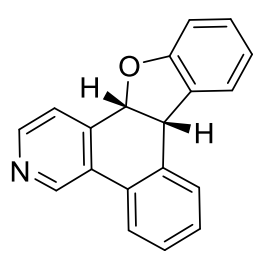

Yield: $20 \%$ (13mg). White solid. m.p. $139-140.5^{\circ} \mathrm{C}$. Purified by flash column chromatography $($ EtOAc/hexane $=1 / 20) .{ }^{1} \mathrm{H} \mathrm{NMR}\left(600 \mathrm{MHz}, \mathrm{CDCl}_{3}\right) \delta 9.07(\mathrm{~s}, 1 \mathrm{H})$, $8.61-8.50(\mathrm{~m}, 1 \mathrm{H}), 7.96(\mathrm{~d}, J=6.8 \mathrm{~Hz}, 1 \mathrm{H}), 7.62(\mathrm{~d}, J=4.7 \mathrm{~Hz}, 1 \mathrm{H}), 7.50(\mathrm{~d}, J=$ $7.8 \mathrm{~Hz}, 1 \mathrm{H}), 7.43(\mathrm{~d}, J=5.1 \mathrm{~Hz}, 2 \mathrm{H}), 7.14-7.04$ (m, 2H), $6.86(\mathrm{~d}, J=7.3 \mathrm{~Hz}, 2 \mathrm{H})$, $6.01(\mathrm{~d}, J=9.2 \mathrm{~Hz}, 1 \mathrm{H}), 4.85(\mathrm{~d}, J=9.1 \mathrm{~Hz}, 1 \mathrm{H}) .{ }^{13} \mathrm{C} \mathrm{NMR}\left(151 \mathrm{MHz}, \mathrm{CDCl}_{3}\right) \delta 158.2,148.9,144.7$, 141.3, 132.1, 1304, 129.3, 129.2 , 128.9, 128.8, 128.4, 128.1, 124.4, 123.5, 123.0, 121.6, 110.3, 79.8, 44.7; IR (KBr), $v\left(\mathrm{~cm}^{-1}\right) 3456,1631,1082,605$; HRMS (ESI) $\mathrm{m} / \mathrm{z}$ calcd for. $\mathrm{C}_{19} \mathrm{H}_{13} \mathrm{NO}[\mathrm{M}+\mathrm{H}]^{+}$ 272.1070, found 272.1071 .

\section{Benzo[h] furo[2,3-f]quinazoline (8a)}

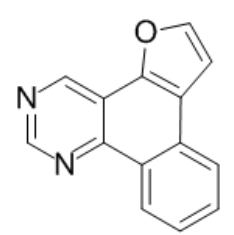

Yield: 79\% (43 mg). White solid. m.p. 163.9-164.5 ${ }^{\circ} \mathrm{C}$. Purified by flash column chromatography $($ EtOAc/hexane $=1 / 20) .{ }^{1} \mathrm{H} \mathrm{NMR}\left(400 \mathrm{MHz}, \mathrm{CDCl}_{3}\right) \delta 9.76(\mathrm{~s}, 1 \mathrm{H})$, 9.48 (s, 1H), $9.30(\mathrm{~d}, J=8.2 \mathrm{~Hz}, 1 \mathrm{H}), 8.15(\mathrm{~d}, J=8.0 \mathrm{~Hz}, 1 \mathrm{H}), 7.91(\mathrm{~d}, J=2.0 \mathrm{~Hz}, 1 \mathrm{H})$,

$7.88-7.82(\mathrm{~m}, 1 \mathrm{H}), 7.77-7.71(\mathrm{~m}, 1 \mathrm{H}), 7.32(\mathrm{~d}, J=2.0 \mathrm{~Hz}, 1 \mathrm{H}) .{ }^{13} \mathrm{C}$ NMR $(101 \mathrm{MHz}$, $\left.\mathrm{CDCl}_{3}\right) \delta 154.9,151.5,149.4,145.9,145.5,130.9,130.4,127.7,126.3,126.2,123.7,122.3,114.8$, 106.6. IR (KBr), $v\left(\mathrm{~cm}^{-1}\right) 3419,2923,1564,1342,1143,744$; HRMS (ESI) m/z calcd for. $\mathrm{C}_{14} \mathrm{H}_{8} \mathrm{~N}_{2} \mathrm{O}$ $[\mathrm{M}+\mathrm{H}]^{+}$221.0709, found 221.0709.

\section{Benzo $[h]$ thieno $[2,3-f]$ quinazoline $(8 b)$}




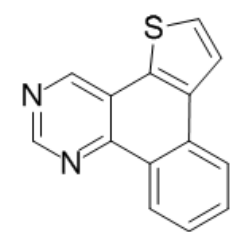

Yield: $65 \%$ (38 mg). Yellow solid. m.p. 183.0-184.1 ${ }^{\circ} \mathrm{C}$. Purified by flash column chromatography $($ EtOAc/hexane $=1 / 20) .{ }^{1} \mathrm{H}$ NMR $\left(400 \mathrm{MHz}, \mathrm{CDCl}_{3}\right) \delta 9.56(\mathrm{~s}, 1 \mathrm{H})$, $9.46(\mathrm{~s}, 1 \mathrm{H}), 9.28(\mathrm{~d}, J=8.2 \mathrm{~Hz}, 1 \mathrm{H}), 8.30(\mathrm{~d}, J=8.1 \mathrm{~Hz}, 1 \mathrm{H}), 7.99(\mathrm{~d}, J=5.3 \mathrm{~Hz}, 1 \mathrm{H})$, $7.85(\mathrm{dt}, J=8.3,4.3 \mathrm{~Hz}, 1 \mathrm{H}), 7.78-7.73(\mathrm{~m}, 1 \mathrm{H}), 7.71(\mathrm{~d}, J=5.3 \mathrm{~Hz}, 1 \mathrm{H}) .{ }^{13} \mathrm{C}$ NMR $\left(101 \mathrm{MHz}, \mathrm{CDCl}_{3}\right) \delta 155.4,154.1,149.7,136.8,131.9,131.1,128.4,127.3,127.0,126.0,124.0,123.1$, 120.6. IR (KBr), $v\left(\mathrm{~cm}^{-1}\right) 3749,2923,1739,1419,1340,1118,732,621$; HRMS (ESI) m/z calcd for. $\mathrm{C}_{14} \mathrm{H}_{8} \mathrm{~N}_{2} \mathrm{~S}[\mathrm{M}+\mathrm{H}]^{+} 237.0481$, found 237.0478.

\section{6-Methylbenzo[ $h]$ furo[2,3-f]quinazoline $(8 \mathrm{c})$}

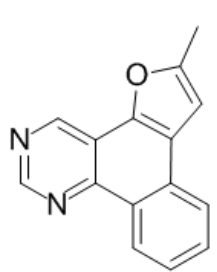

Yield: $68 \%(39 \mathrm{mg})$. Yellow solid. m.p. 117.0-118.7 ${ }^{\circ} \mathrm{C}$. Purified by flash column chromatography $($ EtOAc/hexane $=1 / 20) .{ }^{1} \mathrm{H}$ NMR $\left(400 \mathrm{MHz}, \mathrm{CDCl}_{3}\right) \delta 9.61(\mathrm{~s}, 1 \mathrm{H})$, $9.40(\mathrm{~s}, 1 \mathrm{H}), 9.22(\mathrm{~d}, J=8.1 \mathrm{~Hz}, 1 \mathrm{H}), 7.98(\mathrm{~d}, J=7.8 \mathrm{~Hz}, 1 \mathrm{H}), 7.78(\mathrm{t}, J=7.3 \mathrm{~Hz}, 1 \mathrm{H})$, $7.67(\mathrm{t}, J=7.5 \mathrm{~Hz}, 1 \mathrm{H}), 6.81(\mathrm{~s}, 1 \mathrm{H}), 2.59(\mathrm{~s}, 3 \mathrm{H}) .{ }^{13} \mathrm{C} \mathrm{NMR}\left(101 \mathrm{MHz}, \mathrm{CDCl}_{3}\right) \delta$ 156.6, 154.1, 151.0, 148.5, 144.3, 130.6, 130.2, 127.4, 125.9, 125.9, 123.6, 114.4, 102.7, 14.4. IR (KBr), $v\left(\mathrm{~cm}^{-1}\right) 3450,2918,1560,1429,1134,761,617$; HRMS (ESI) $\mathrm{m} / \mathrm{z}$ calcd for. $\mathrm{C}_{15} \mathrm{H}_{10} \mathrm{~N}_{2} \mathrm{O}[\mathrm{M}+\mathrm{H}]^{+}$ 235.0866, found 235.0864.

\section{6-Methylbenzo[ $h]$ thieno[2,3-f]quinazoline (8d)}

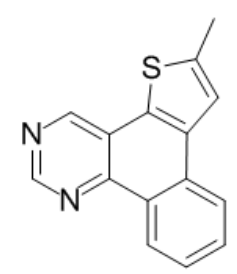

Yield: $61 \%$ (38 mg). Yellow solid. m.p. 161.9-163.6 ${ }^{\circ} \mathrm{C}$. Purified by flash column chromatography $($ EtOAc/hexane $=1 / 20) .{ }^{1} \mathrm{H}$ NMR $\left(400 \mathrm{MHz}, \mathrm{CDCl}_{3}\right) \delta 9.41(\mathrm{~d}, J=4.7$ $\mathrm{Hz}, 2 \mathrm{H}), 9.24(\mathrm{~d}, J=8.1 \mathrm{~Hz}, 1 \mathrm{H}), 8.17(\mathrm{~d}, J=8.0 \mathrm{~Hz}, 1 \mathrm{H}), 7.81(\mathrm{t}, J=7.5 \mathrm{~Hz}, 1 \mathrm{H}), 7.71$ $(\mathrm{t}, J=7.6 \mathrm{~Hz}, 1 \mathrm{H}), 7.59(\mathrm{~s}, 1 \mathrm{H}), 2.72(\mathrm{~s}, 3 \mathrm{H}) .{ }^{13} \mathrm{C} \mathrm{NMR}\left(101 \mathrm{MHz}, \mathrm{CDCl}_{3}\right) \delta 154.8$, 153.7, 149.2, 142.5, 137.1, 131.6, 130.9, 130.6, 128.4, 126.7, 125.9, 123.9, 121.1, 120.5, 16.4. IR (KBr), $v\left(\mathrm{~cm}^{-1}\right) 3452,2923,1562,1419,1118,619$; HRMS (ESI) m/z calcd for. $\mathrm{C}_{15} \mathrm{H}_{10} \mathrm{~N}_{2} \mathrm{~S}[\mathrm{M}+\mathrm{H}]^{+} 251.0637$, found 251.0635 .

\section{S10. References}

[1] A. N. Shestakov, A. S. Pankova, M. A. Kuznetsov, Chem., Heterocycl., Compd., 2017, 53, 1103-1113.

[2] F. Alonso, I. P. Beletskaya, M. Yus, Tetrahedron 2008, 64, 3047-3101.

[3] I. B. Seiple, S. Su, R. A. Rodriguez, R. Gianatassio, Y. Fujiwara, A. L. Sobel, P. S. Baran, J. Am. Chem. Soc., 2010, 132, 13194-13196.

[4] S. Chiba, Y.-J. Xu, Y.-F. Wang, J. Am. Chem. Soc., 2009, 131, 12886-12887.

[5] W. Hagui, N. Besbes, E. Srasra, T. Roisnel, J. F. Soule, H. Doucet, Org. Lett., 2016, 18, $4182-4185$. 
S11. X-ray Parameters and Structures of $2 \mathrm{~s}, 2 \mathrm{t}$ and $2 v$

trans-Dihydrobenzo[ $f]$ benzofuro $[3,2-h]$ quinoline (2s)

Identification code

Empirical formula

CCDC No.

Formula weight

Temperature/K

Crystal system

Space group

$\mathrm{a} / \AA$

$\mathrm{b} / \AA$

$c / \AA$

$\alpha /{ }^{\circ}$

$\beta /{ }^{\circ}$

$\gamma /{ }^{\circ}$

Volume $/ \AA^{3}$

Z

$\rho_{\text {calc } g / \mathrm{cm}^{3}}$

$\mu / \mathrm{mm}^{-1}$

$\mathrm{F}(000)$

Crystal size $/ \mathrm{mm}^{3}$

Radiation

$2 \Theta$ range for data collection ${ }^{\circ}$

Index ranges

Reflections collected

Independent reflections

Data/restraints/parameters

Goodness-of-fit on $\mathrm{F}^{2}$

Final $\mathrm{R}$ indexes [all data]

Largest diff. peak/hole / e $\AA^{-3}$
$2 s$

$\mathrm{C}_{19} \mathrm{H}_{13} \mathrm{NO}$

1911401

271.30

153

monoclinic

$\mathrm{P} 2{ }_{1} / \mathrm{c}$ (No. 14)

14.251 (19)

11.267(3)

8.303(17)

90

102.645(17)

90

1300.8(5)

4

1.385

0.675

568.0

$0.5 \times 0.4 \times 0.3$

$\mathrm{CuK} \alpha(\lambda=1.54184)$

13.832 to 137.248

$0 \leq \mathrm{h} \leq 33,-13 \leq \mathrm{k} \leq 0,-10 \leq 1 \leq 9$

2345

2345 [ $\left.\mathrm{R}_{\text {sigma }}=0.0280\right]$

$2345 / 0 / 190$

1.172

$\mathrm{R}_{1}=0.0748, \mathrm{wR}_{2}=0.2197$

$0.42 /-0.34$

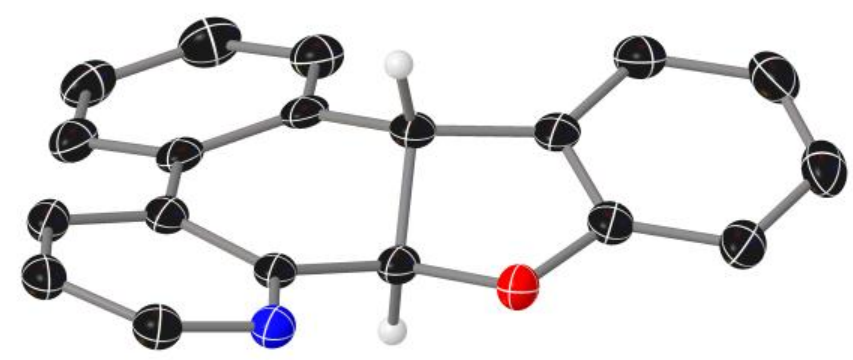

Figure S4 Thermal ellipsoid ORTEP diagram of 2s. 
cis-Dihydrobenzo[ $f]$ benzofuro[3,2- $h]$ quinoline (2t)

Identification code

Empirical formula

CCDC No.

Formula weight

Temperature/K

Crystal system

Space group

$\mathrm{a} / \AA$

$\mathrm{b} / \AA$

$\mathrm{c} / \AA$

$\alpha /{ }^{\circ}$

$\beta /{ }^{\circ}$

$\gamma /{ }^{\circ}$

Volume $/ \AA^{3}$

$\mathrm{Z}$

$\rho_{\text {calc }} \mathrm{g} / \mathrm{cm}^{3}$

$\mu / \mathrm{mm}^{-1}$

$\mathrm{F}(000)$

Crystal size $/ \mathrm{mm}^{3}$

Radiation

$2 \Theta$ range for data collection ${ }^{\circ}$

Index ranges

Reflections collected

Independent reflections

Reflections collected

Goodness-of-fit on $\mathrm{F}^{2}$

Final $\mathrm{R}$ indexes [all data]

Largest diff. peak/hole / e $\AA^{-3}$
$2 \mathrm{t}$

$\mathrm{C}_{19} \mathrm{H}_{13} \mathrm{NO}$

1934847

271.30

180

orthorhombic

P 212121 (No. 19)

5.10740(10)

$13.6665(2)$

$18.8236(3)$

90

90

90

1313.89(4)

4

1.372

0.668

568.0

$0.5 \times 0.4 \times 0.3$

$\mathrm{CuK} \alpha(\lambda=1.54178)$

3.939 to 75.832

$-6 \leq \mathrm{h} \leq 2,-16 \leq \mathrm{k} \leq 17,-23 \leq 1 \leq$

23

2572

$2572\left[\mathrm{R}_{\text {sigma }}=0.0410\right]$

$2572 / 0 / 190$

1.011

$\mathrm{R}_{1}=0.0356, \mathrm{wR}_{2}=0.1094$

$0.16 /-0.153$

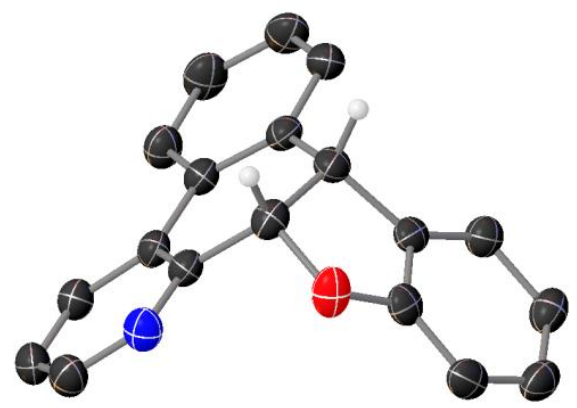

Figure S5 Thermal ellipsoid ORTEP diagram of 2t. 
cis-Methyl-dihydrobenzo[f]benzofuro[3,2- $h]$ quinoline (2v)

Identification code

Empirical formula

CCDC No.

Formula weight

Temperature/K

Crystal system

Space group

$\mathrm{a} / \AA$

$\mathrm{b} / \AA$

$\mathrm{c} / \AA$

$\alpha /{ }^{\circ}$

$\beta /{ }^{\circ}$

$\gamma /{ }^{\circ}$

Volume $/ \AA^{3}$

$\mathrm{Z}$

$\rho_{\text {calc }} \mathrm{g} / \mathrm{cm}^{3}$

$\mu / \mathrm{mm}^{-1}$

$\mathrm{F}(000)$

Crystal size $/ \mathrm{mm}^{3}$

Radiation

$2 \Theta$ range for data collection/ ${ }^{\circ}$

Index ranges

Reflections collected

Independent reflections

Reflections collected

Goodness-of-fit on $\mathrm{F}^{2}$

Final $\mathrm{R}$ indexes [all data]

Largest diff. peak/hole / e $\AA^{-3}$ 2v

$\mathrm{C}_{40} \mathrm{H}_{30} \mathrm{~N}_{2} \mathrm{O}_{2} \cdot \mathrm{H}_{2} \mathrm{O}$

1939728

294.34

180

orthorhombic

$\mathrm{P}$ ben (No. 60)

21.256

10.255

13.659

90

90

90

2977.449(14)

8

1.313

0.655

1240.0

$0.5 \times 0.4 \times 0.3$

$\mathrm{CuK} \alpha(\lambda=1.54178)$

3.1880 to 75.9230

$-26 \leq \mathrm{h} \leq 26,-12 \leq \mathrm{k} \leq 12,-17 \leq 1 \leq 17$

3103

$2696\left[\mathrm{R}_{\text {sigma }}=0.0094\right]$

$3103 / 3 / 214$

1.078

$\mathrm{R}_{1}=0.0361, \mathrm{wR}_{2}=0.0881$

$0.267 /-0.205$

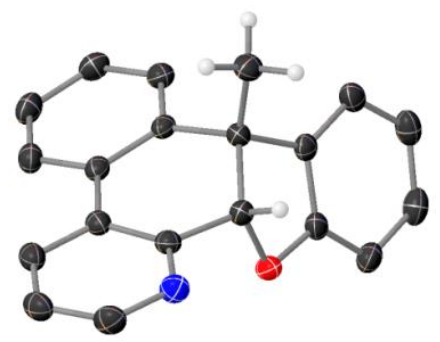

Figure S6 Thermal ellipsoid ORTEP diagram of $\mathbf{2 v}$. 
S12. ${ }^{1} \mathrm{H}$ NMR and ${ }^{13} \mathrm{C}$ NMR Spectra

Spectra of 12a-12n, 13a-13c, 14a-14d.

(12a)

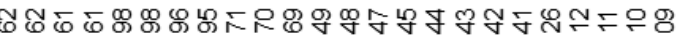

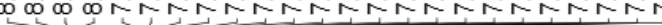

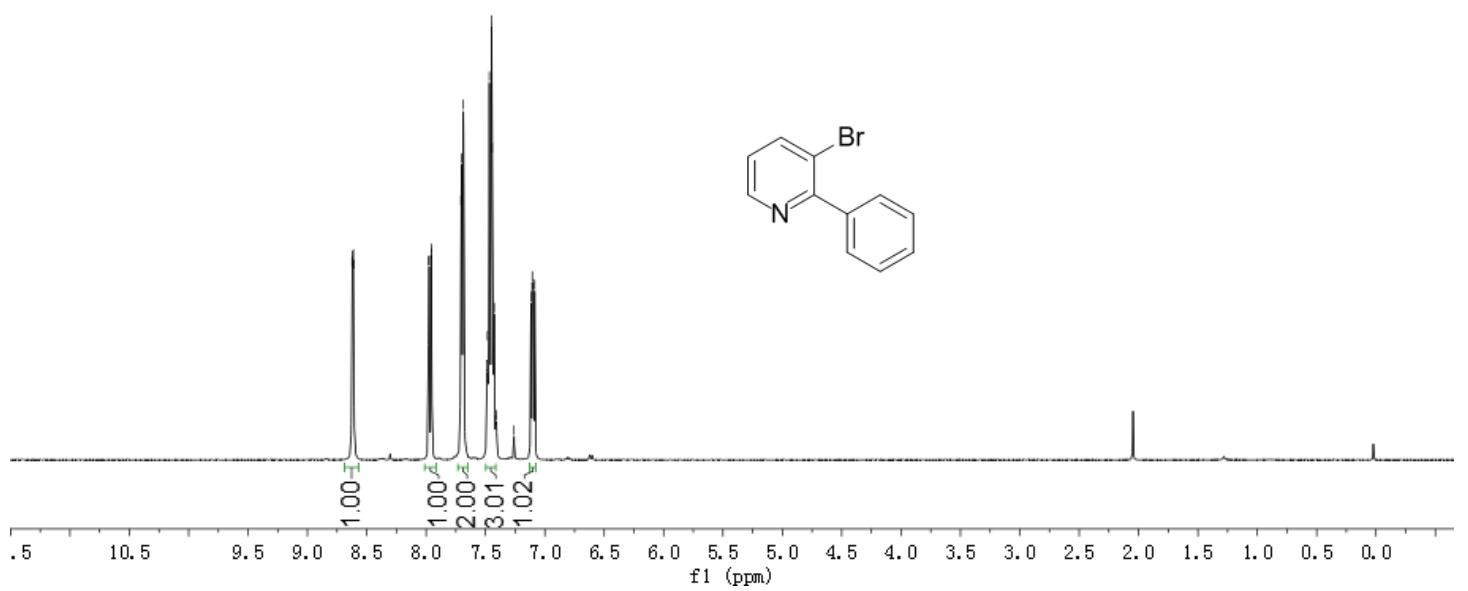

(12b)

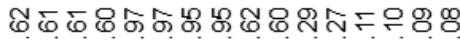
on on
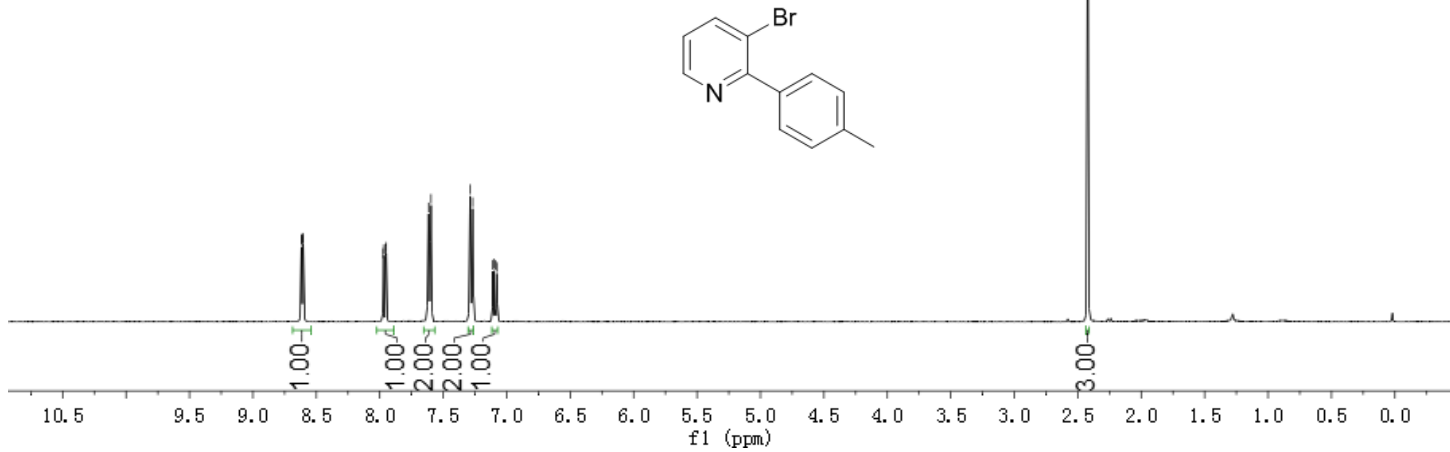
(12c)

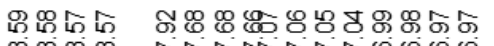

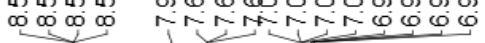

मे
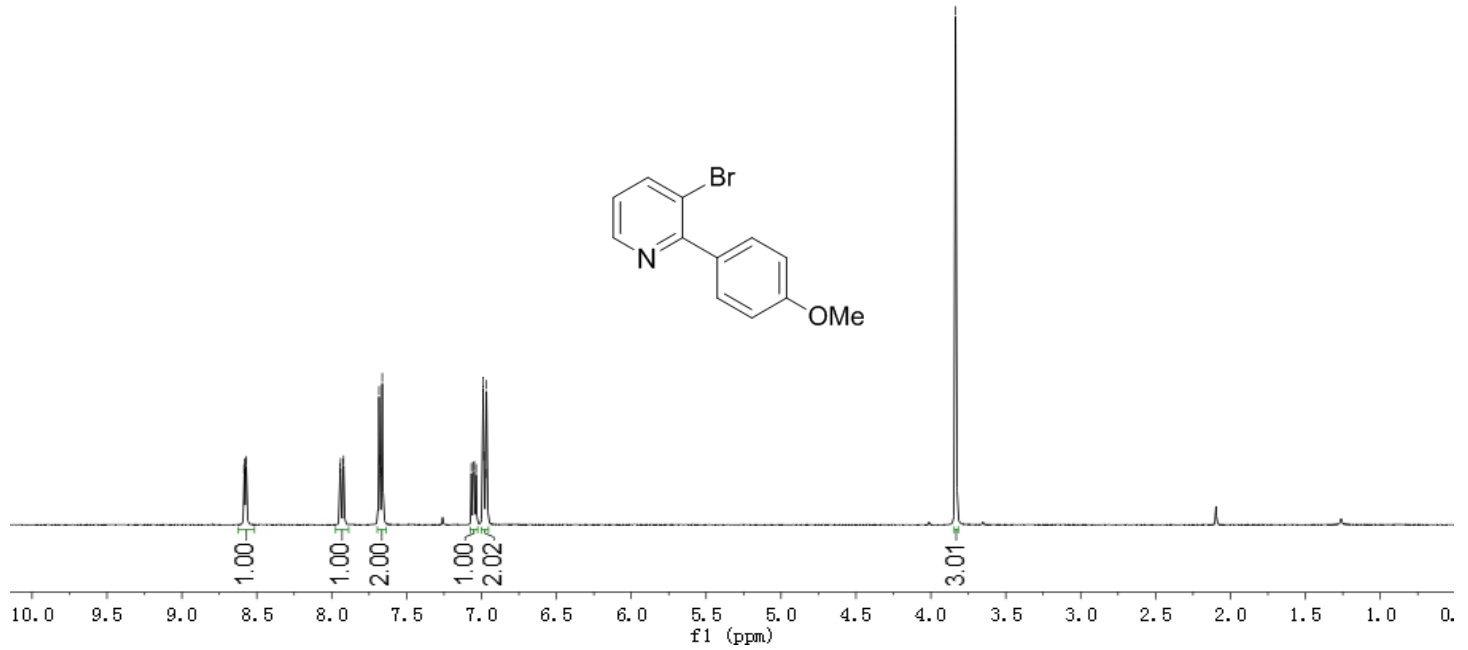

(12d)

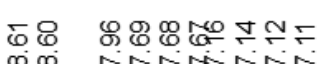

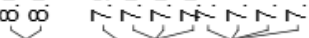

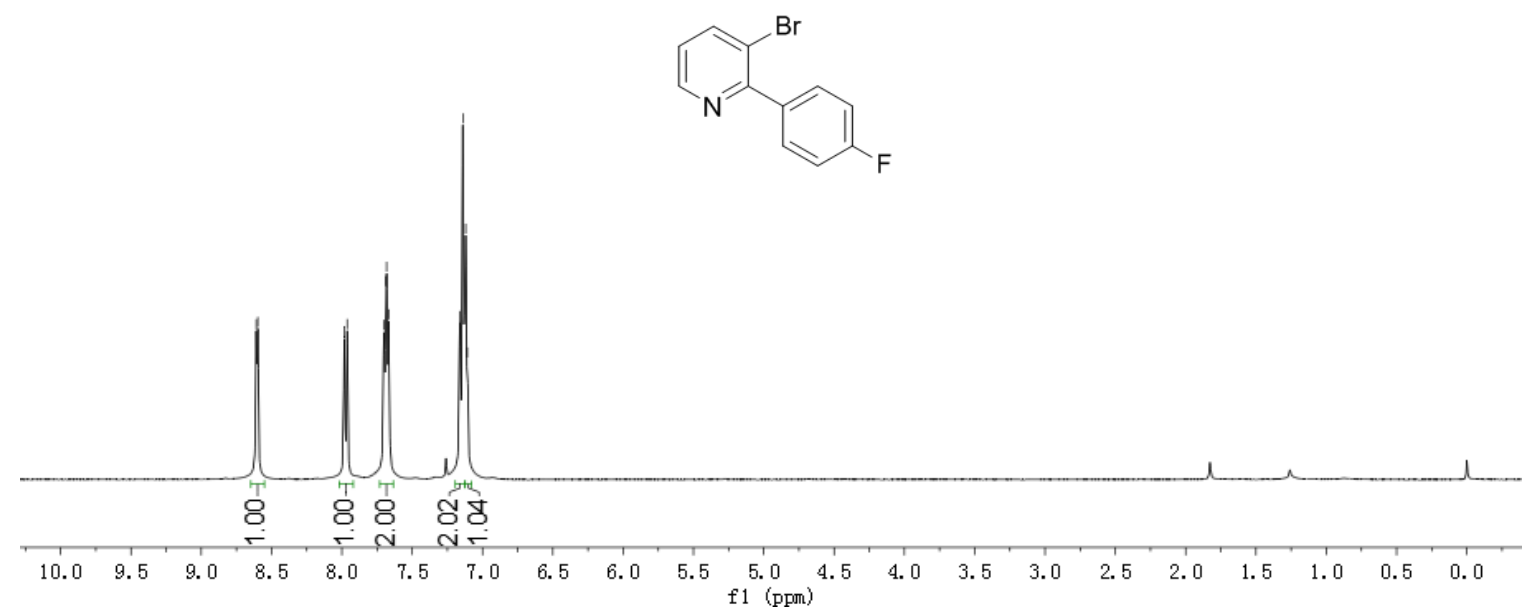


(12e)

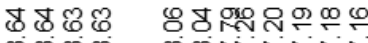

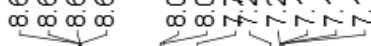

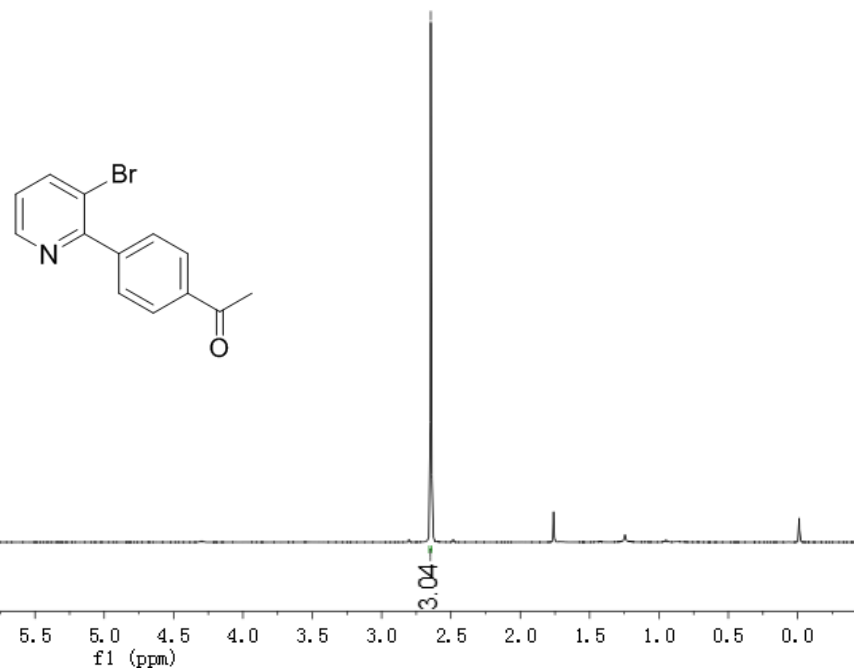

ஸั

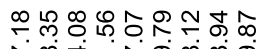

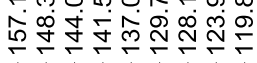

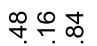

송

o.

广 广र्टर广रा

$\stackrel{\infty}{\sim}$
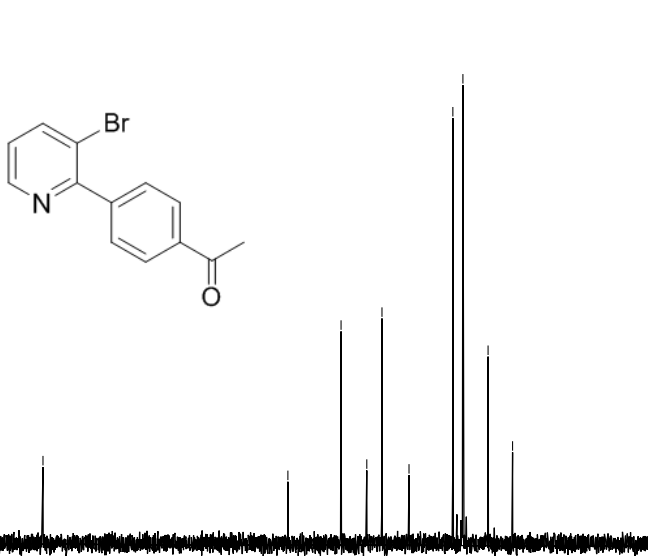

$\begin{array}{lllllllllllll}210 & 200 & 190 & 180 & 170 & 160 & 150 & 140 & 130 & 120 & 110 & 100 & 90\end{array}$ 


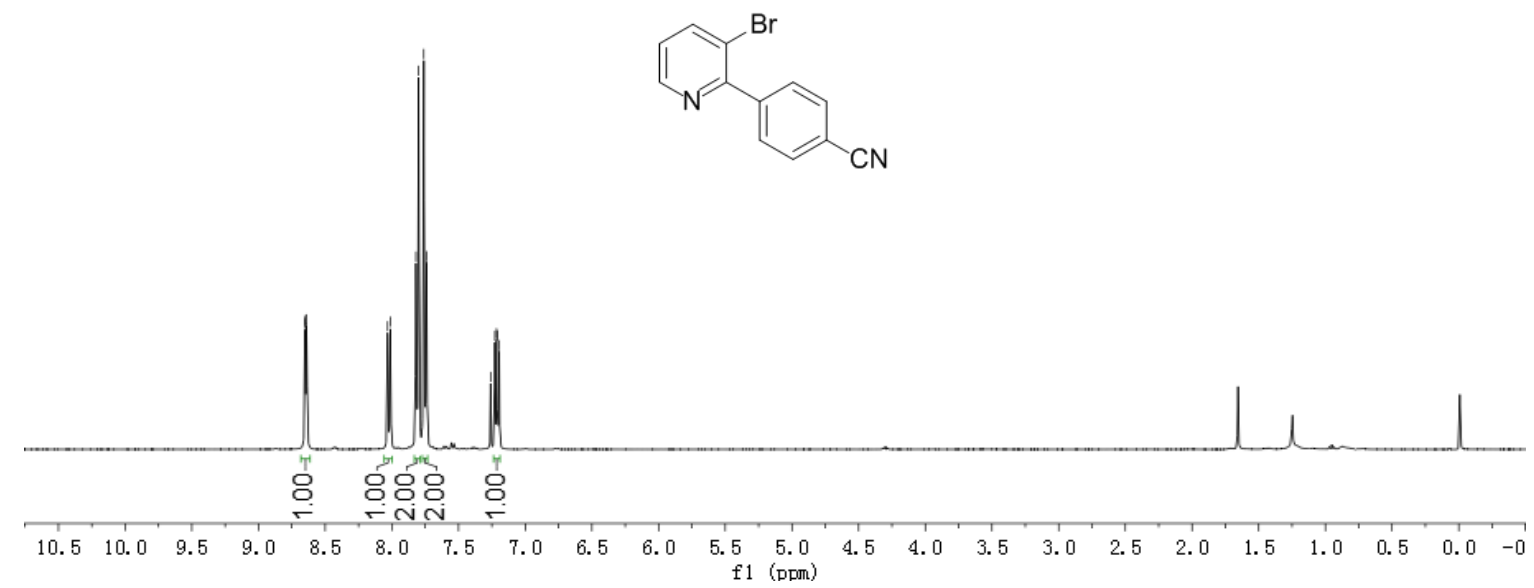

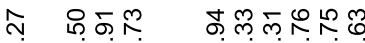

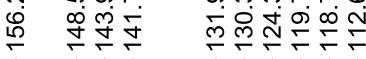

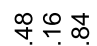

손

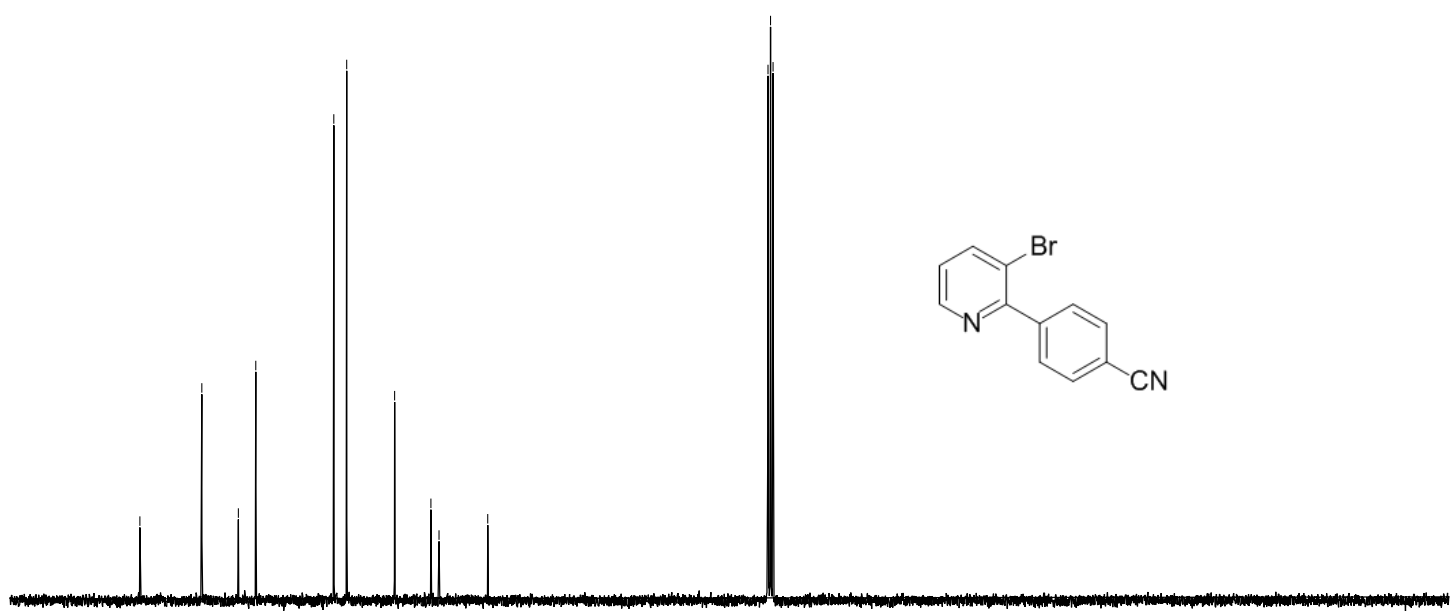


४षष৫

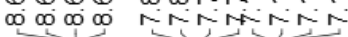

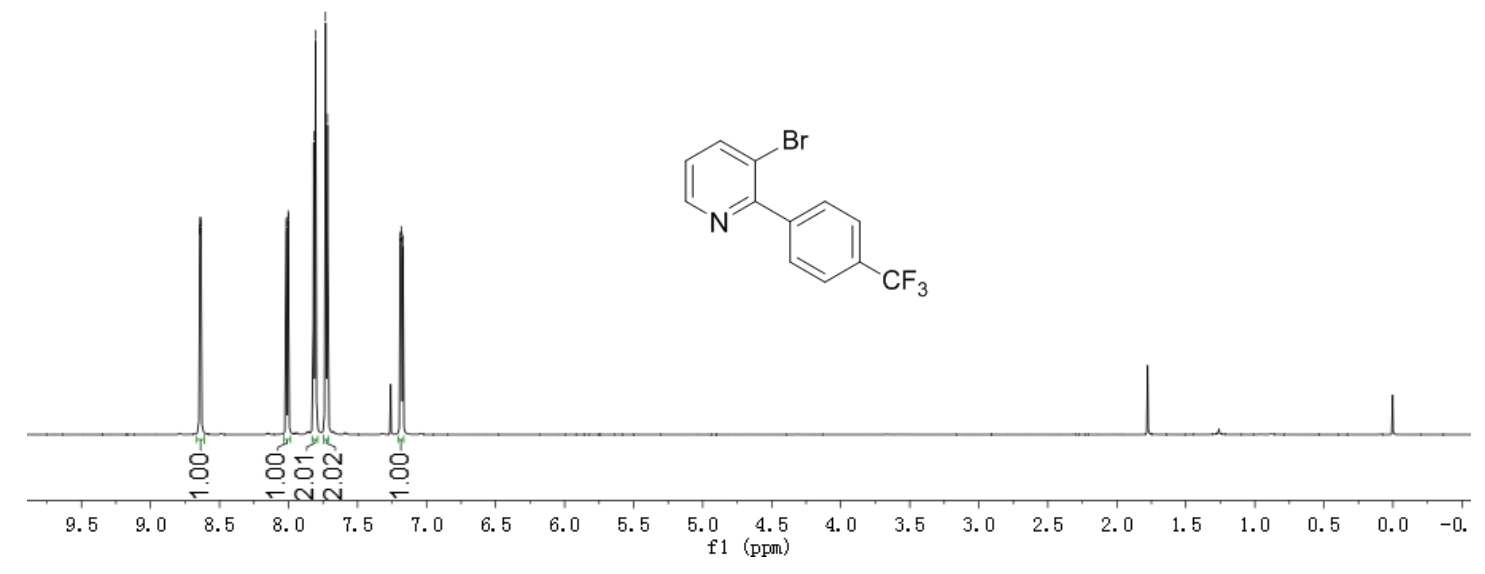

(12h)

४ ४

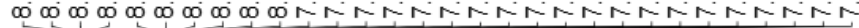

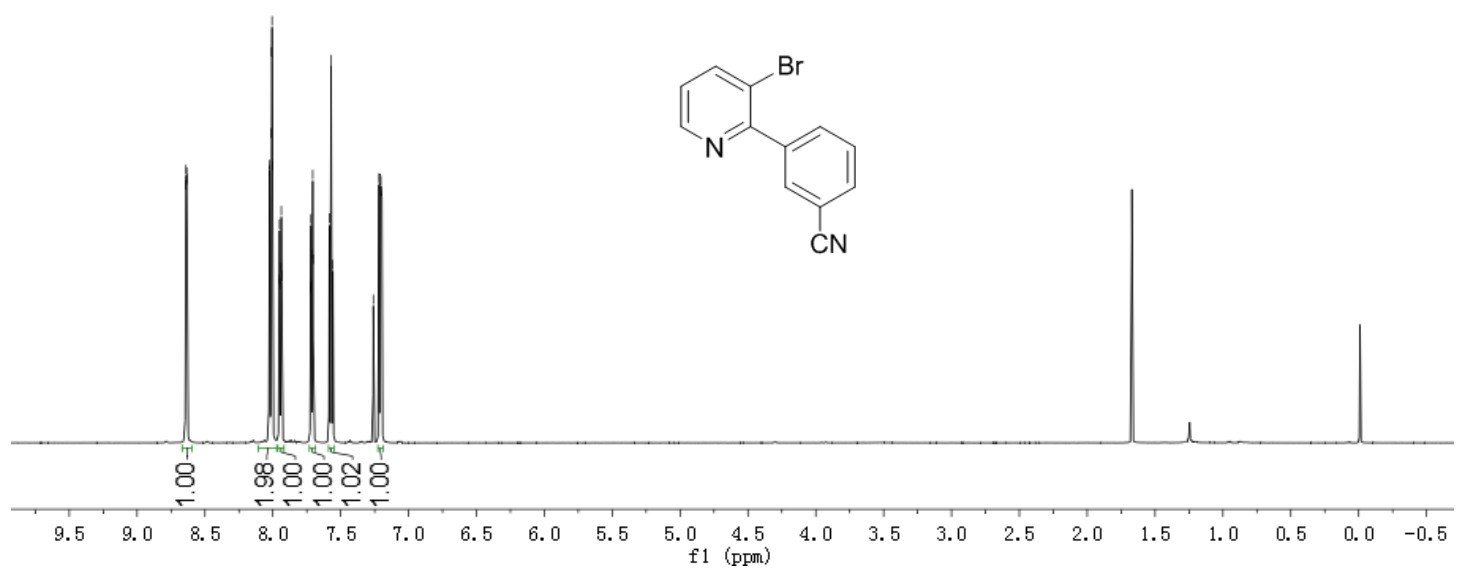




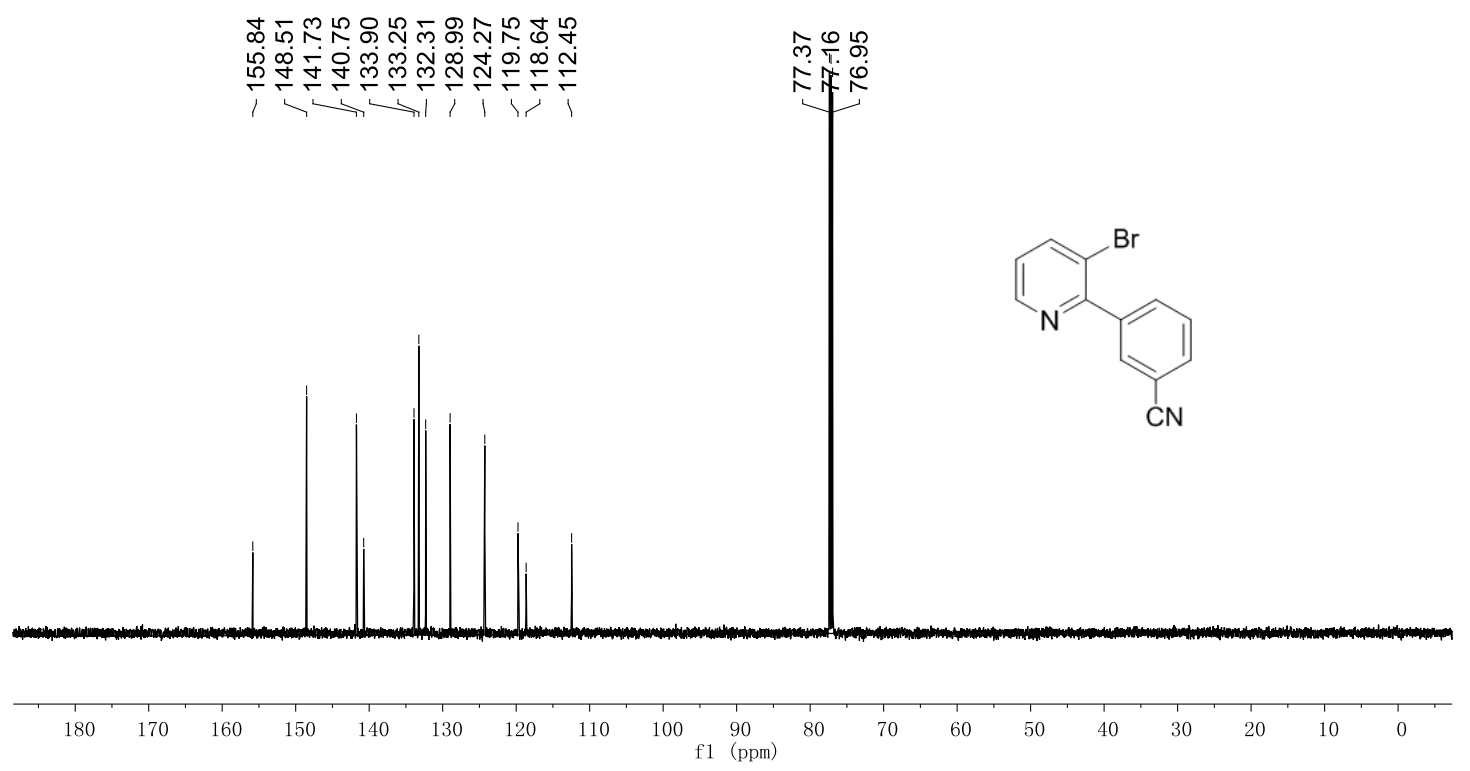

(12i)

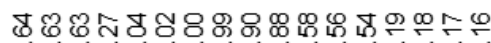
$\infty \omega^{\infty} \infty \omega_{0} \infty N-N N N N$
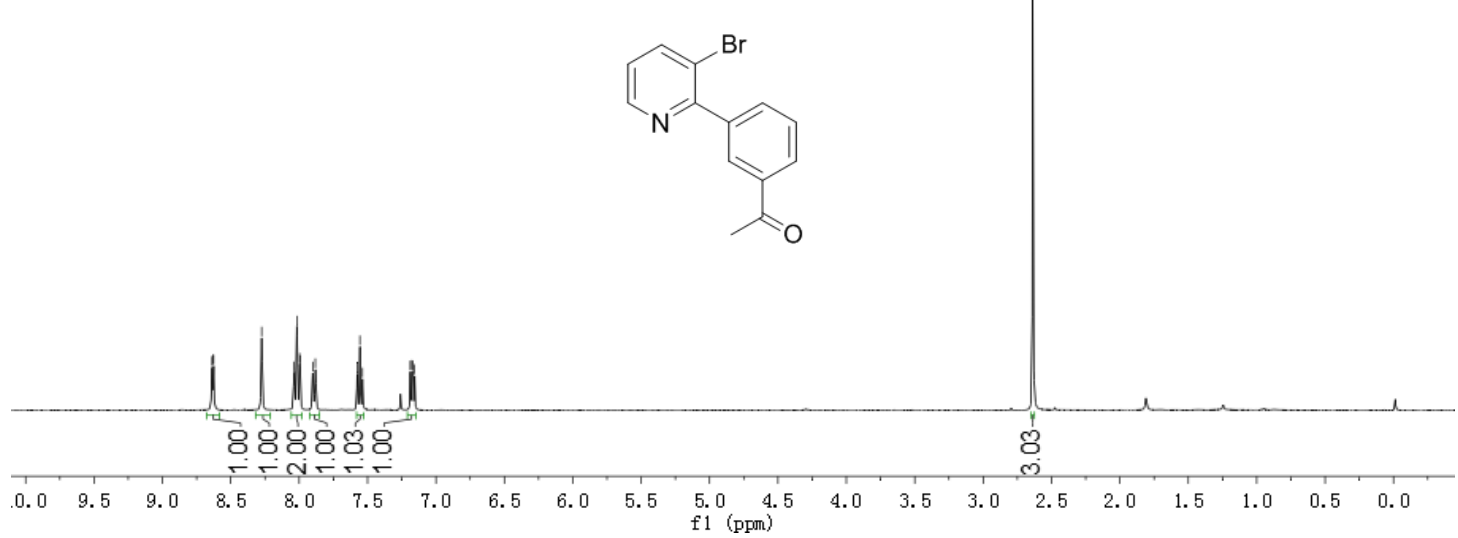


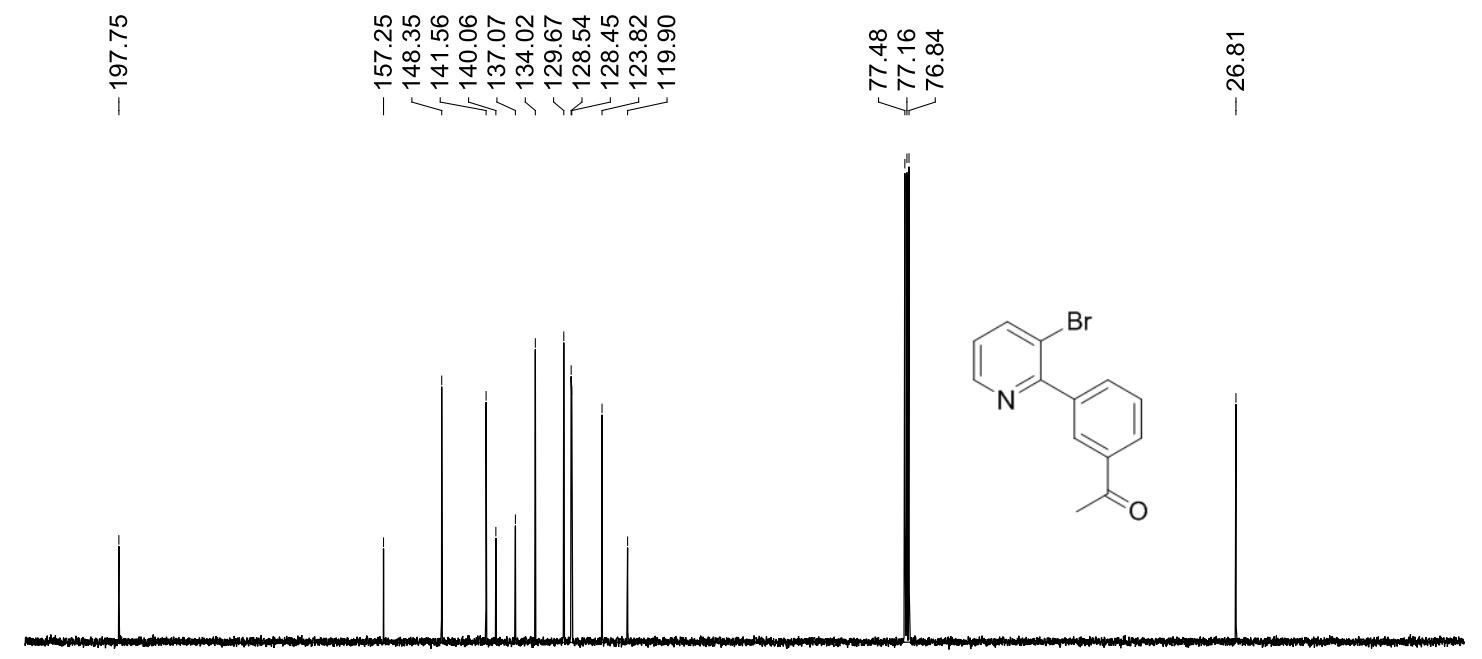

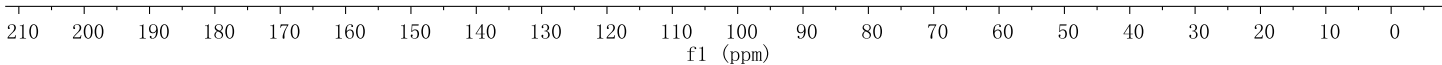

(12j)

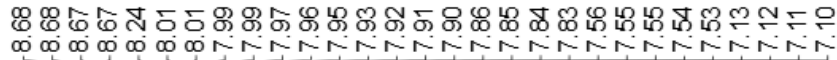

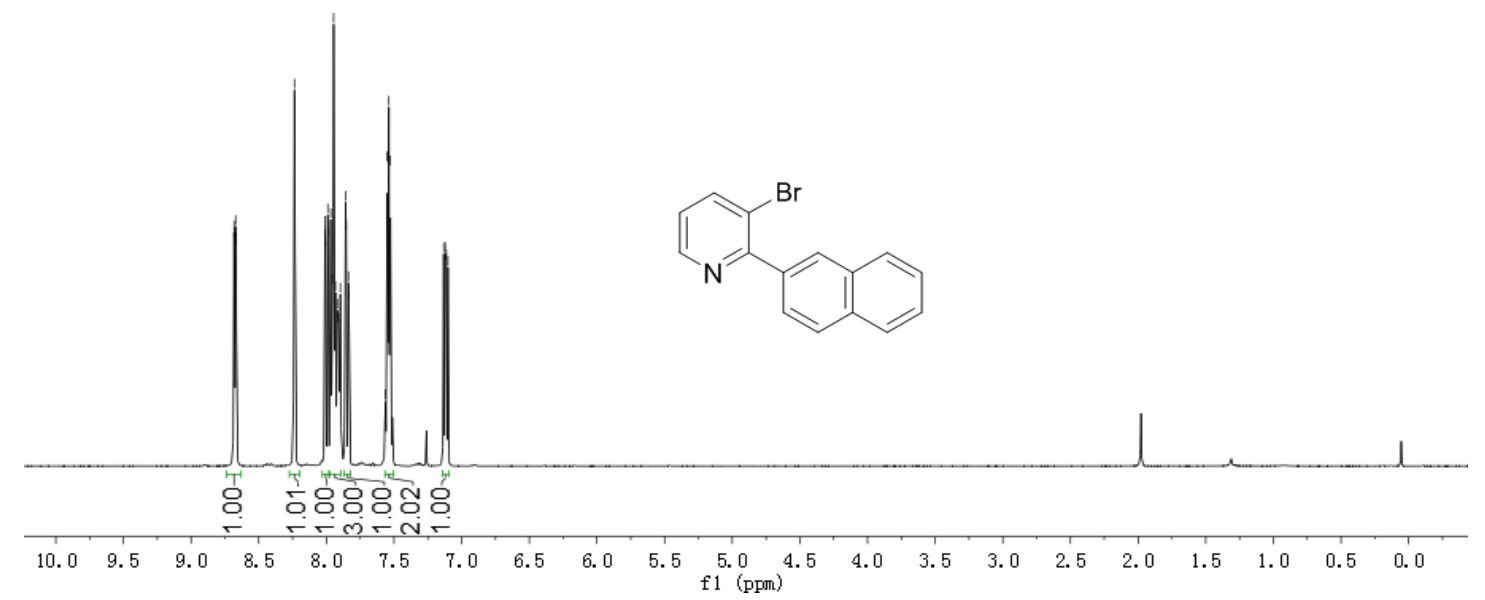




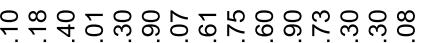

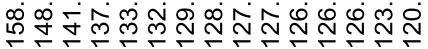

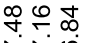

Кำ

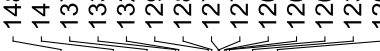
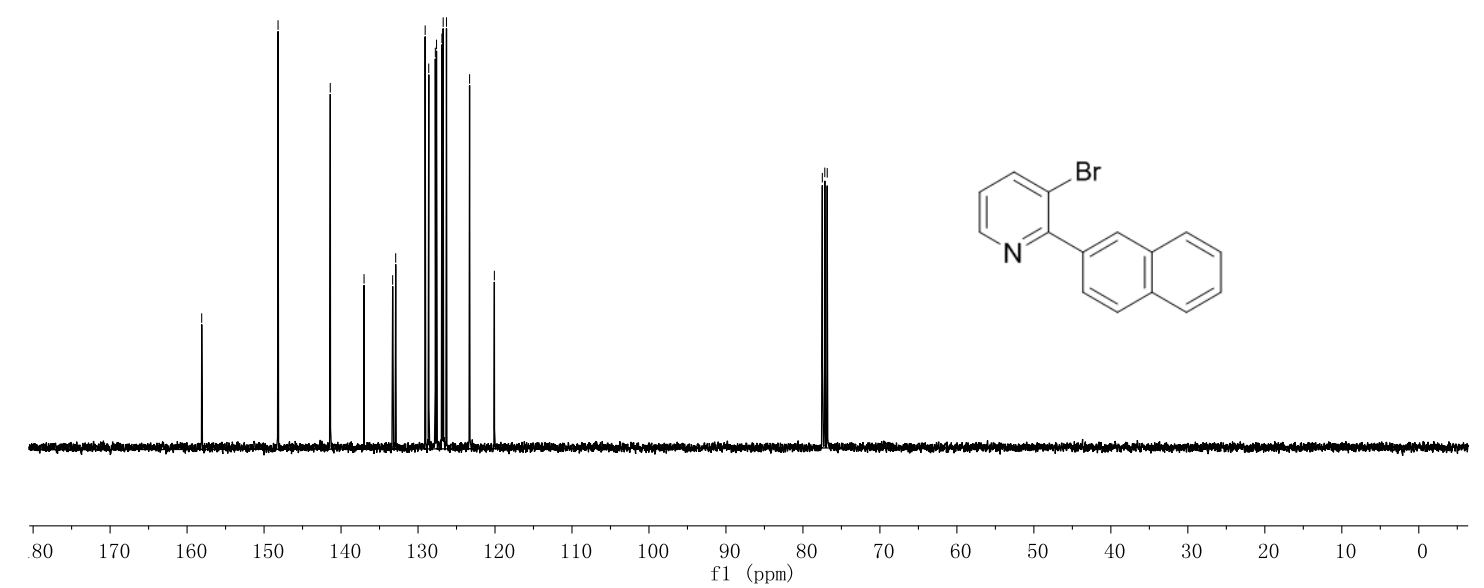

(12k)

ใ क⿻上丨

o vinitis

$\stackrel{\text { m }}{\text { i }}$

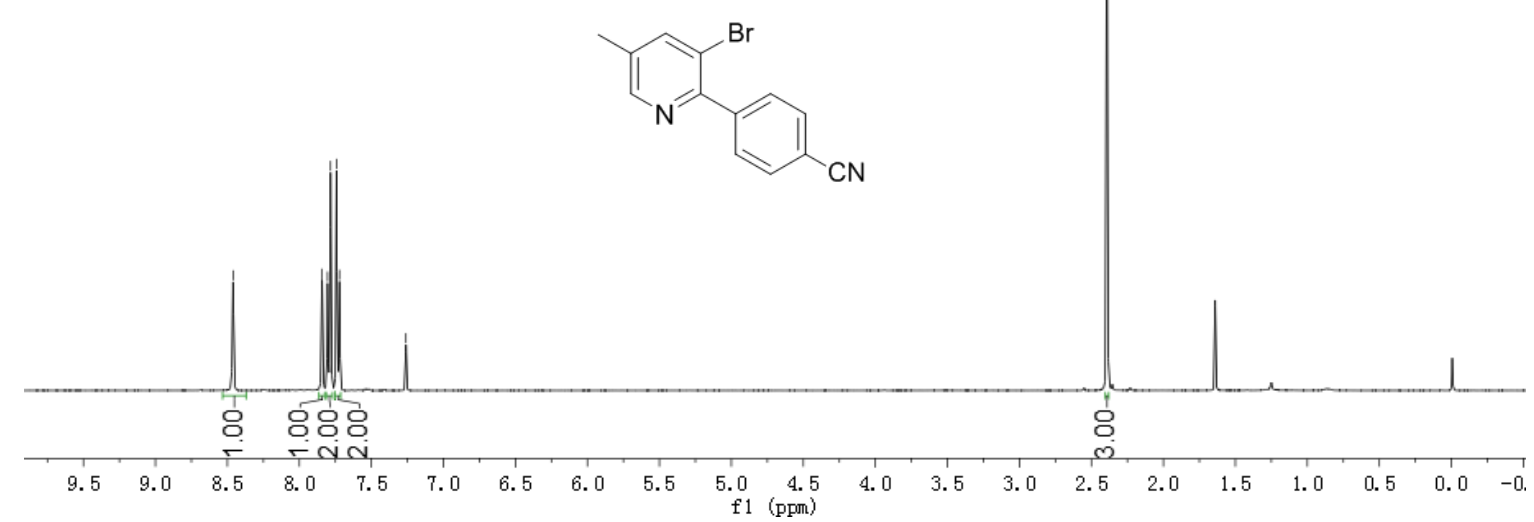




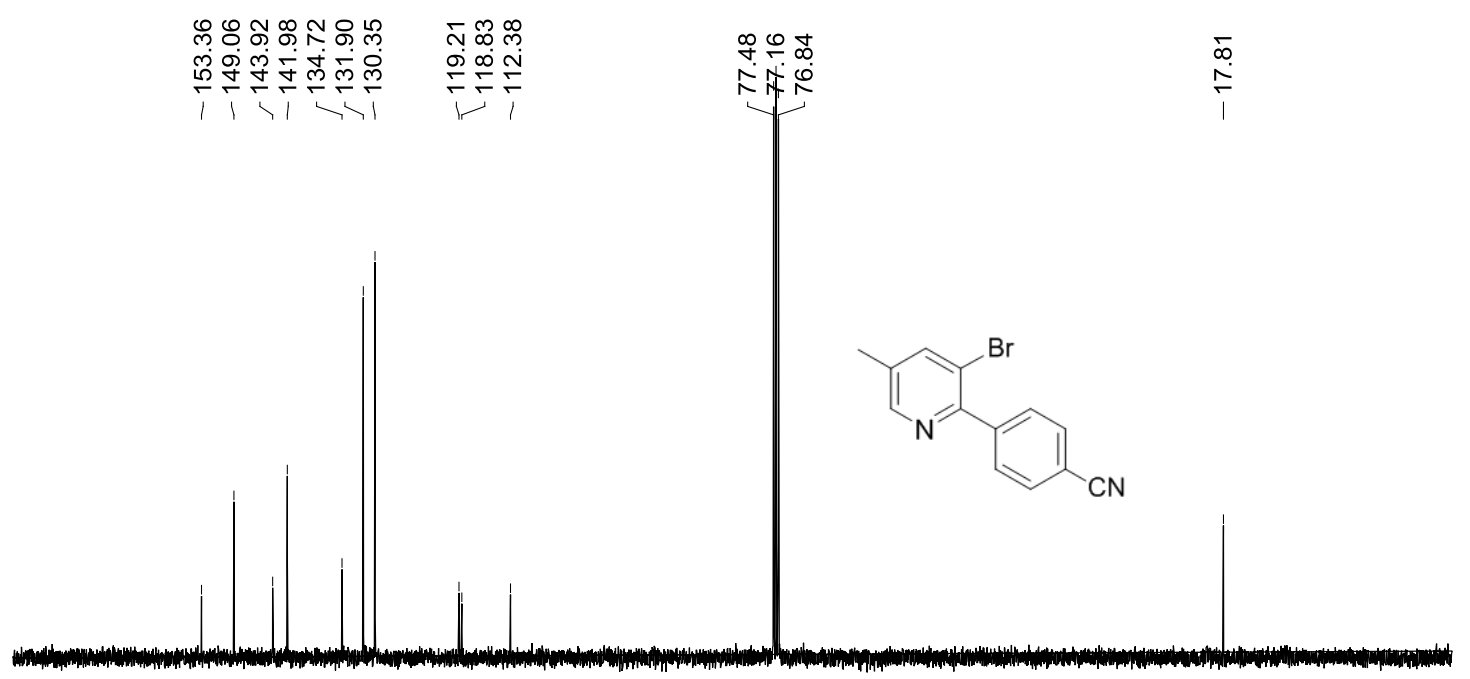

$\begin{array}{llllllllll}170 & 160 & 150 & 140 & 130 & 120 & 110 & 100 & 90 & 80 \\ & & & & & & & & \end{array}$

(12I)

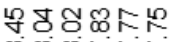

$\infty \underbrace{\infty}$

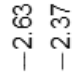

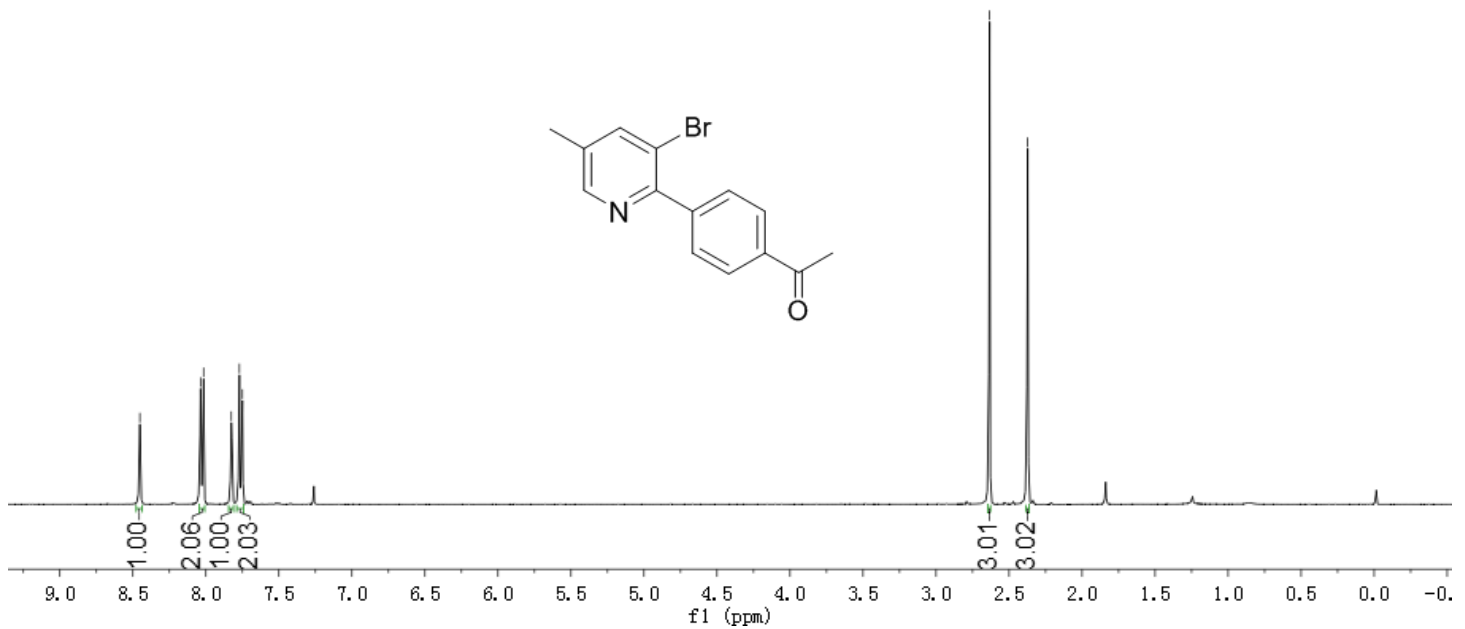




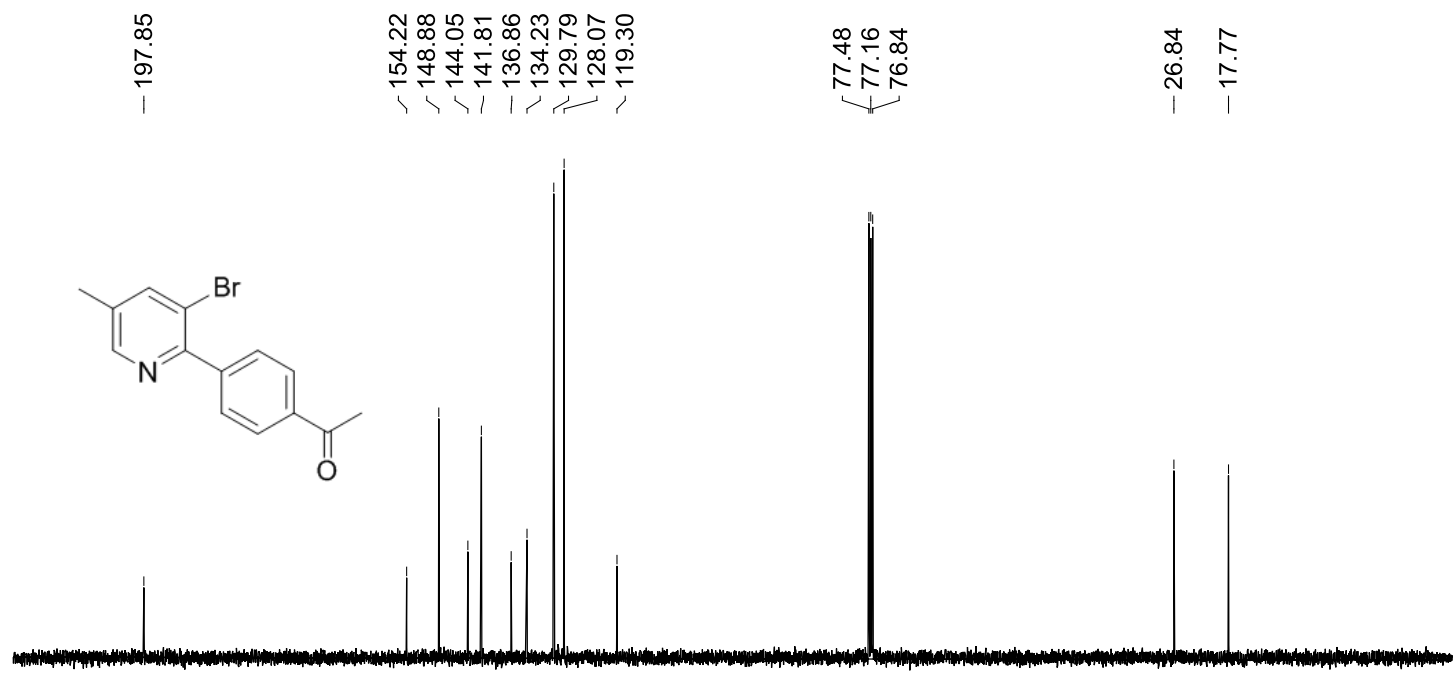

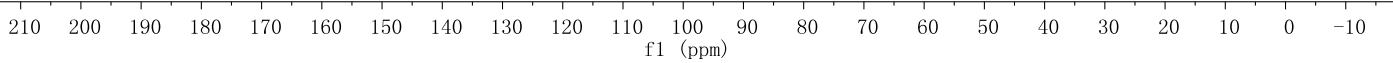

(12m)

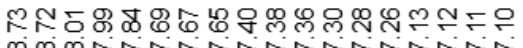

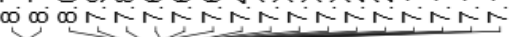

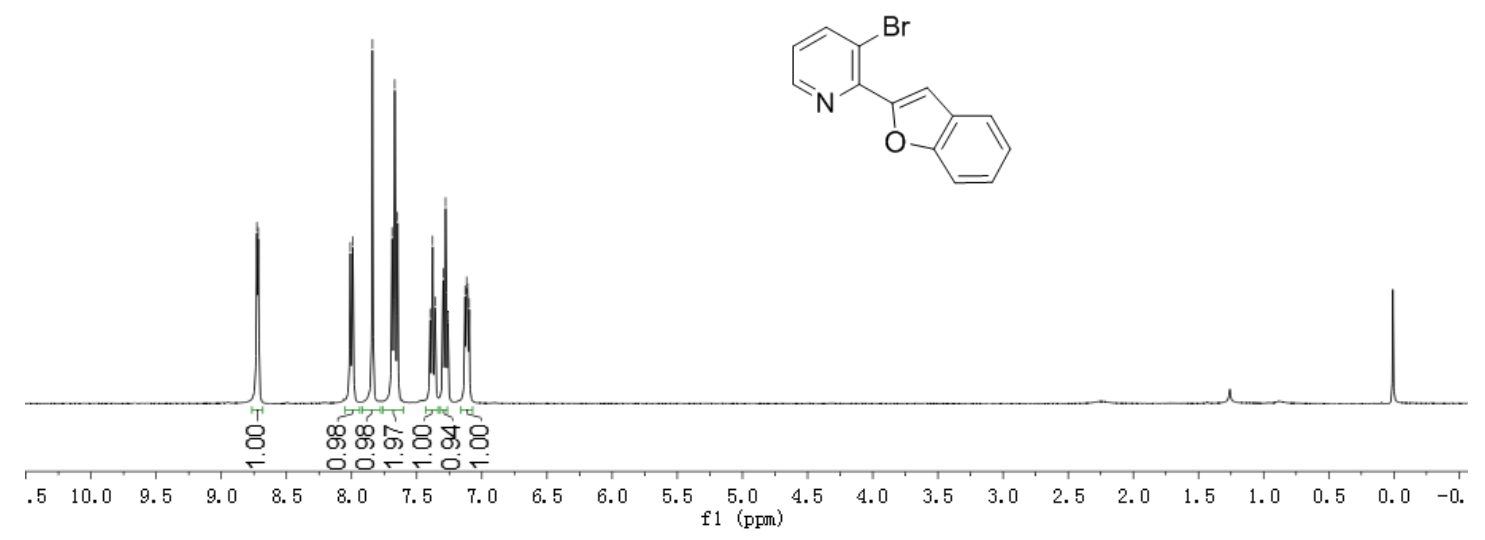




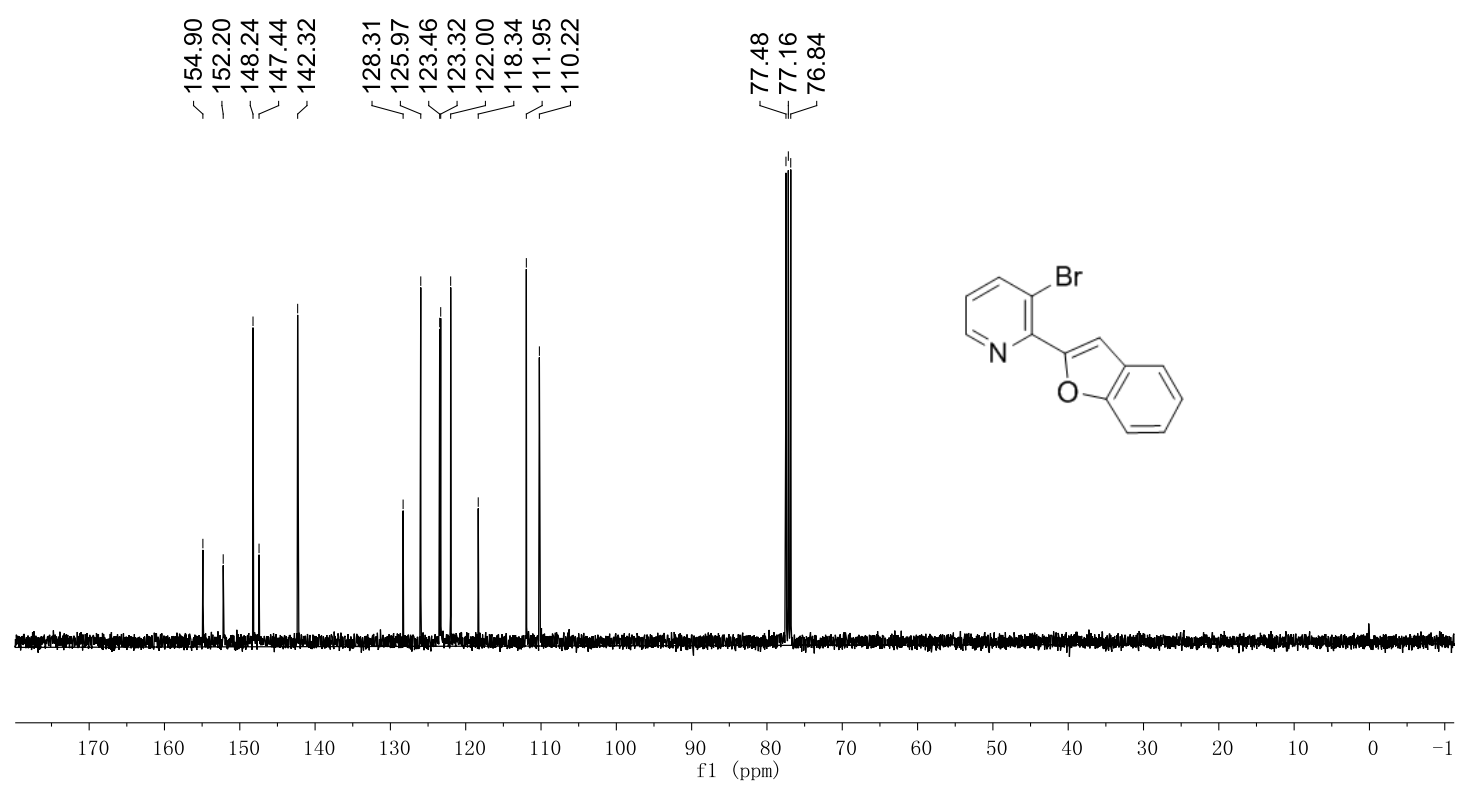

(12n)

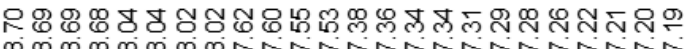
$\infty \infty \infty \infty \infty \infty \infty \pi N-N-N$
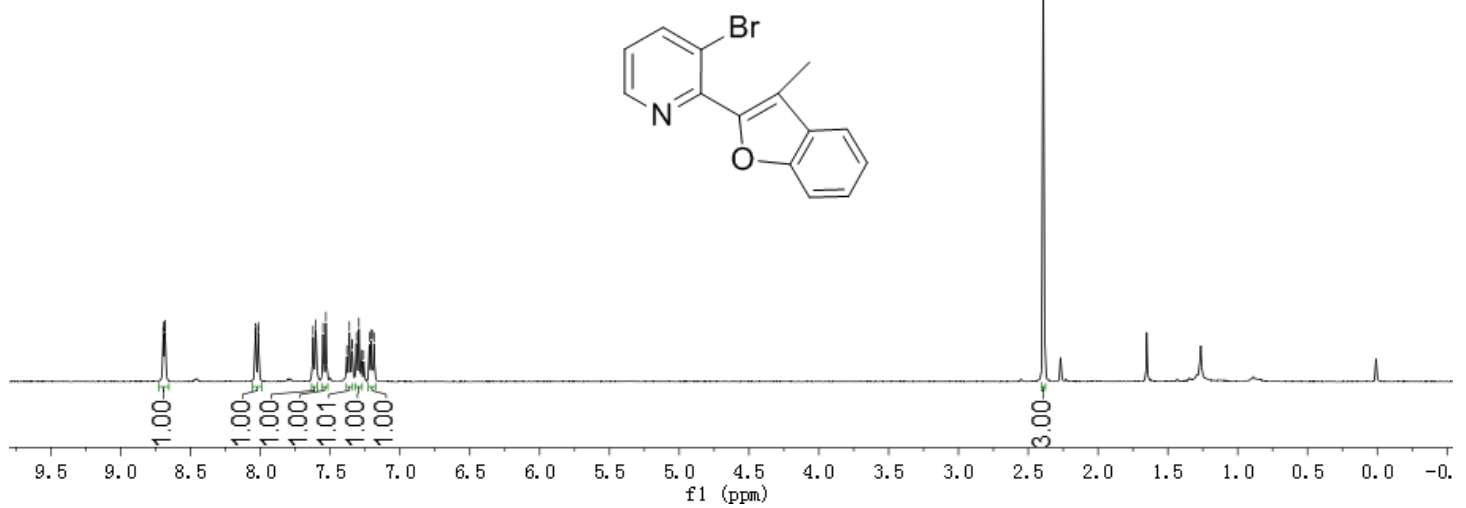


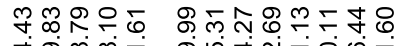

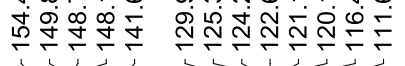

$\stackrel{\infty}{\sim} \underset{\sim}{\sim}$

Кำ

i̊․․

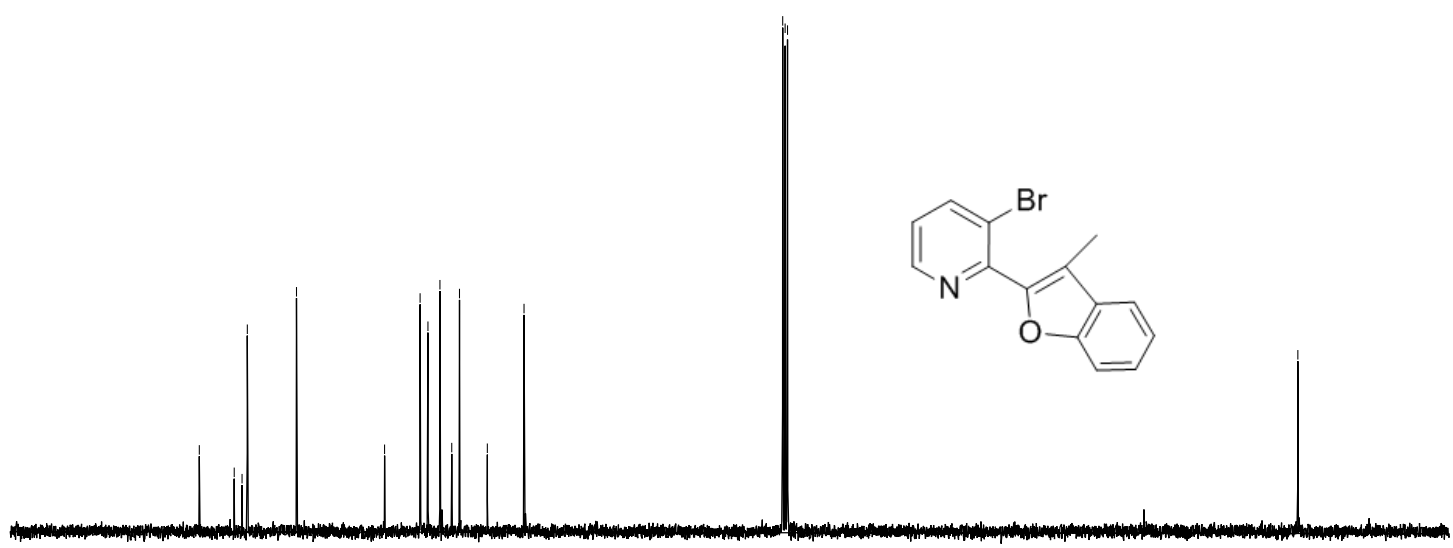

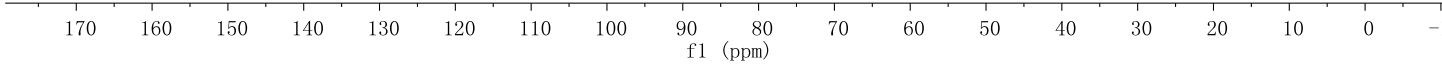

(13a)

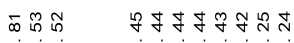

mon

영

넌도

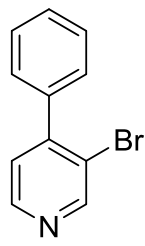

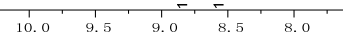

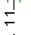


(13b)

ద

ion

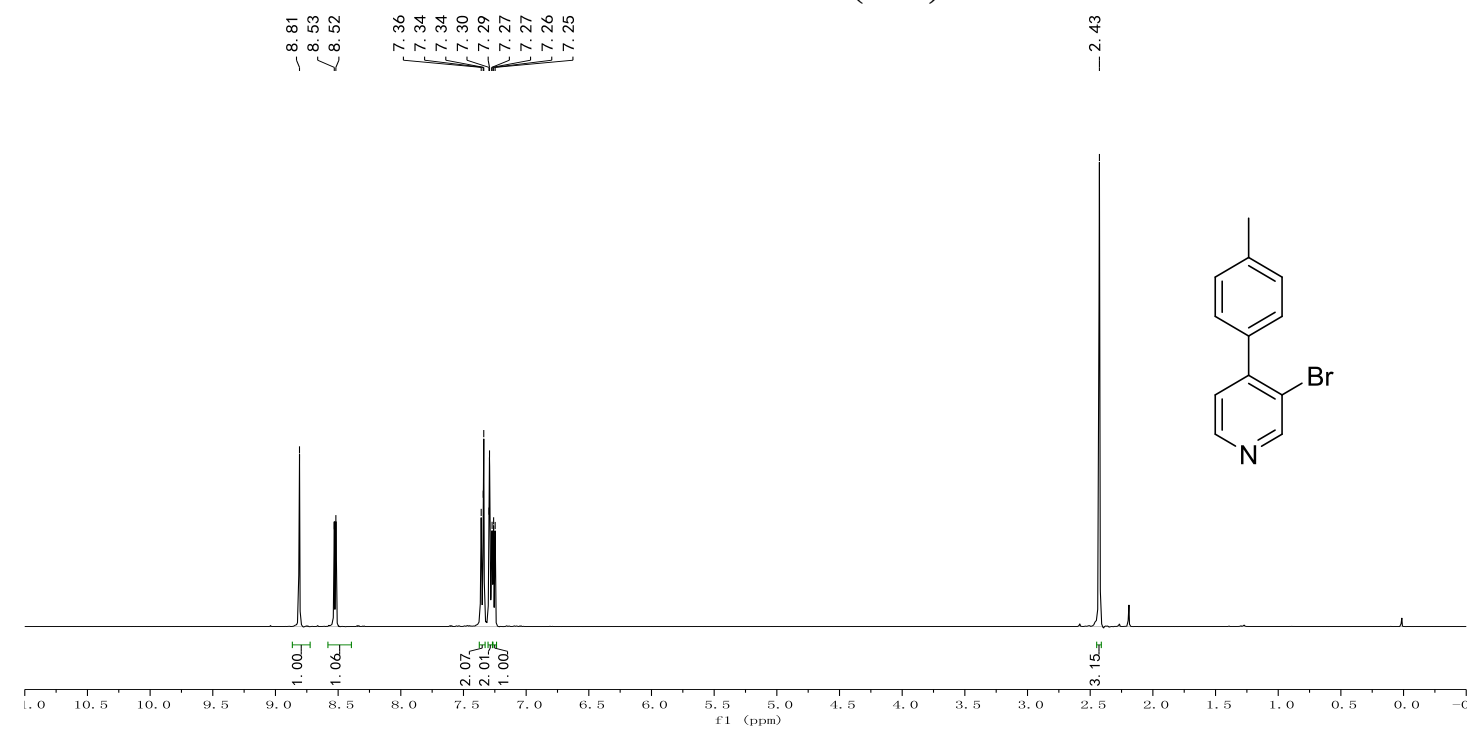

(13c)

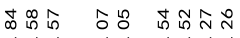

$\left.\bigcup^{\infty} \bigcup^{\infty}\right)^{\infty}$

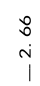

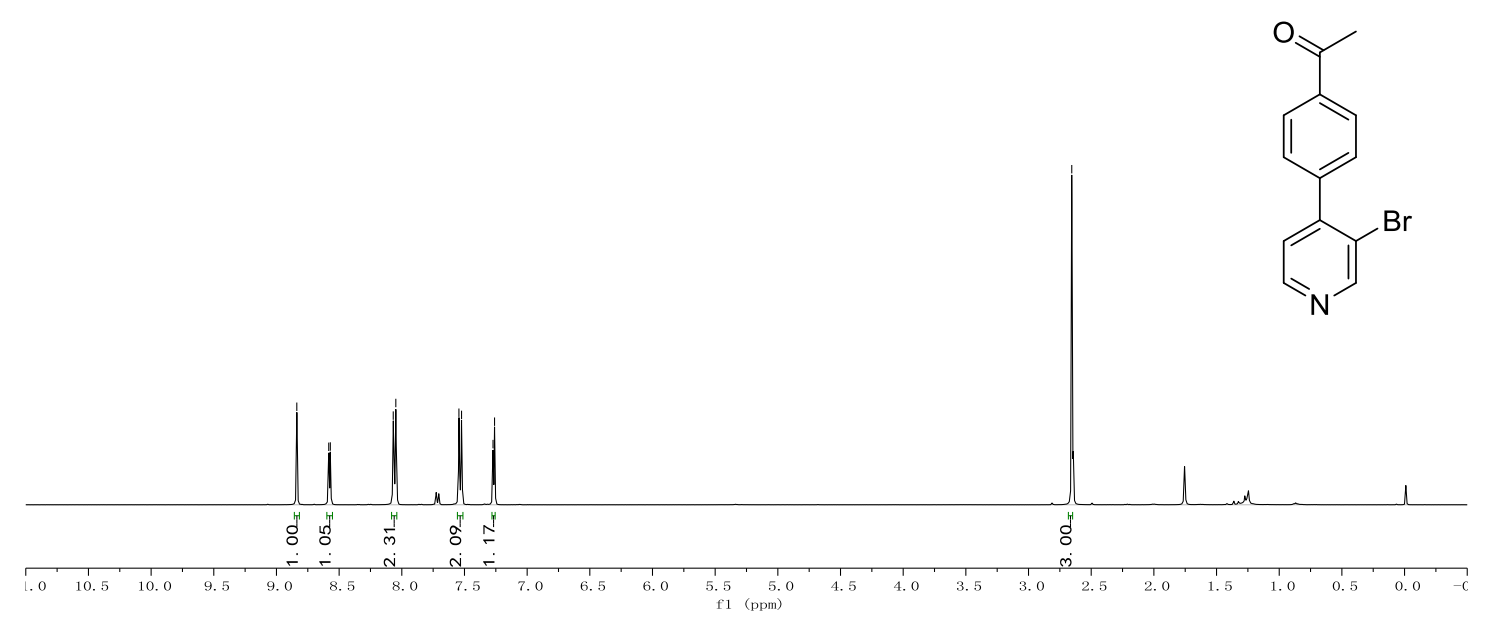



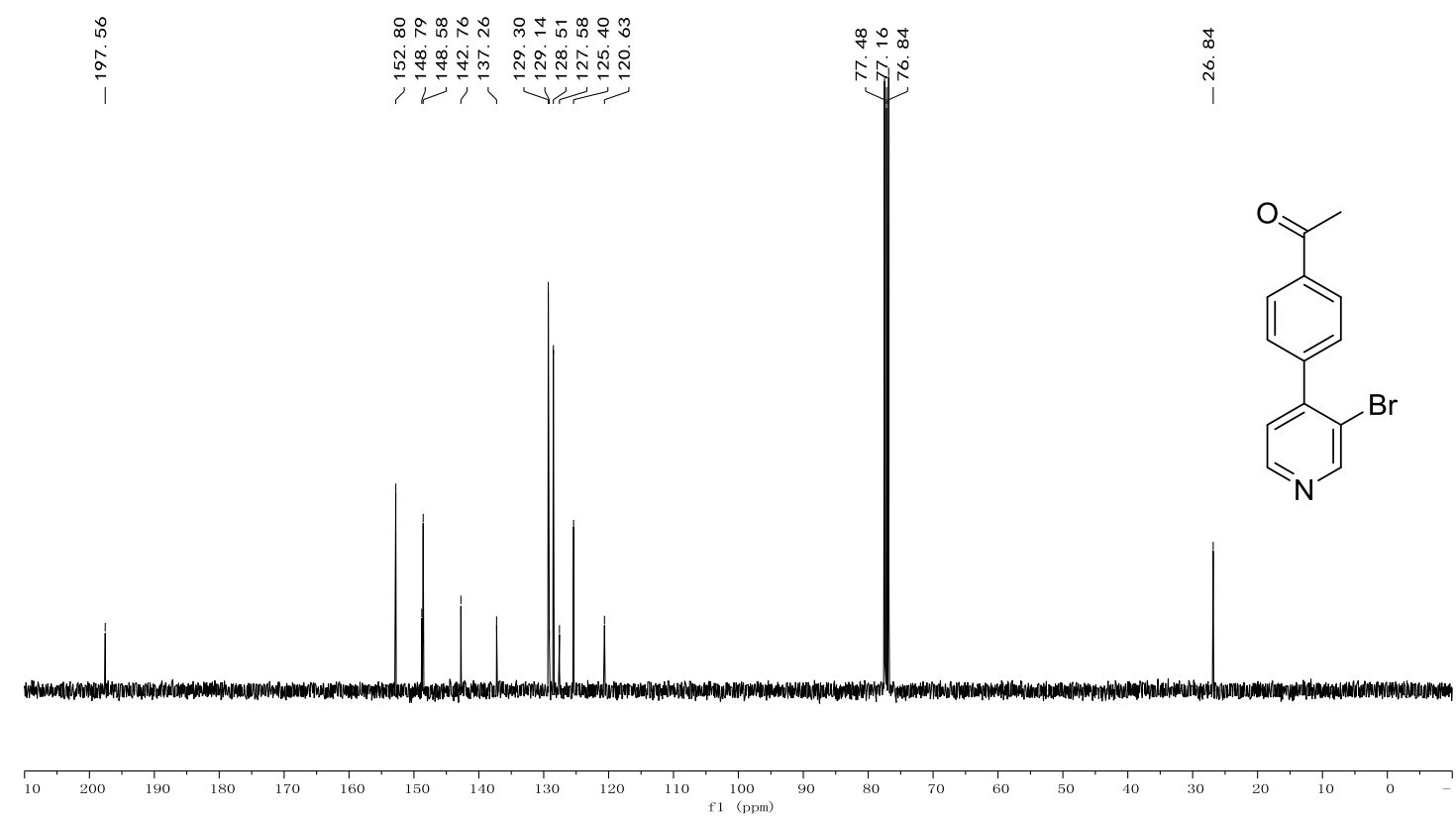

(14a)

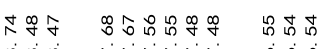

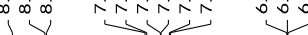

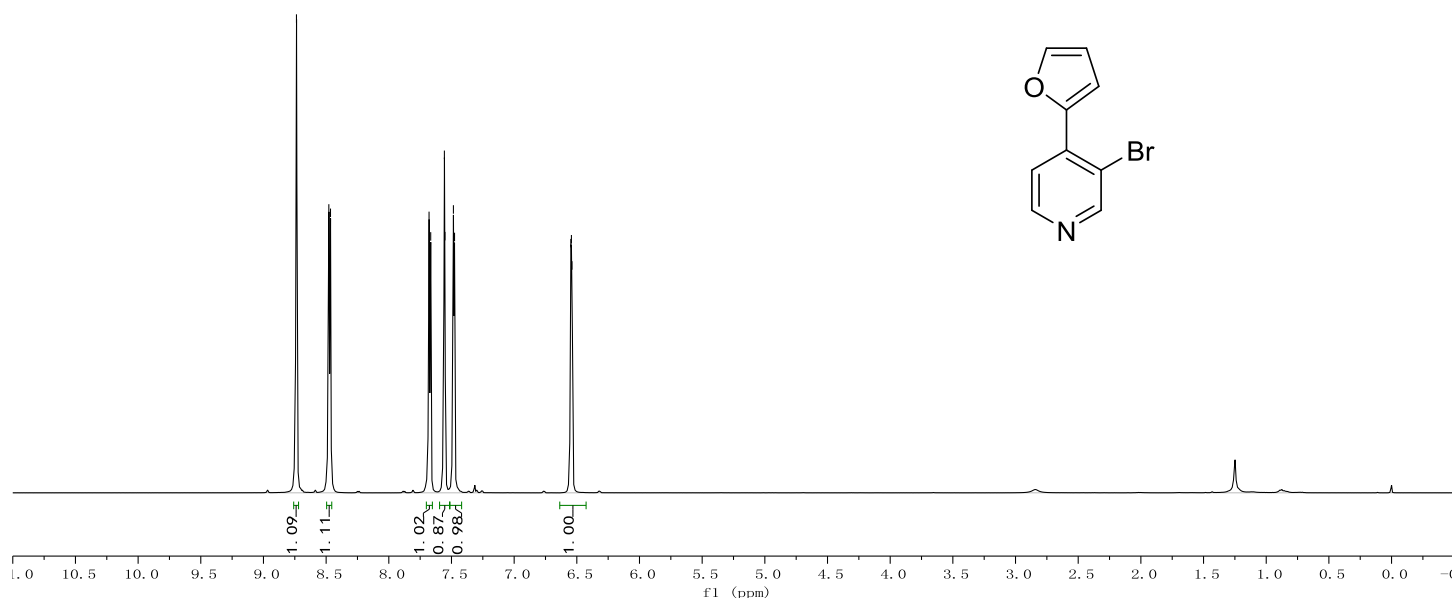




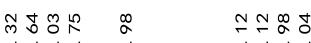

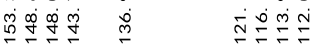

$\stackrel{+}{\sim} \stackrel{\infty}{\infty}$

एं। ए।

トล゚

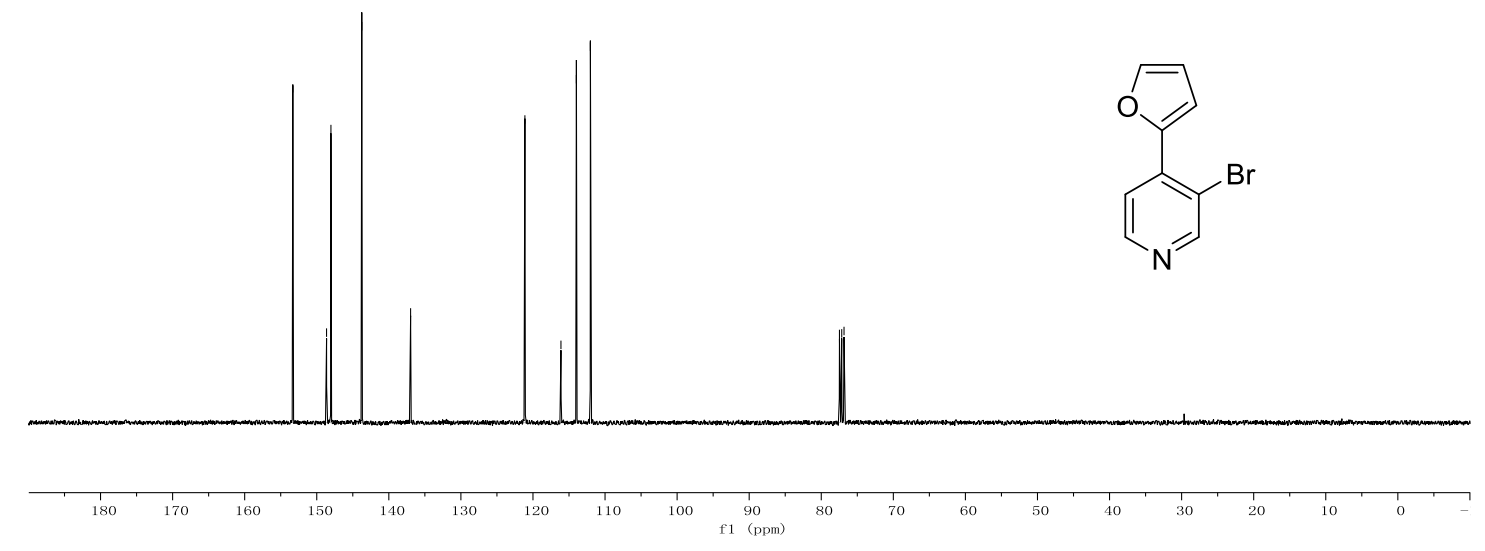

(14b)

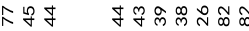

$\infty$ in risitio

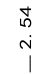

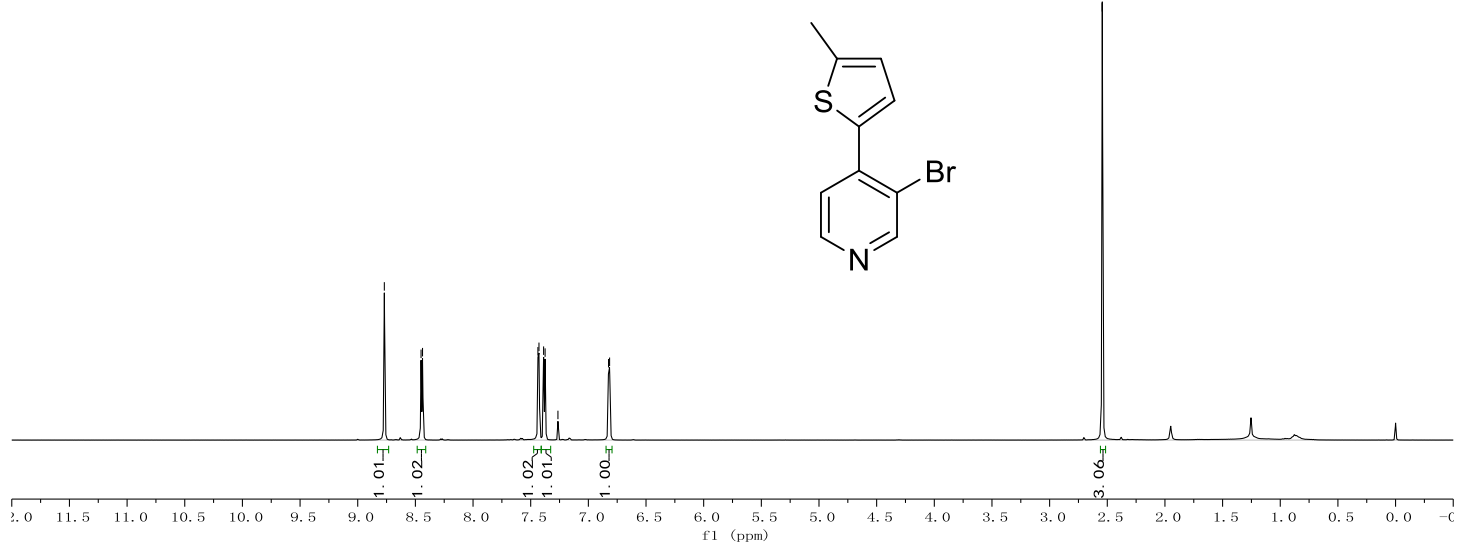



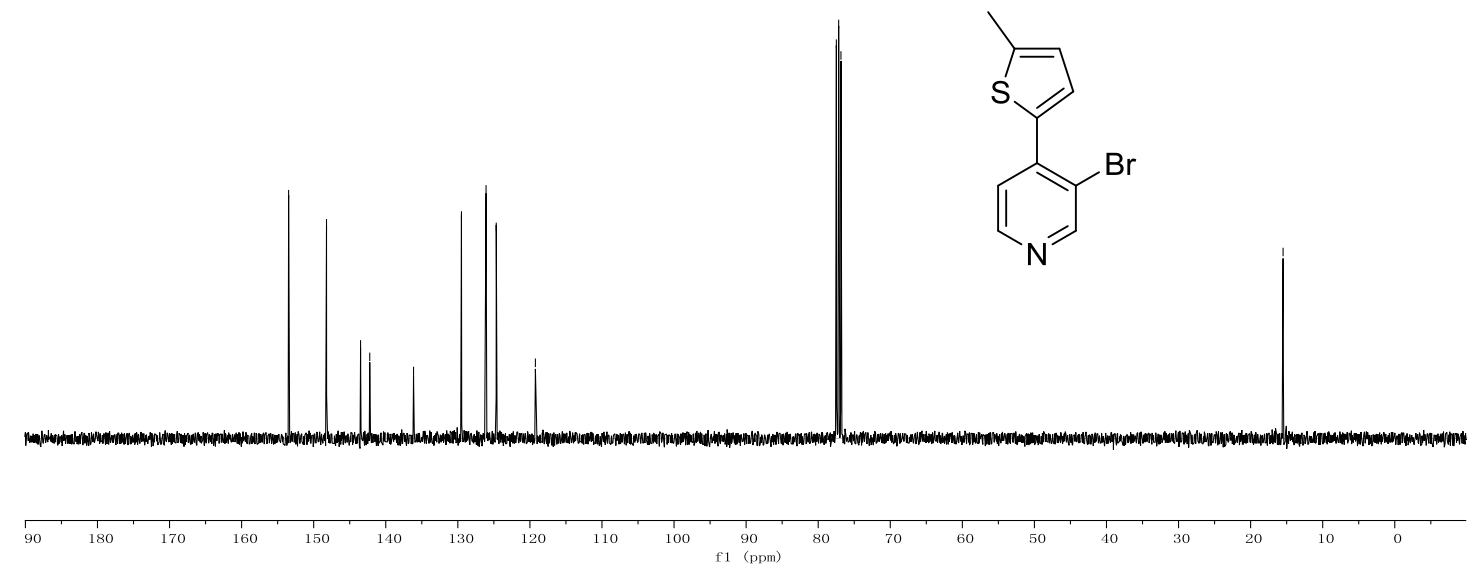

(14c)

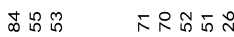

i ${ }^{\infty}$

0
กิ
ก
1

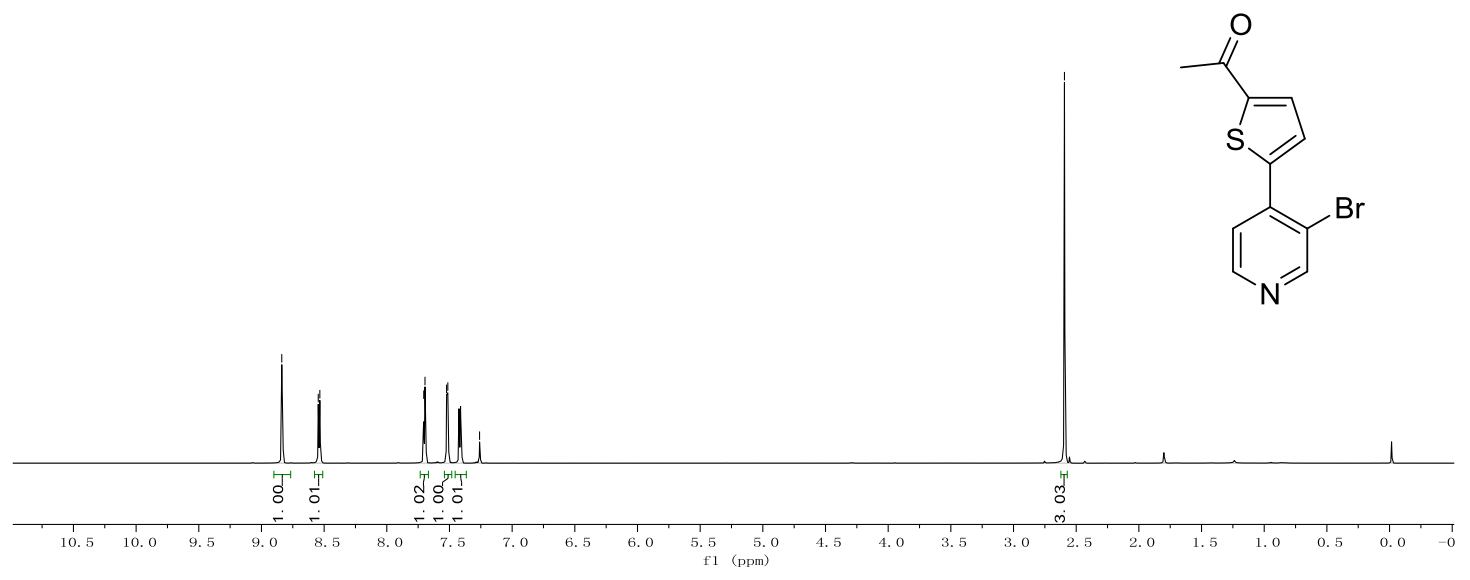




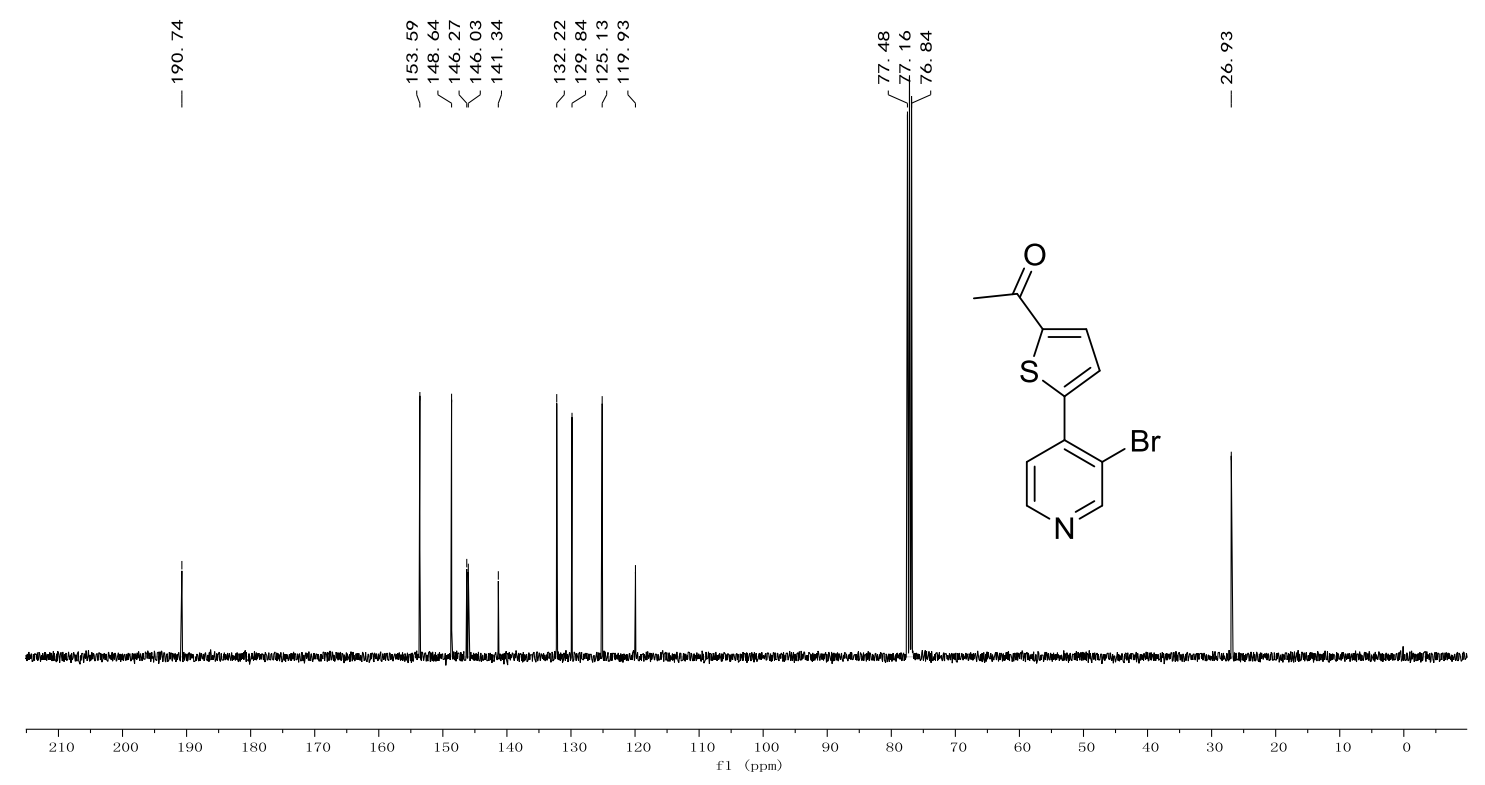

(14d)

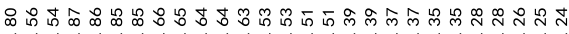

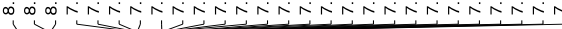

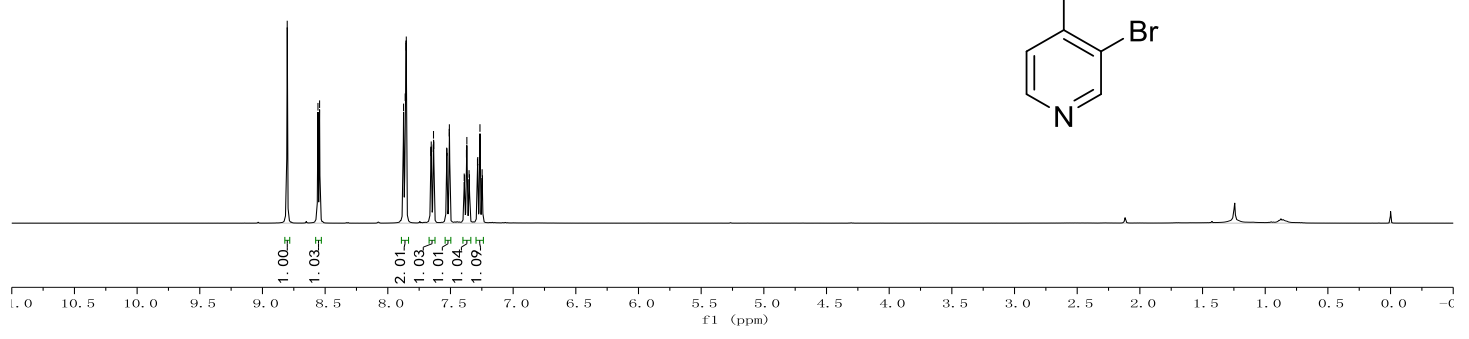




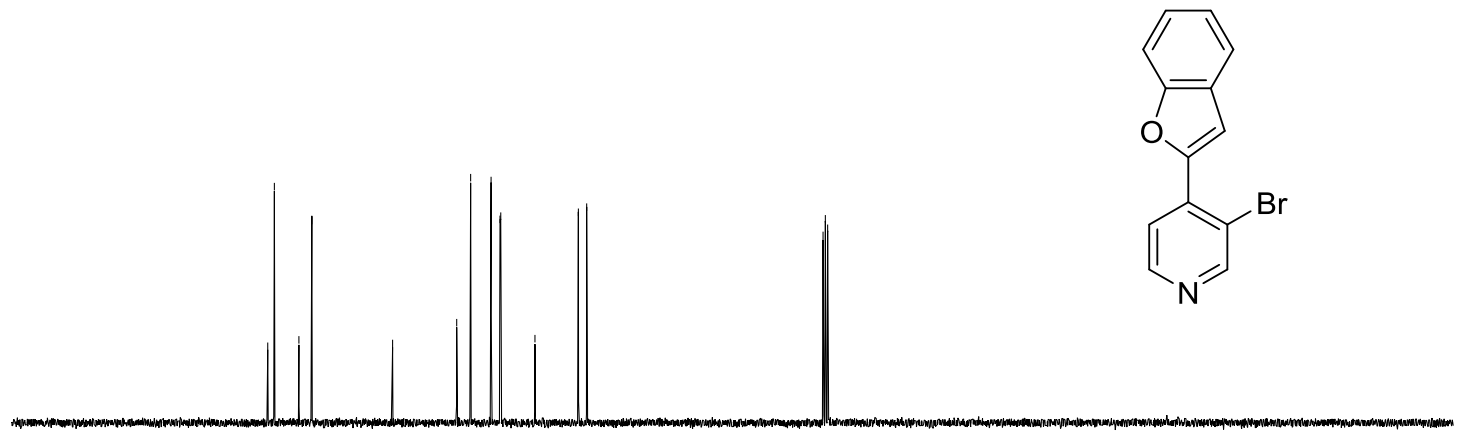


The spectra of 1a-1t, 3a-3d, 4a-4i, 7a-7d, 9a.

(1a)

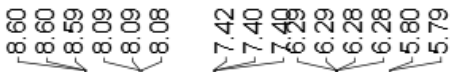

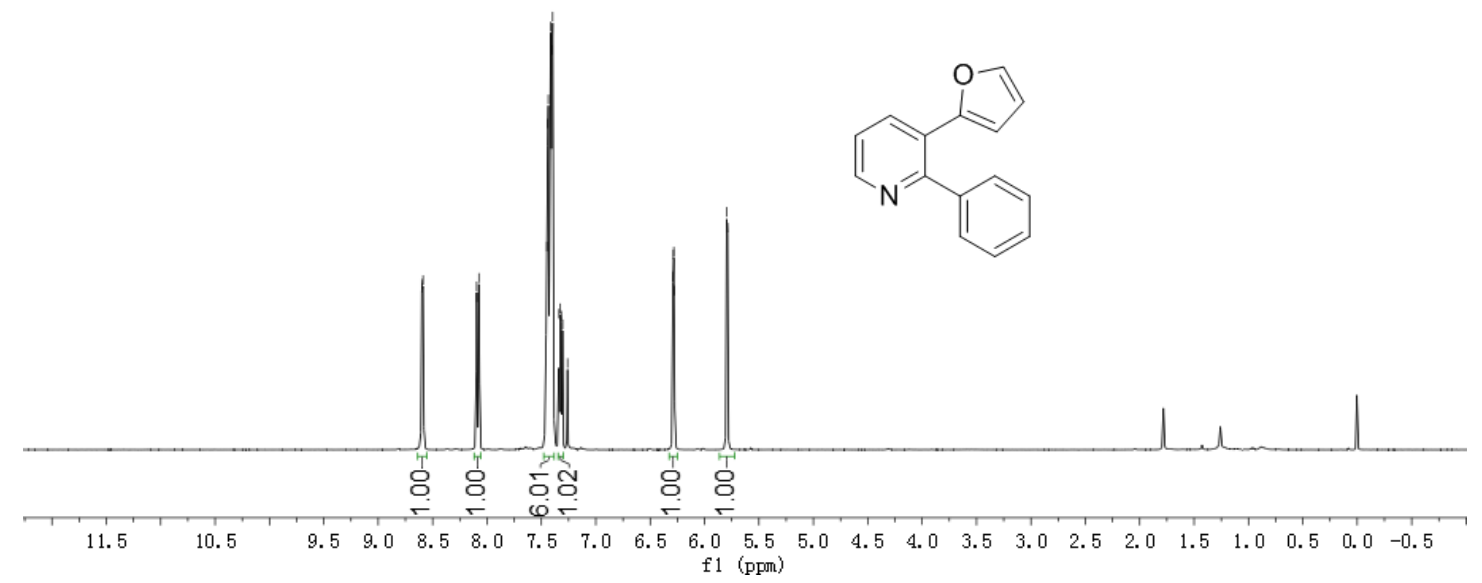

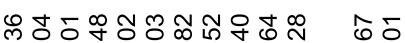

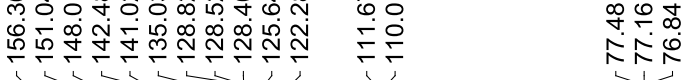

更

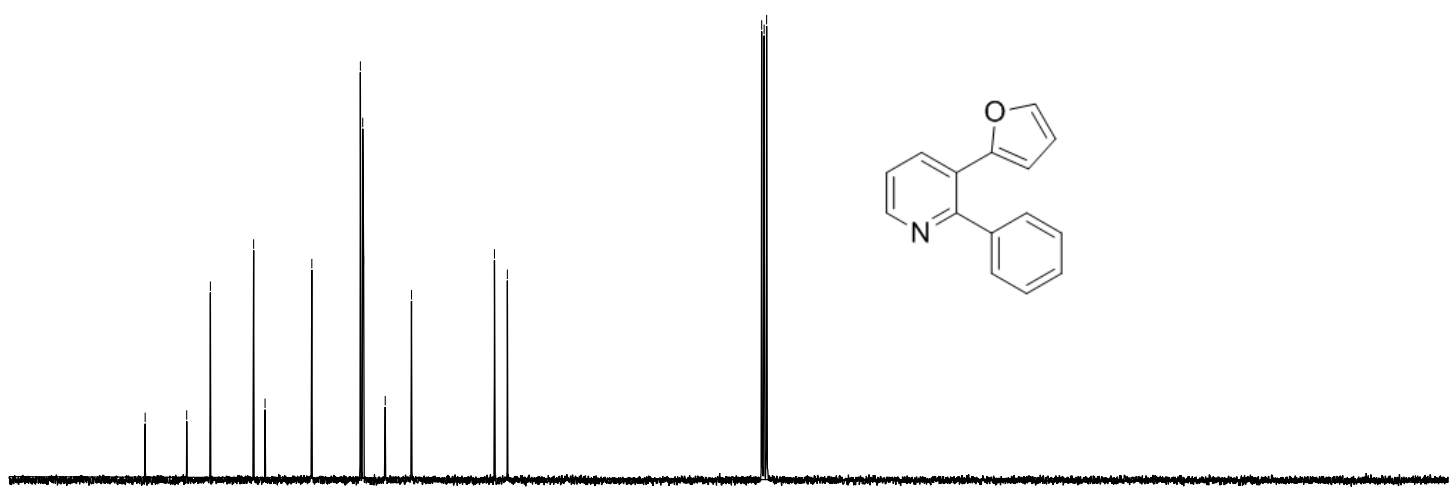

$\begin{array}{llllllllll}170 & 160 & 150 & 140 & 130 & 120 & 110 & 100 & 90 & 80\end{array}$

f1 (ppm) 
(1b)

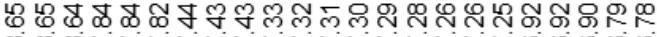

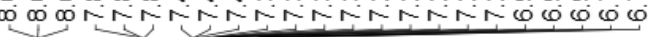

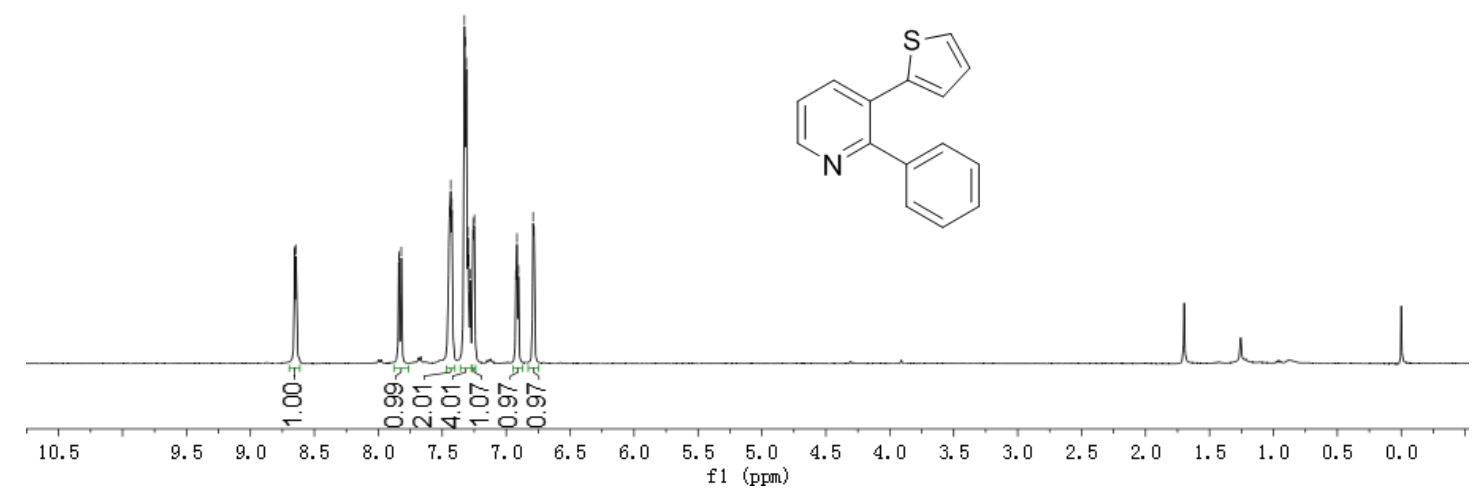

๑ ஜ

เి
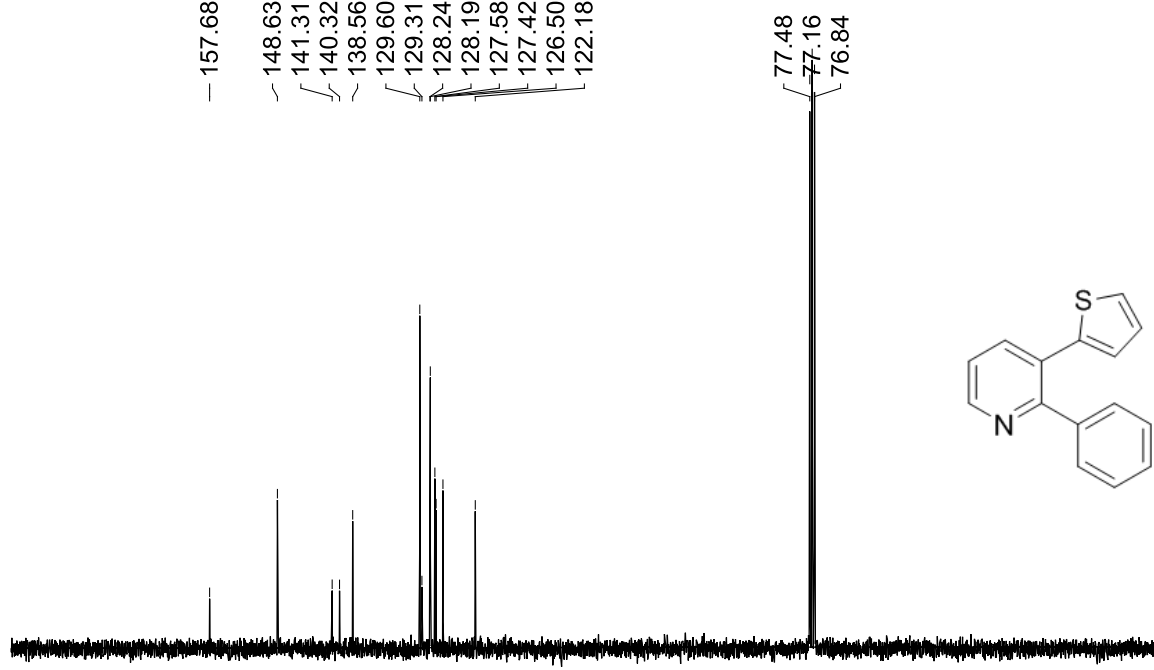

$180 \quad 170$

$120 \quad 110 \quad 100$

${ }^{90}(\mathrm{ppm})$ 
(1c)

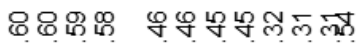

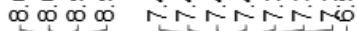

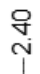

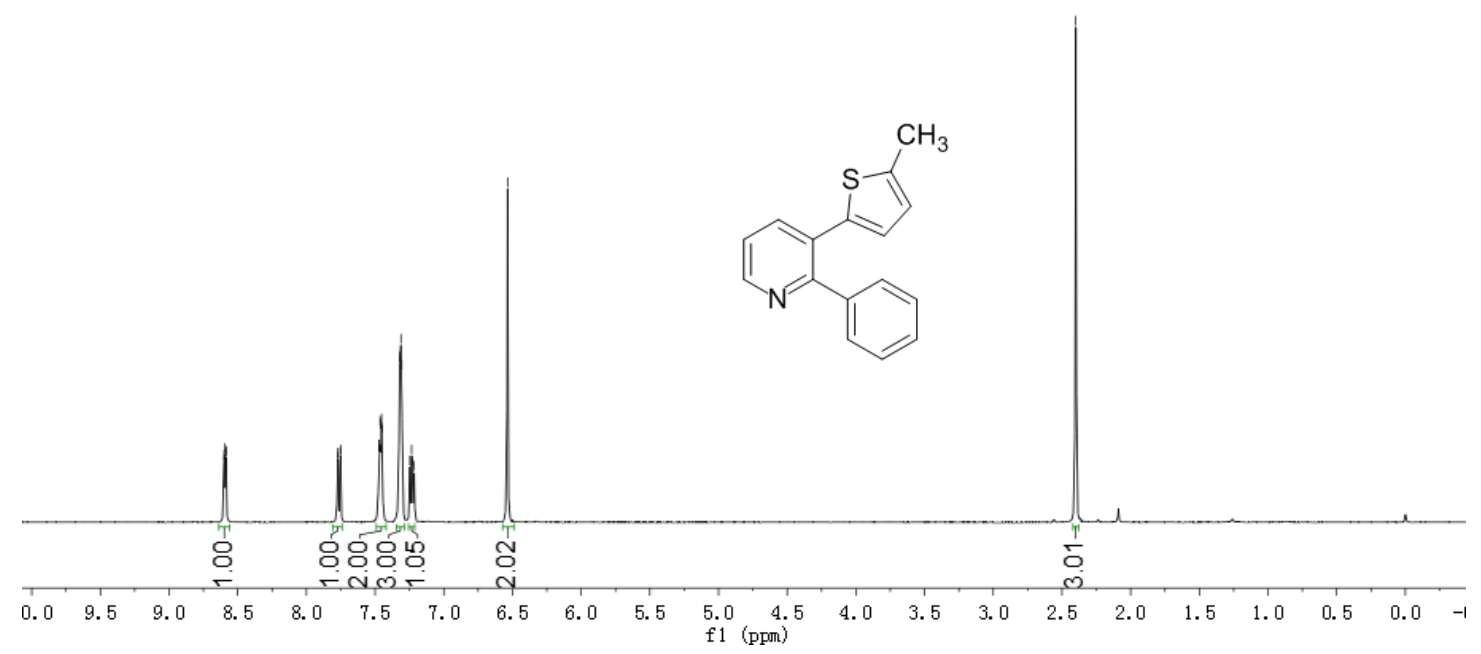

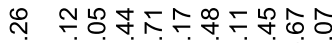

它

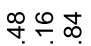

रำ

๗ำ

供

$\frac{1}{1}$

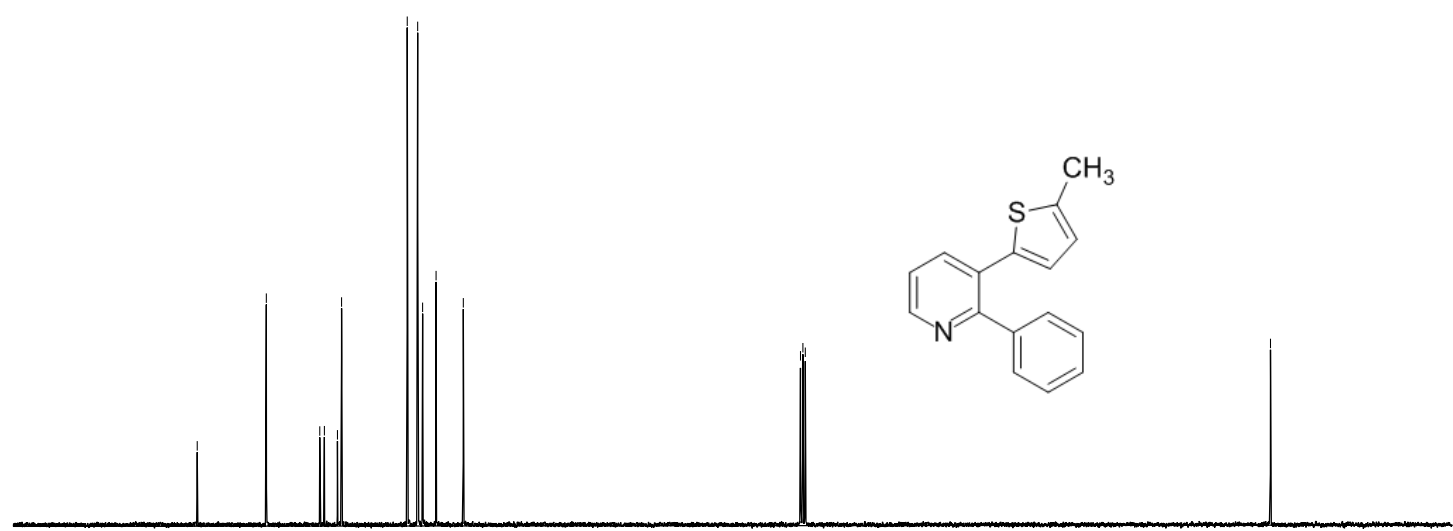

$180 \quad 17$

$\begin{array}{llllllll}160 & 150 & 140 & 130 & 120 & 110 & 100 & \begin{array}{c}90 \\ \mathrm{f} 1(\mathrm{ppm})\end{array}\end{array}$ 
(1d)

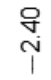
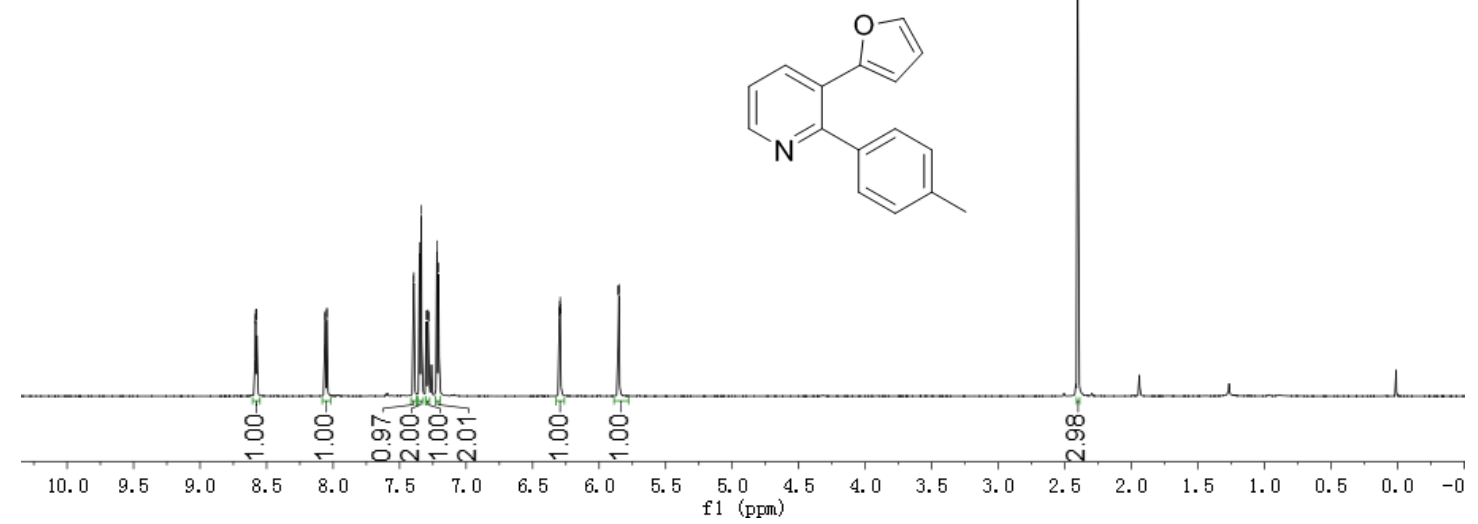

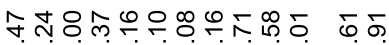

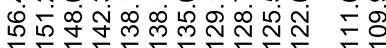

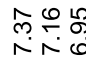

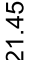

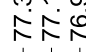

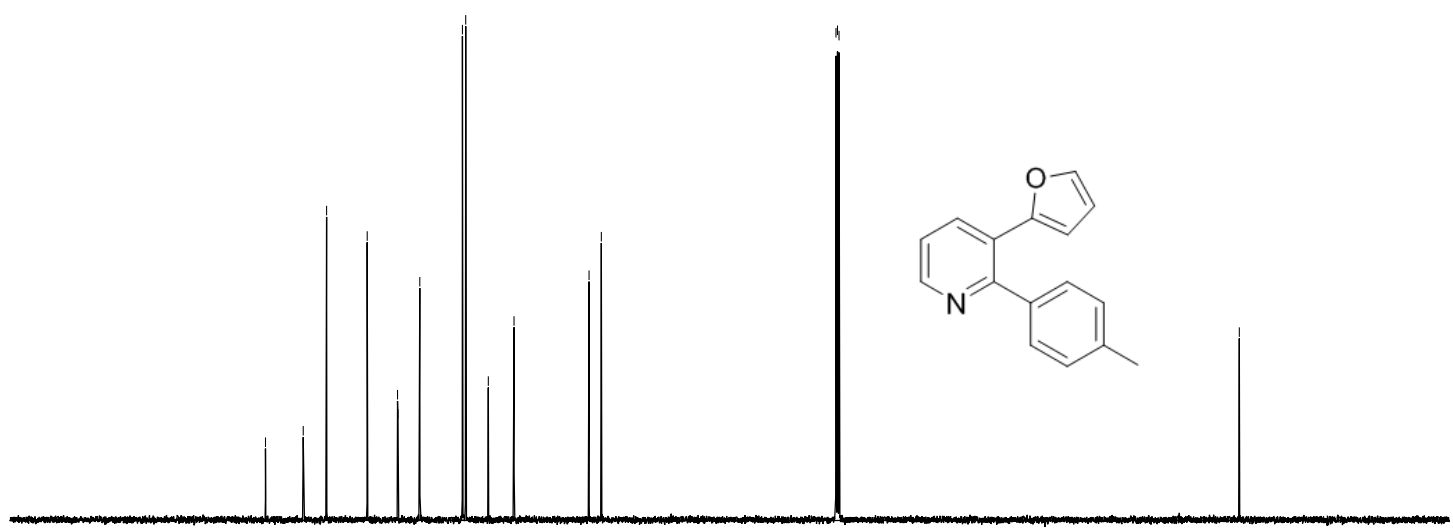

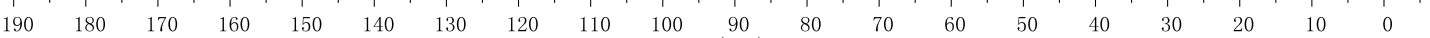




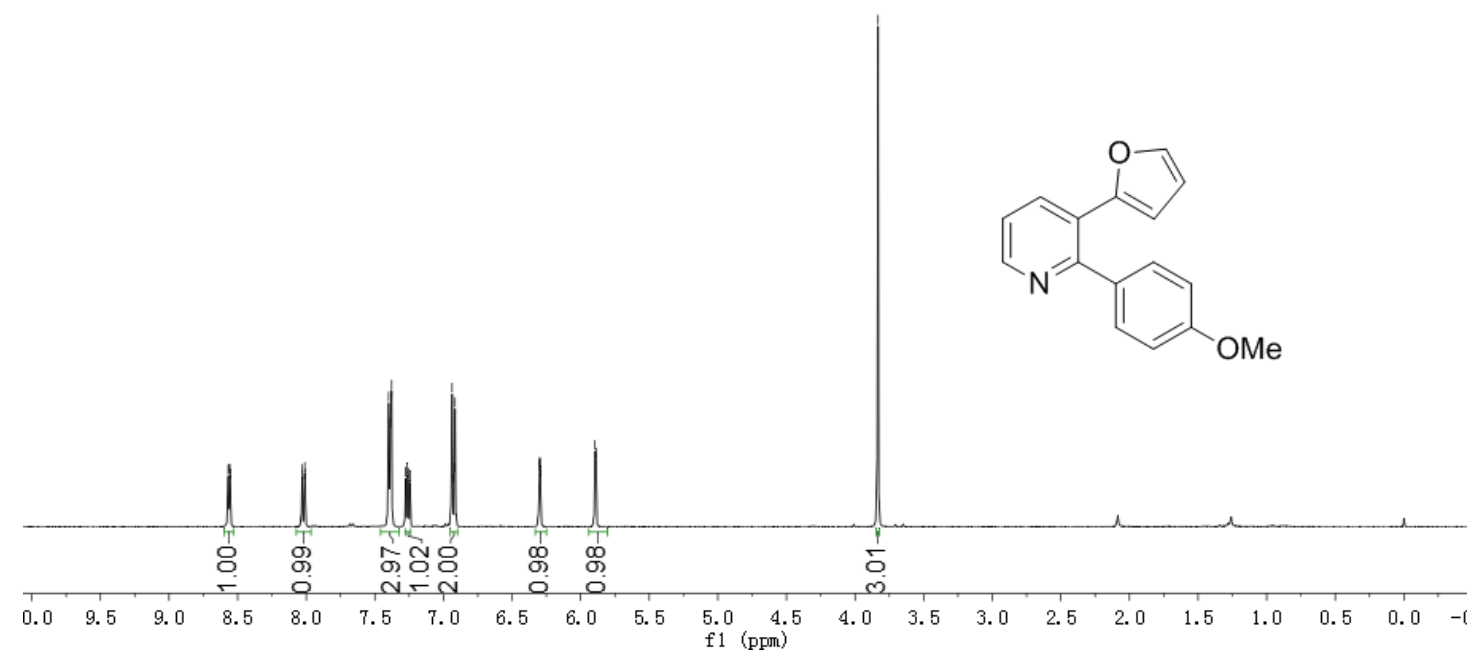

ธ్రం

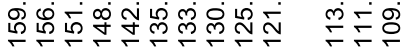

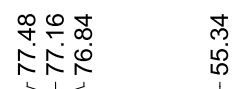

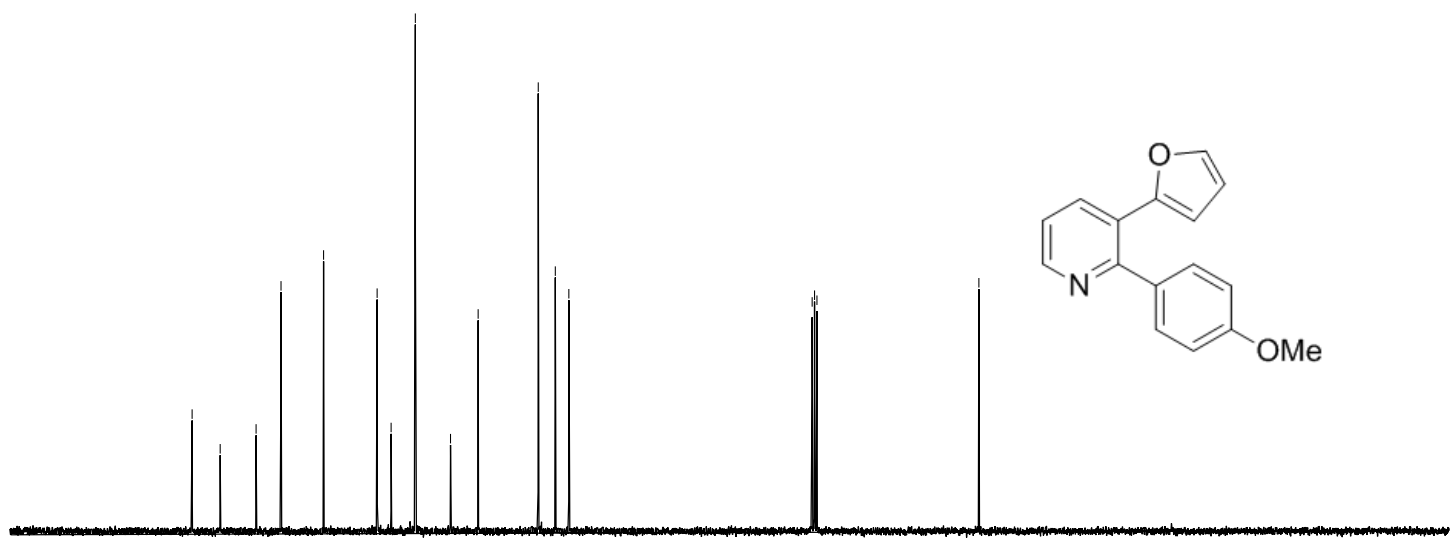

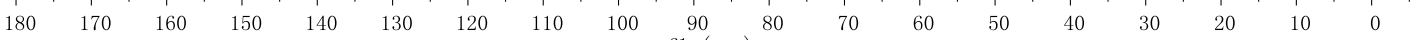




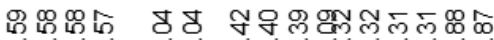

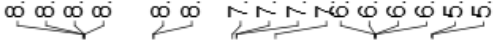
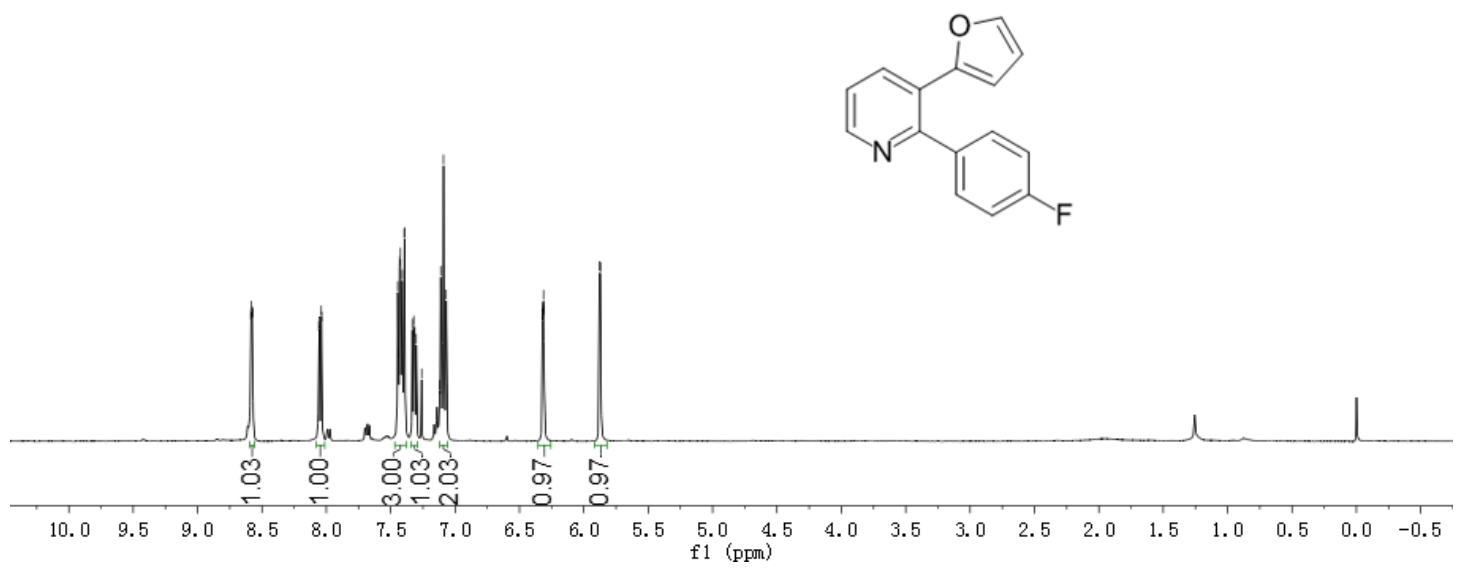

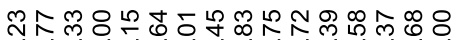

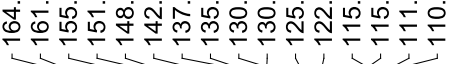

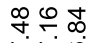

송

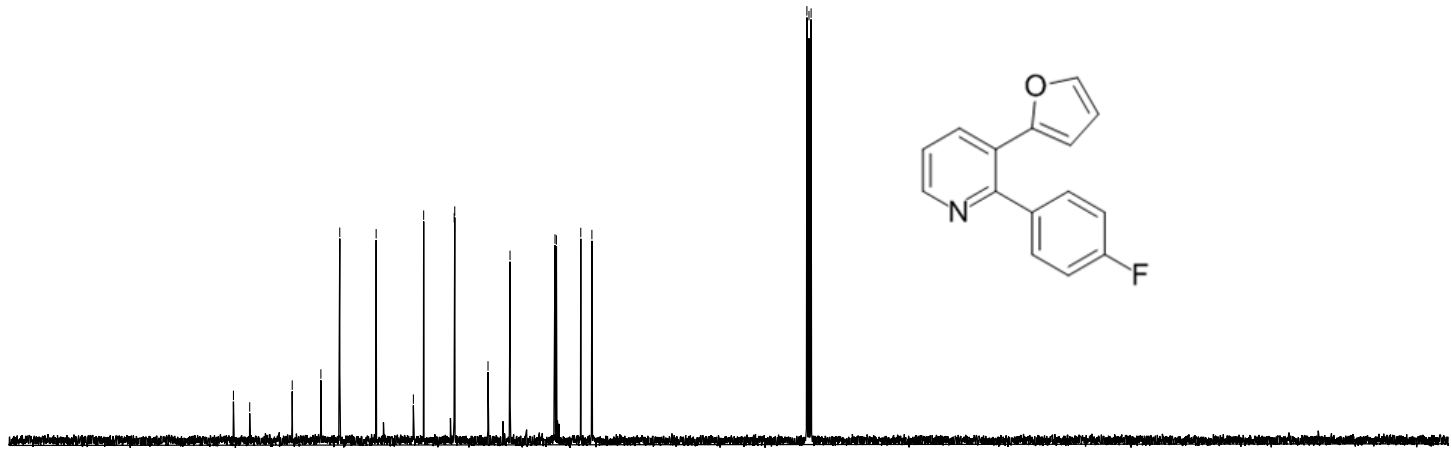

$\begin{array}{lllllllllll}190 & 180 & 170 & 160 & 150 & 140 & 130 & 120 & 110 & 100 & \underset{\mathrm{f} 1}{90}(\mathrm{ppm})\end{array}$ 
(1g)

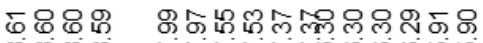

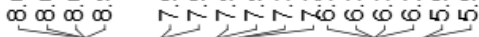

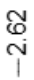

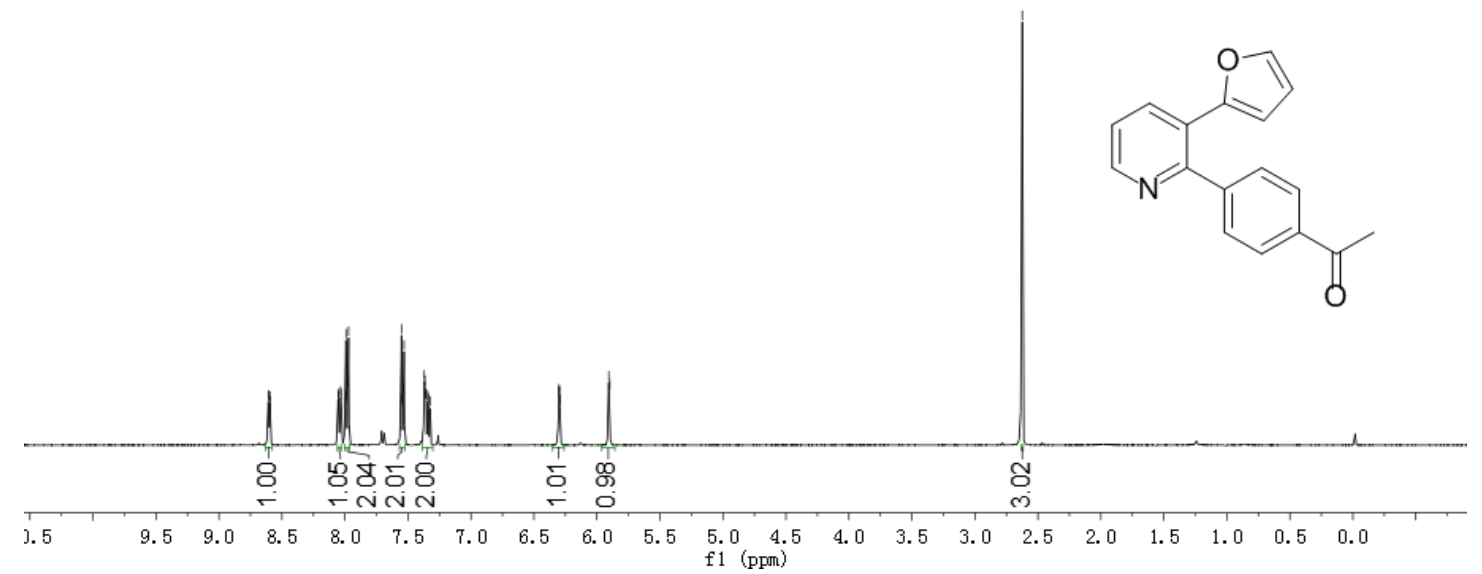

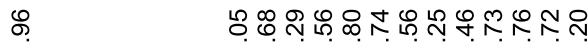

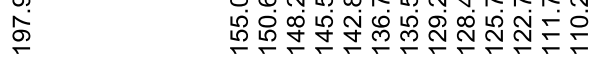

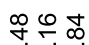

ヘト゚゚

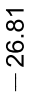

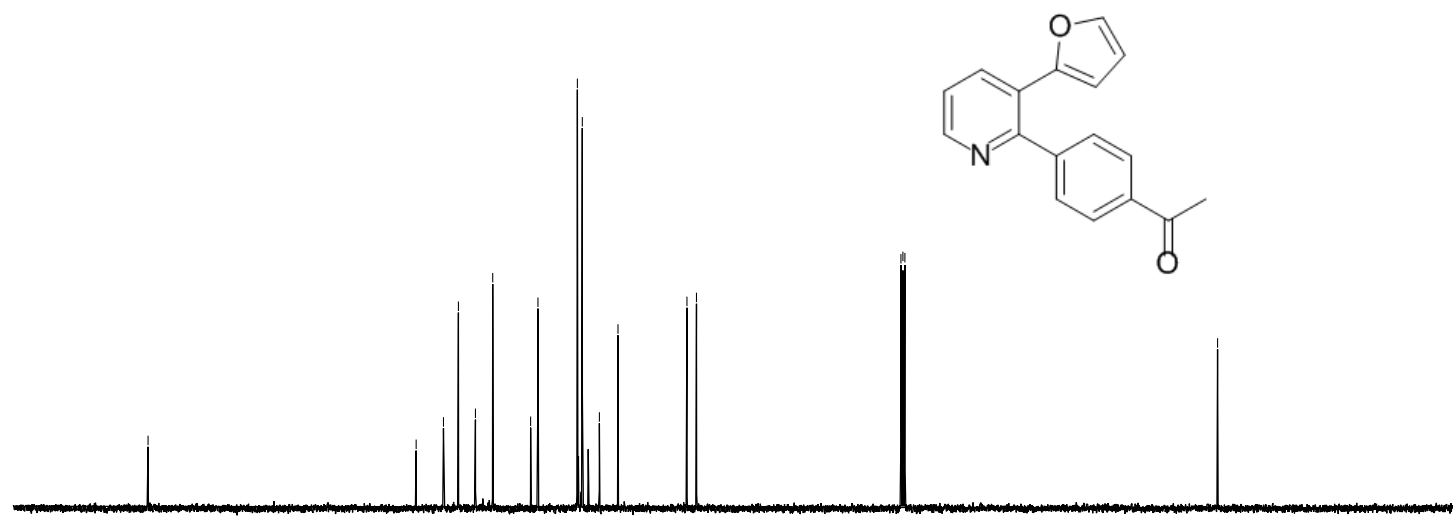

$\begin{array}{llllllllllll}210 & 200 & 190 & 180 & 170 & 160 & 150 & 140 & 130 & 120 & 110 & 1\end{array}$ 


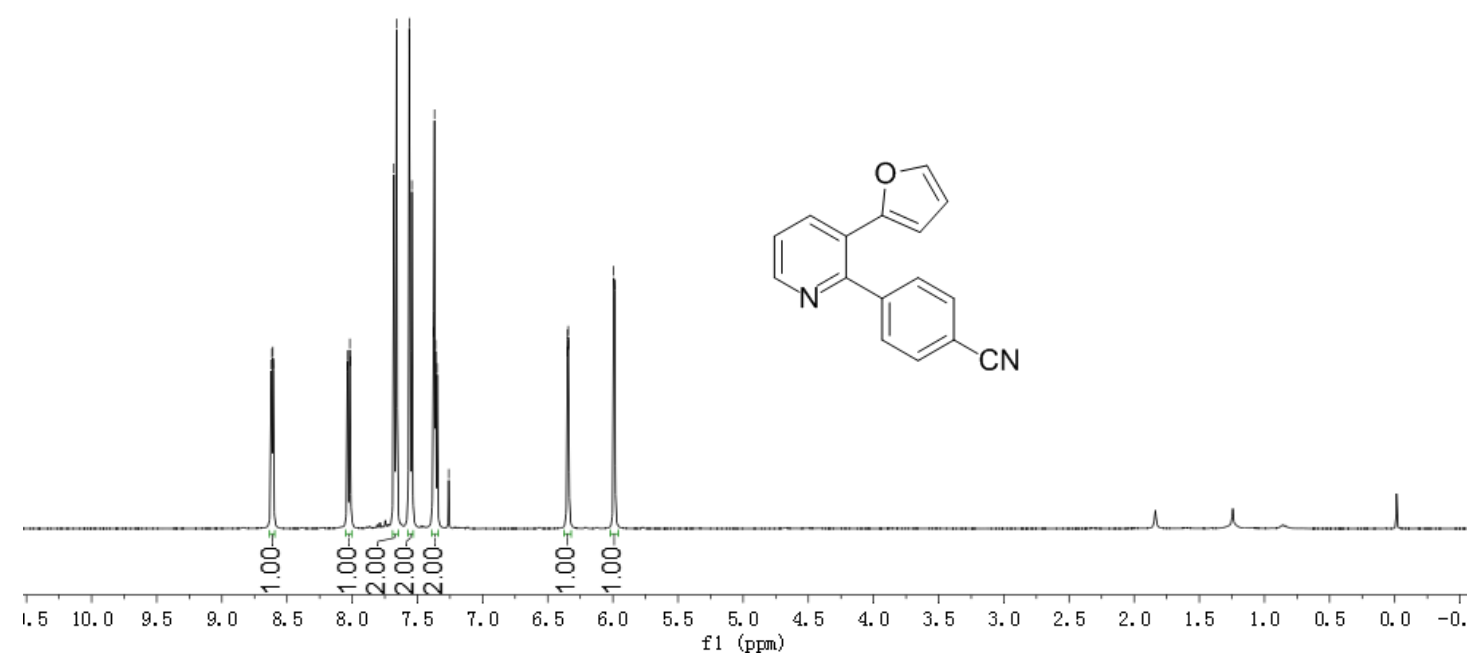

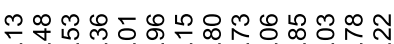

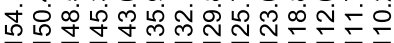

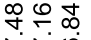

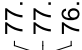

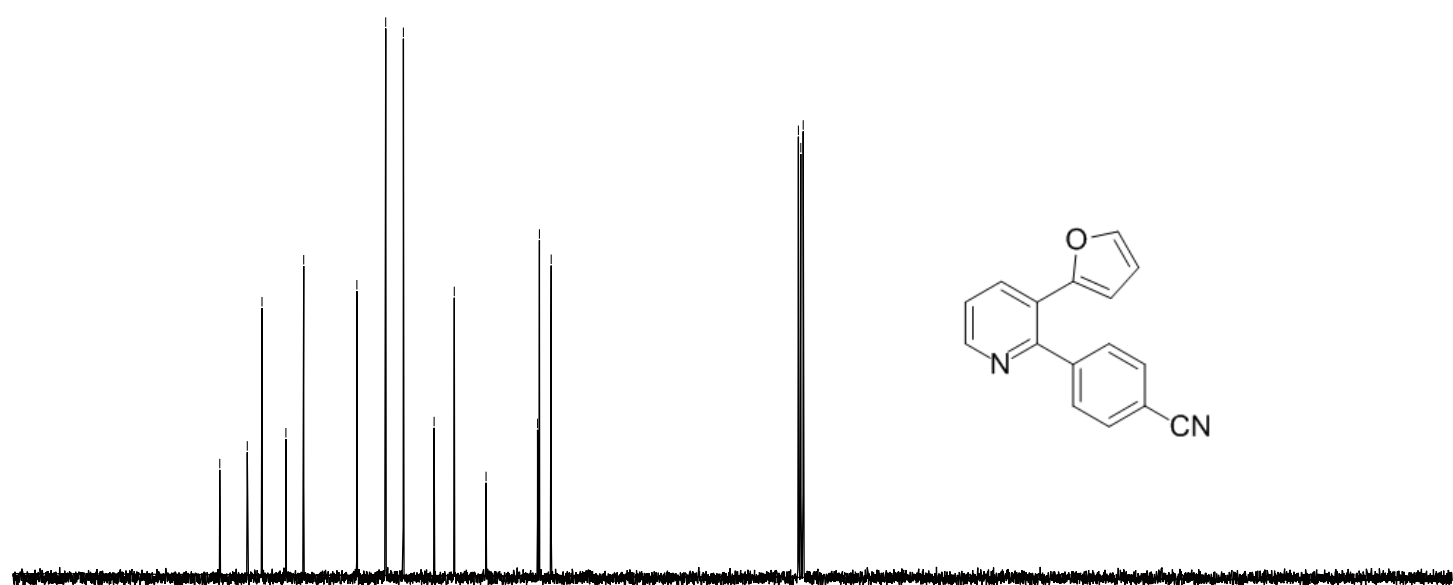

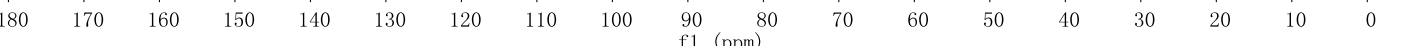




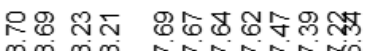

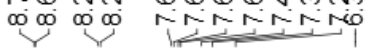

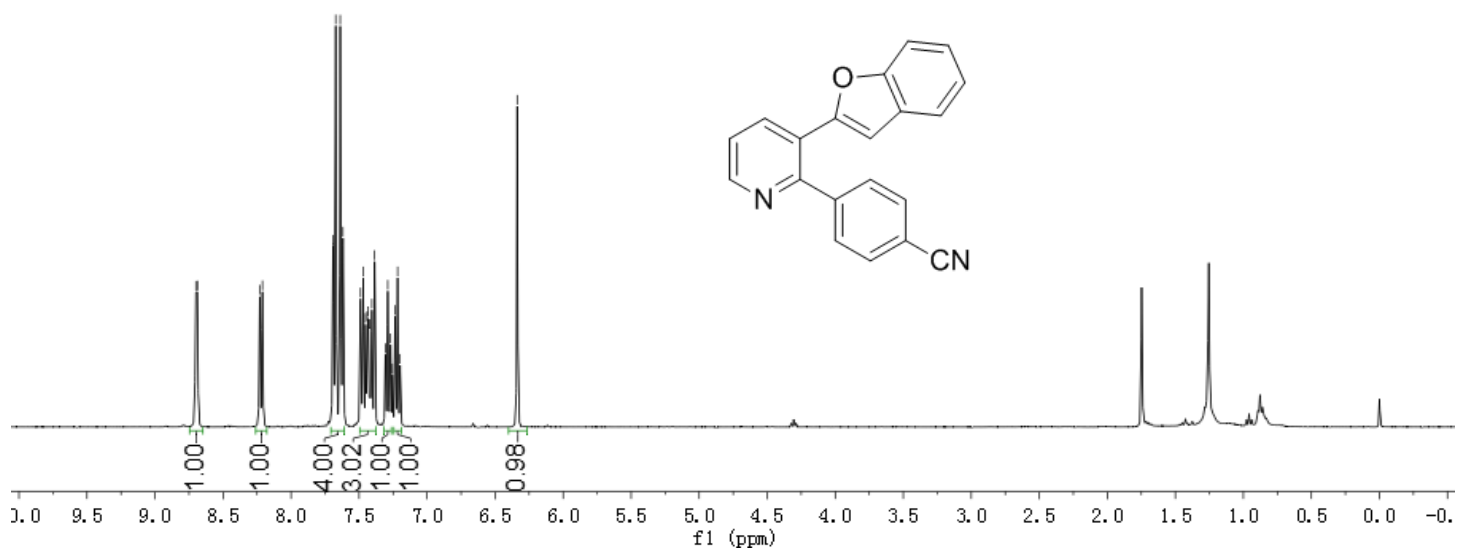

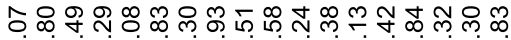

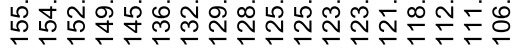

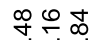

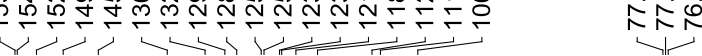

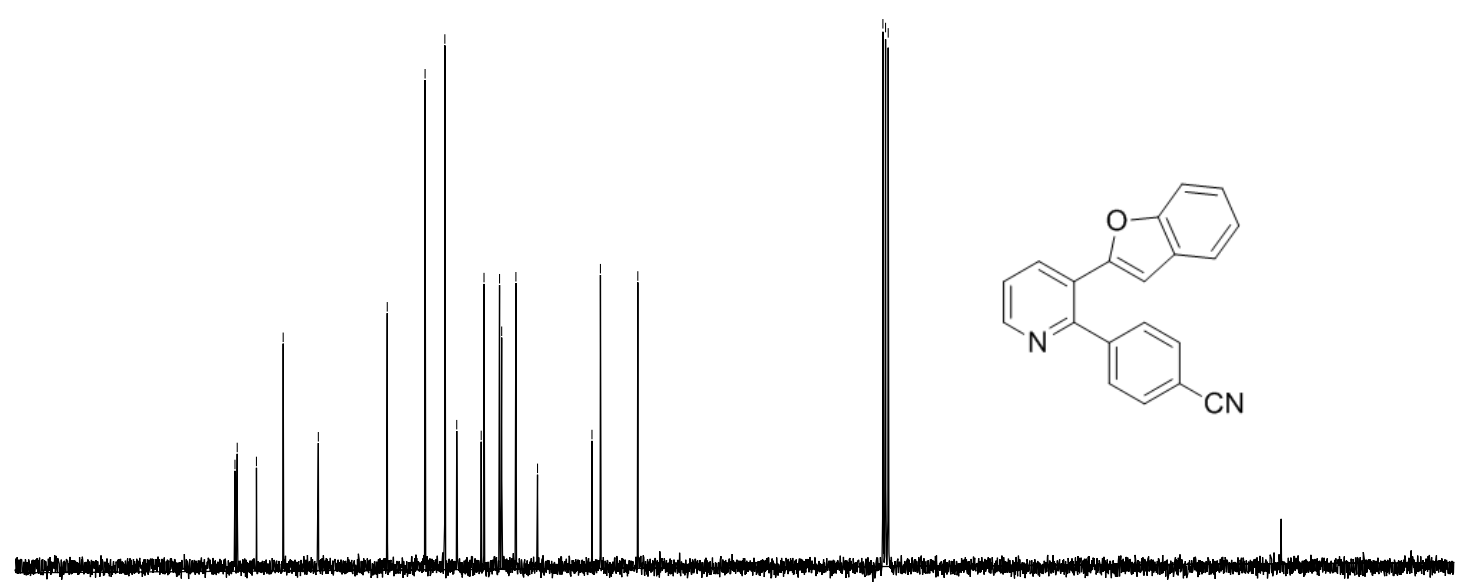

$\begin{array}{llllllllllllllllll}180 & 170 & 160 & 150 & 140 & 130 & 120 & 110 & \begin{array}{c}100 \\ \mathrm{f} 1(\mathrm{pmm})\end{array} & 80 & 70 & 60 & 50 & 40 & 30 & 20 & 1\end{array}$ 

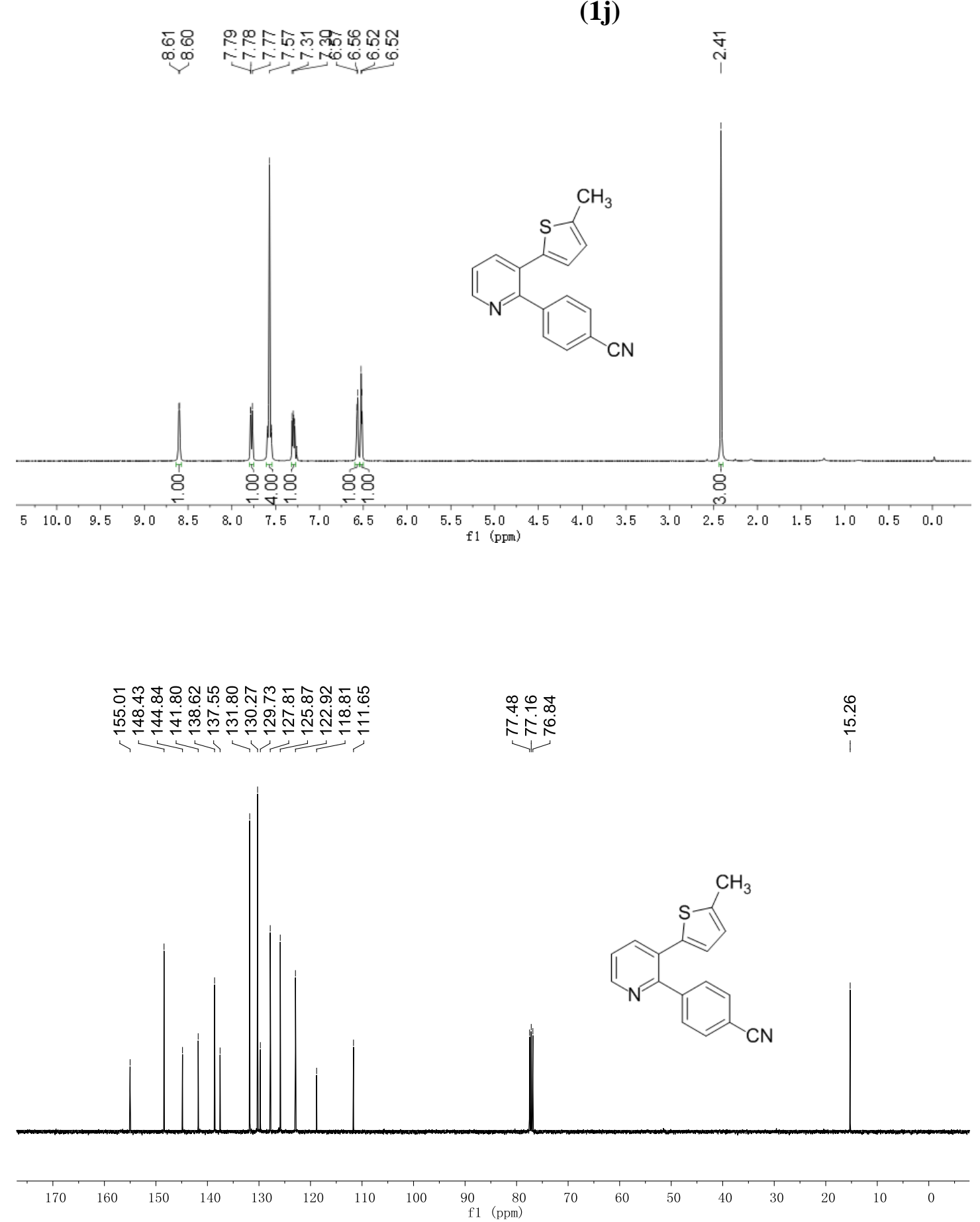


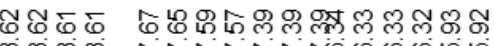

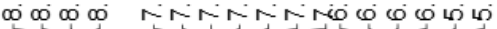
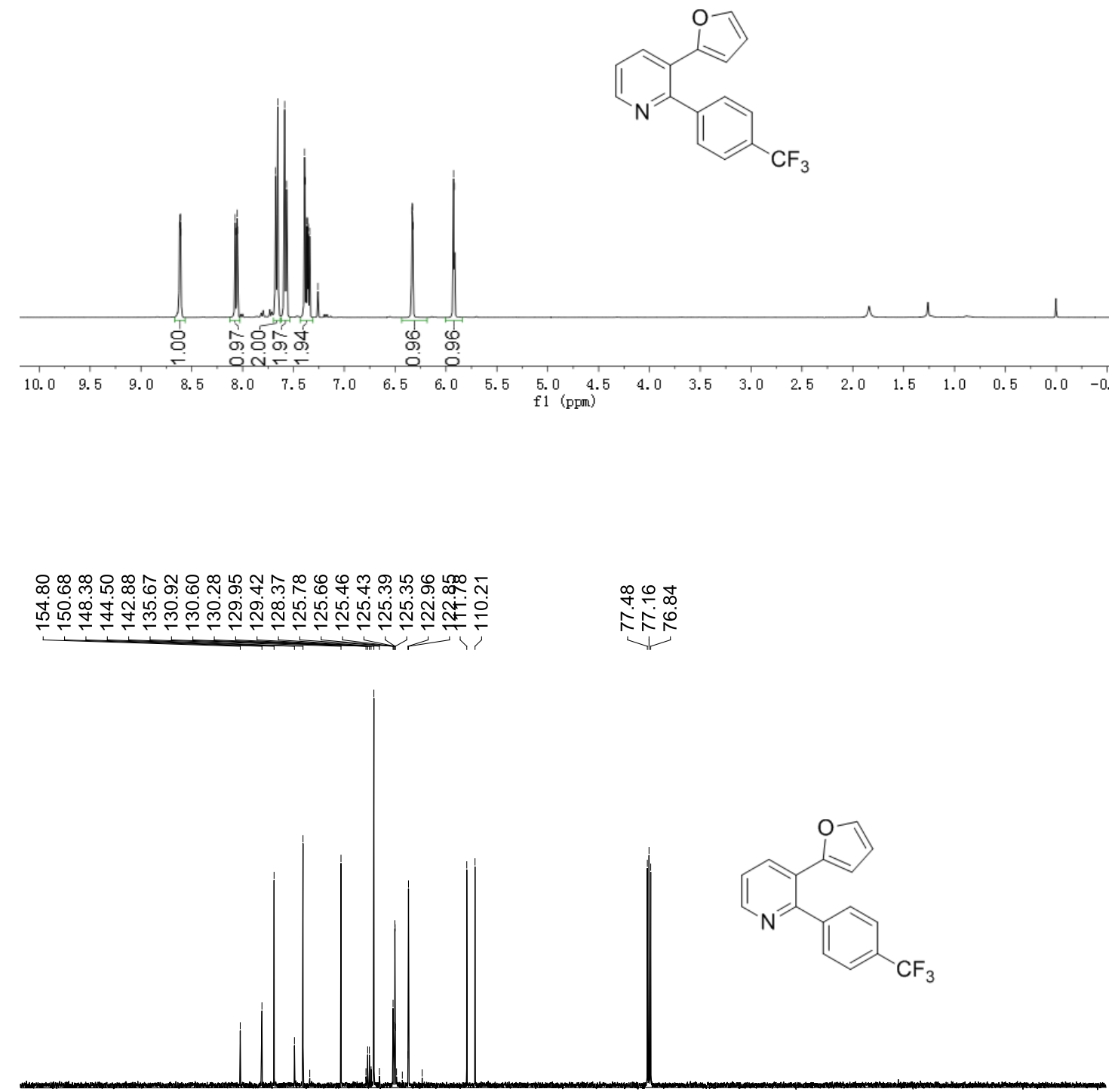

$\begin{array}{rrrrrrrrrrr}190 & 180 & 170 & 160 & 150 & 140 & 130 & 120 & 110 & 100 & \begin{array}{r}90 \\ \text { fpm })\end{array}\end{array}$ 

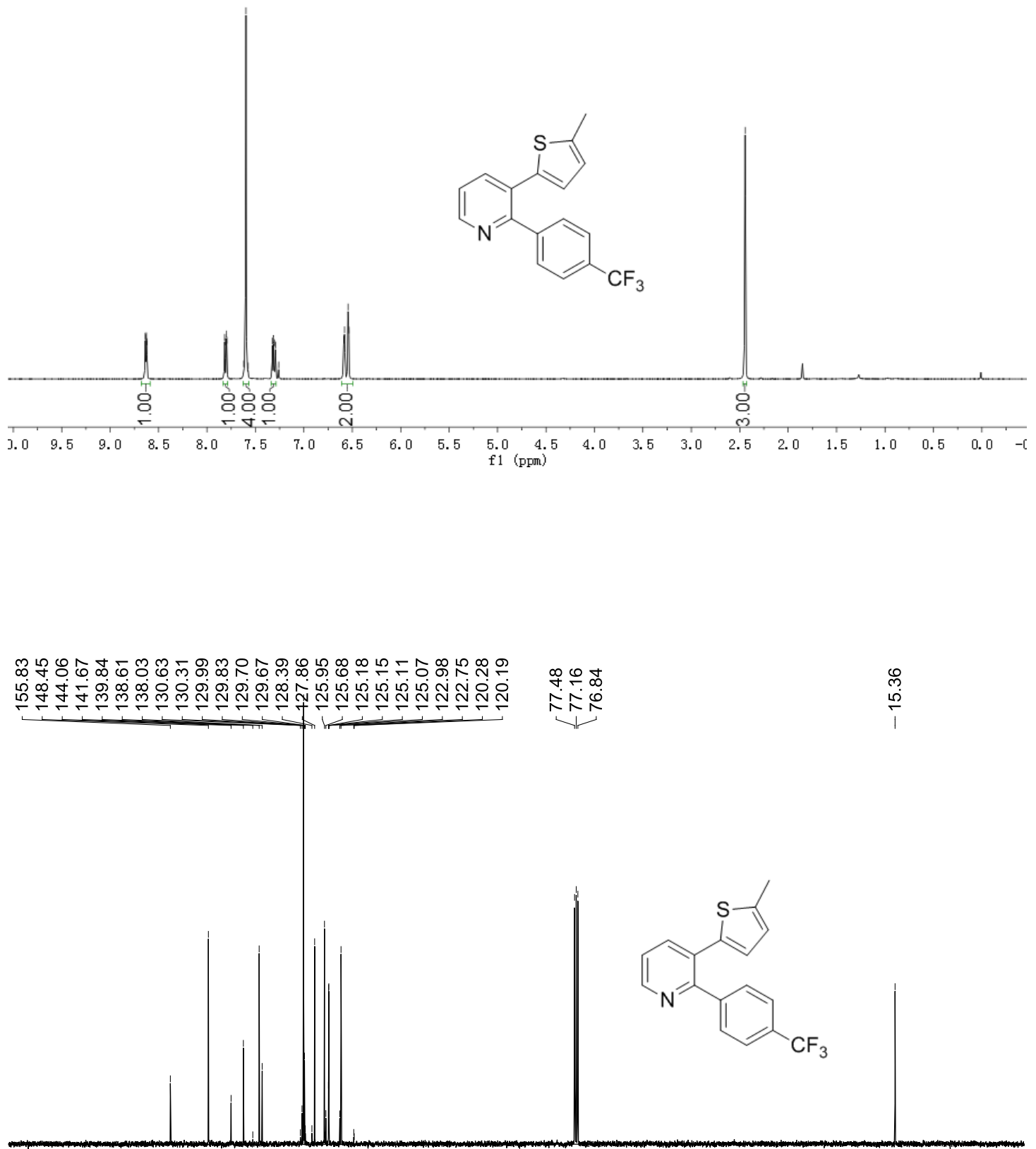

$\begin{array}{lllllllllllllllllll}180 & 170 & 160 & 150 & 140 & 130 & 120 & 110 & 100 & \begin{array}{c}90 \\ \mathrm{f} 1(\mathrm{ppm})\end{array} & 80 & 70 & 60 & 50 & 40 & 30 & 20 & 10 & 0\end{array}$ 


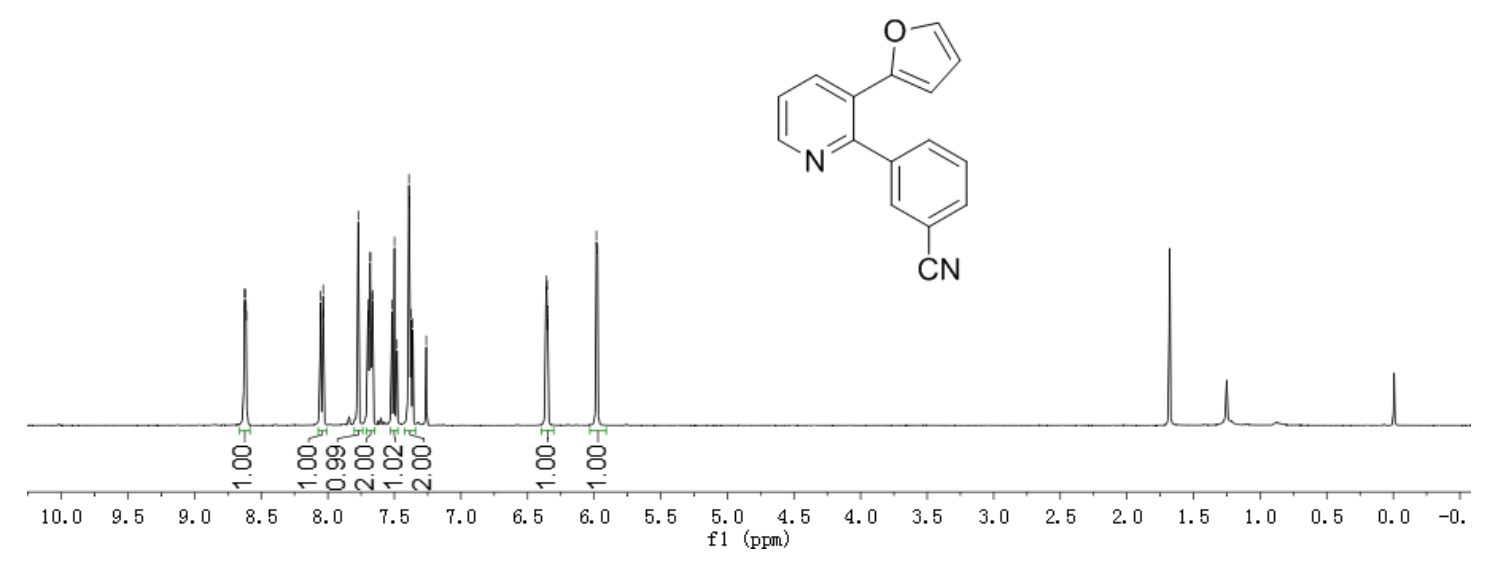

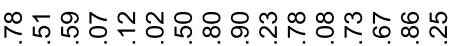

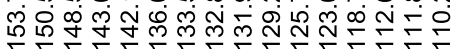
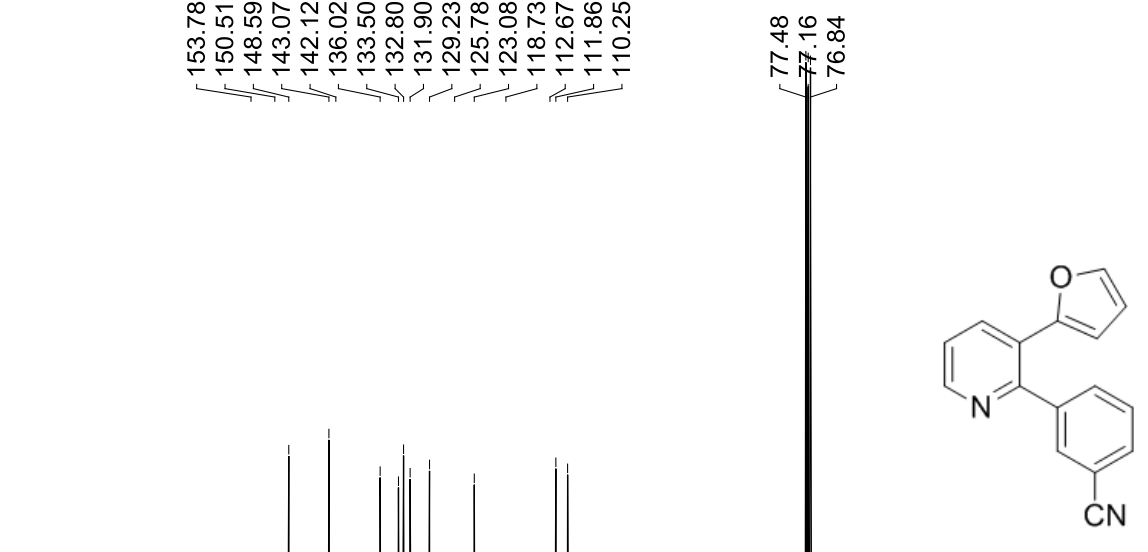

$\begin{array}{llll}180 & 170 & 160 & 150\end{array}$ $140 \quad 130$ $120 \quad 110$ $\mathrm{fl}^{90}(\mathrm{ppm})$ 
(1n)

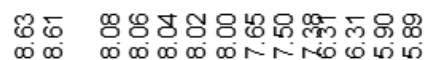

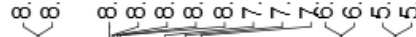

$\stackrel{5}{i}$

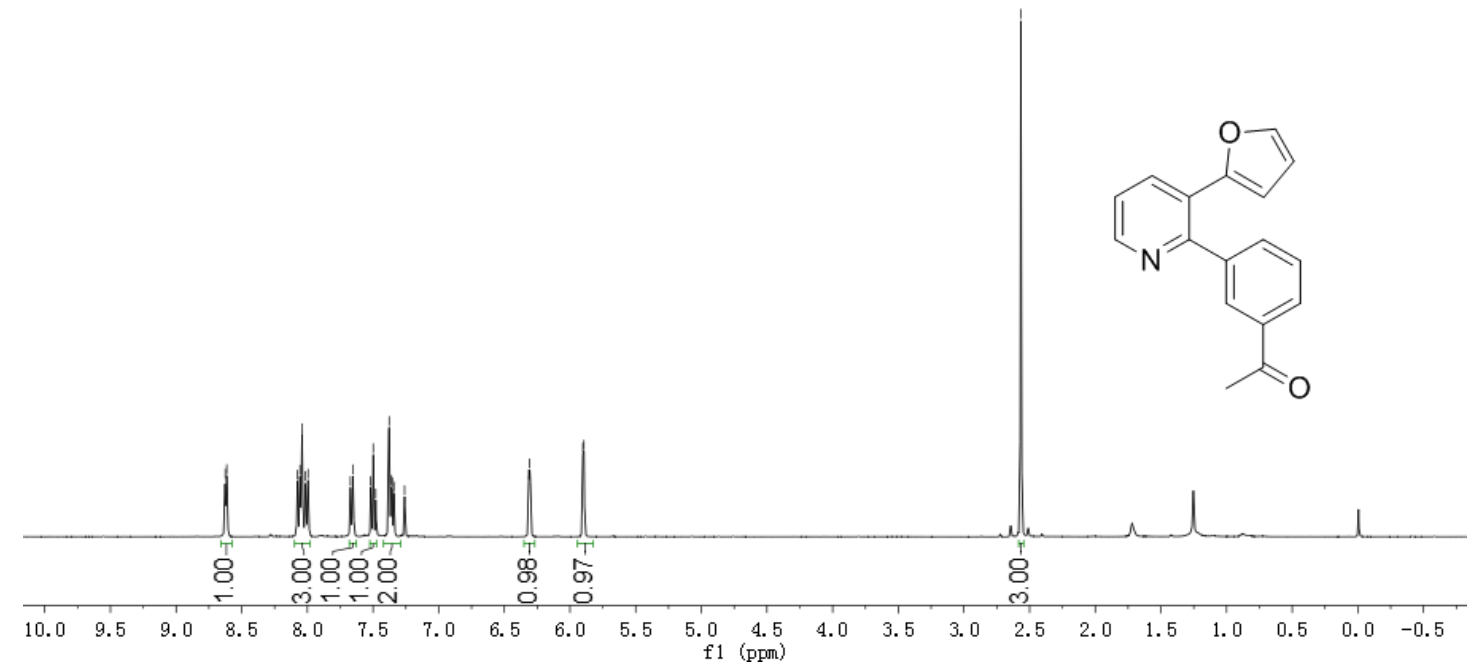

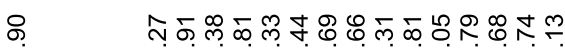

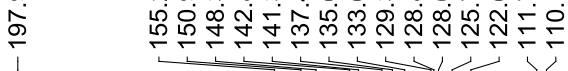

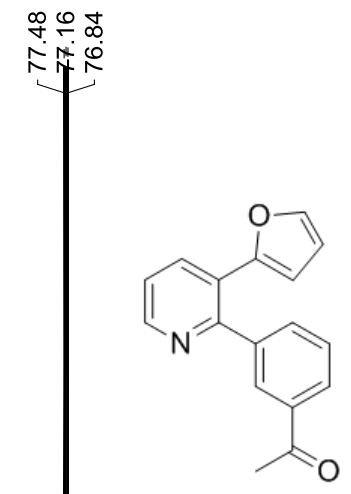

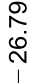

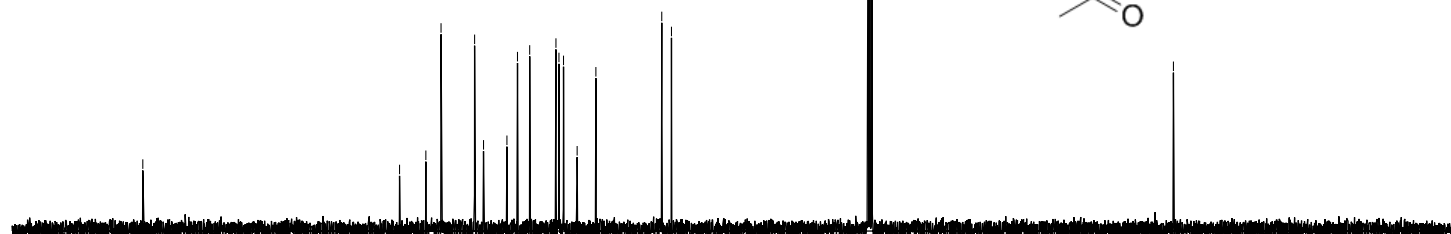

$\begin{array}{lllllllllllllllllllllll}210 & 200 & 190 & 180 & 170 & 160 & 150 & 140 & 130 & 120 & 110 & 100 & 90 & 80 & 70 & 60 & 50 & 40 & 30 & 20 & 10 & 0 & -10\end{array}$

(10) 


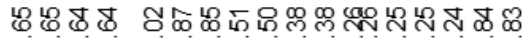

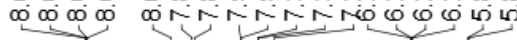

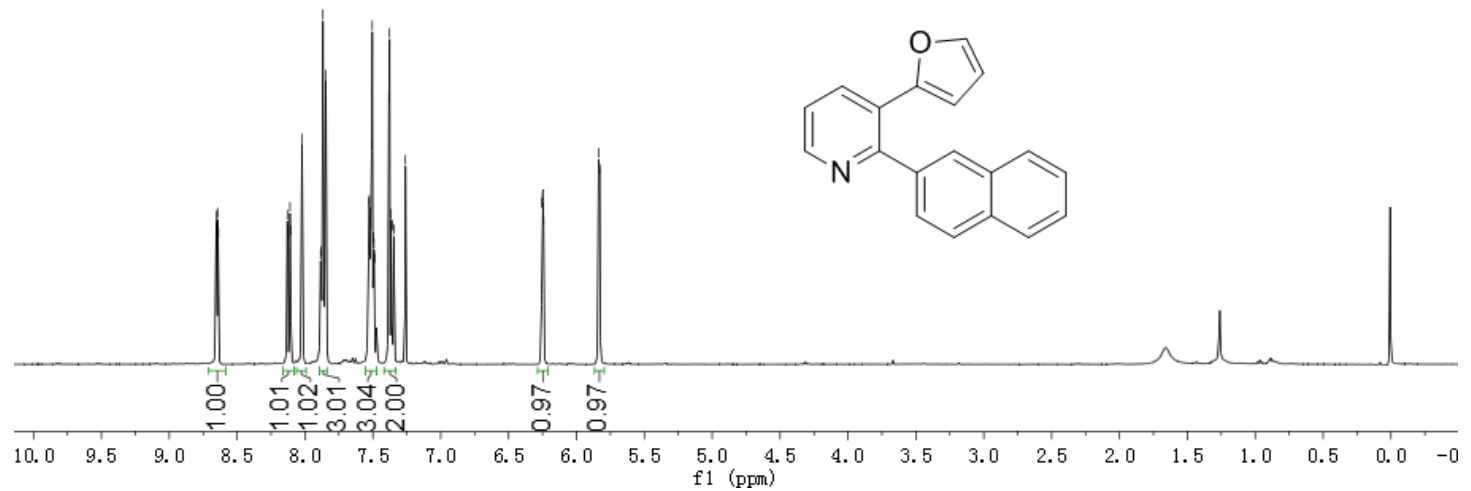

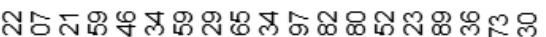

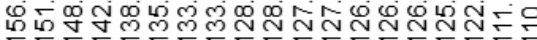
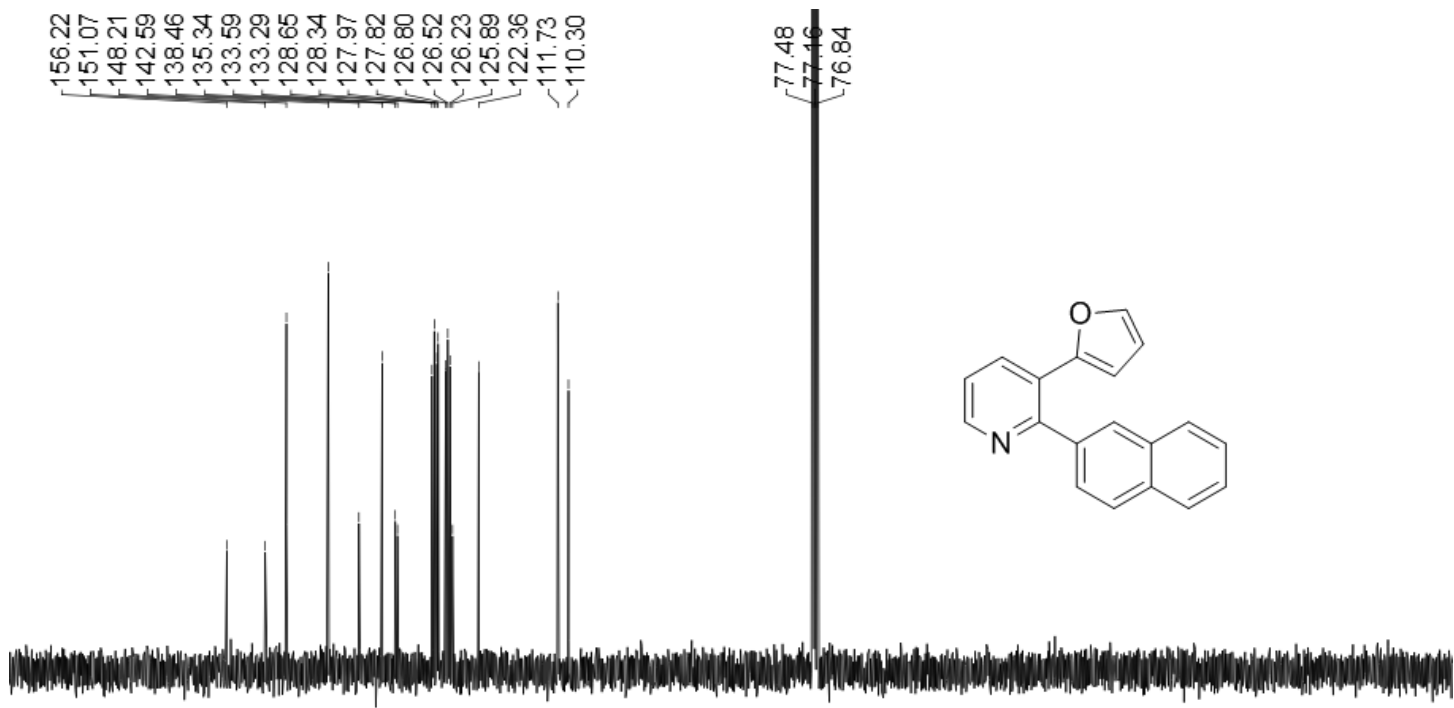

$180 \quad 1$

150

$130 \quad 120$

$10090 \quad 80$

7060

40

$20 \quad 10$ 


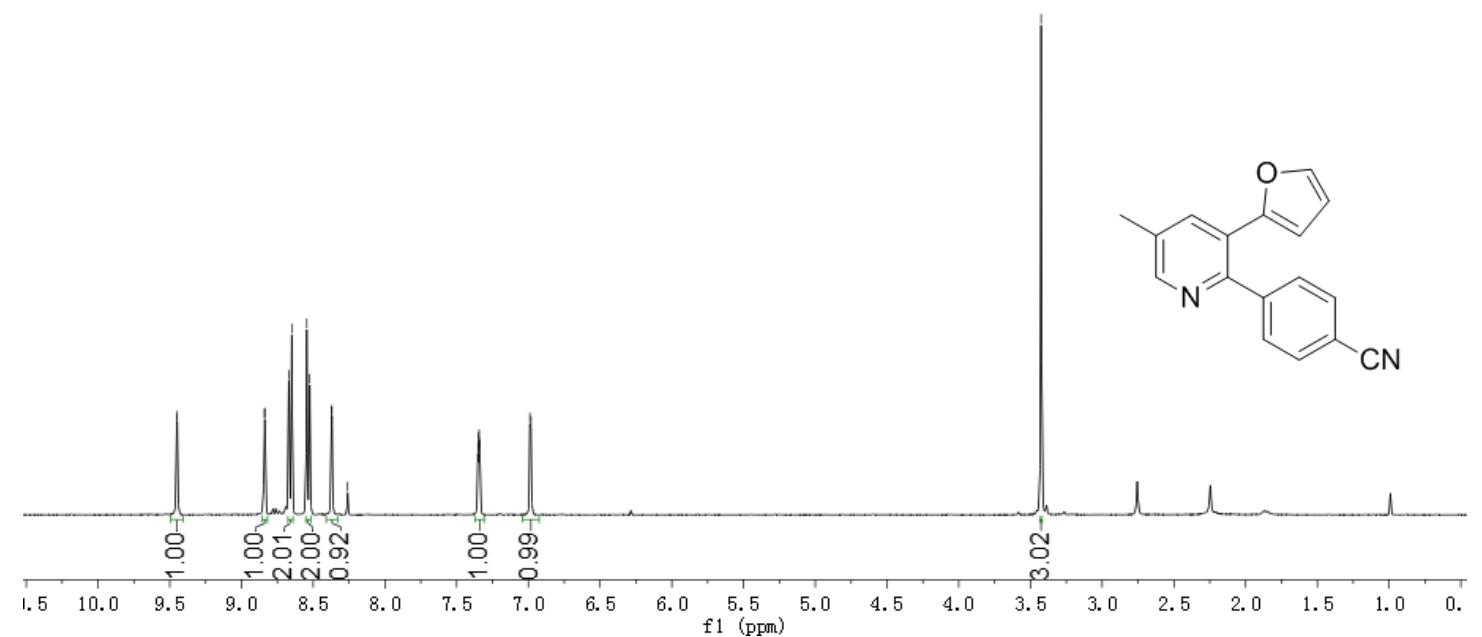

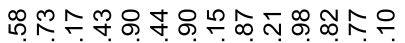

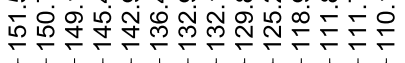

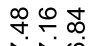
रำ

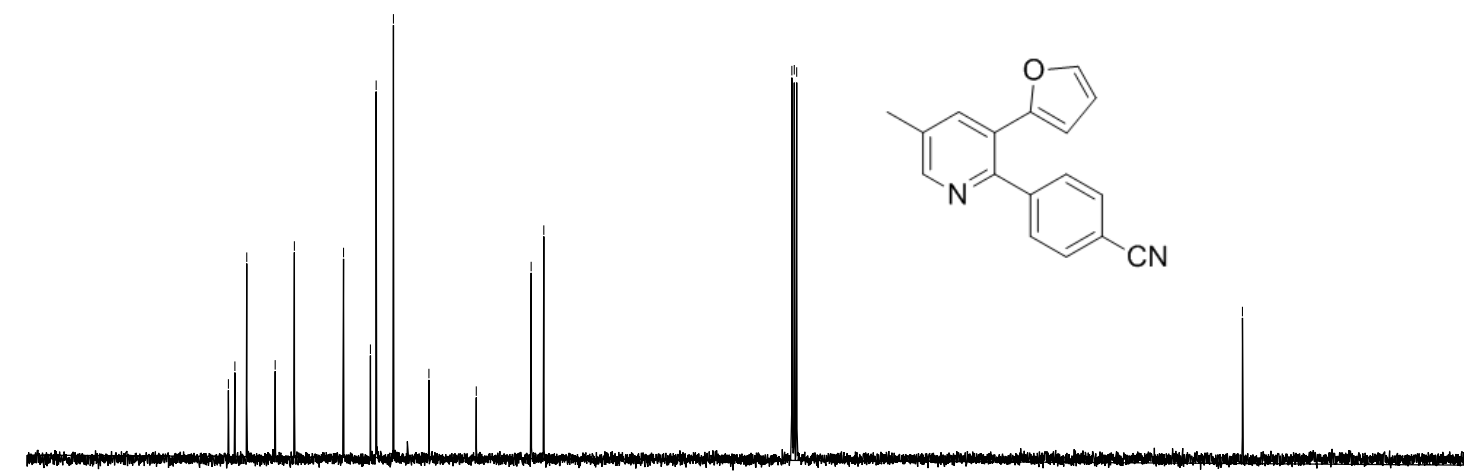




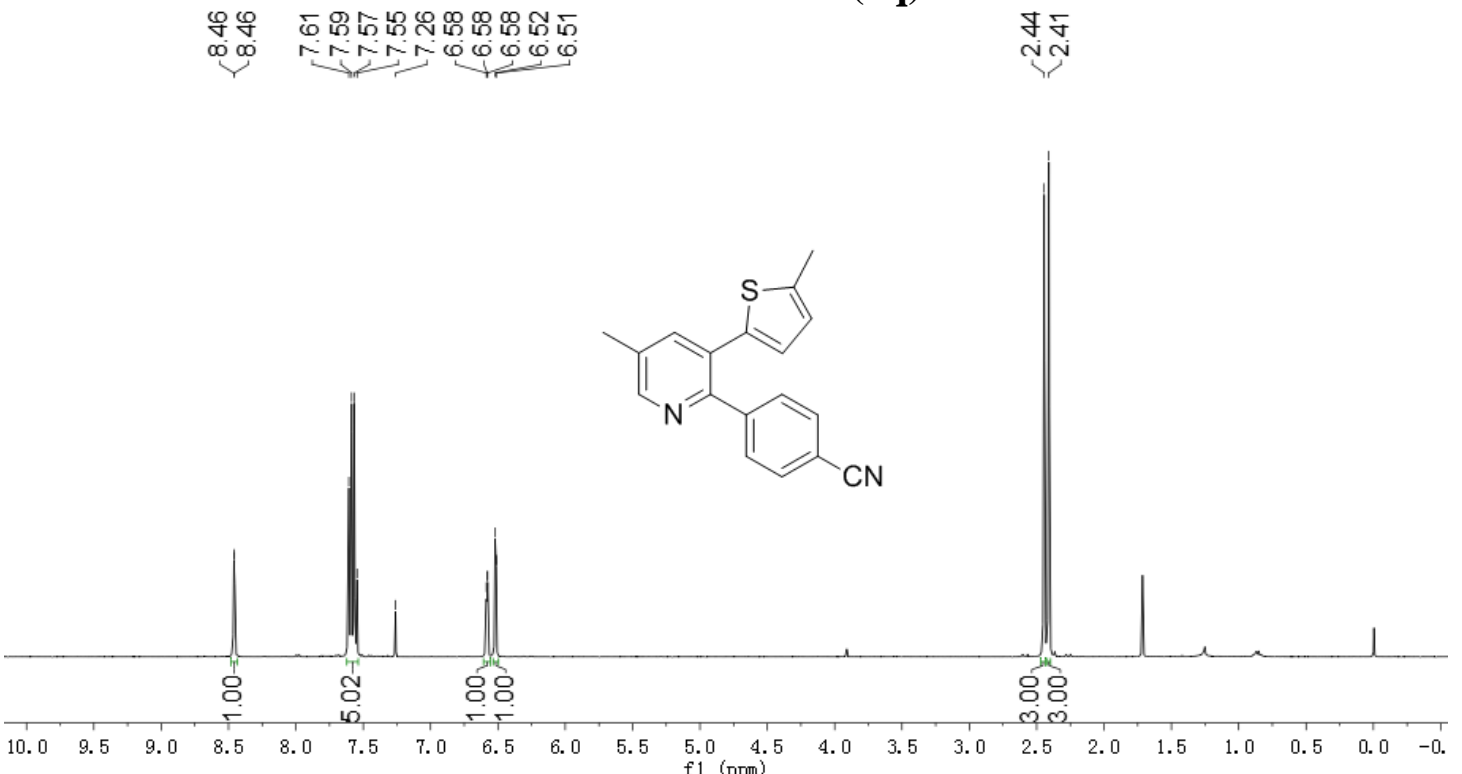

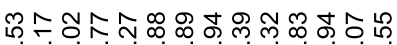

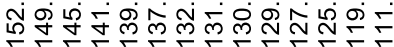

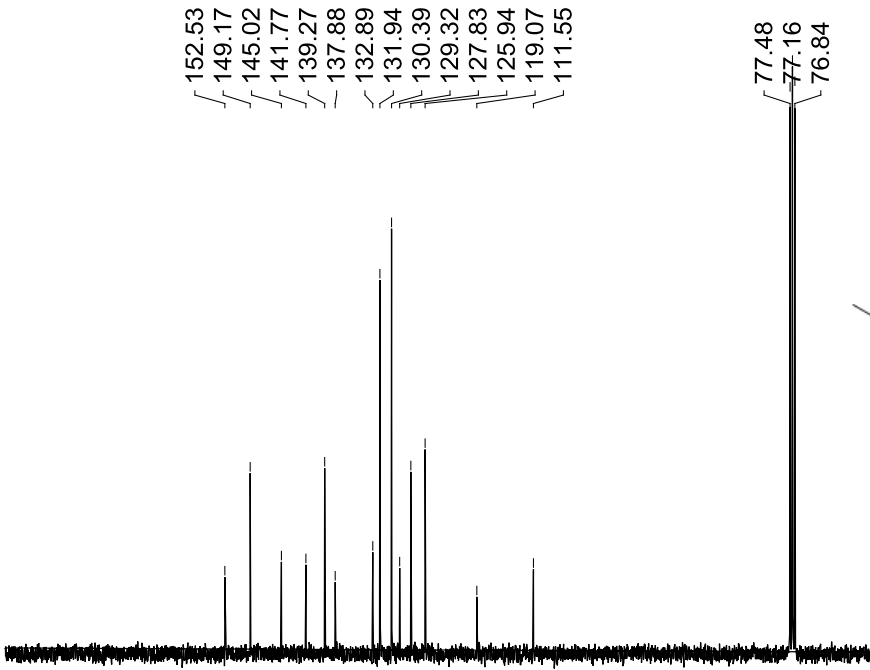

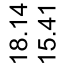

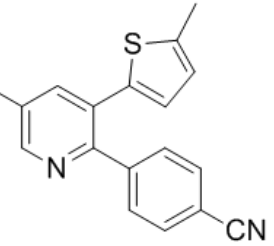

$\begin{array}{llllllllll}180 & 170 & 160 & 150 & 140 & 130 & 120 & 110 & 100 & 90\end{array}$ 


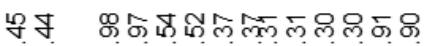

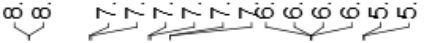

(1r)

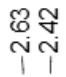
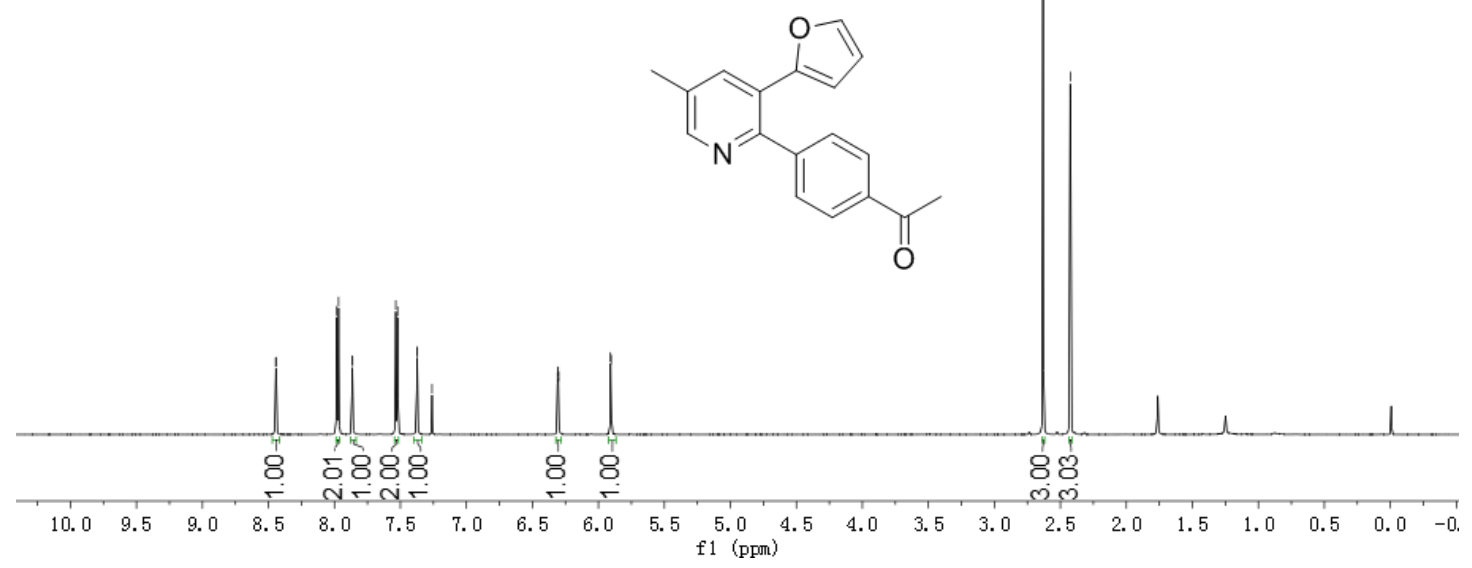

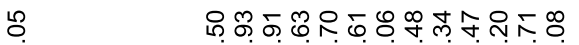

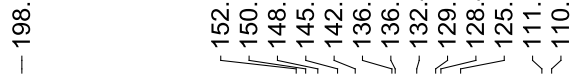

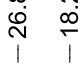

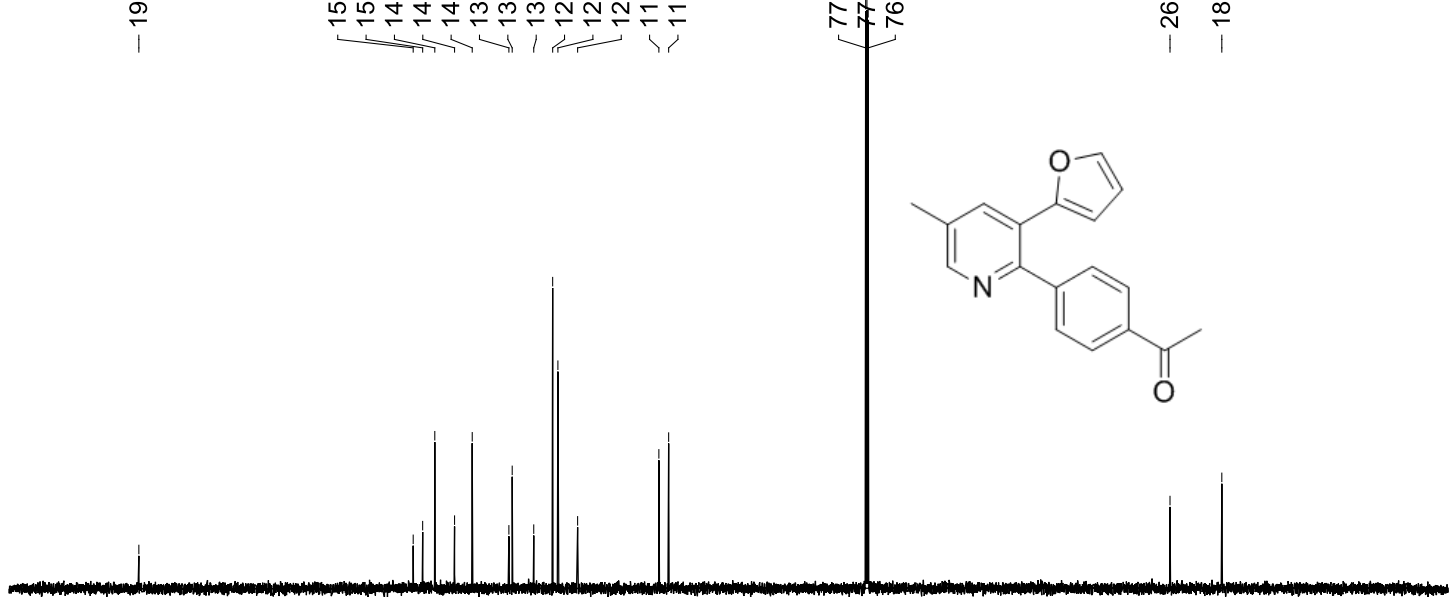

$\begin{array}{lllllllllllllllllllllll}210 & 200 & 190 & 180 & 170 & 160 & 150 & 140 & 130 & 120 & 110 & 100 & 90 & 80 & 70 & 60 & 50 & 40 & 30 & 20 & 10 & 0 & -10\end{array}$ 
(1s)

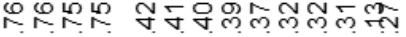

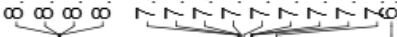

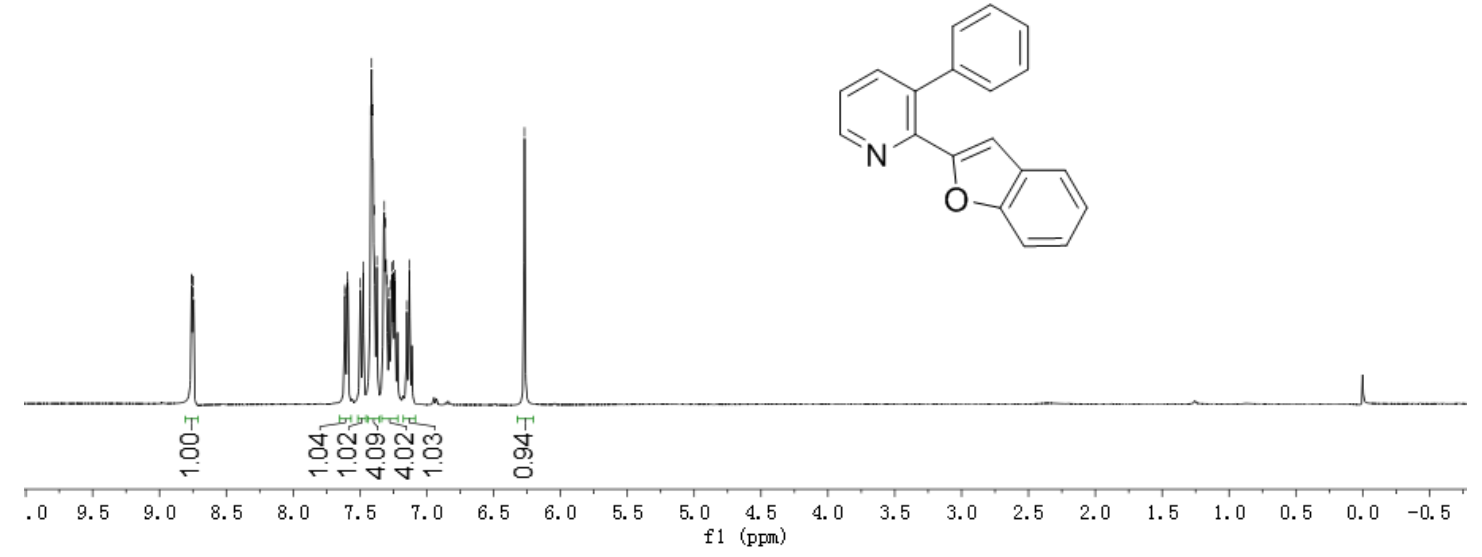

מํ.

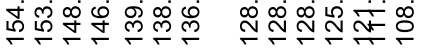

$\stackrel{\infty}{\sim} \div$

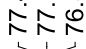

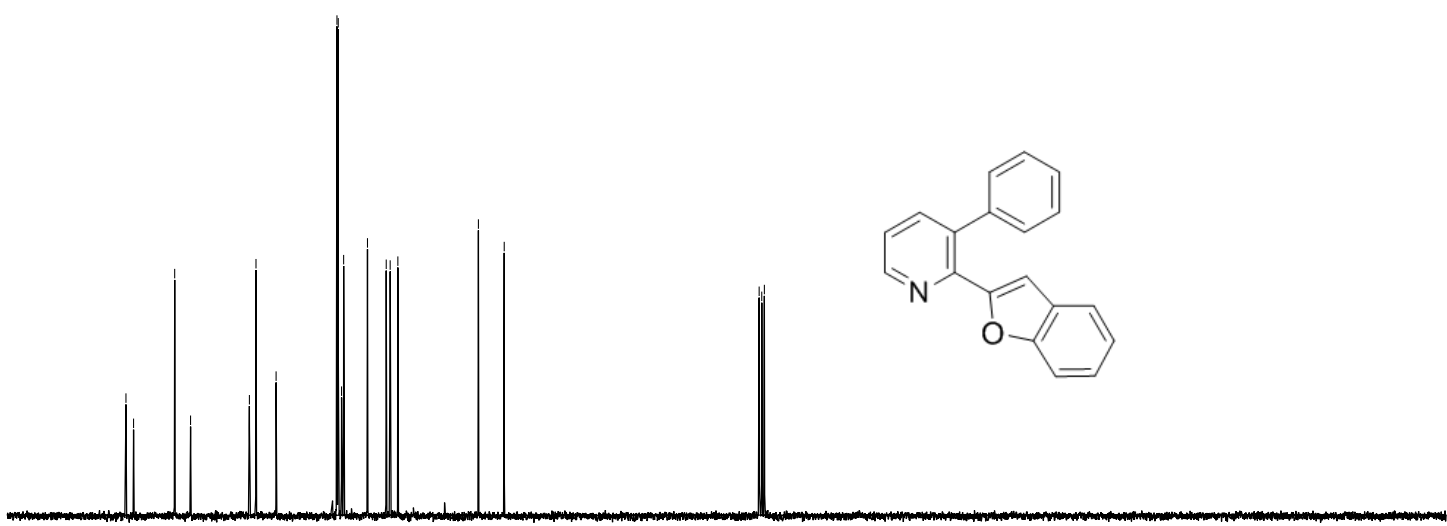

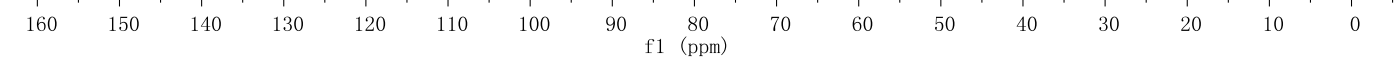


(1t)

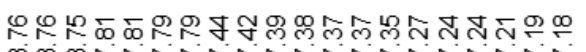

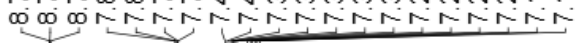

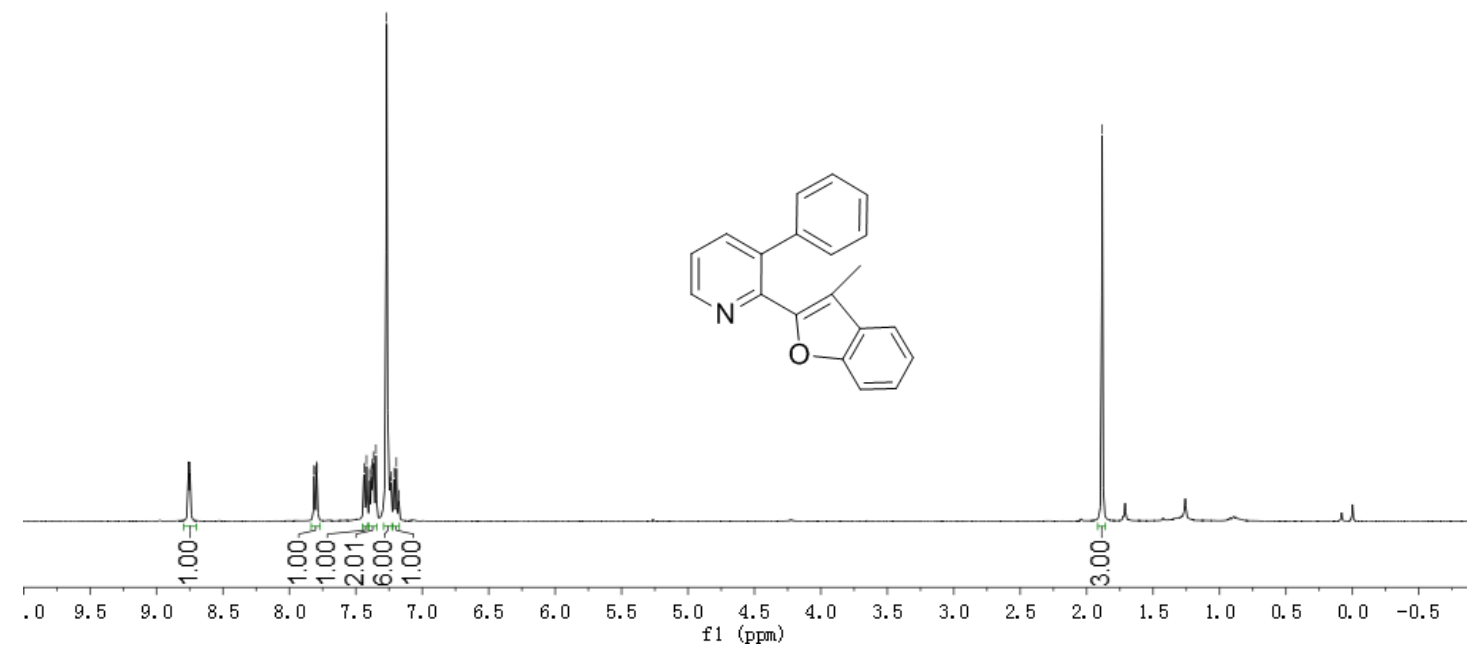

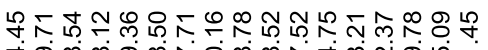

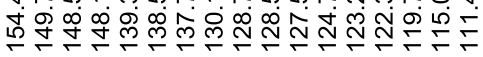

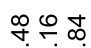

송

0
$\infty$
$\infty$
1

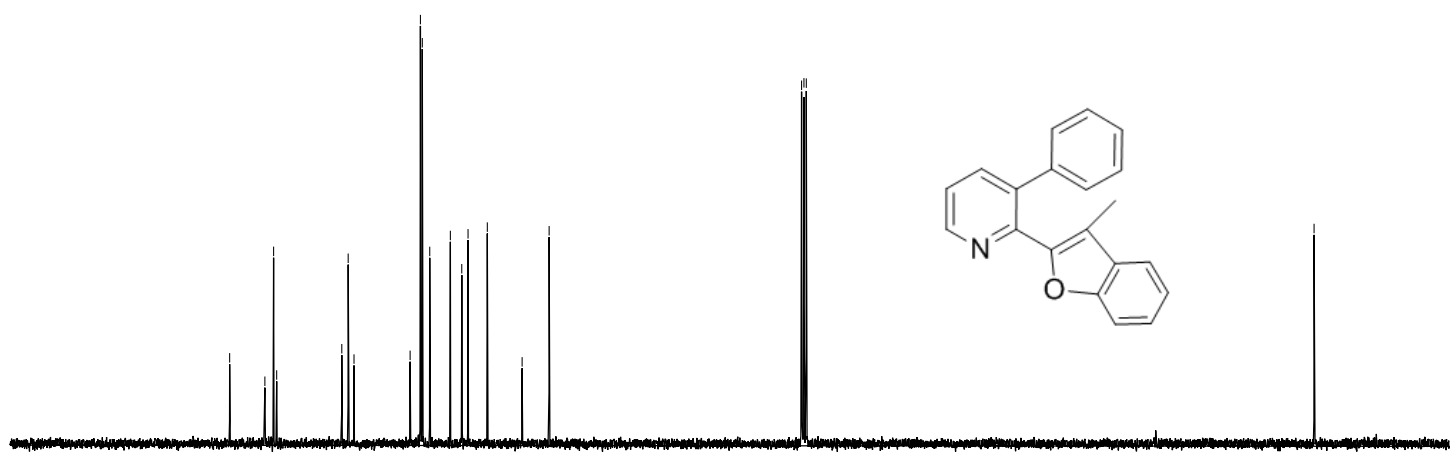

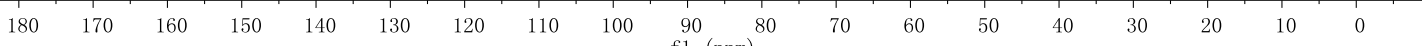


(3a)

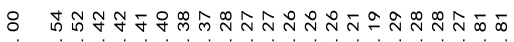

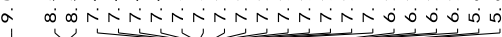

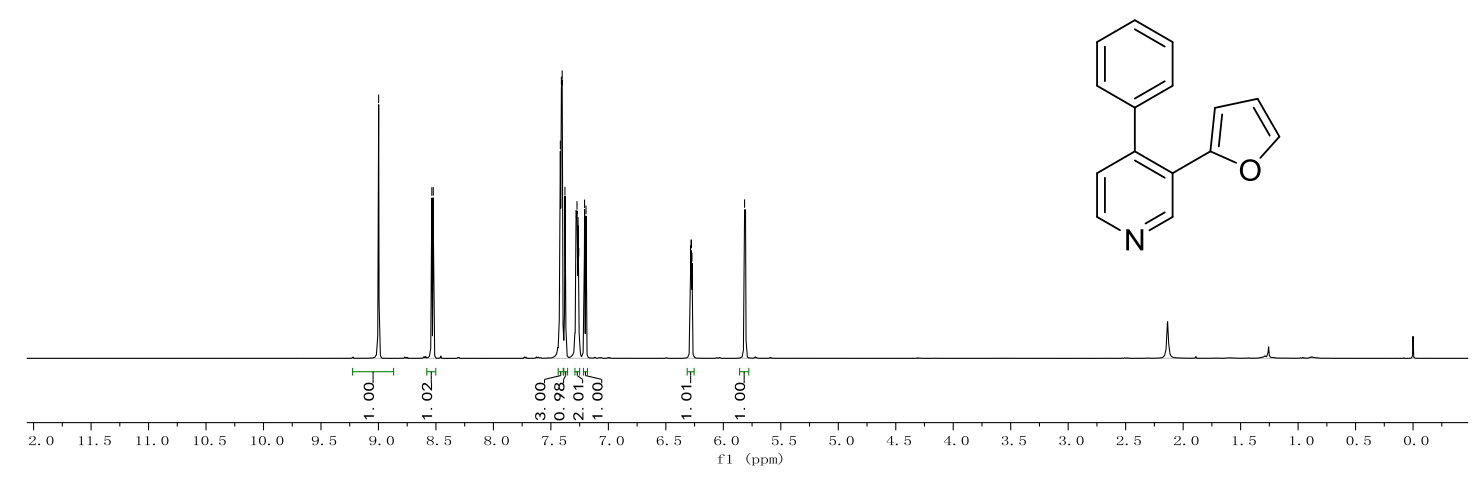

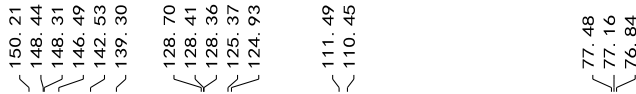

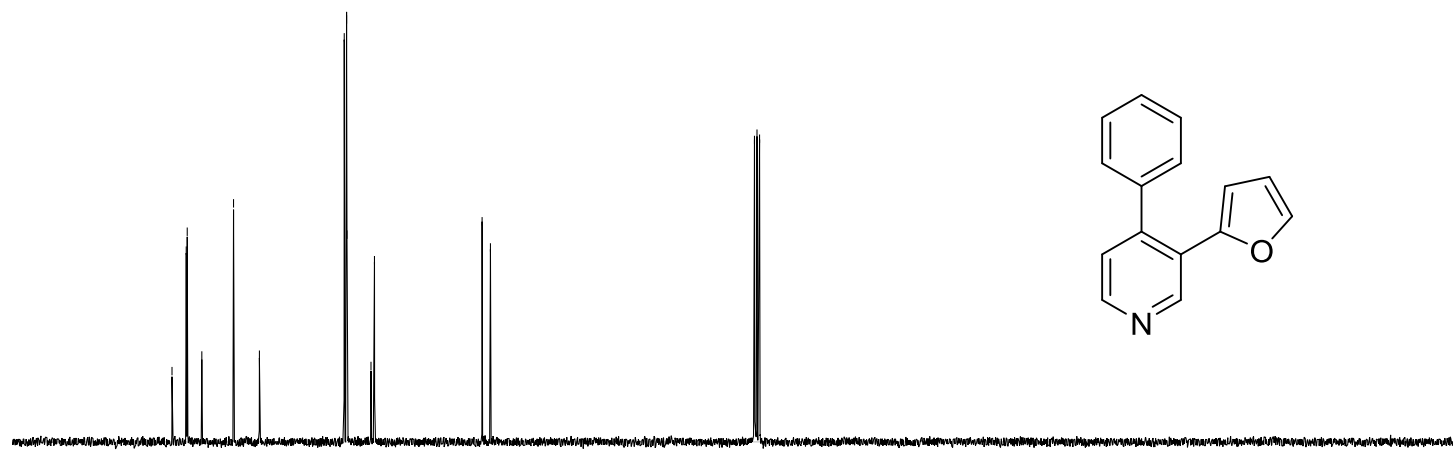

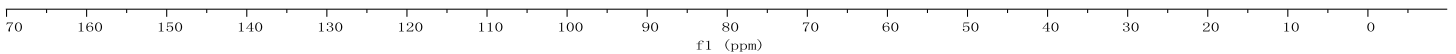


(3b)

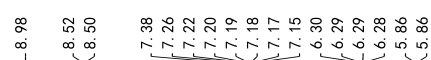

$\underset{\substack{1 \\ i}}{1}$

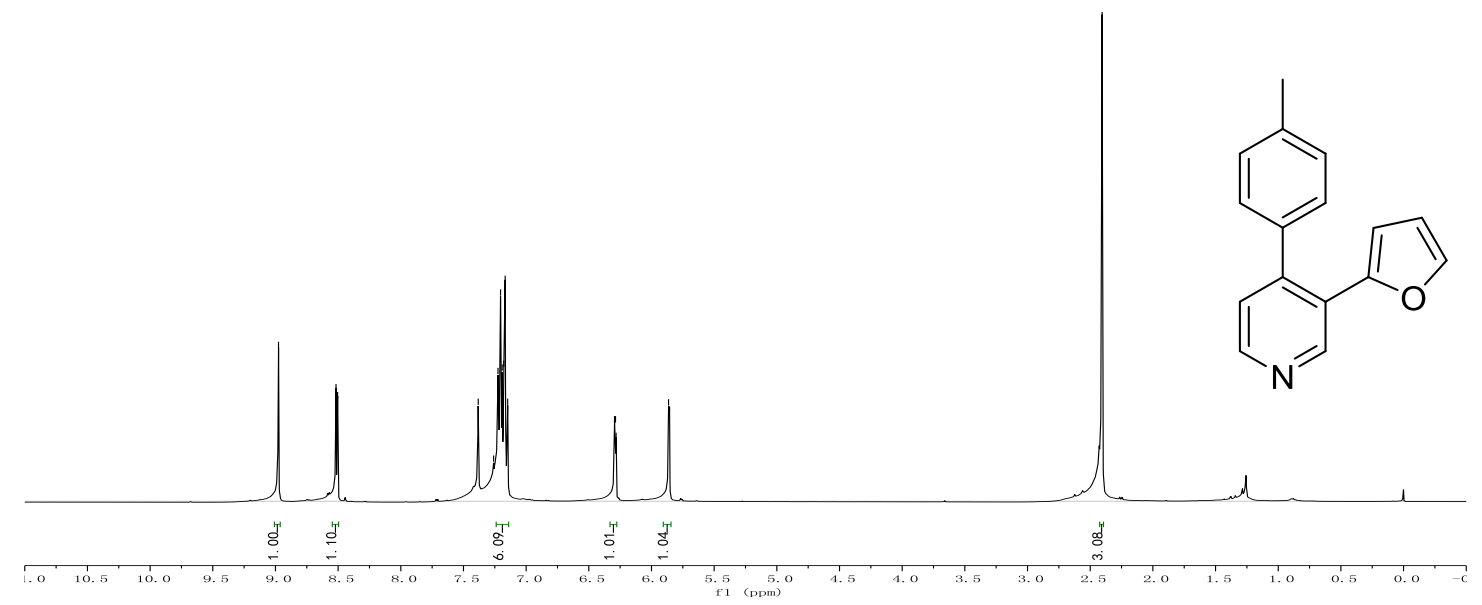

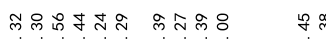

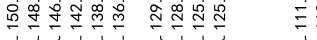

乐ํํㅇ

Кำ

$\stackrel{\infty}{m}$

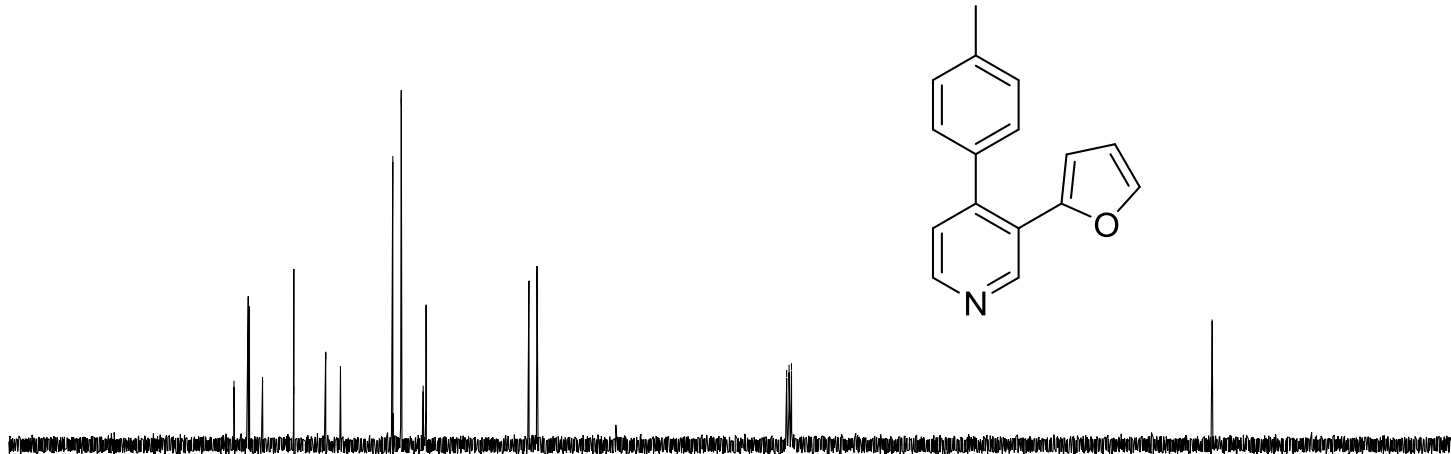


(3c)

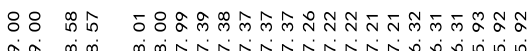

$\sigma a v^{\infty} v^{\infty}$
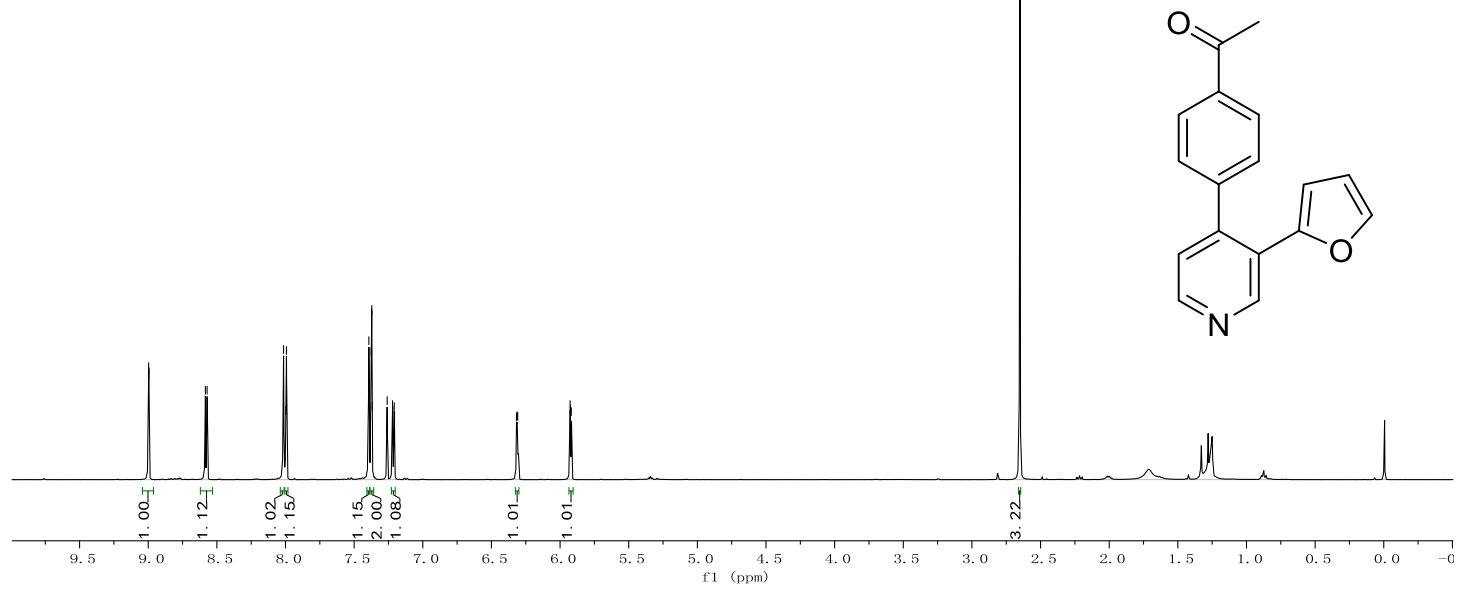

$\frac{n}{2}$

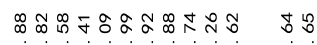

$\underbrace{\infty}$

$\stackrel{m}{\infty}$

福

กั

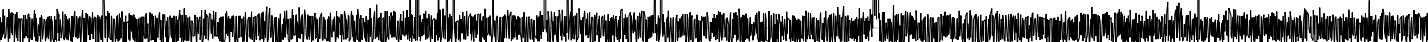

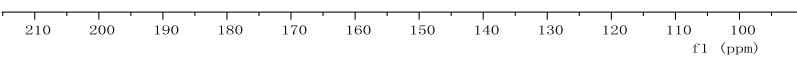


(3d)

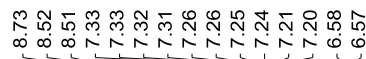

$\stackrel{\infty}{\sim}$

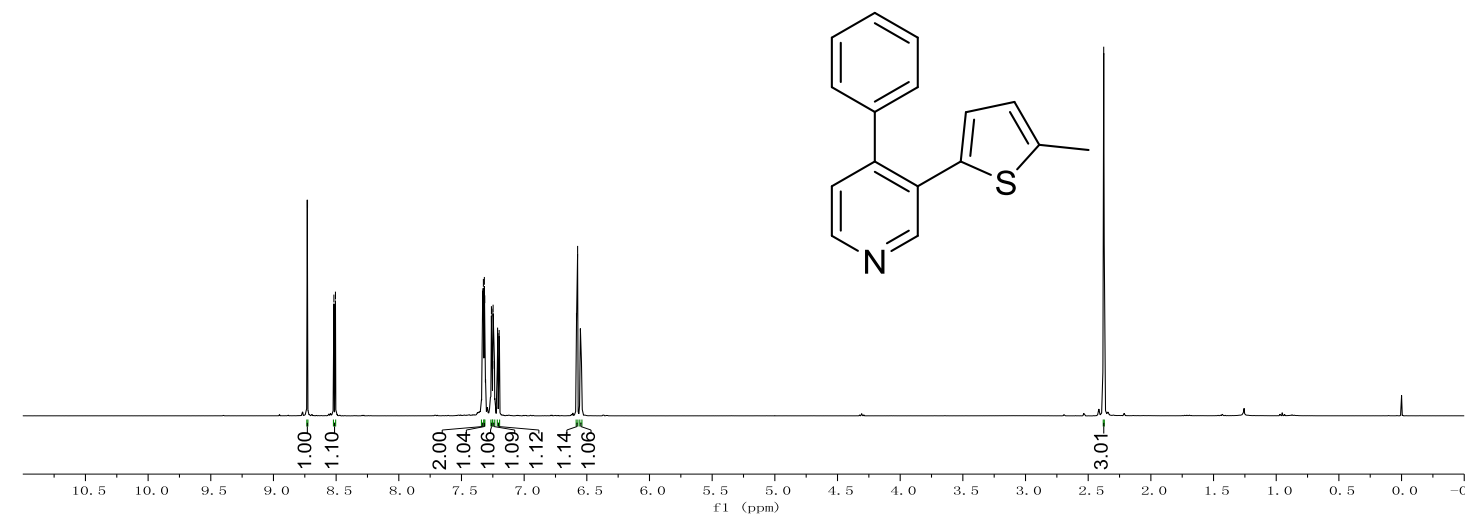

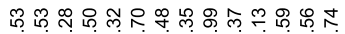

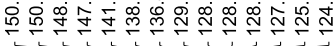

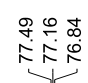

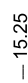

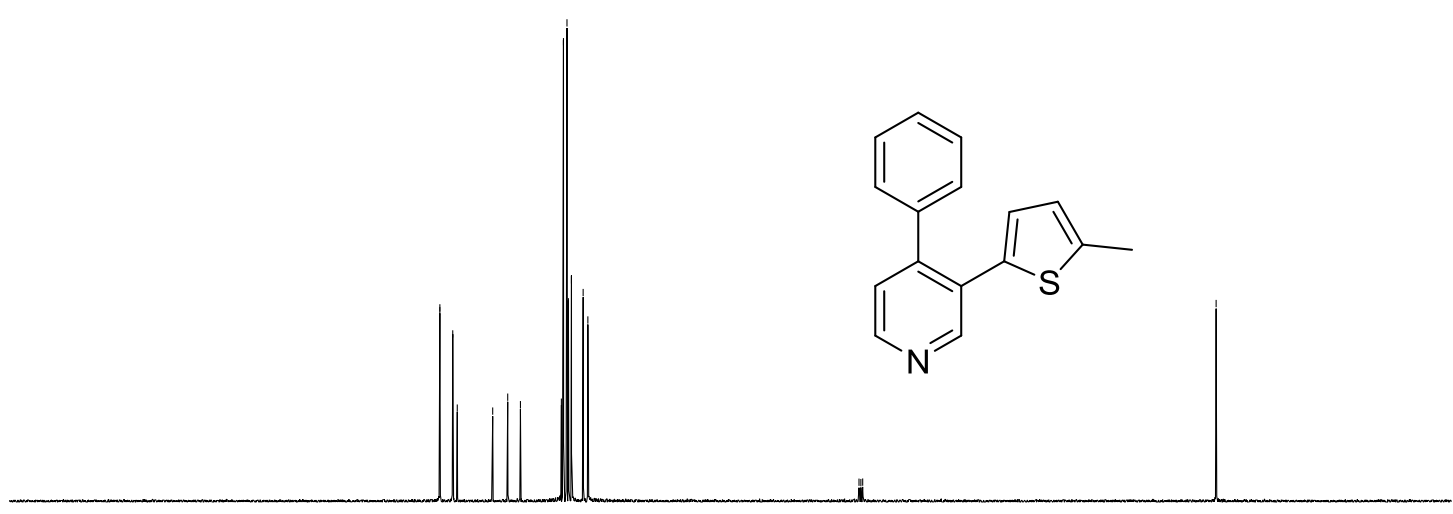

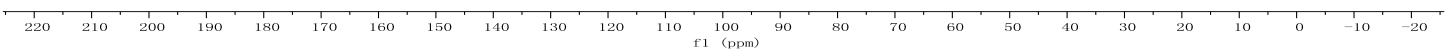


(4a)

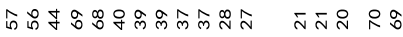

o,

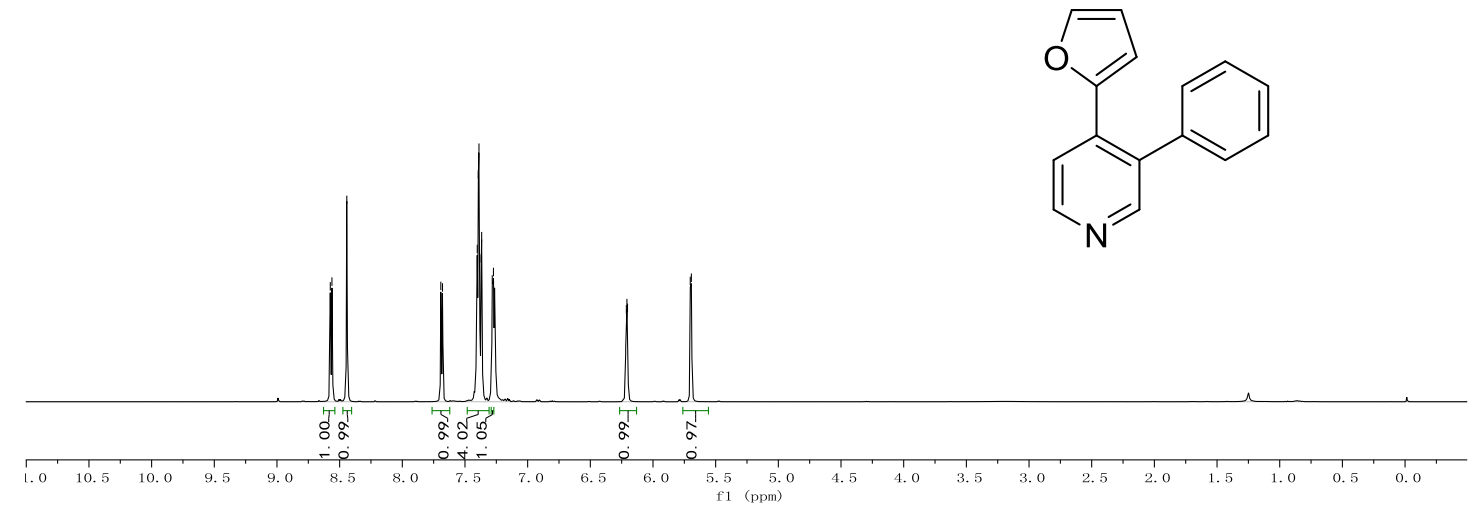

ธ요

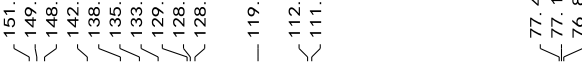

舟요

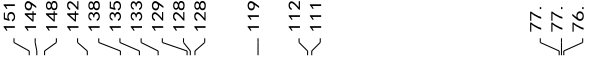
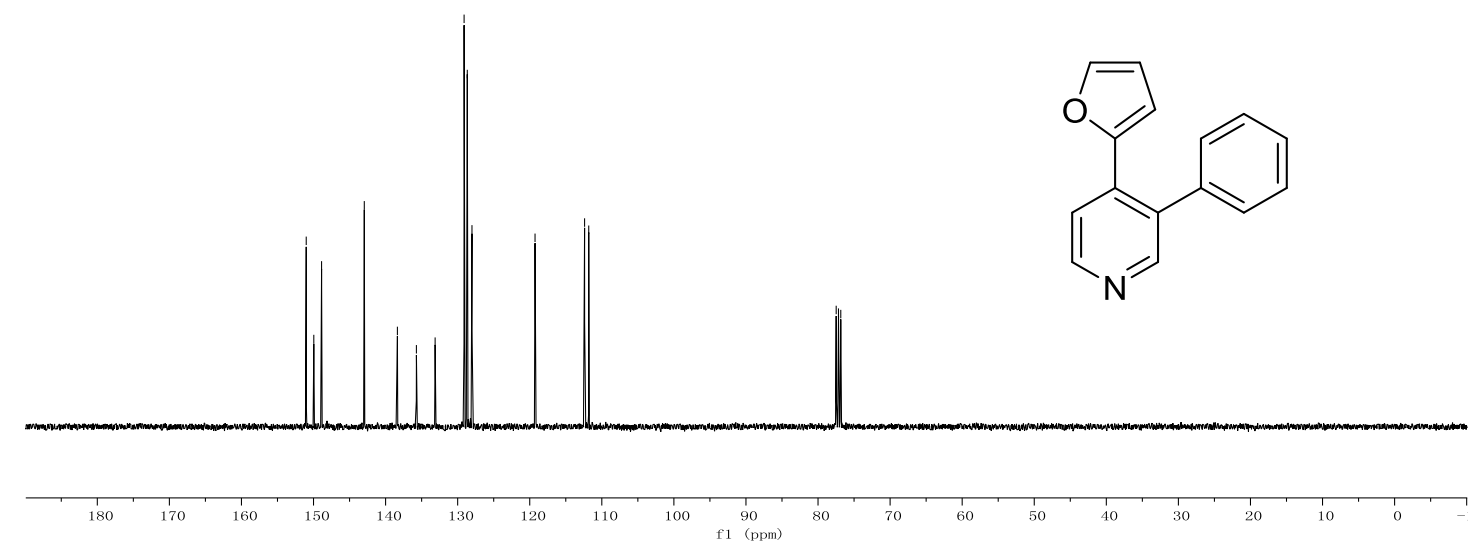
(4b)

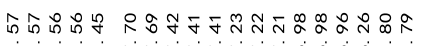

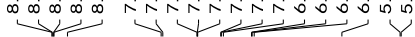

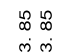

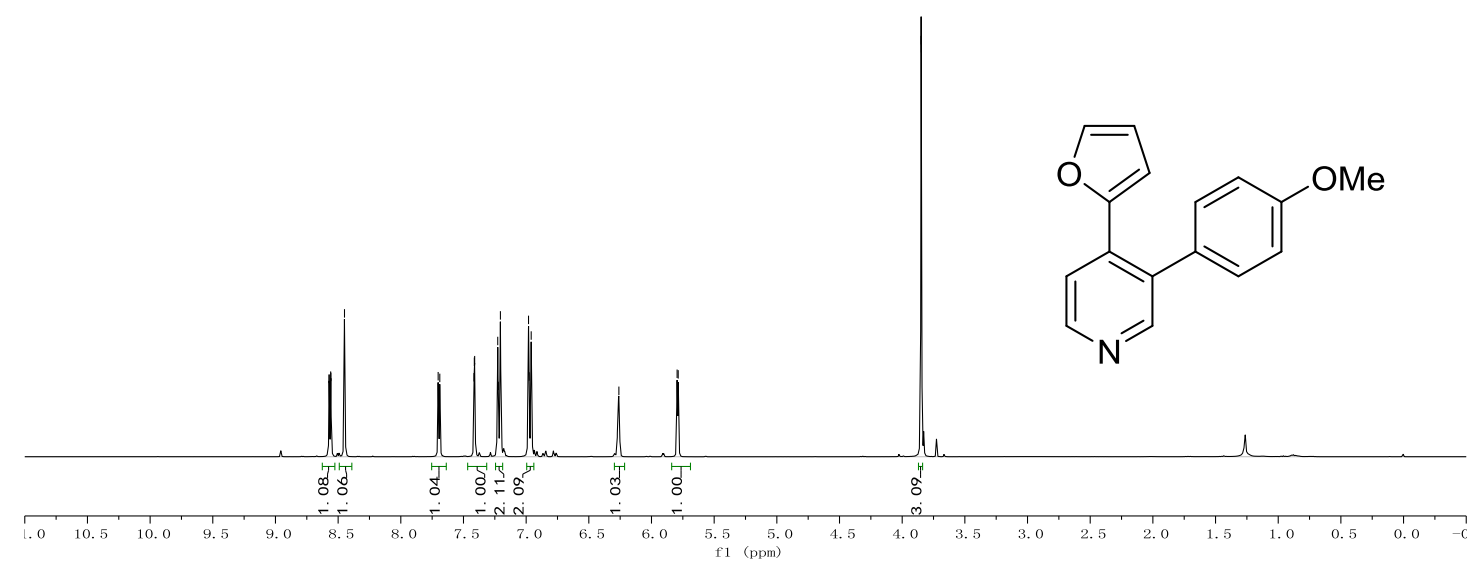

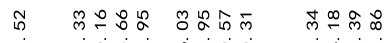

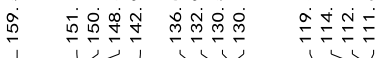

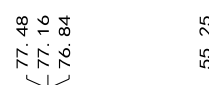

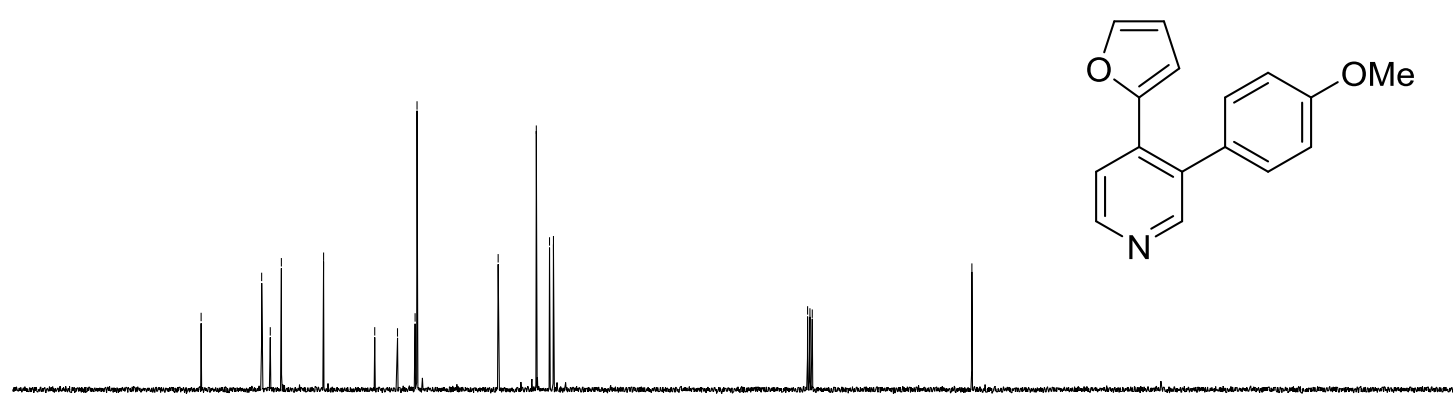


(4b)

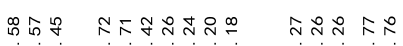

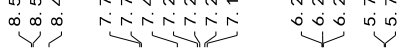

$\stackrel{\substack{\text { i } \\ \text { ! }}}{2}$

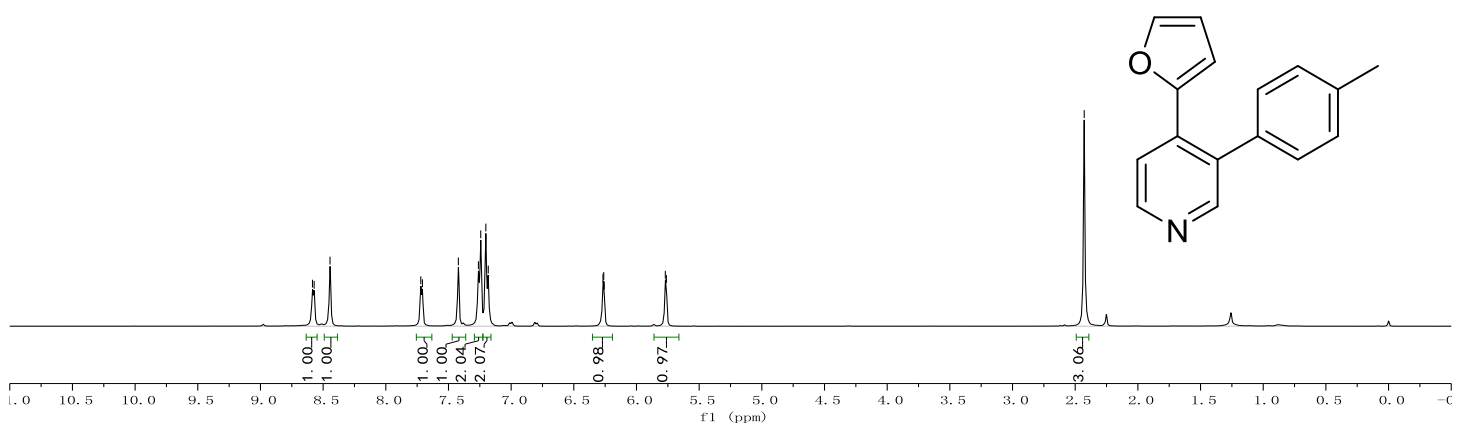

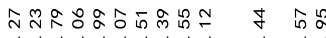

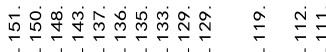

$\stackrel{\infty}{\sim} \stackrel{\infty}{\infty}$

กิ゚

우

जा

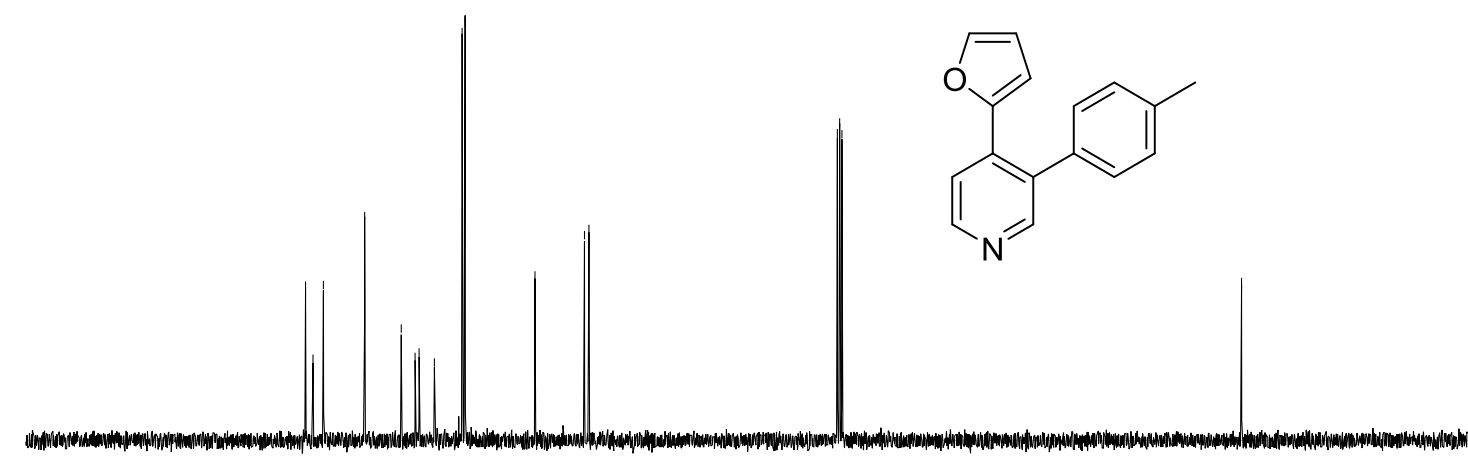

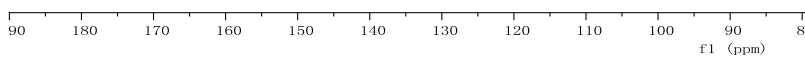




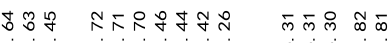

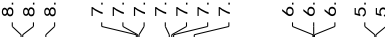
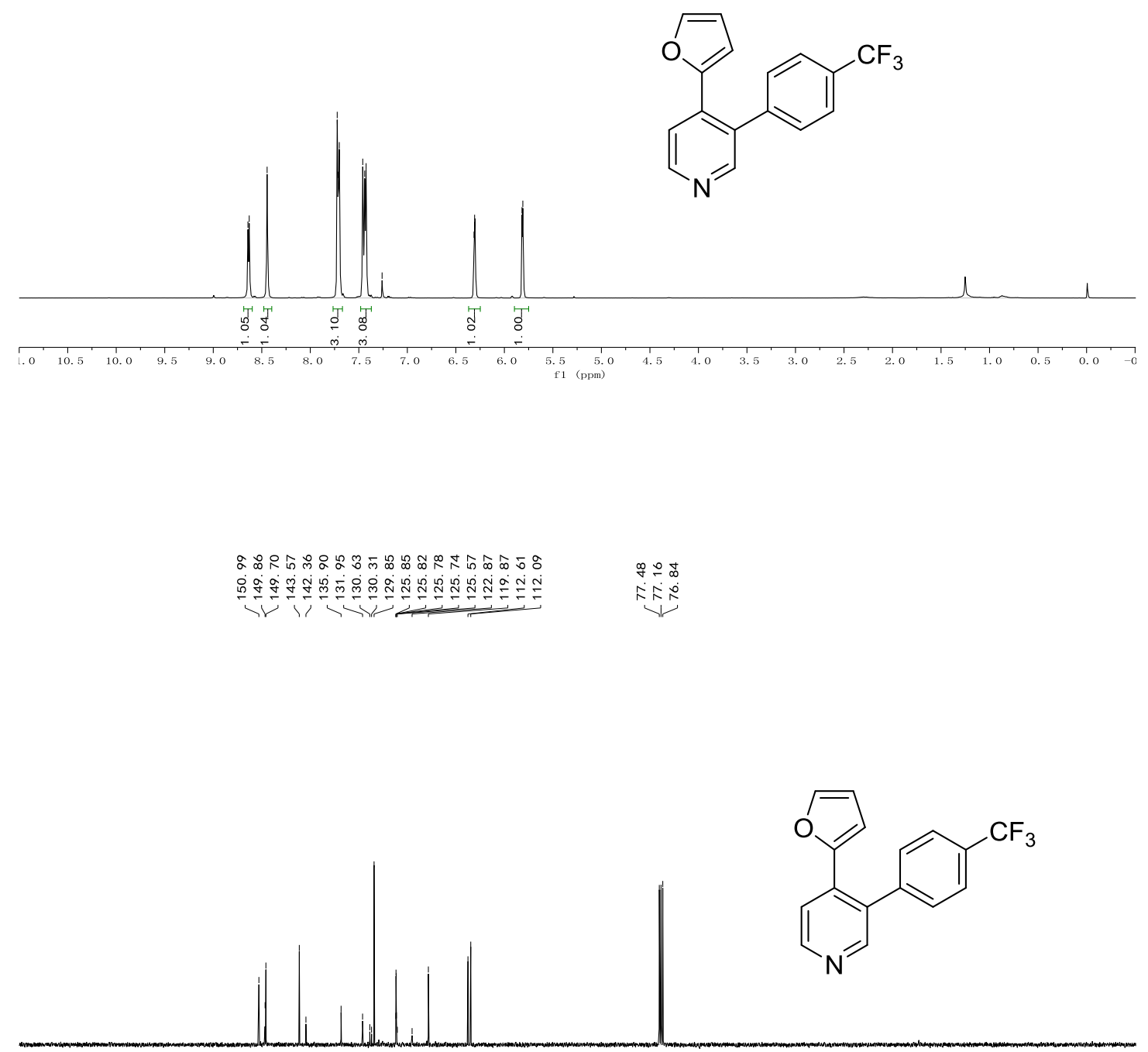
(4e)
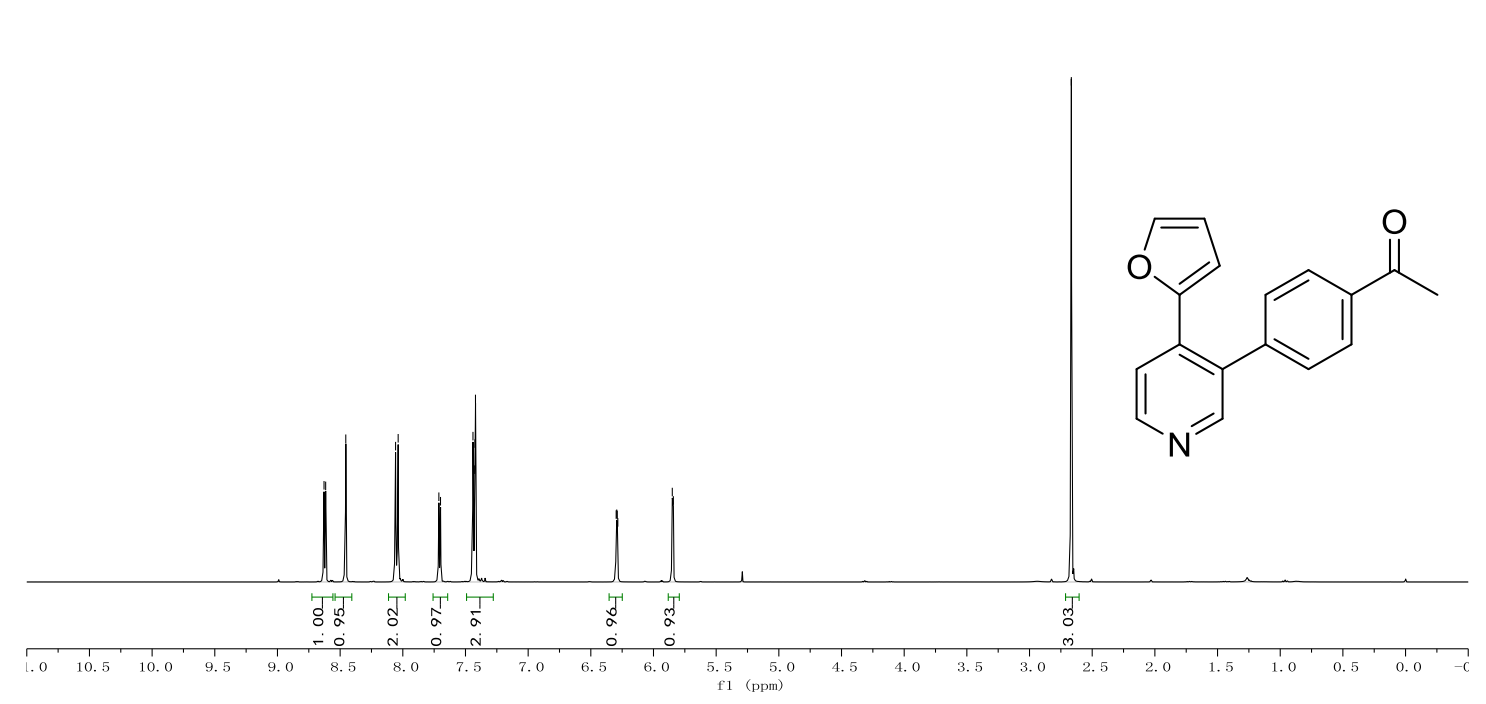

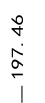

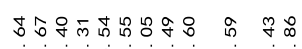

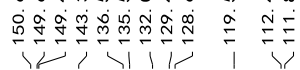

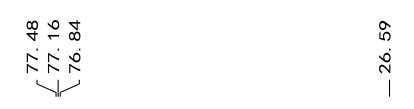

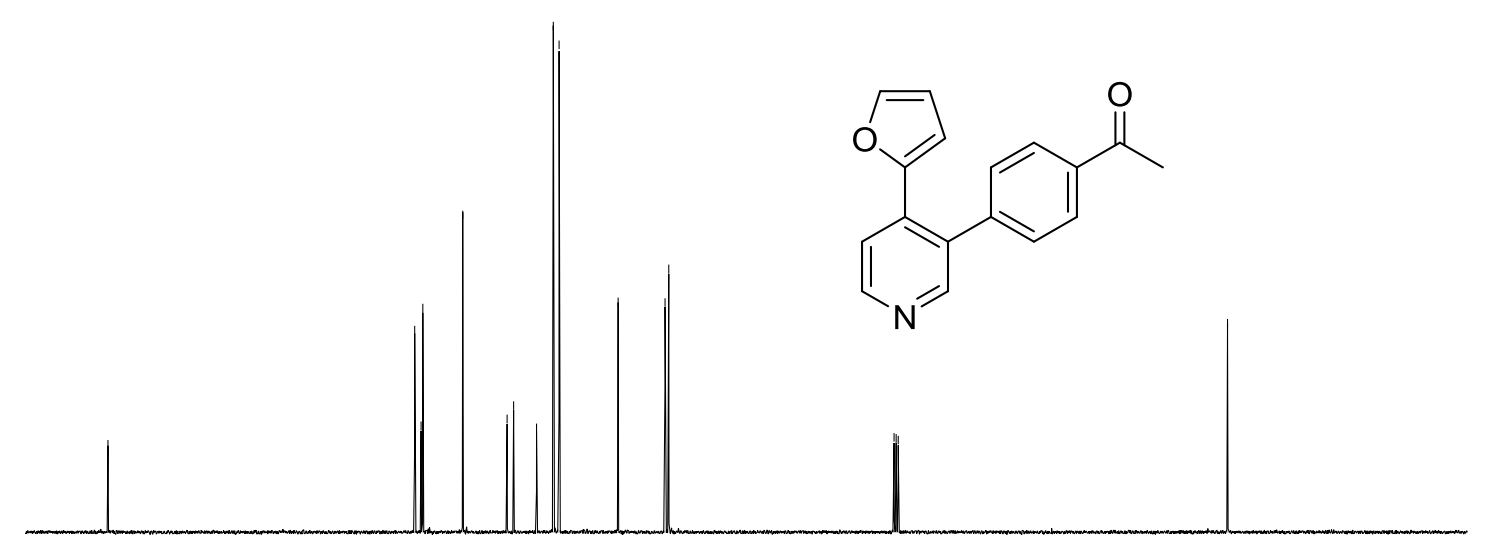

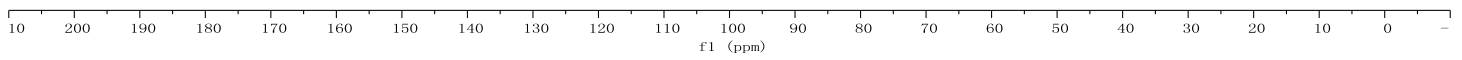


(4f)

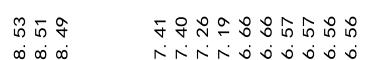

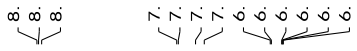

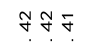

$\int_{i n d}^{4 i n}$

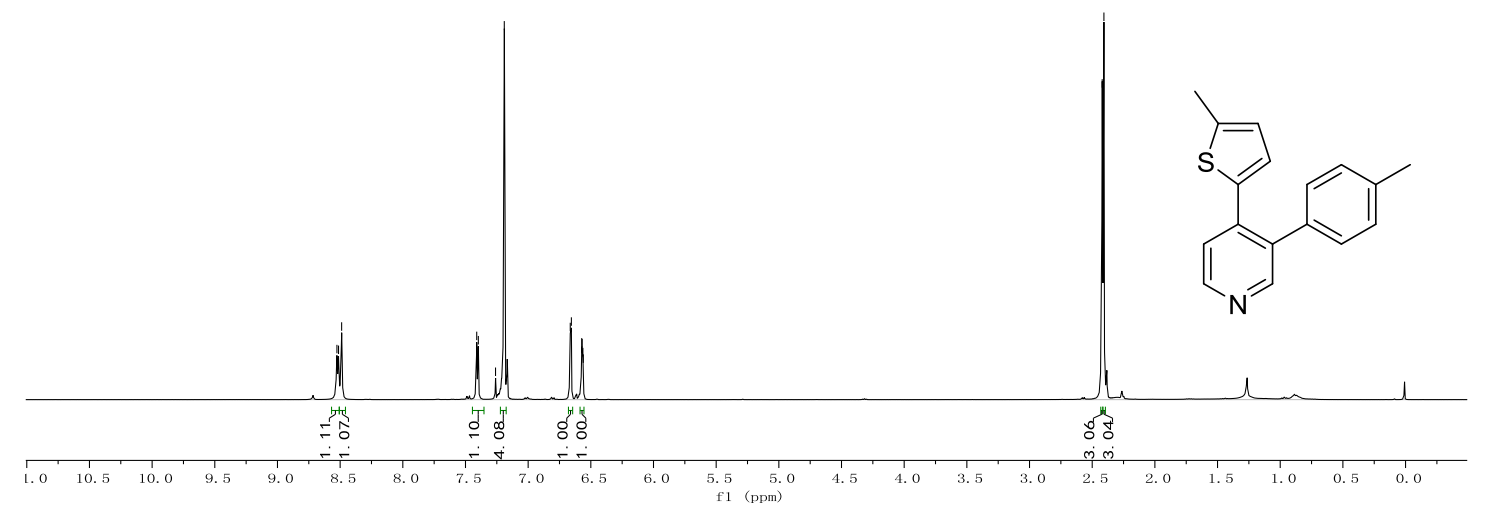

旨ㅇㅇㅇํำ

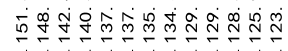

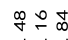

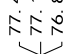

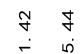

1)

$\stackrel{1}{i}$

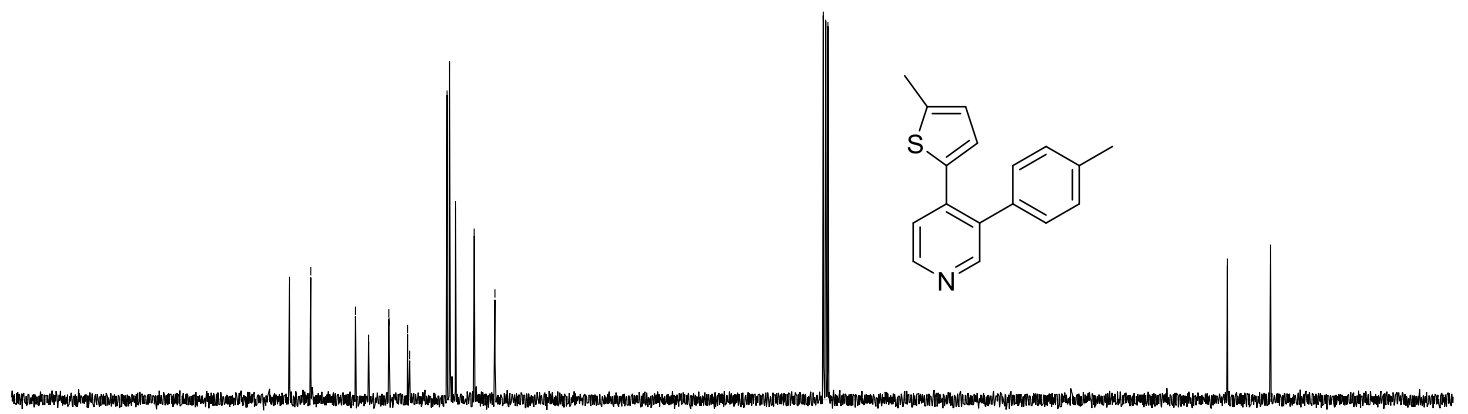

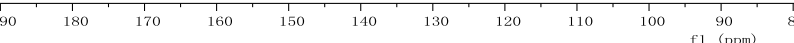


(4g)

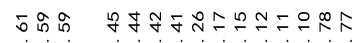

$e_{\infty}$

2

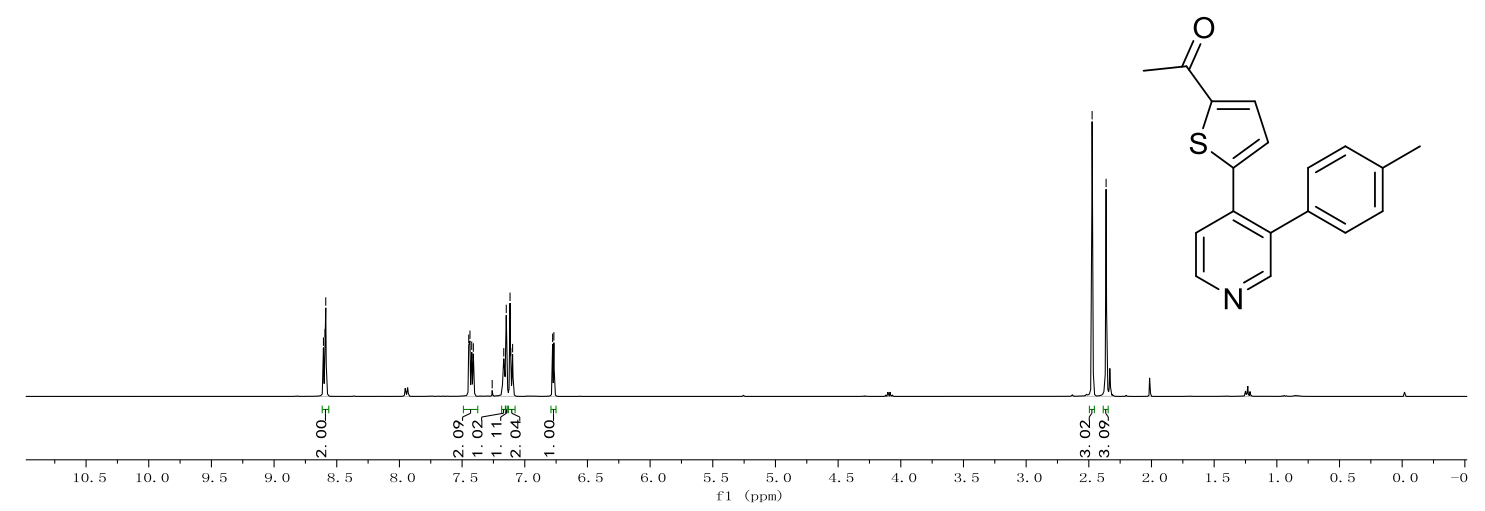

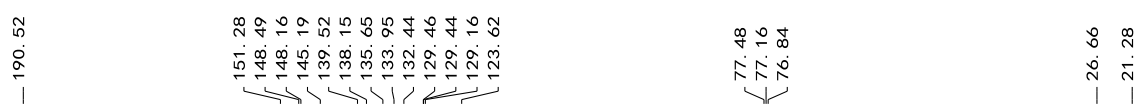

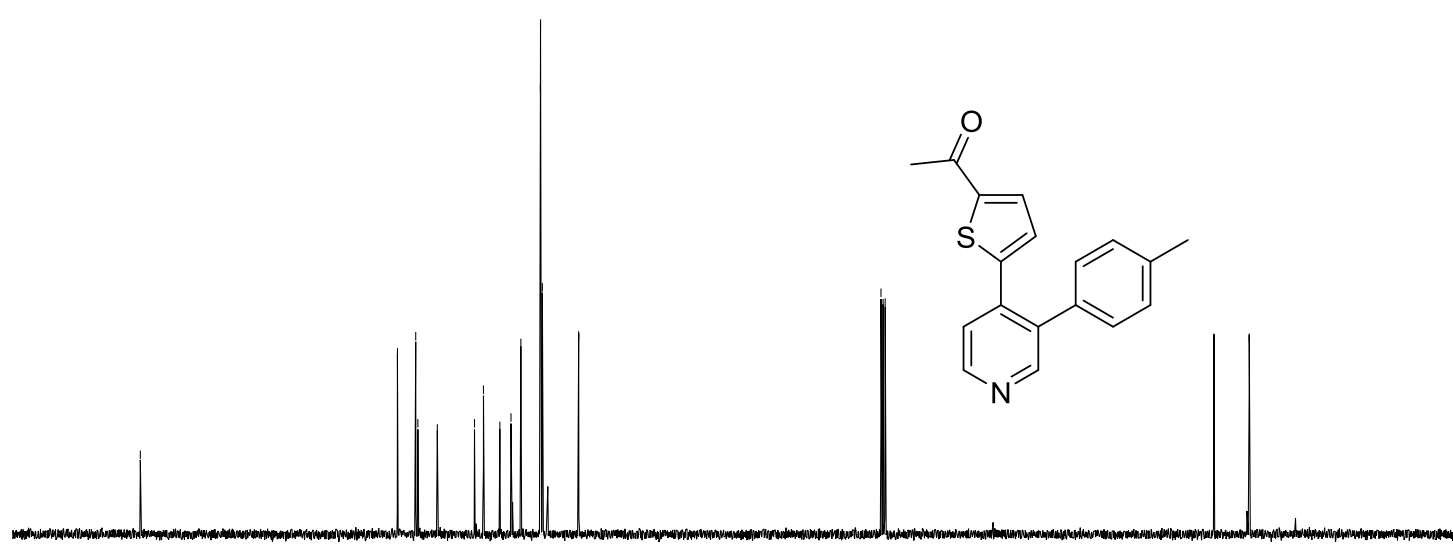

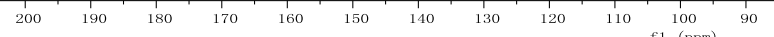


(4h)

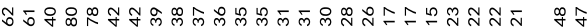

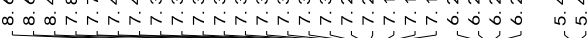

$\stackrel{2}{\circ}$

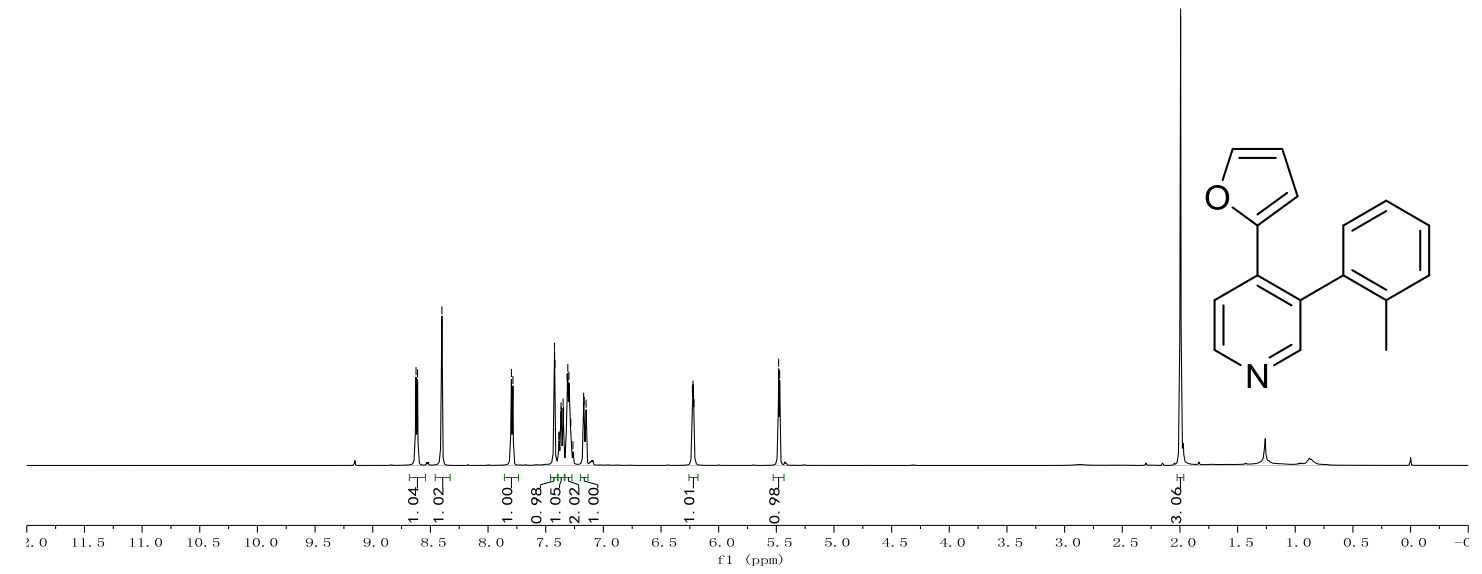

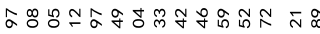

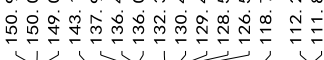

$\stackrel{\infty}{\sim} \stackrel{\infty}{\infty}$

突实

$\frac{1}{2}$

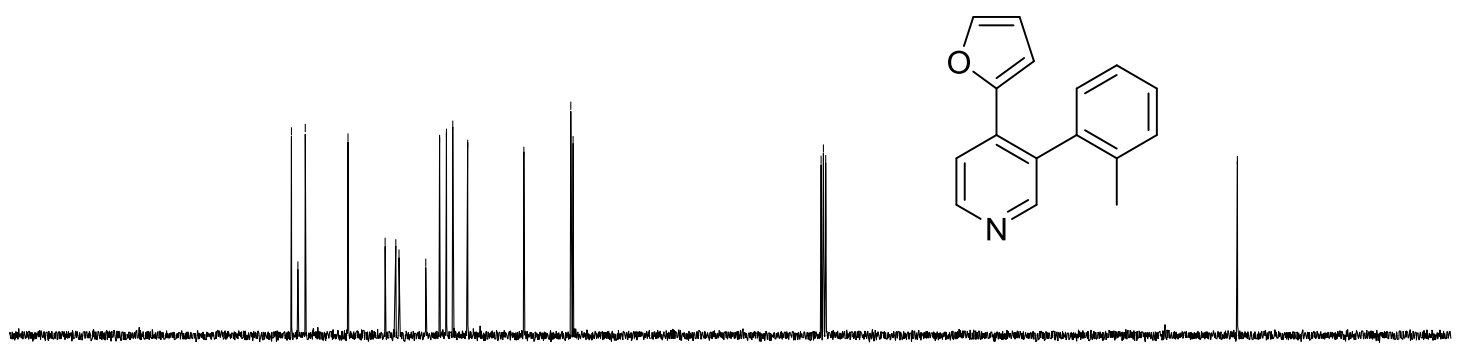

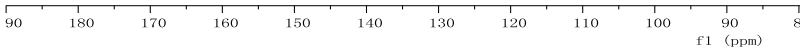



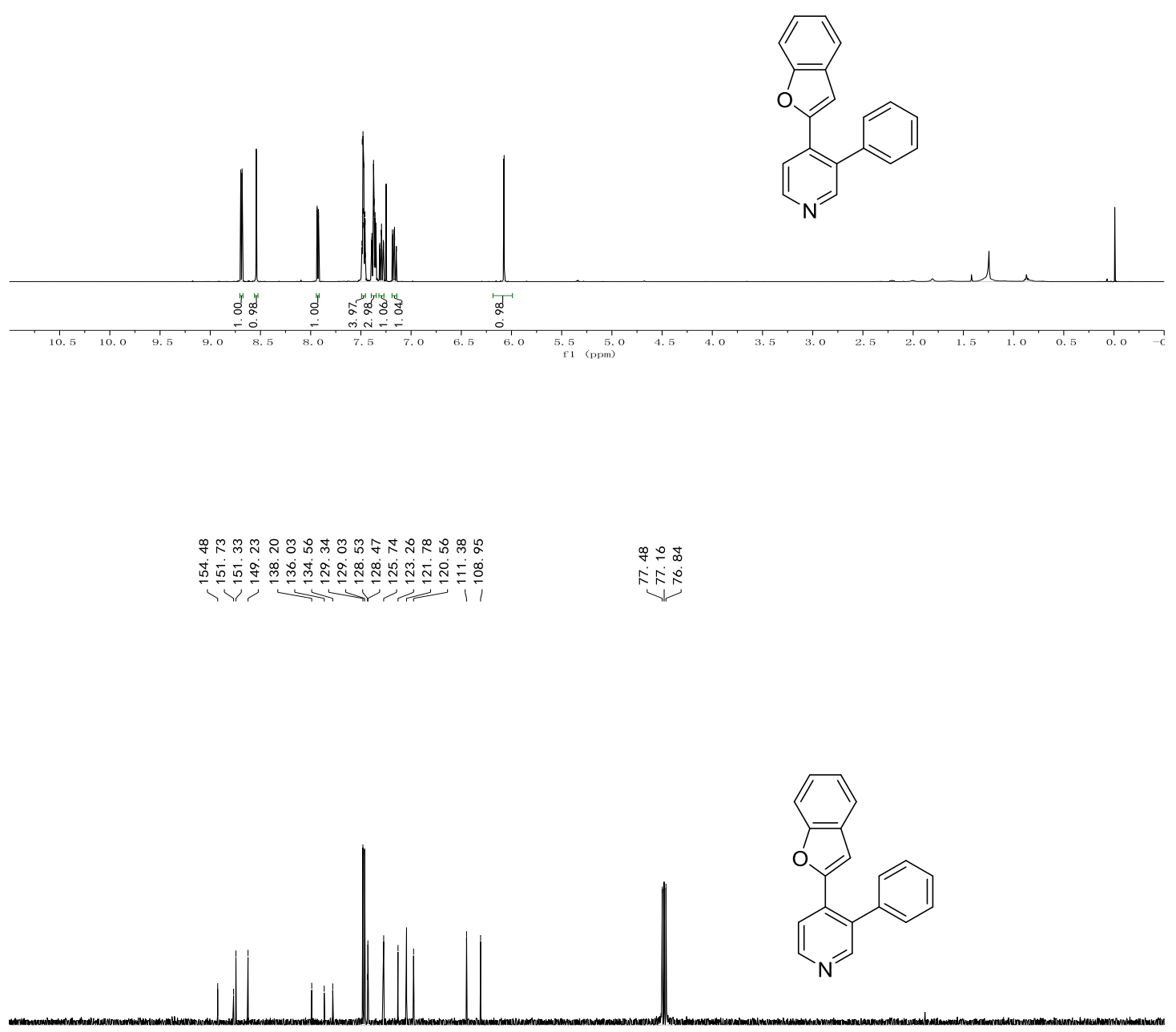
(7a)

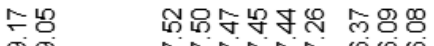

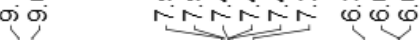

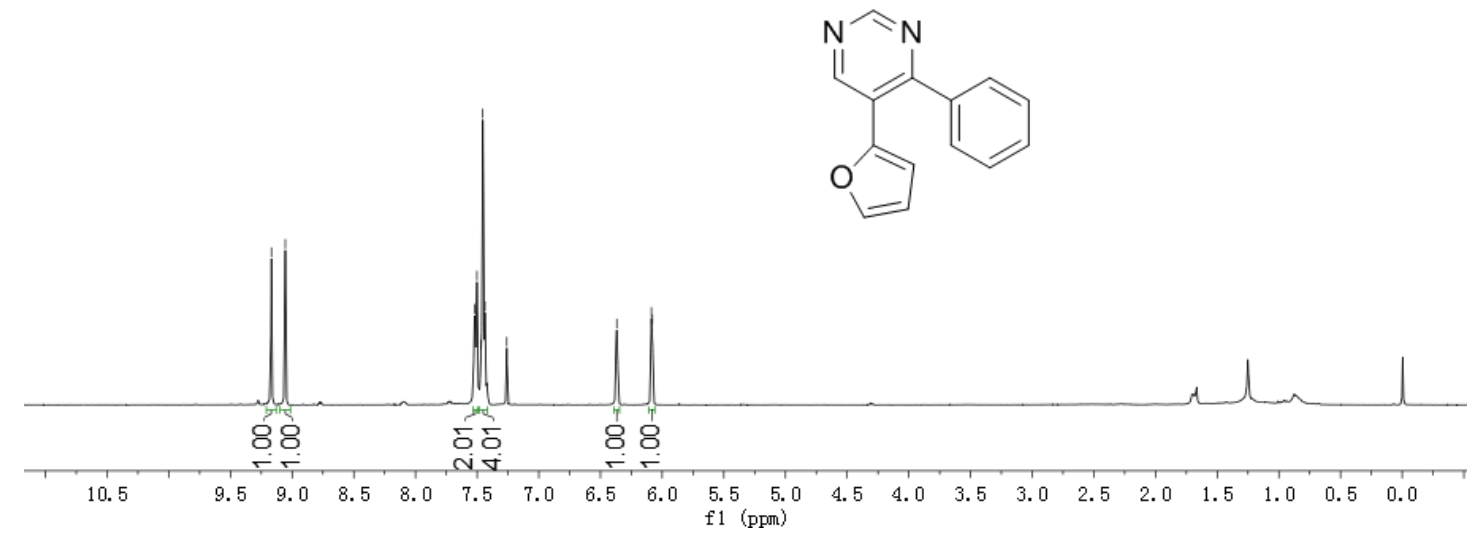

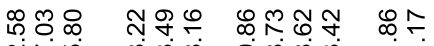

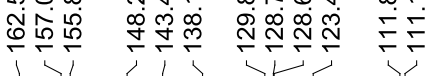

$\stackrel{\infty}{i} \div$

走是

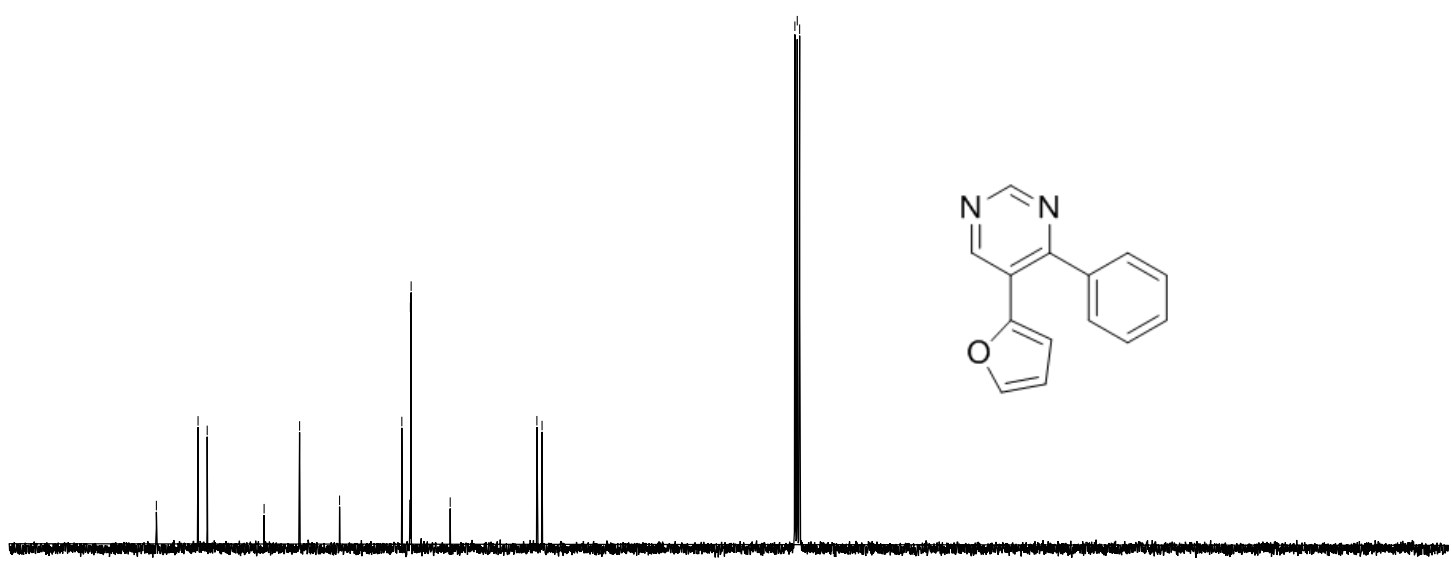

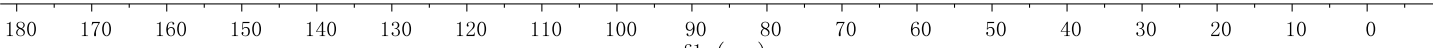


(7b)

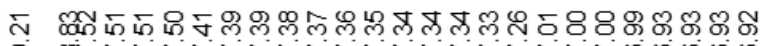

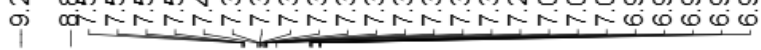
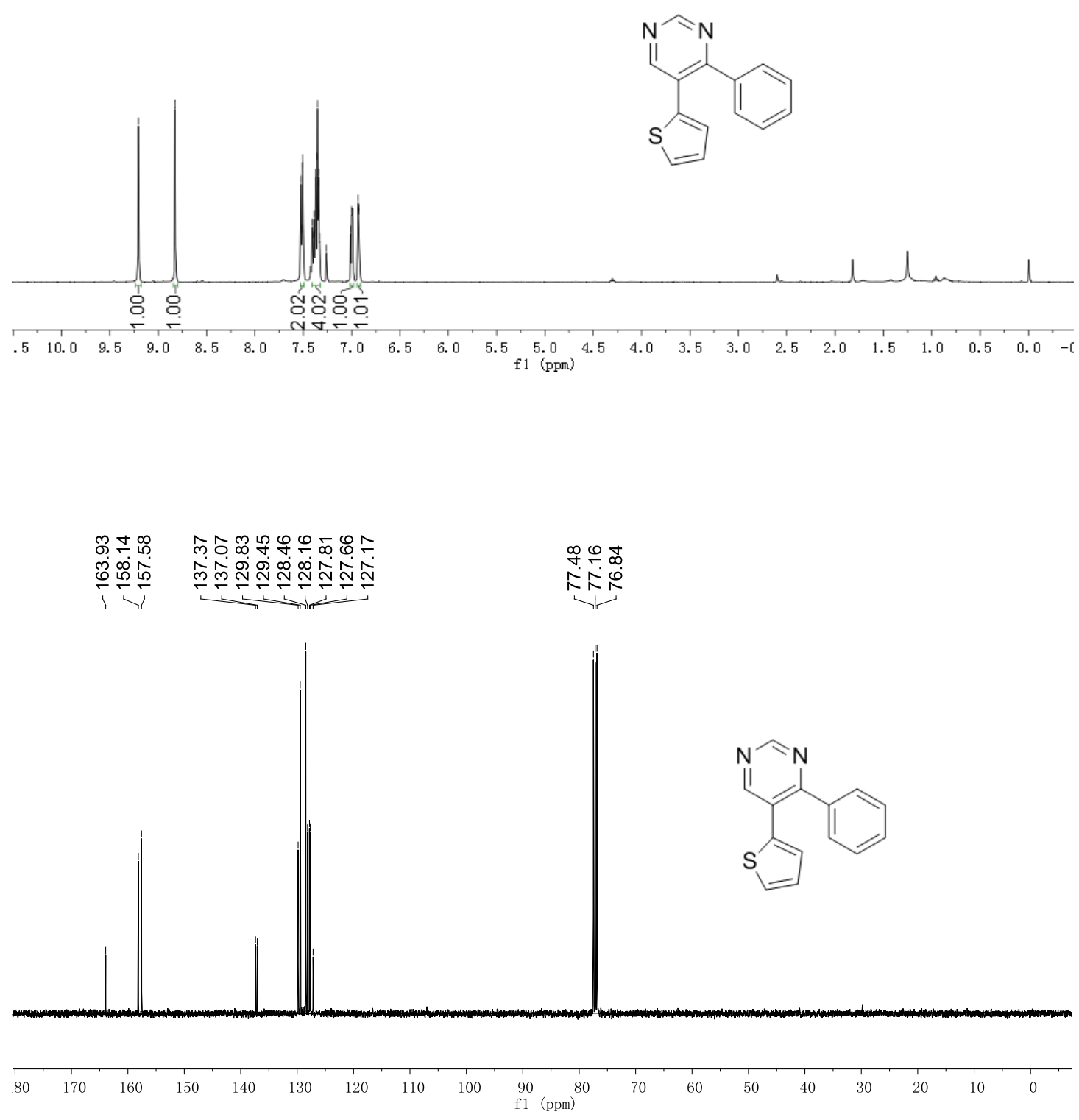

87 
(7c)

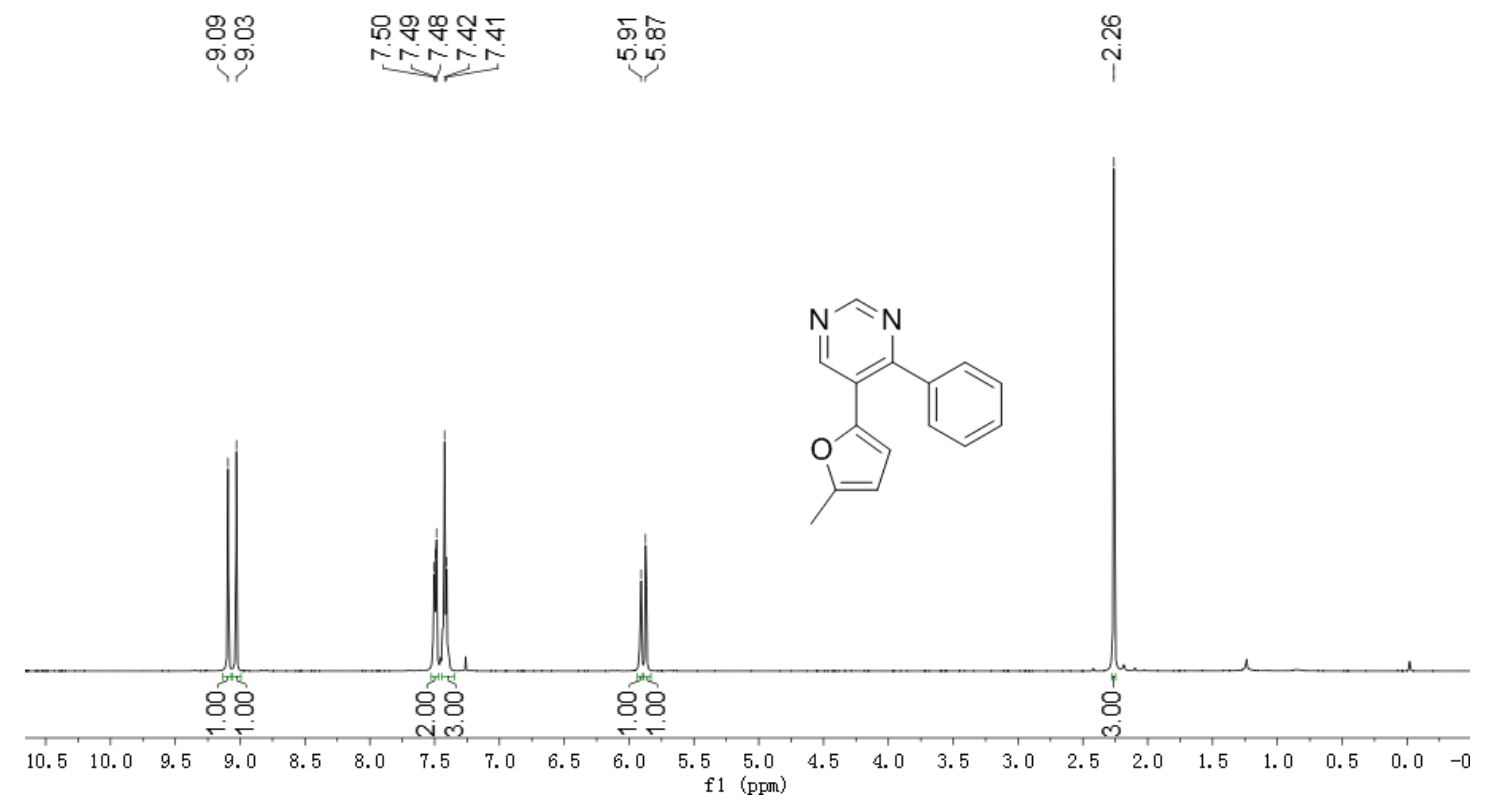

ณ

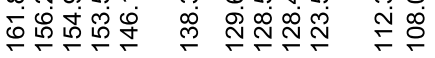

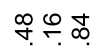

रㅅํㅇ

$\stackrel{\overline{0}}{i}$

|र। | या।

证

$\stackrel{m}{!}$

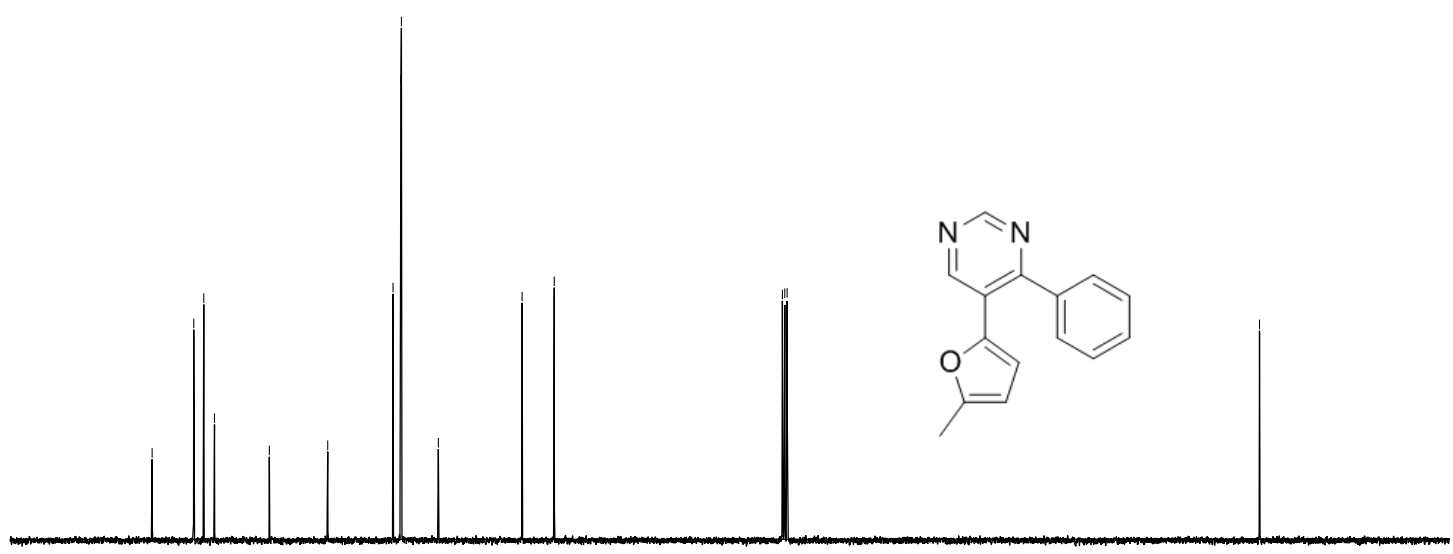

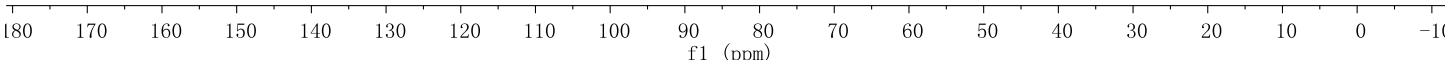


(7d) 일

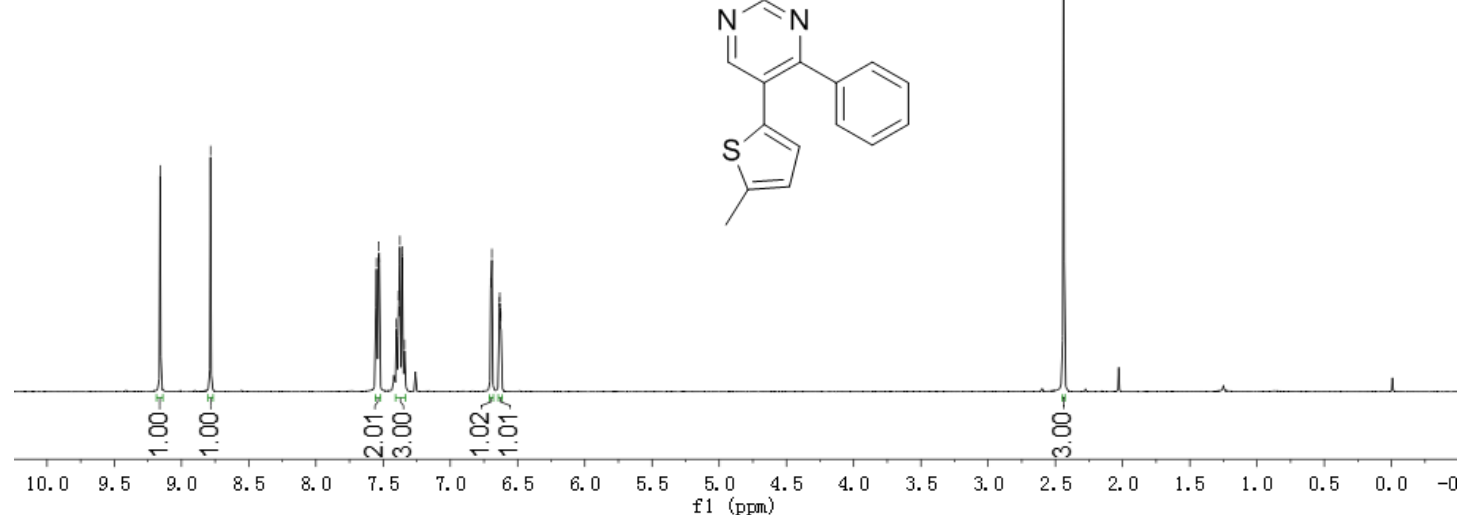

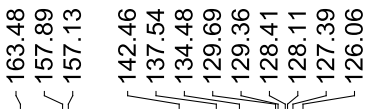

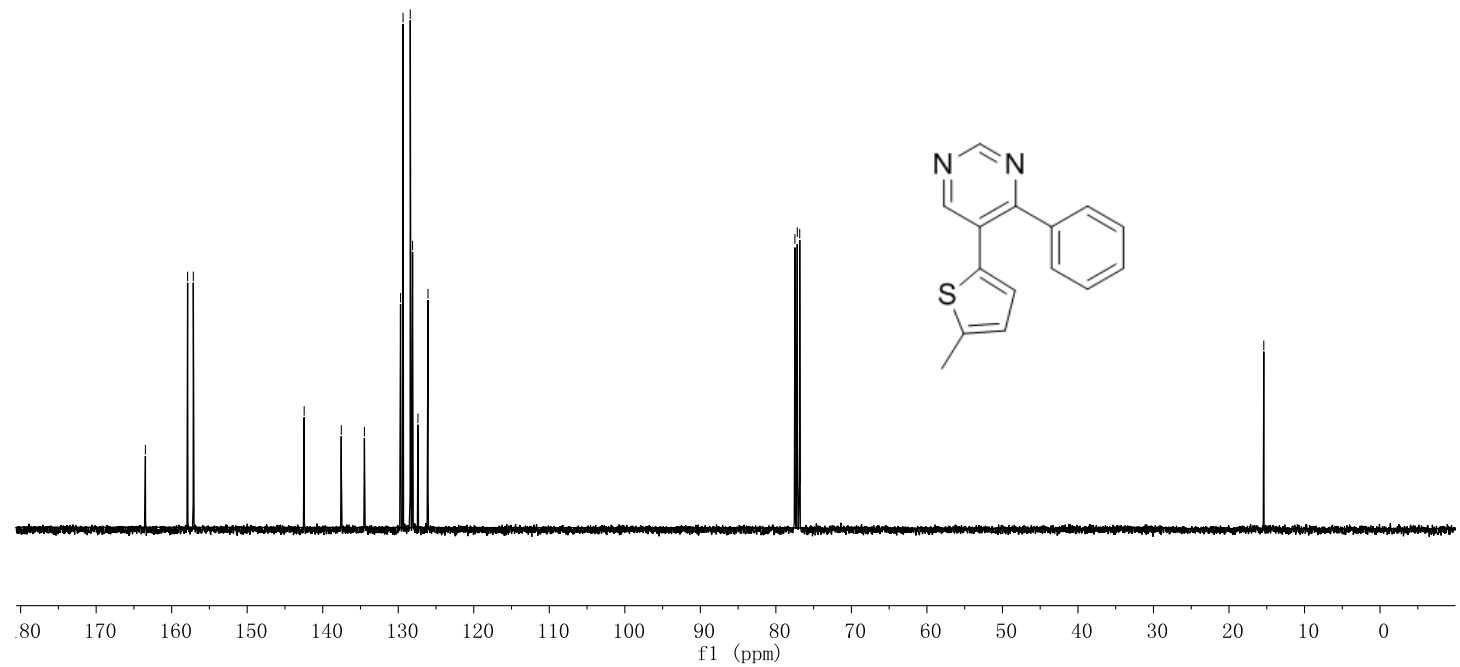

89 
(9a)

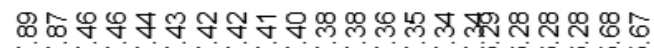

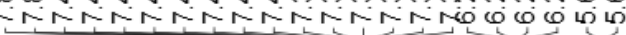

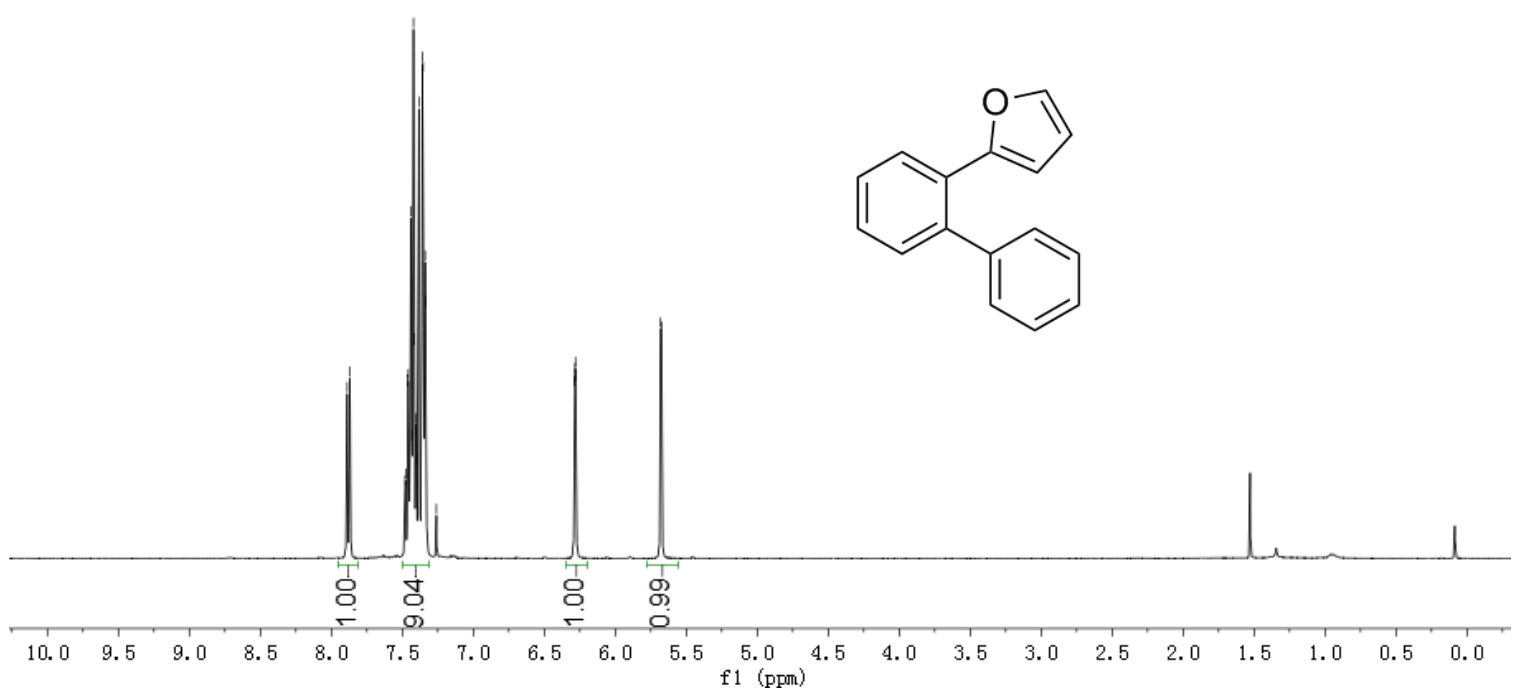

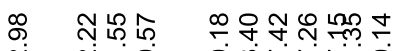

กิ

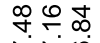

定完

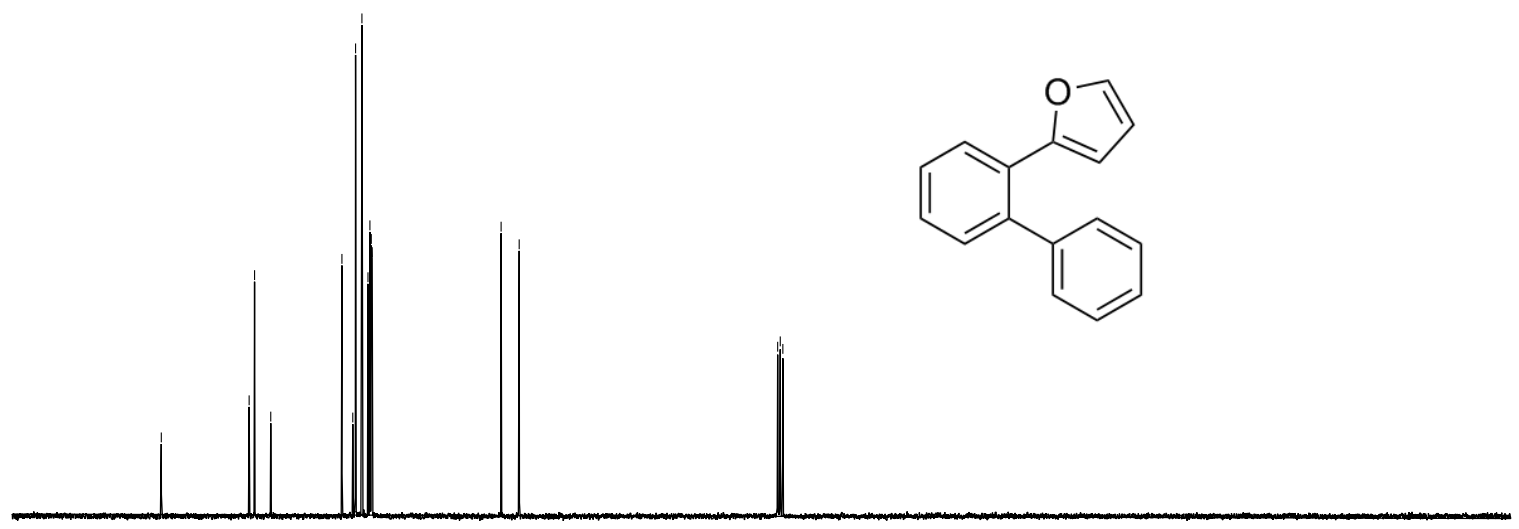

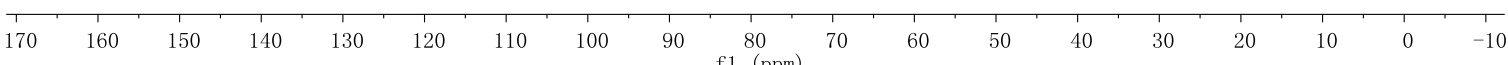


The spectra of $2 a-2 v, 5 a-5 d, 6 a-6 j, 8 a-8 d$

(2a)

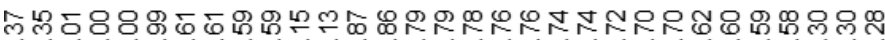

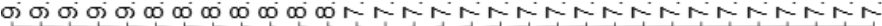

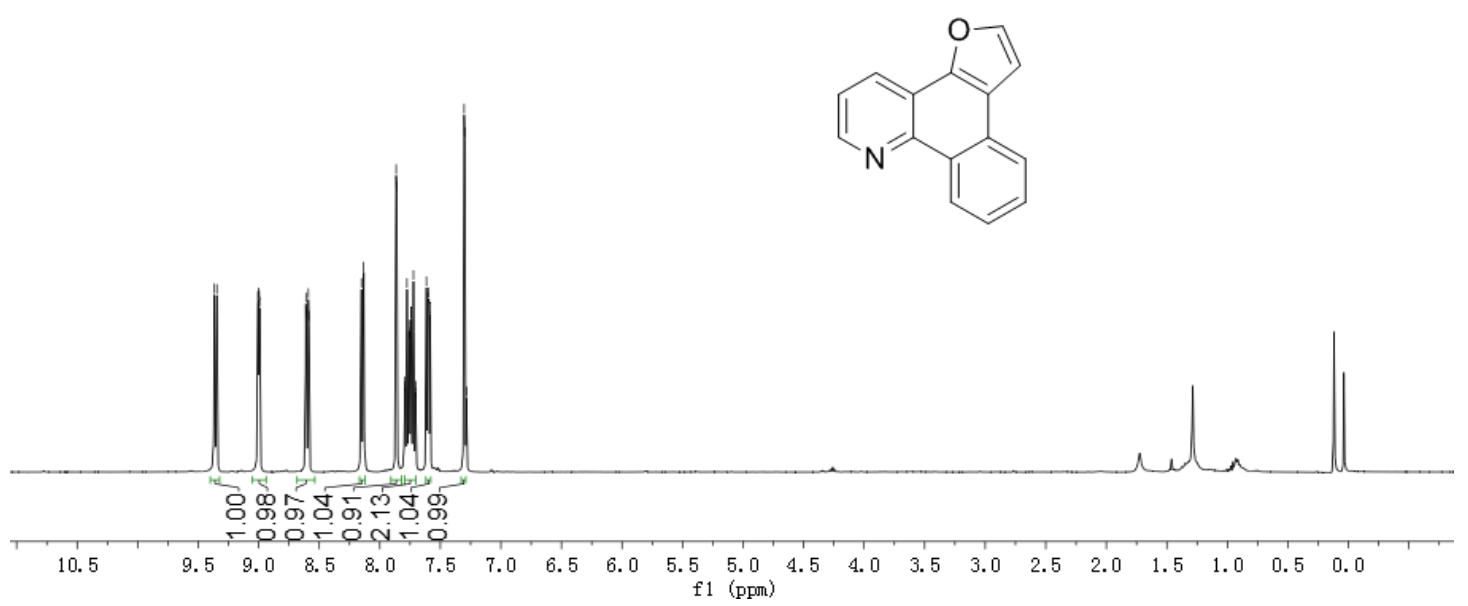

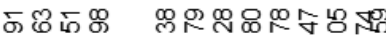

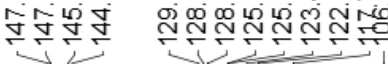

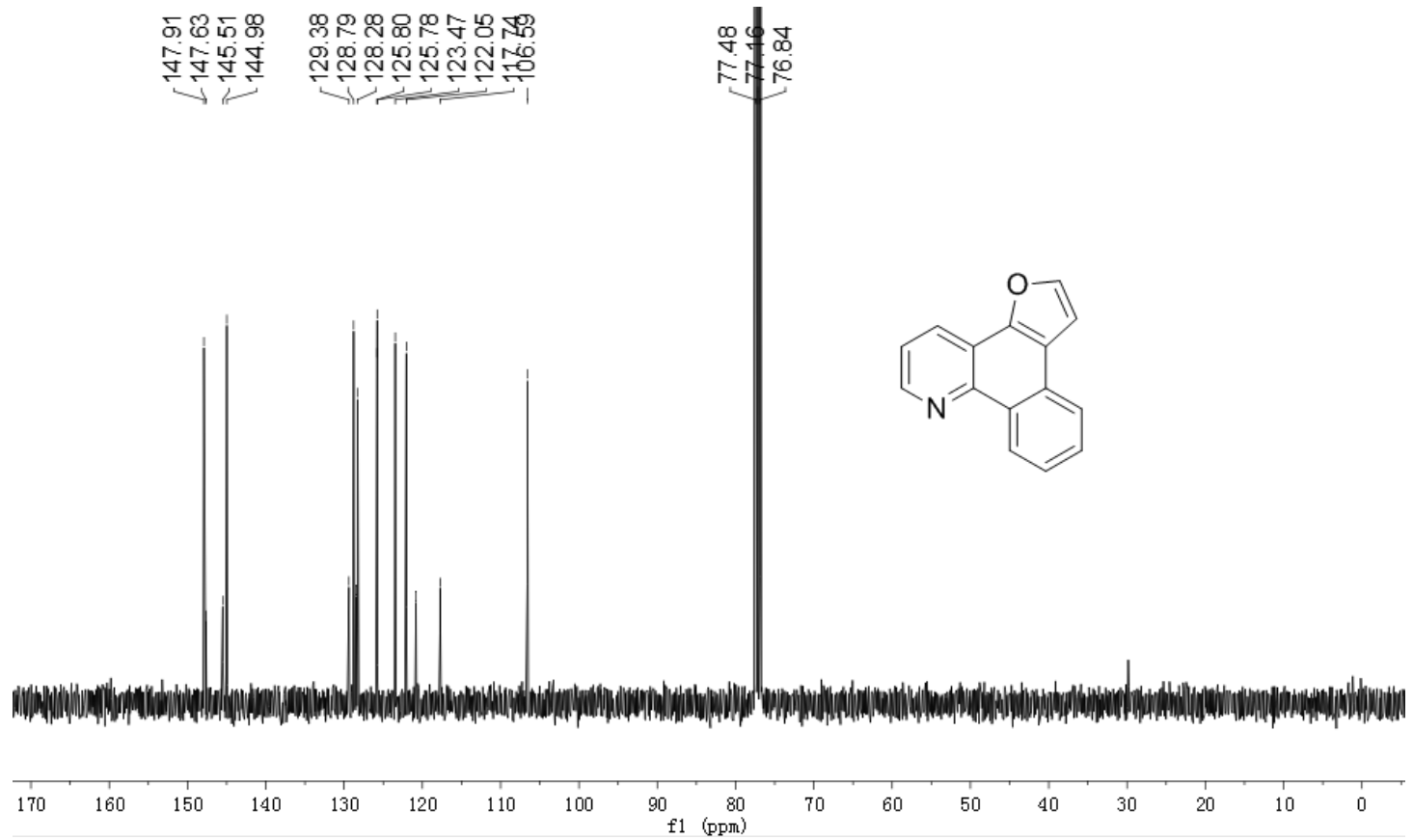


(2b)

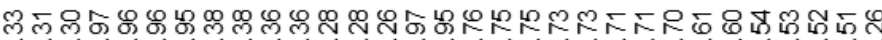
Oj,
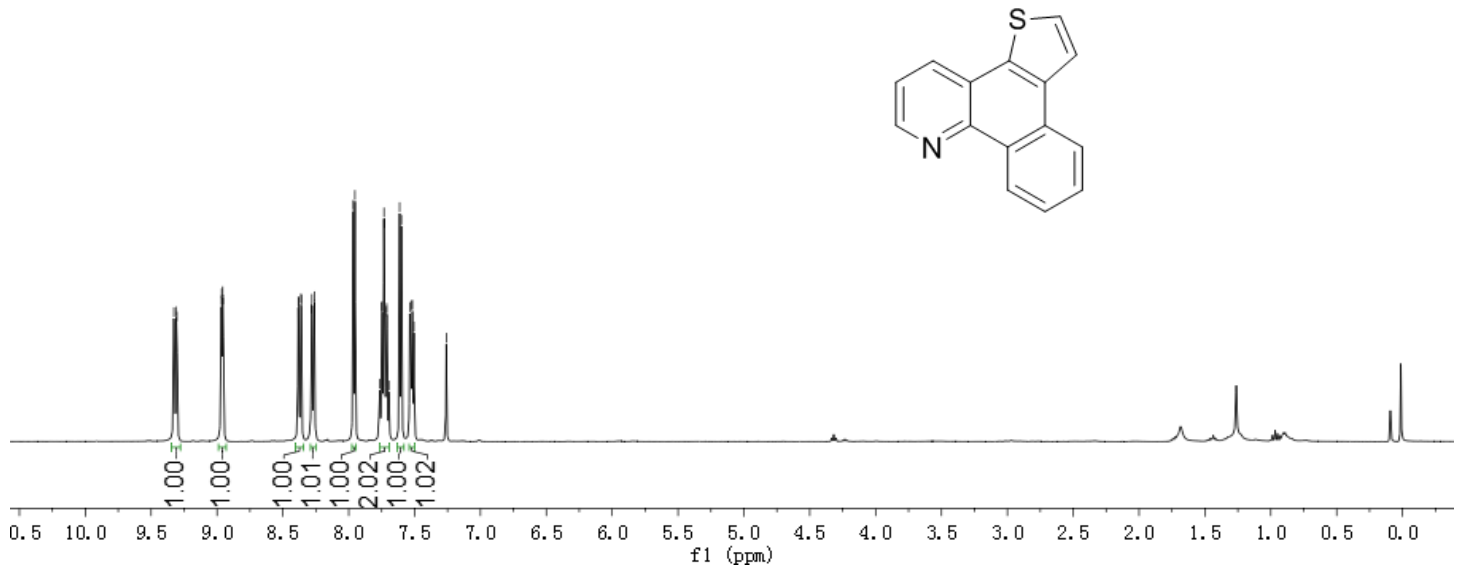

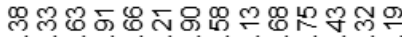

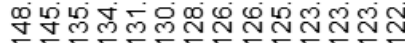
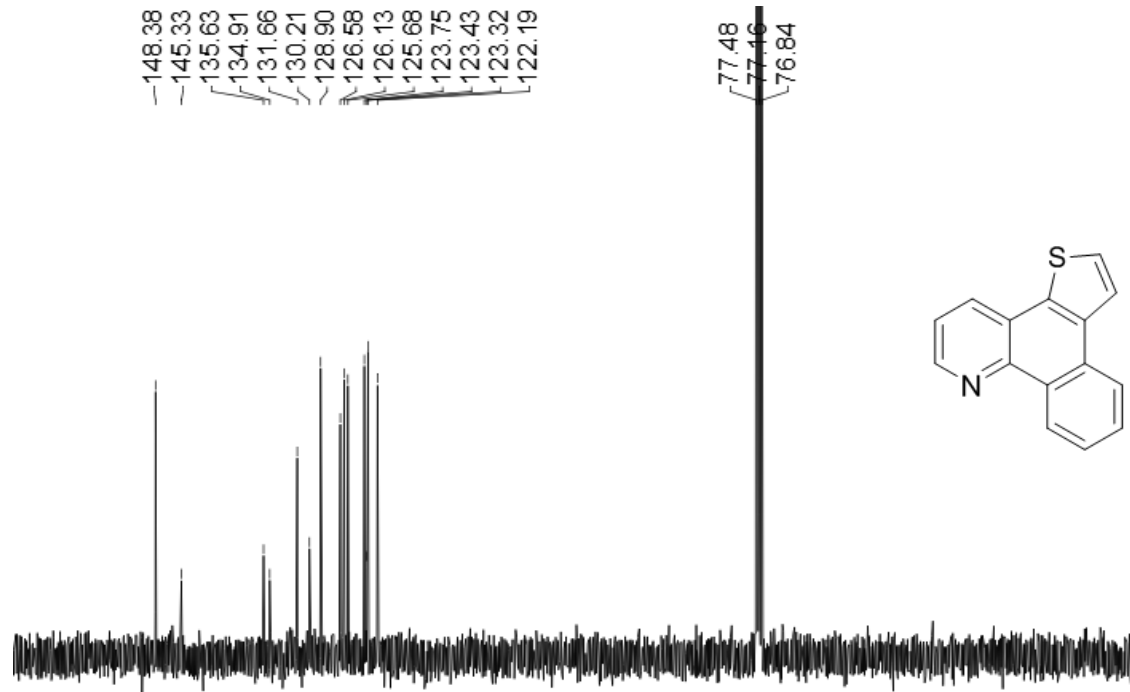

$\begin{array}{lllllllllllllllll}160 & 150 & 140 & 130 & 120 & 110 & 100 & 90 & \underset{\mathrm{f} 1}{8}(\mathrm{ppm}) & 70 & 60 & 50 & 40 & 30 & 20 & 10 & 0\end{array}$


(2c)<smiles>Cc1cc2c3ccccc3c3ncccc3c2s1</smiles>

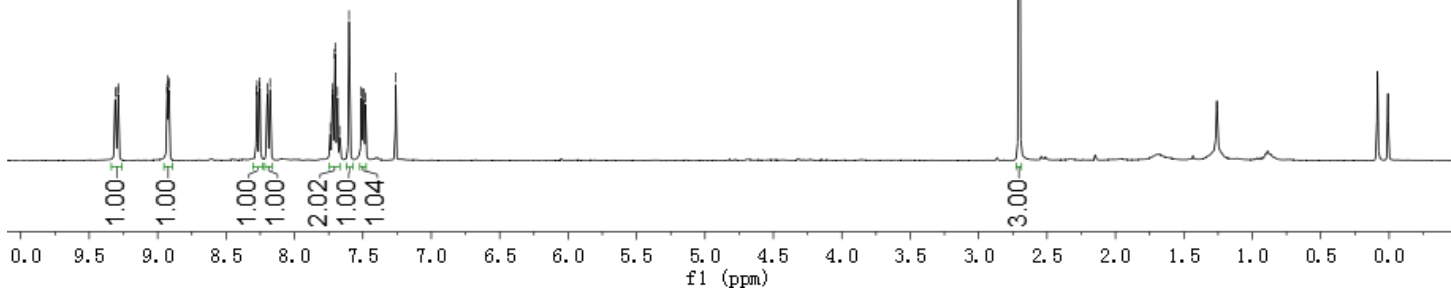

๓

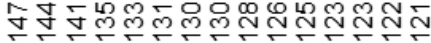
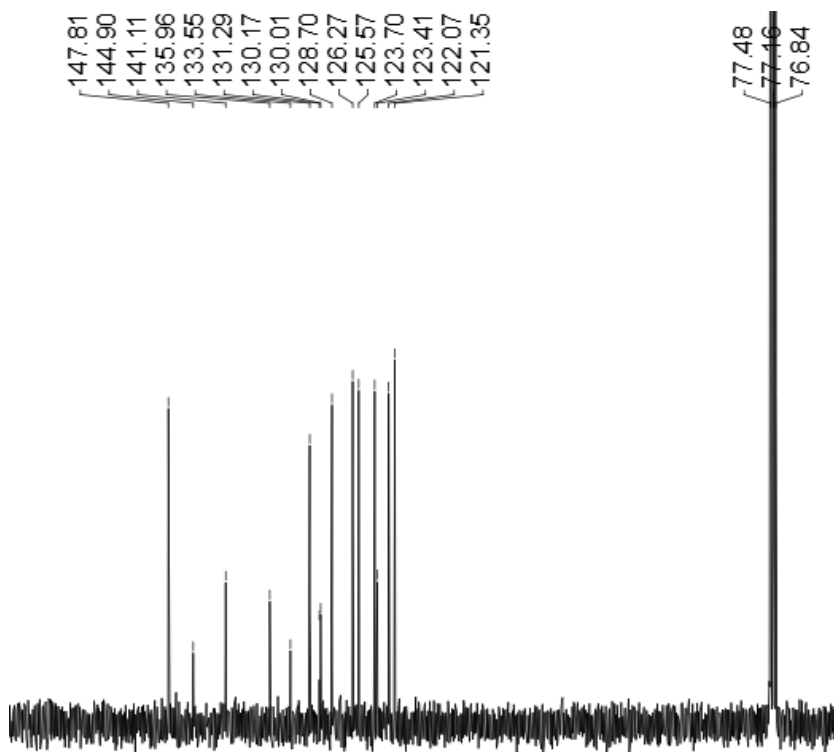<smiles>Cc1cc2c3cccnc3c3ccccc3c2s1</smiles>

$\stackrel{\infty}{\infty}$

$\begin{array}{llll}160 & 150 & 140 & 130\end{array}$

f1 $\stackrel{80}{(\mathrm{ppm})}$ 
(2d)

ำำ

$\stackrel{\circledast}{\leftrightarrow}$
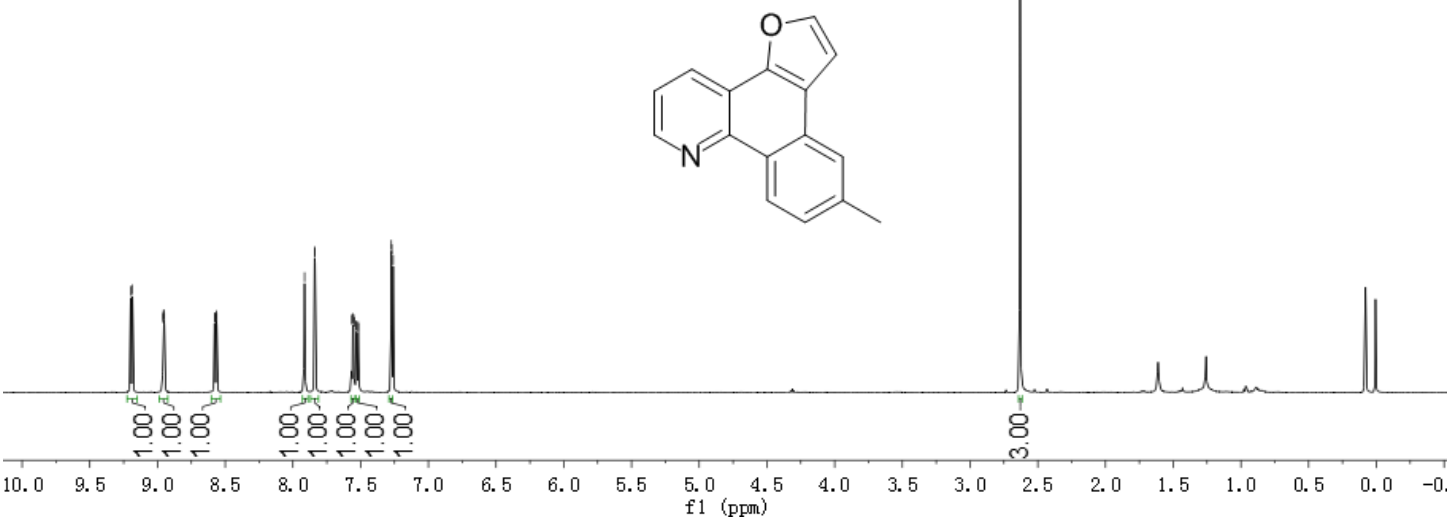

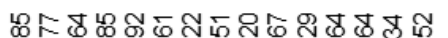

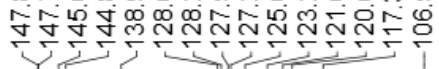

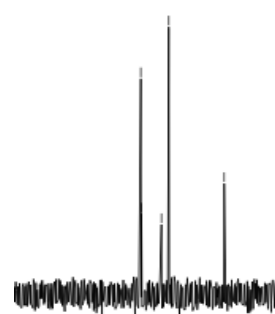

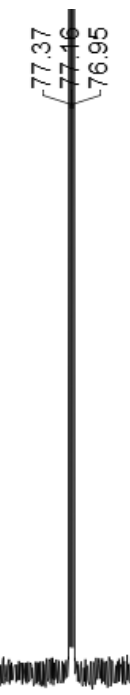

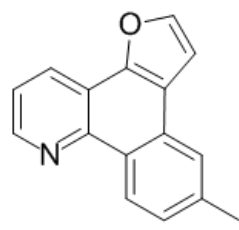

$\stackrel{\text { \% }}{\stackrel{\mathrm{T}}{\mathrm{T}}}$

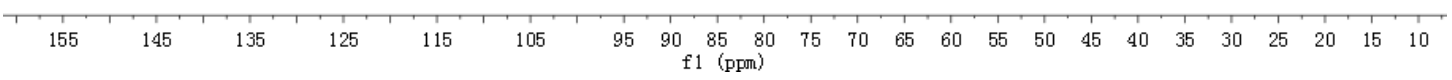

94 
(2e)

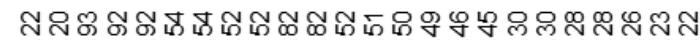

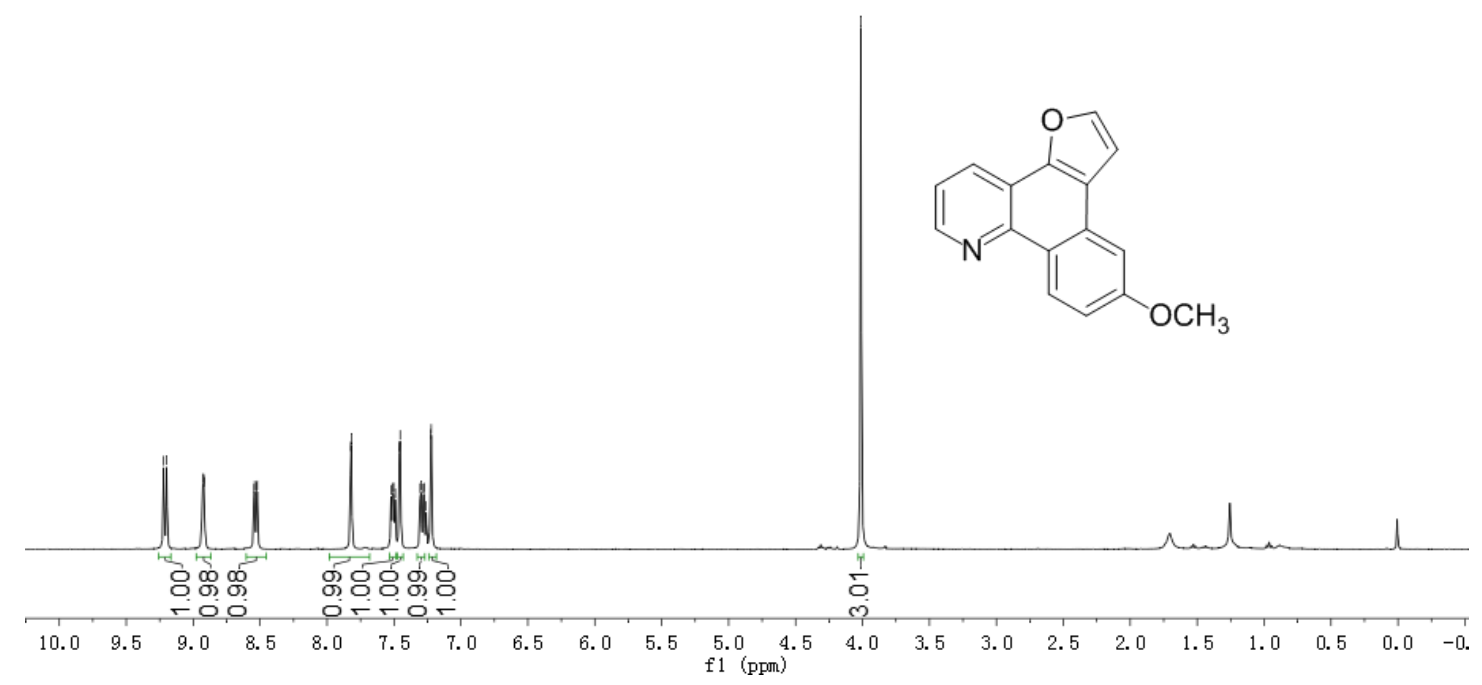

m ㅇำ

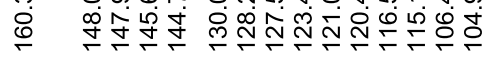

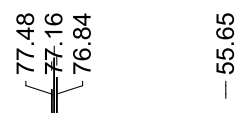

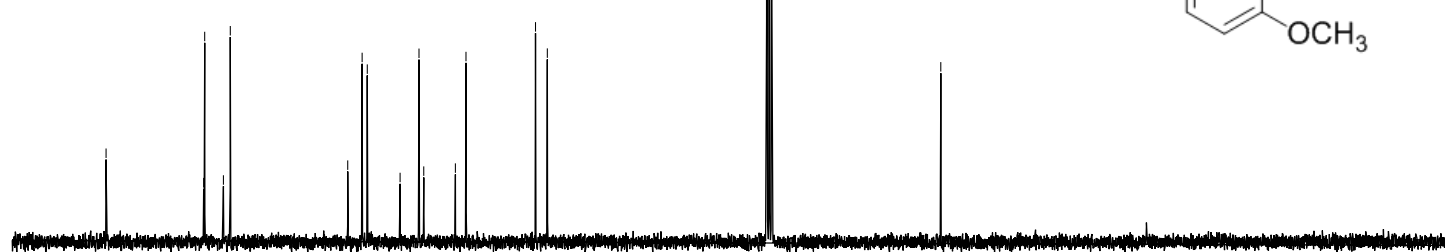

170

$\begin{array}{llll}160 & 150 & 140 & 130\end{array}$

$120 \quad 110$

100

90

f1 $\begin{gathered}80 \\ (\mathrm{ppm})\end{gathered}$

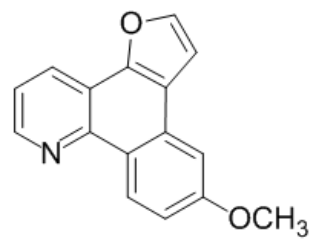



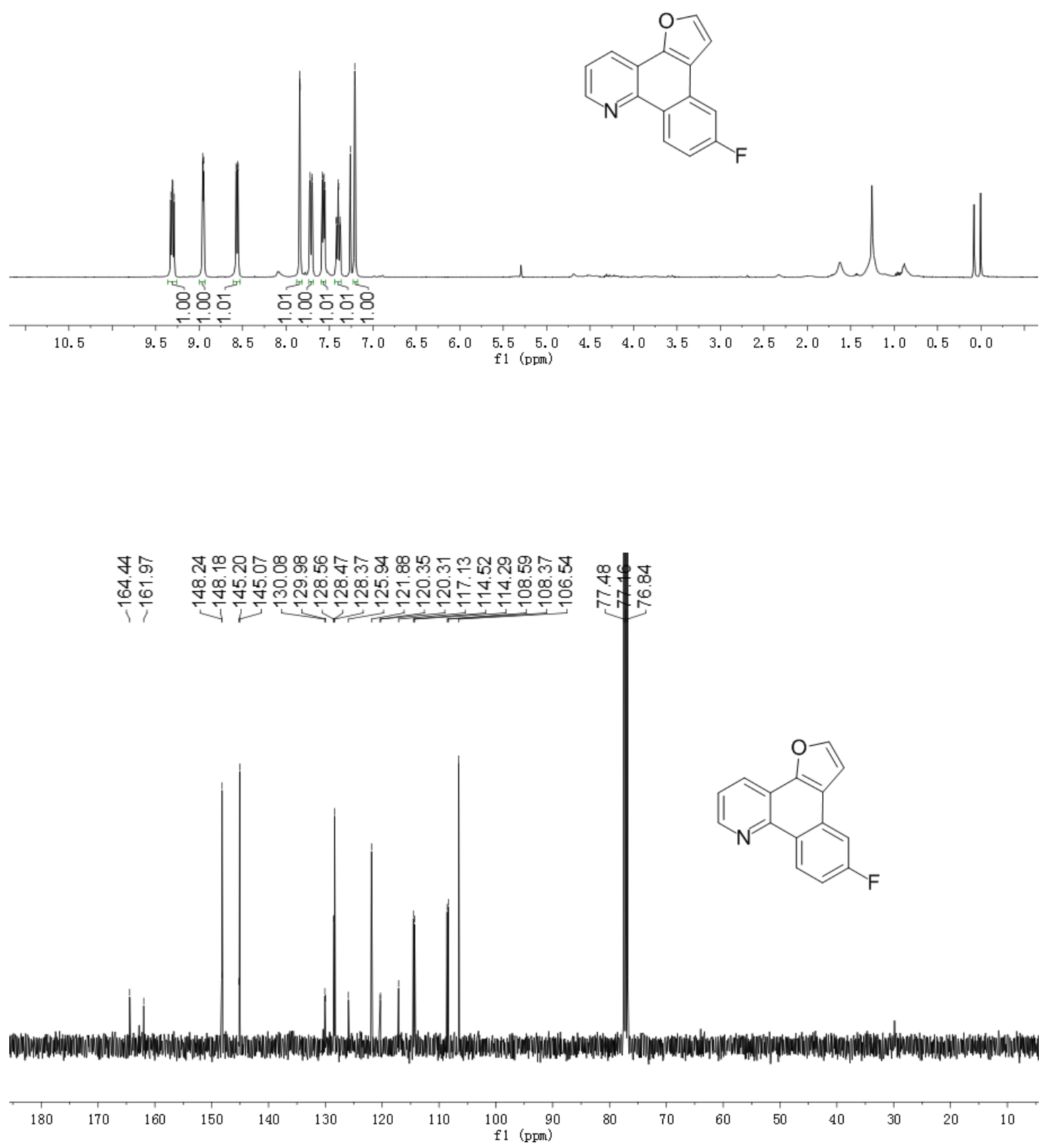
$(2 \mathrm{~g})$
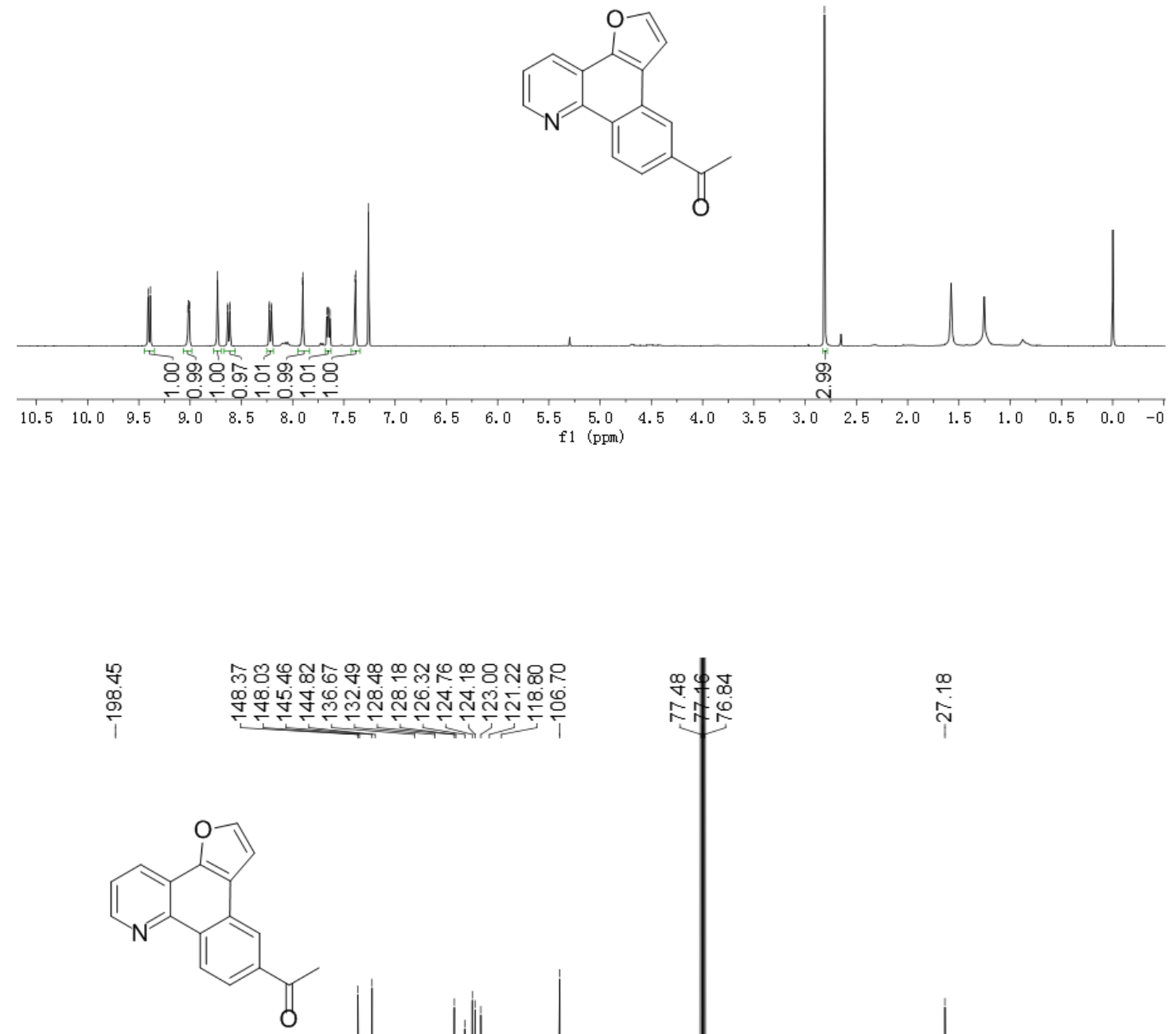

m.

嵅守守

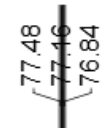

$\stackrel{\infty}{\stackrel{\infty}{\Gamma}}$
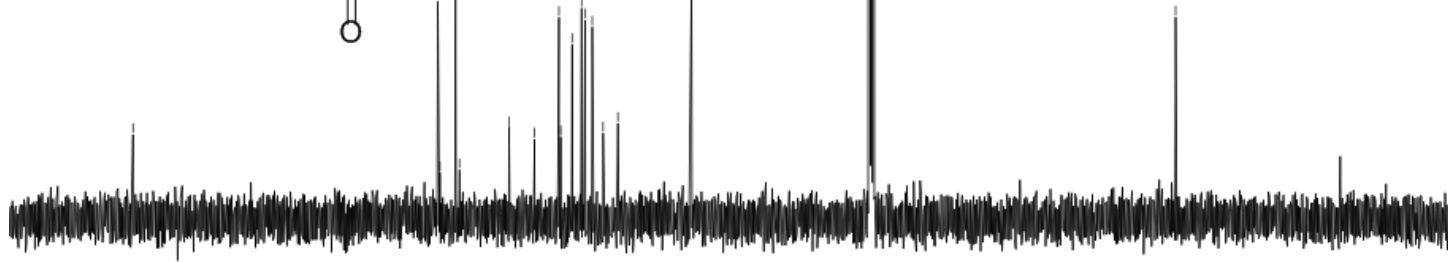

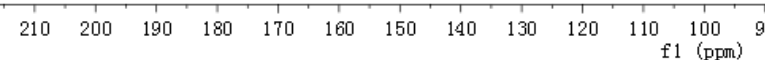


(2h)

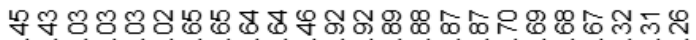

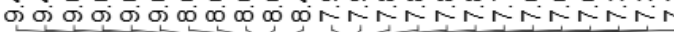
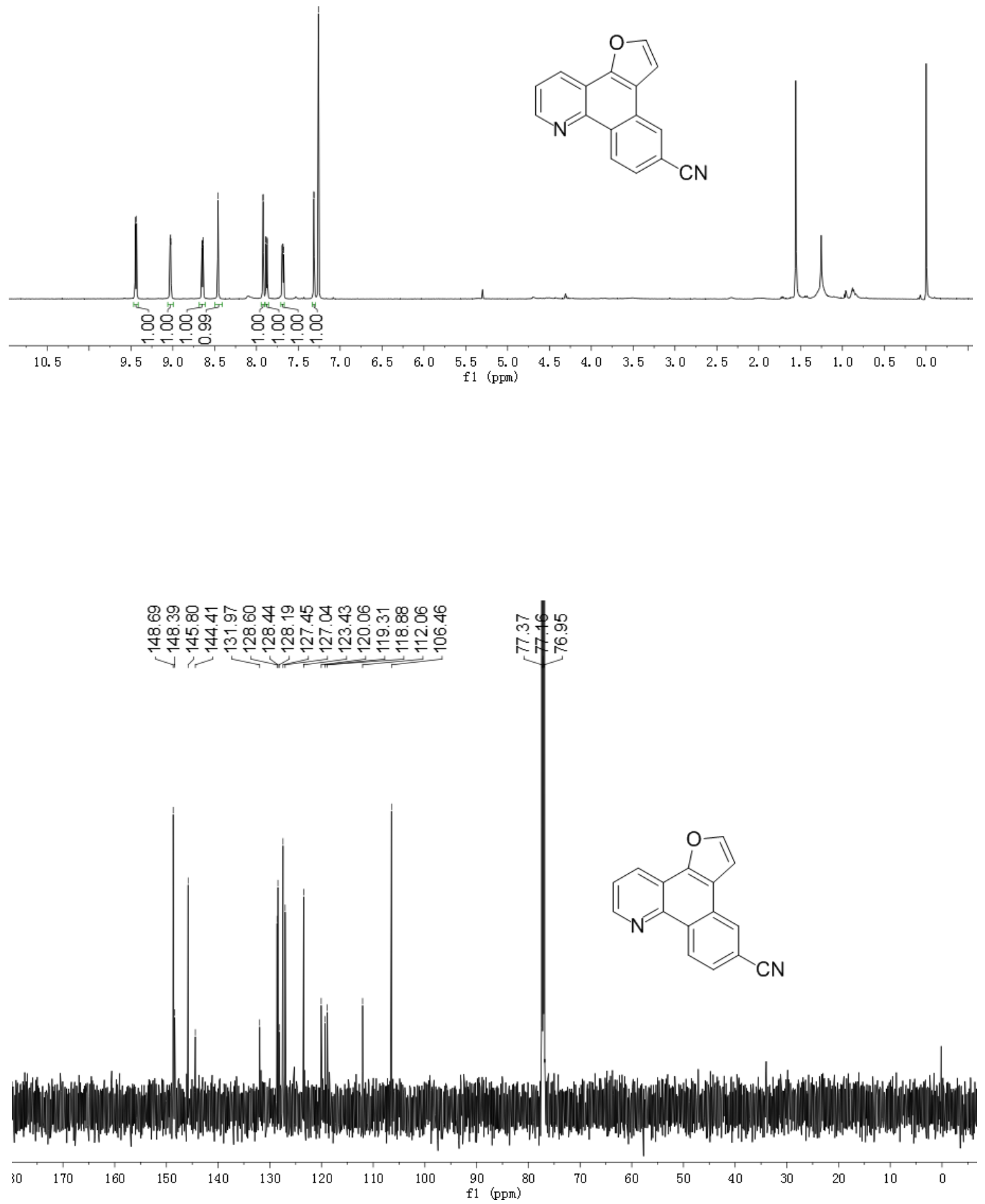


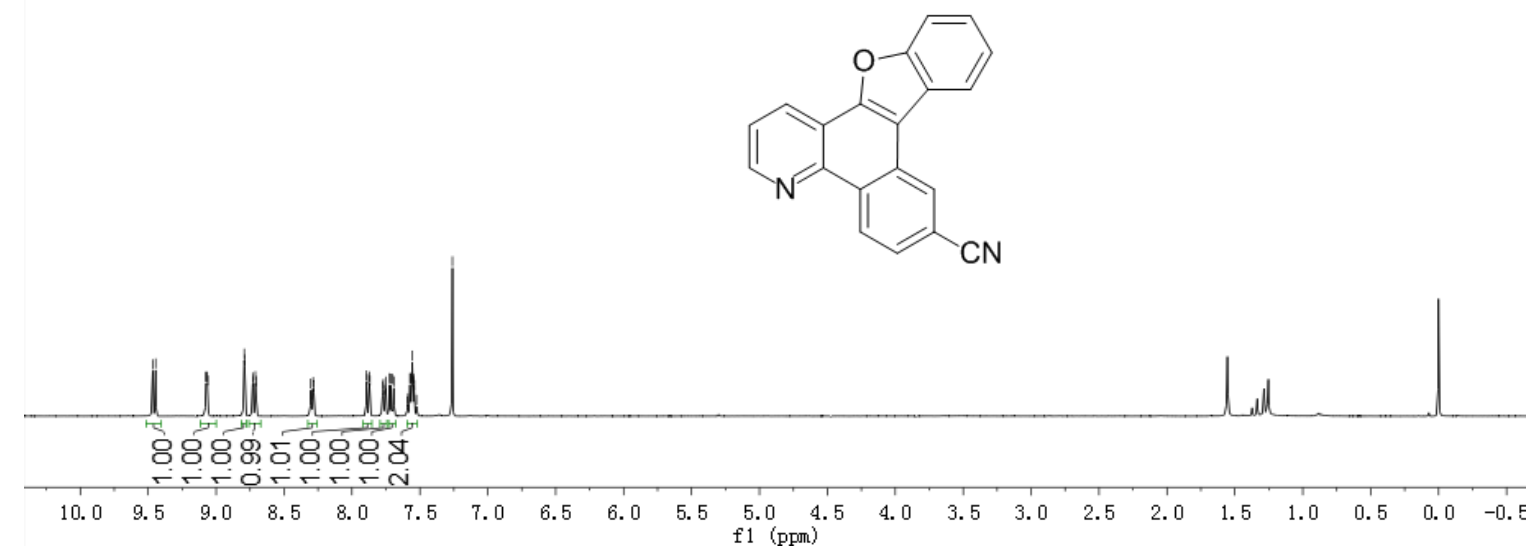

ด

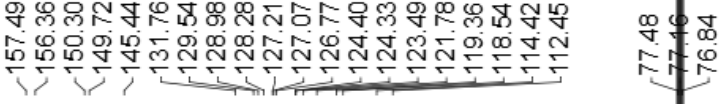

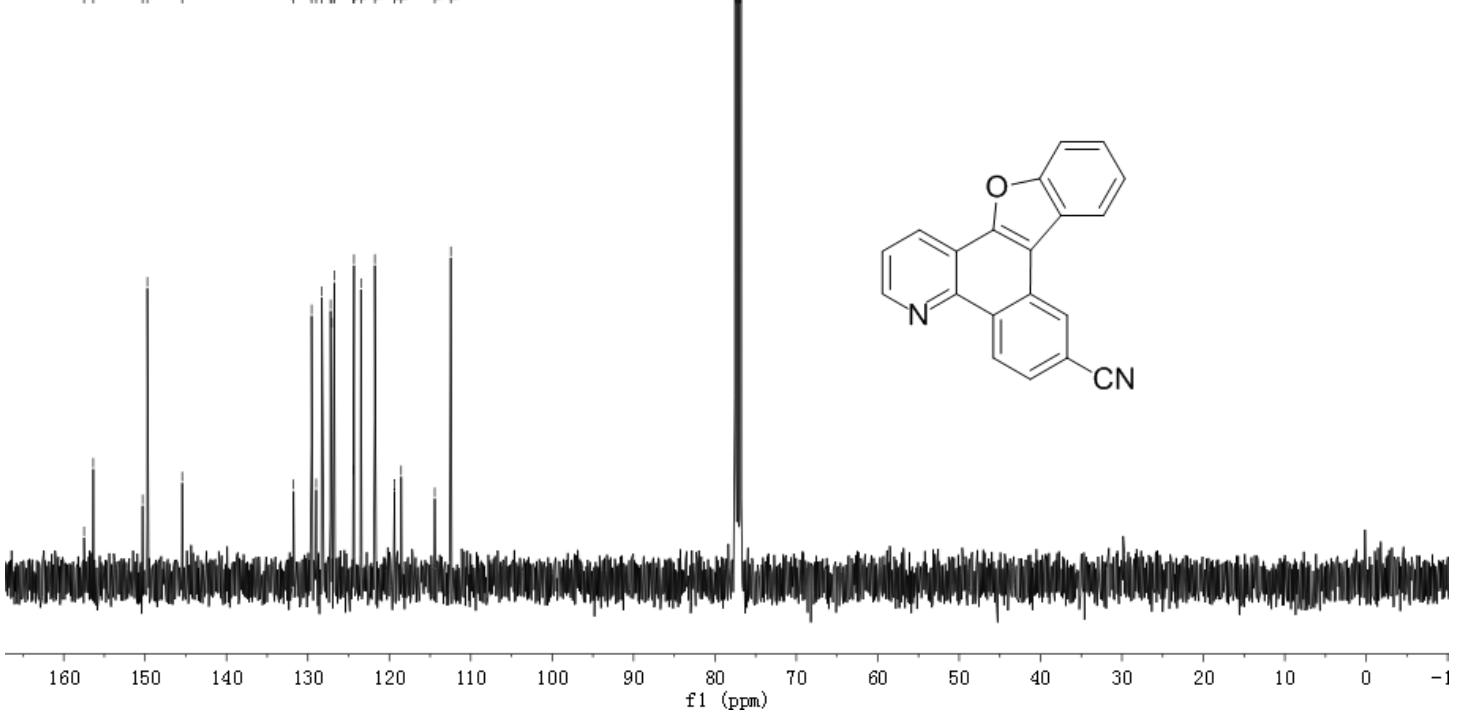


(2j)

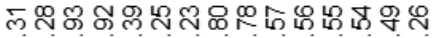

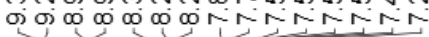

$\underset{1}{i}$
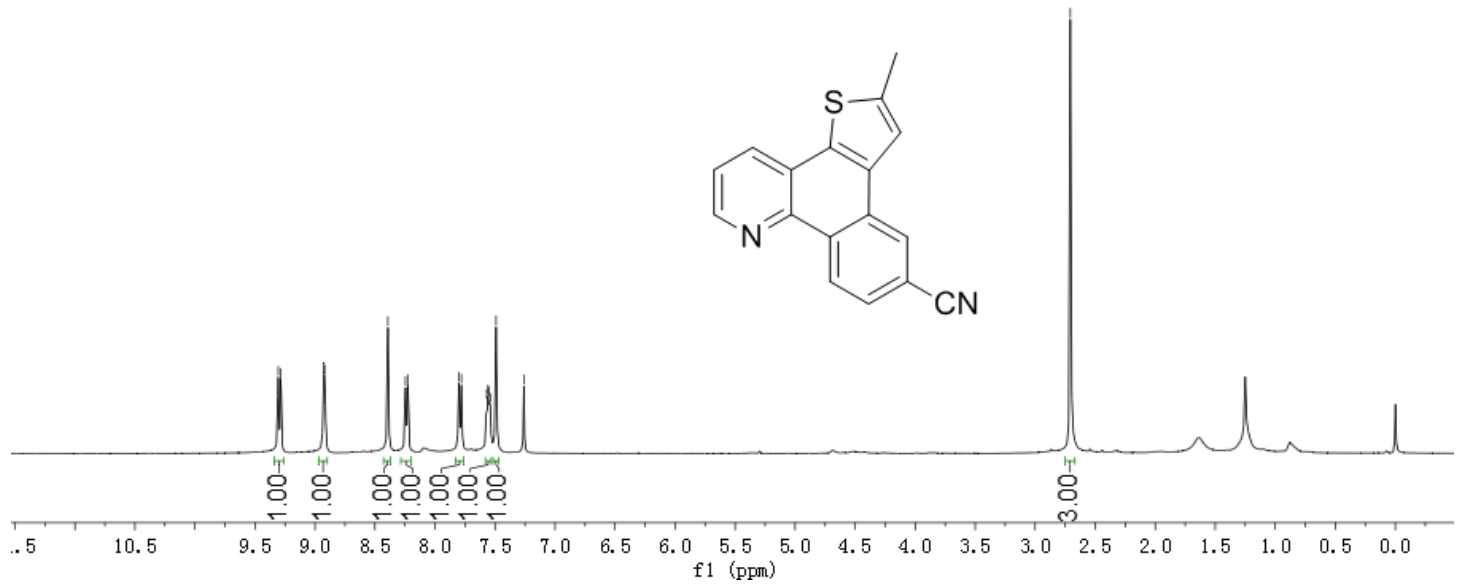

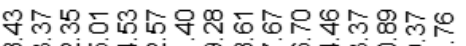

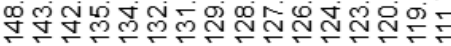

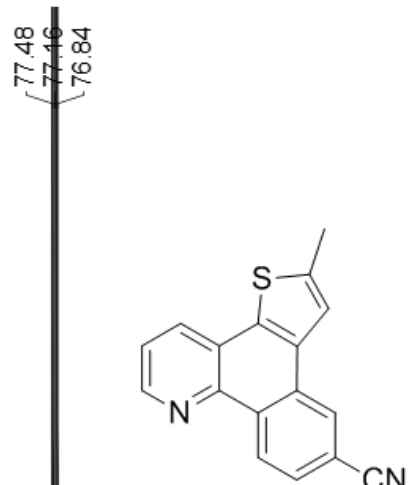

$\stackrel{\substack{\infty \\ \oplus}}{\stackrel{\omega}{\varphi}}$

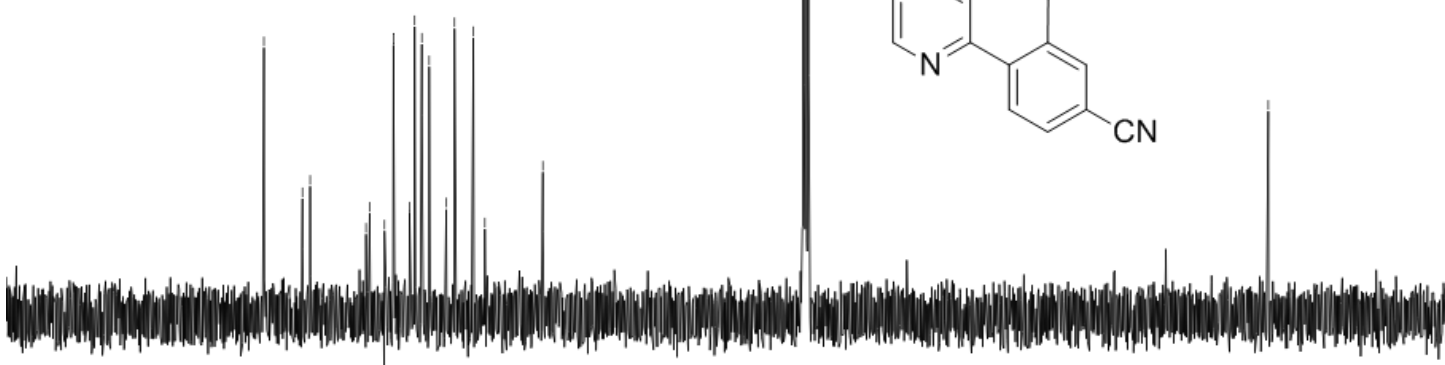

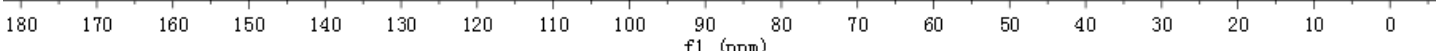


(2k)

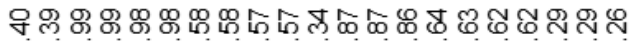
o
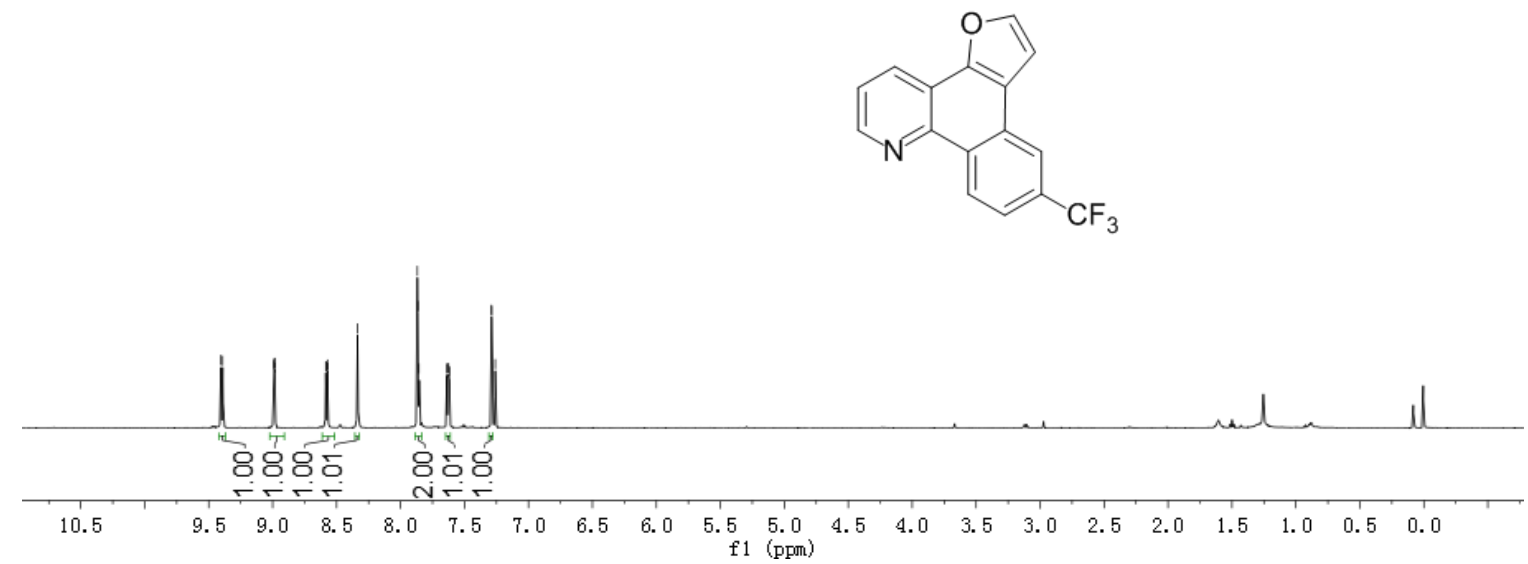

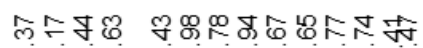

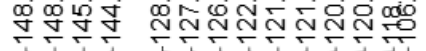

f1 (ppm)
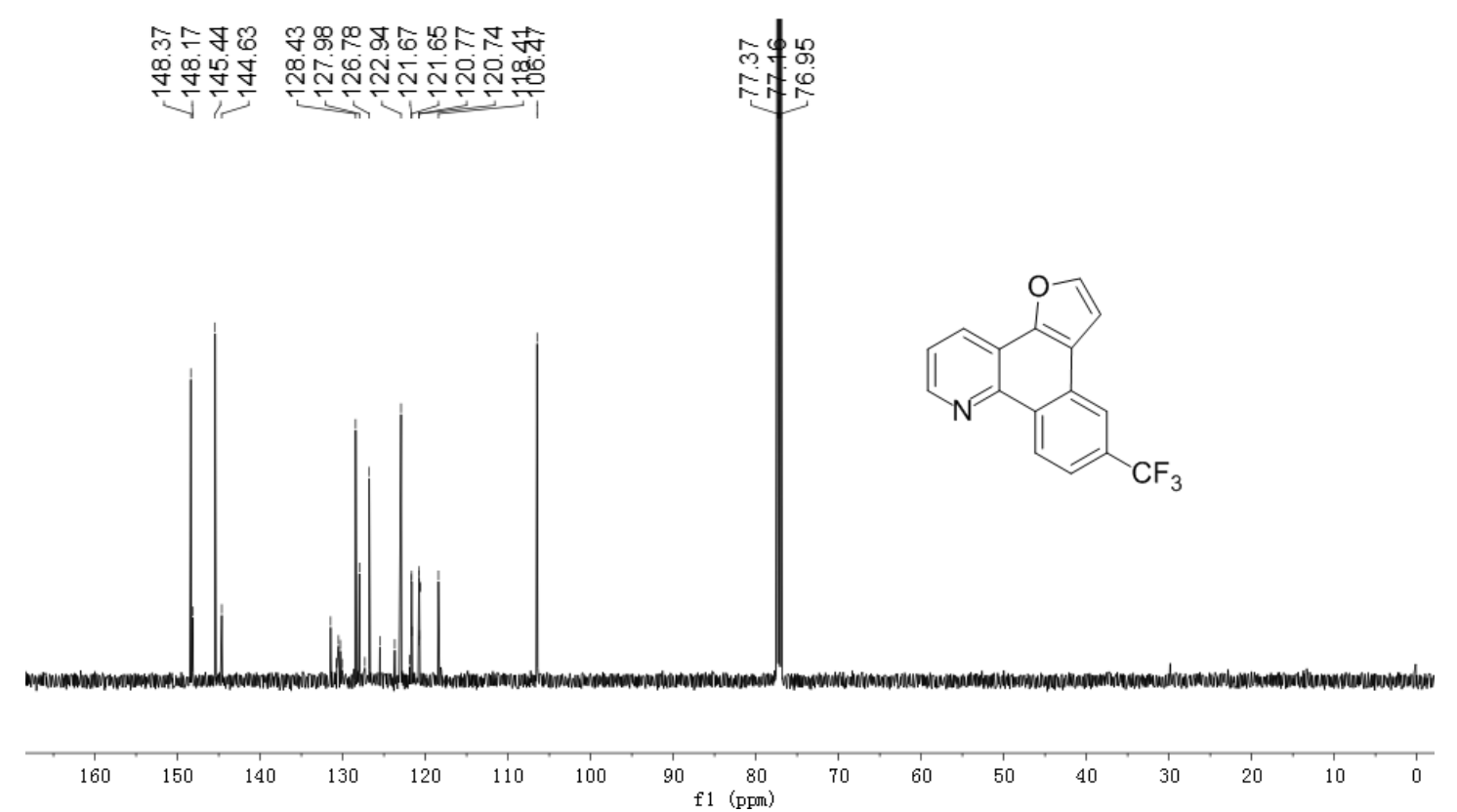
(2I)

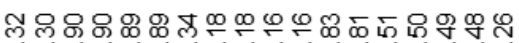

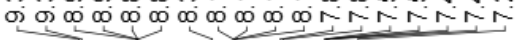
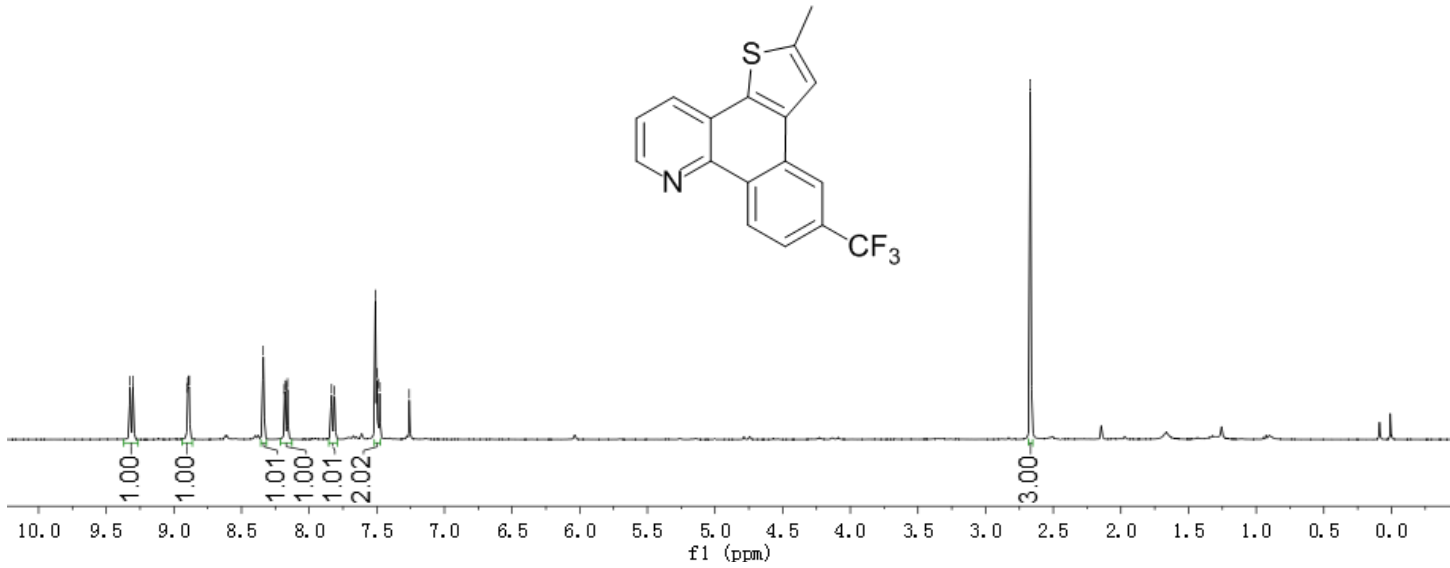

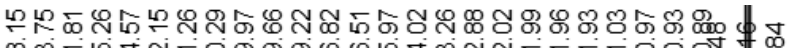

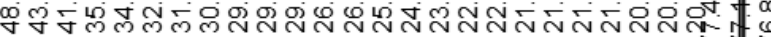
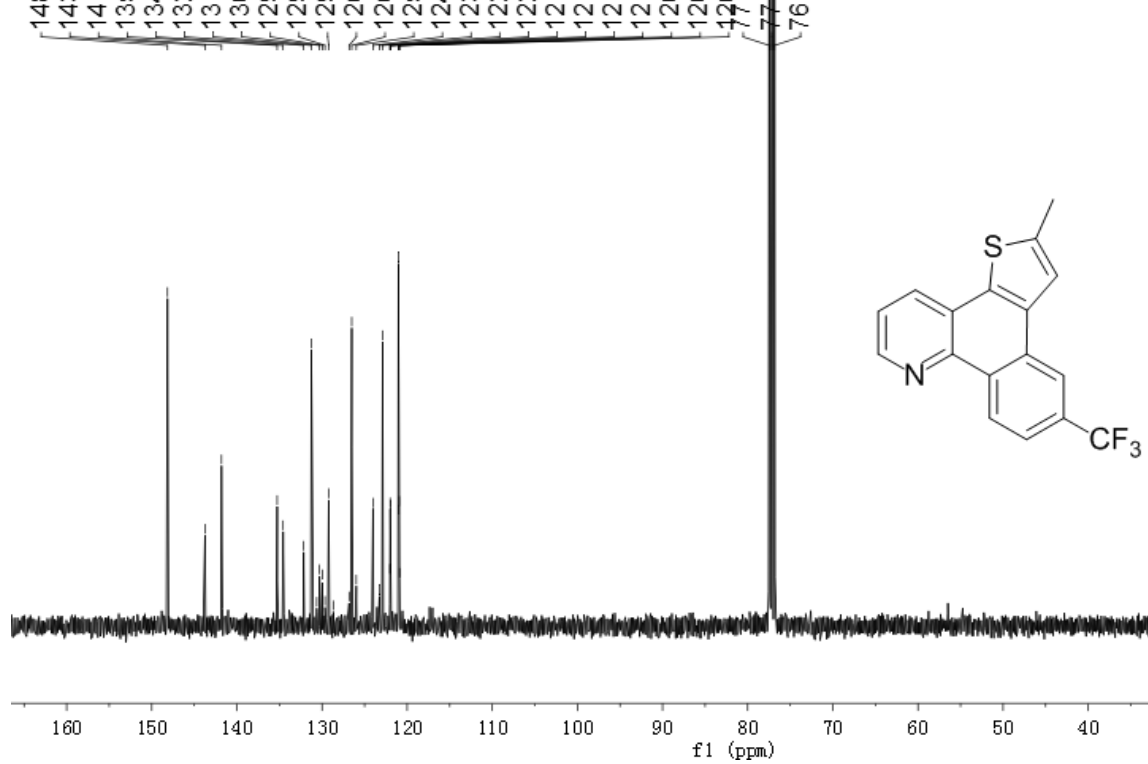
(2m)

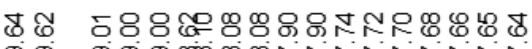

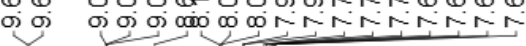

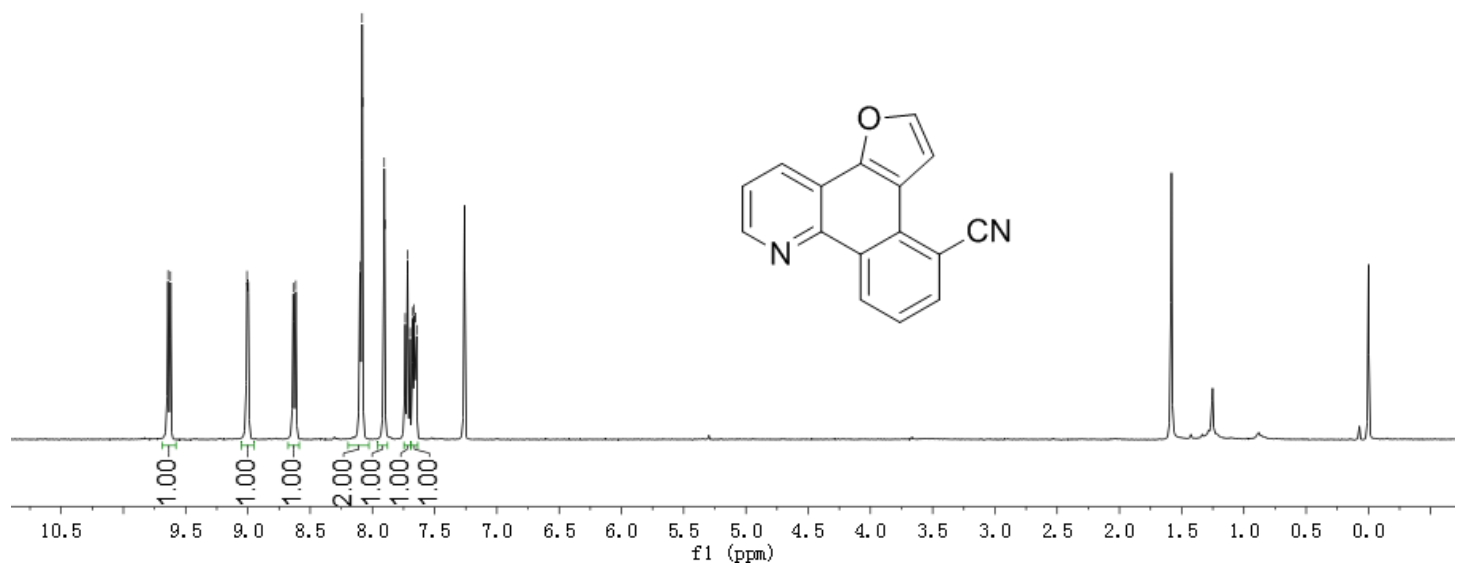

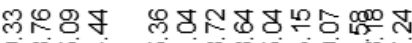

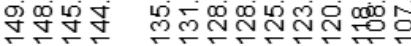
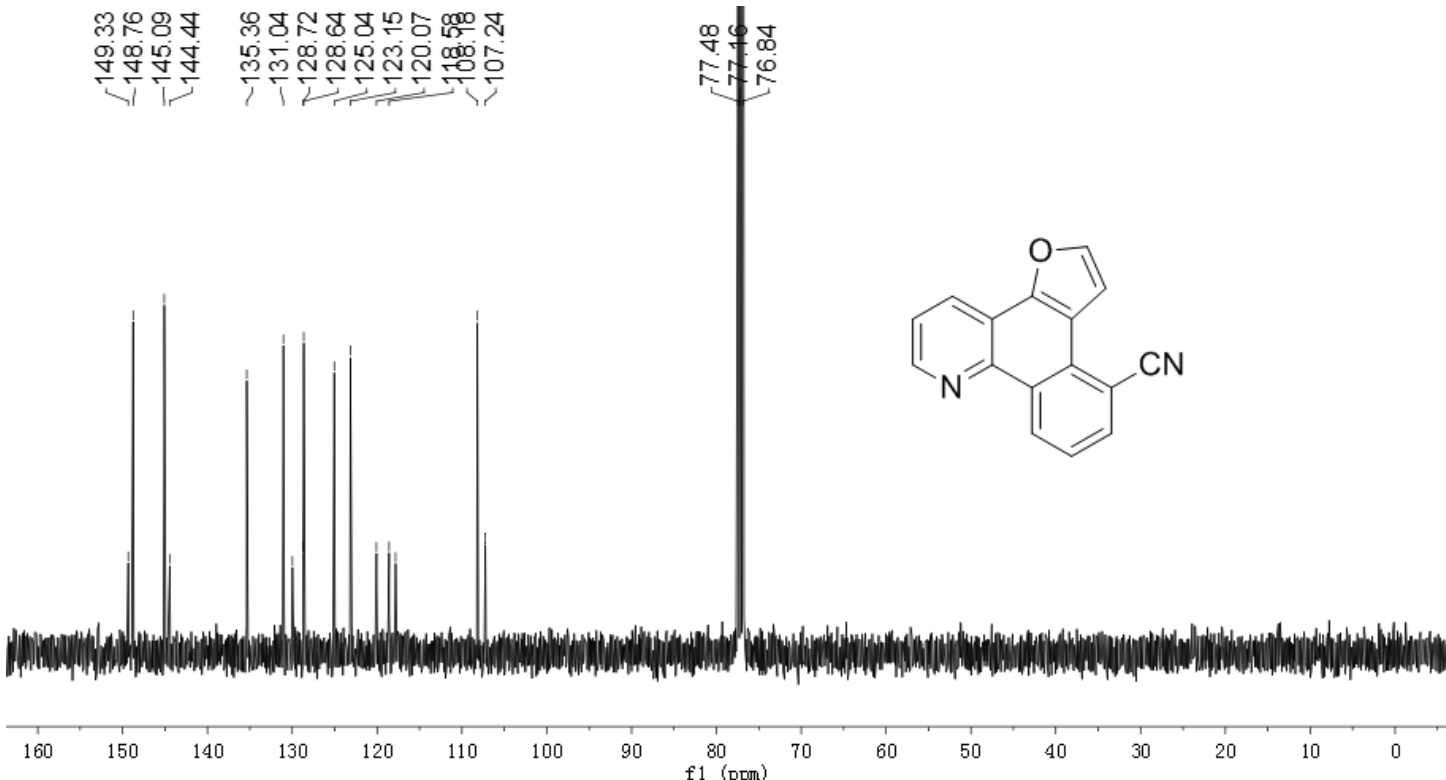


\section{(2n)}
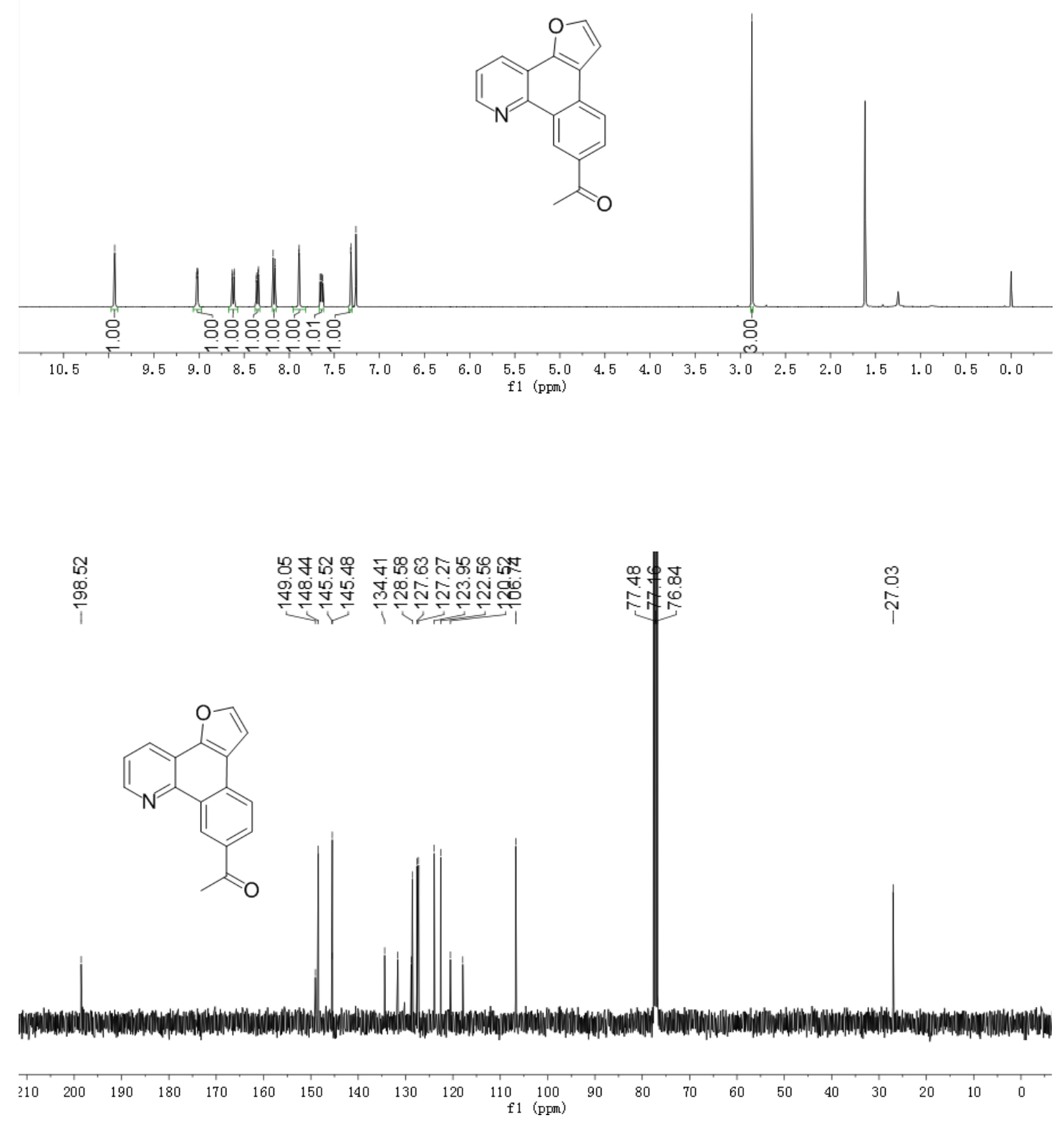


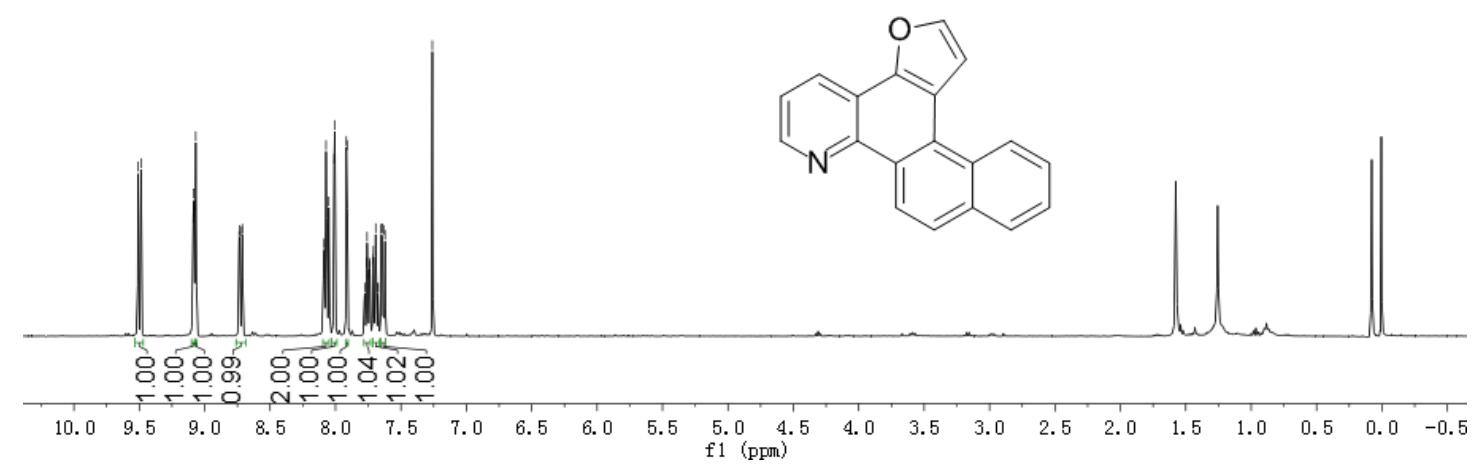

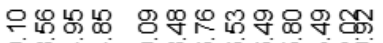

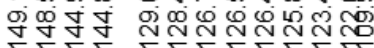

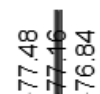

$-5-5$
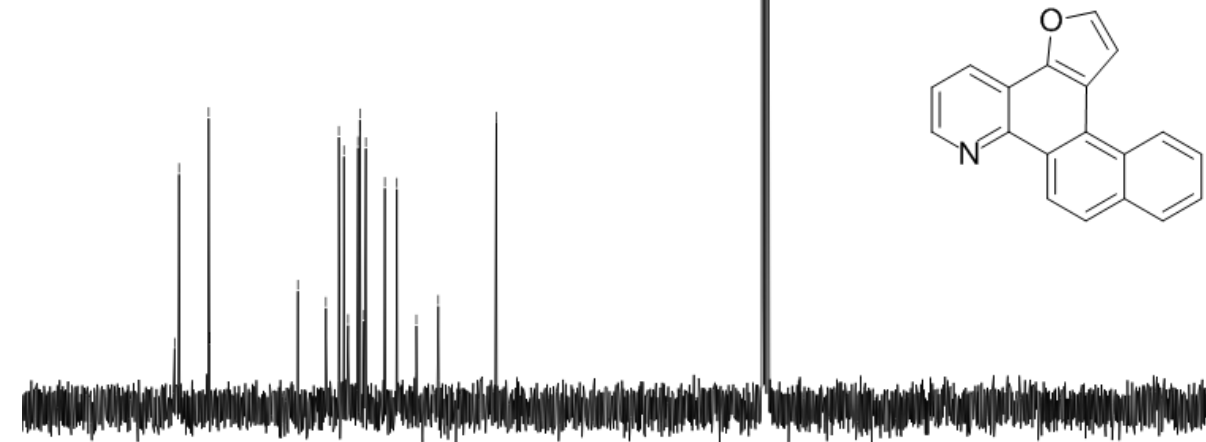

$160 \quad 150$

$140 \quad 130$

$120 \quad 110$

90

f1 (ppm) 
(2p)

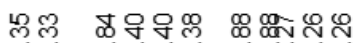

बij

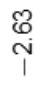
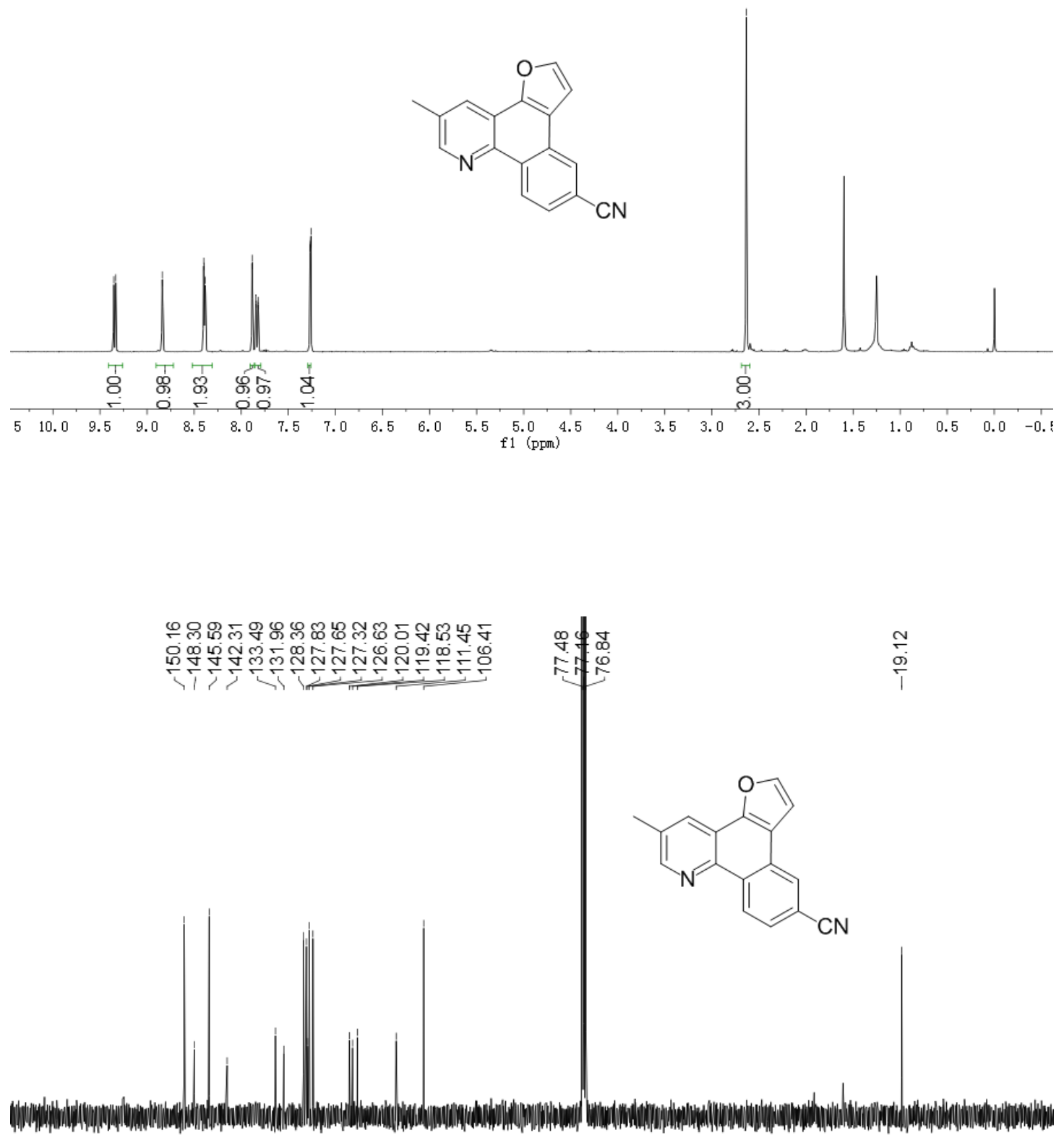

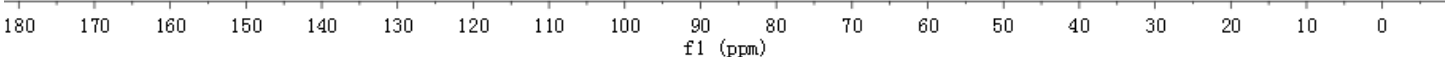


(2q)

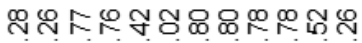

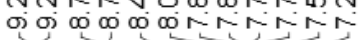

Non

กร
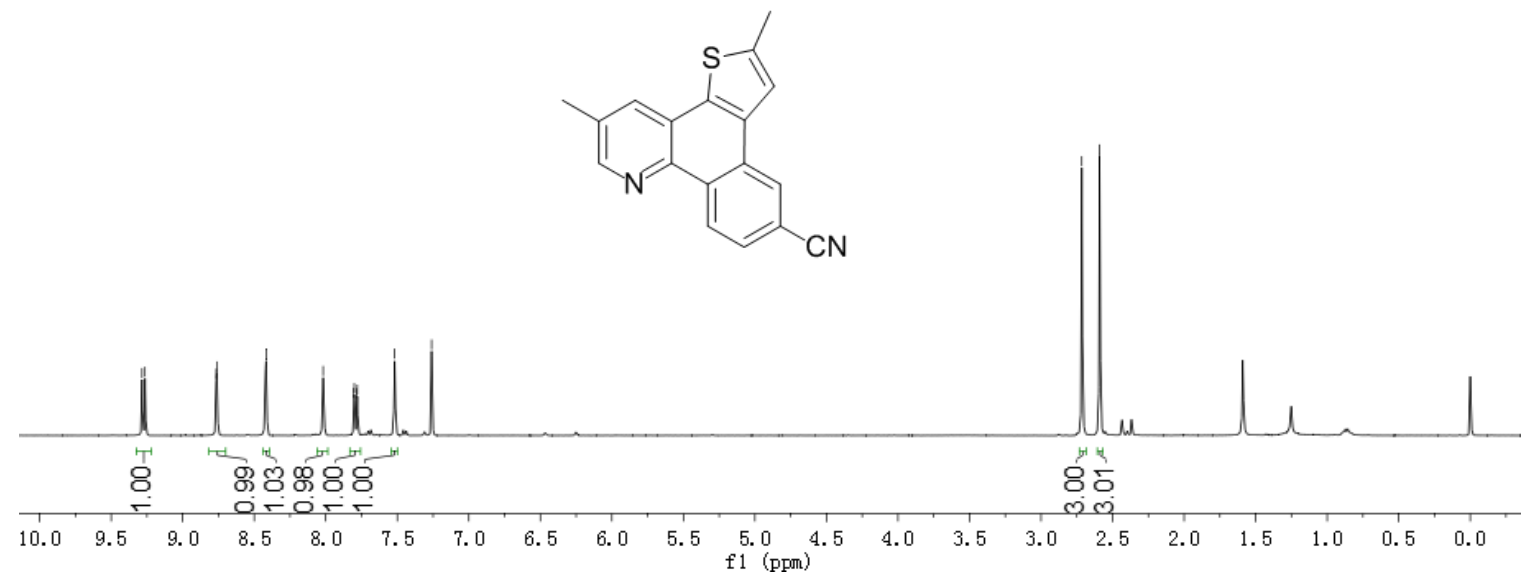

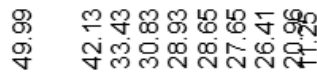

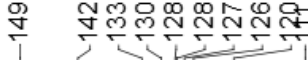

$\stackrel{\infty}{\infty}$

i)

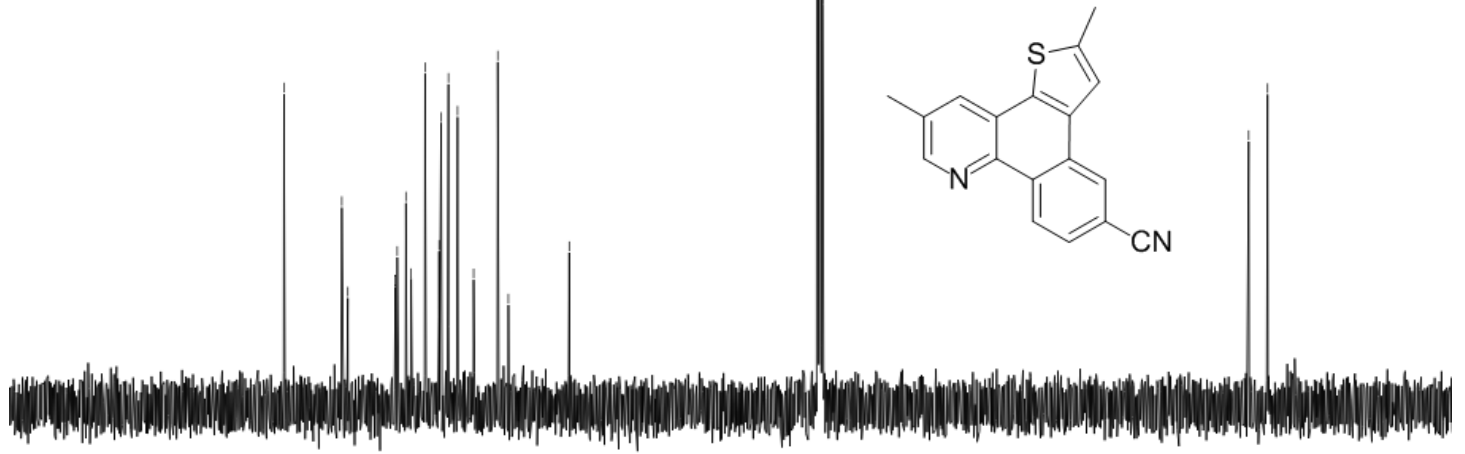

$100 \underset{f 1(\mathrm{DLm})}{90} 80$

$\begin{array}{llllllll}70 & 60 & 50 & 40 & 30 & 20 & 10 & 0\end{array}$ 
(2r)

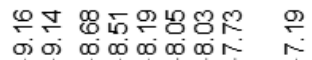

ஜํำ

कां क्ष

ก

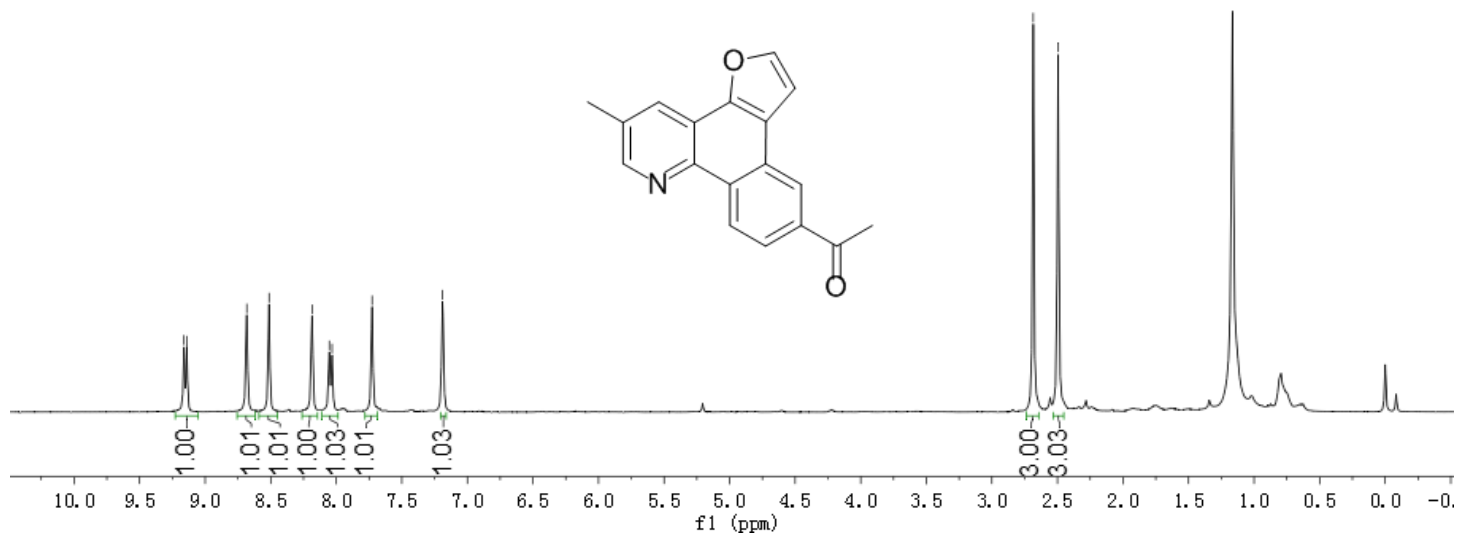

ભ্ল

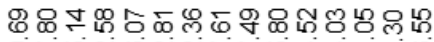

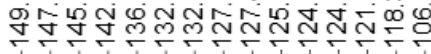

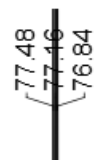

న⿻

Nำ<smiles>CC(=O)c1ccc2c(c1)c1ccoc1c1cc(C)cnc21</smiles>

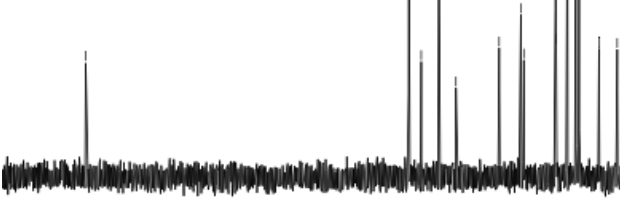
$\begin{array}{lllllllllllll}10 & 200 & 190 & 180 & 170 & 160 & 150 & 140 & 130 & 120 & 110 & 100 & 90 \\ \mathrm{f} 1 & (\mathrm{ppm})\end{array}$ 
(2s)

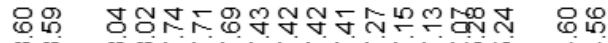

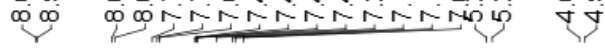

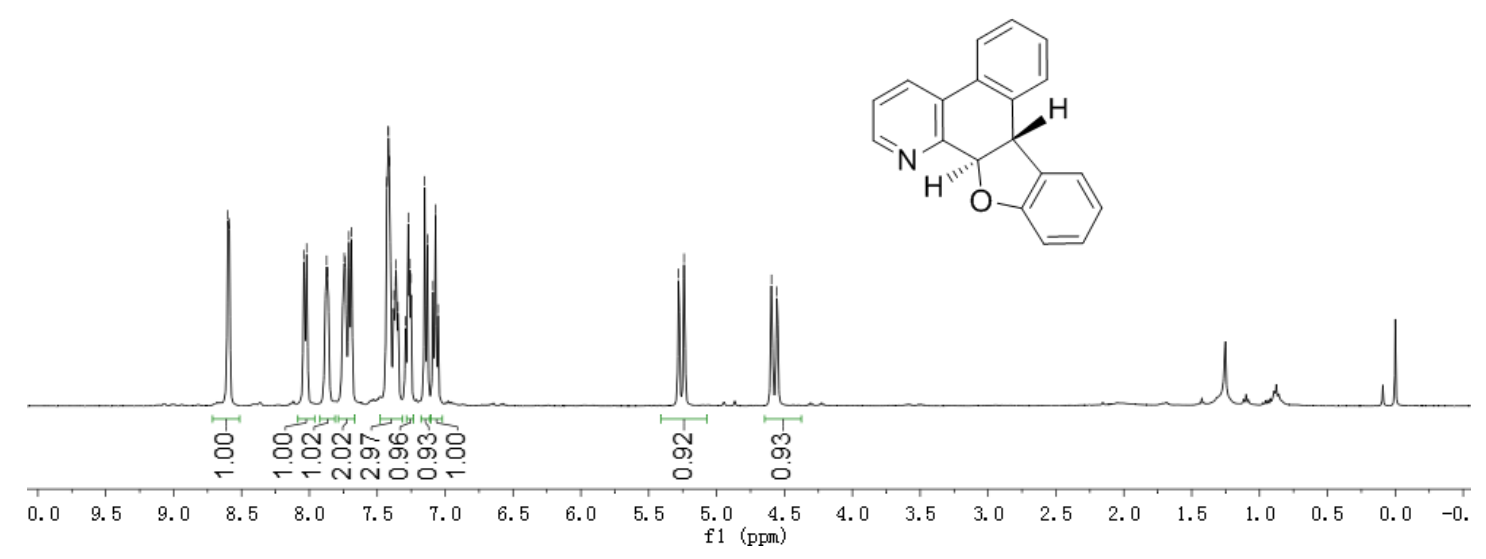

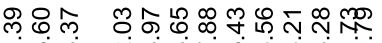

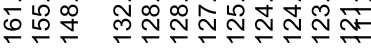

万.

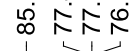

$\stackrel{\substack{\infty \\ \infty}}{\stackrel{\infty}{+}}$

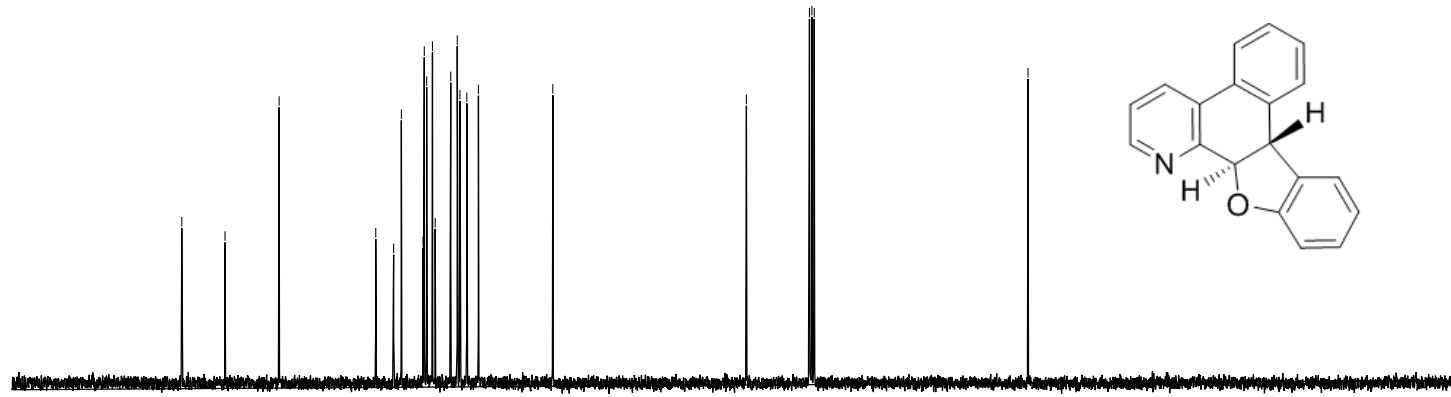

$\begin{array}{lllllllllllllllllll}180 & 170 & 160 & 150 & 140 & 130 & 120 & 110 & 100 & 90 & 80 & 70 & 60 & 50 & 40 & 30 & 20 & 10 & 0\end{array}$ 
舟
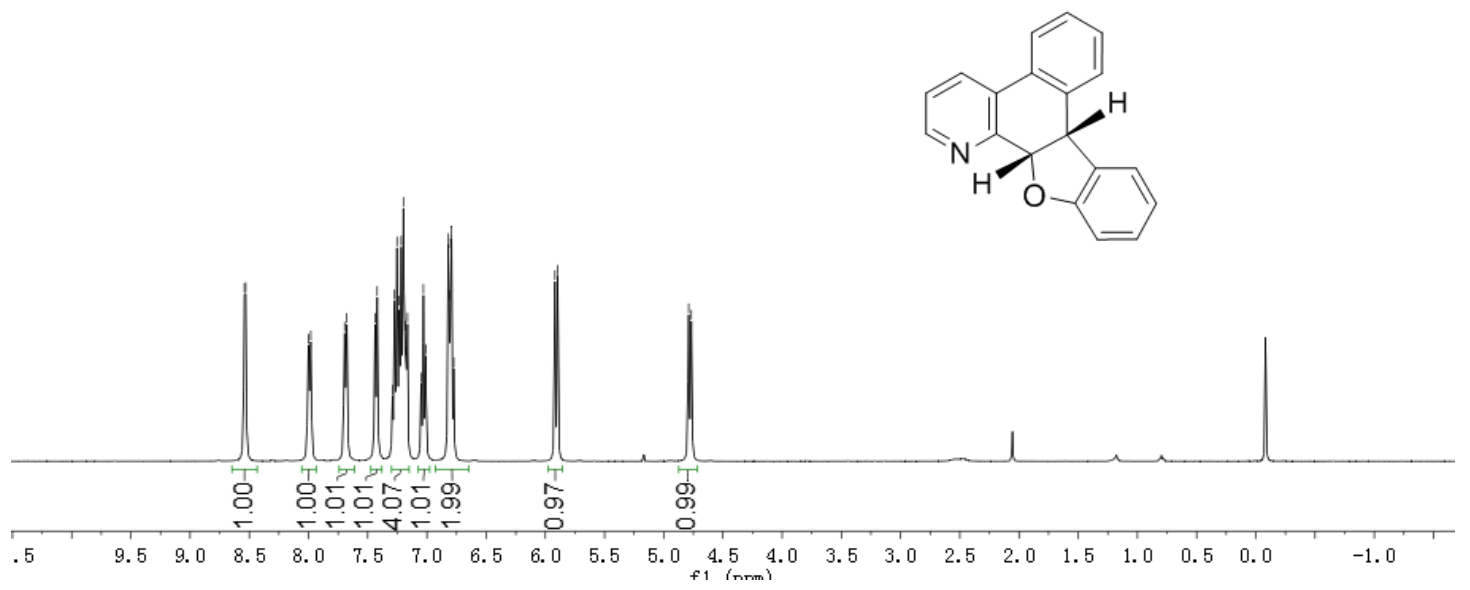

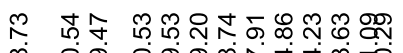

吕 윰

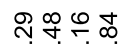

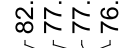

id
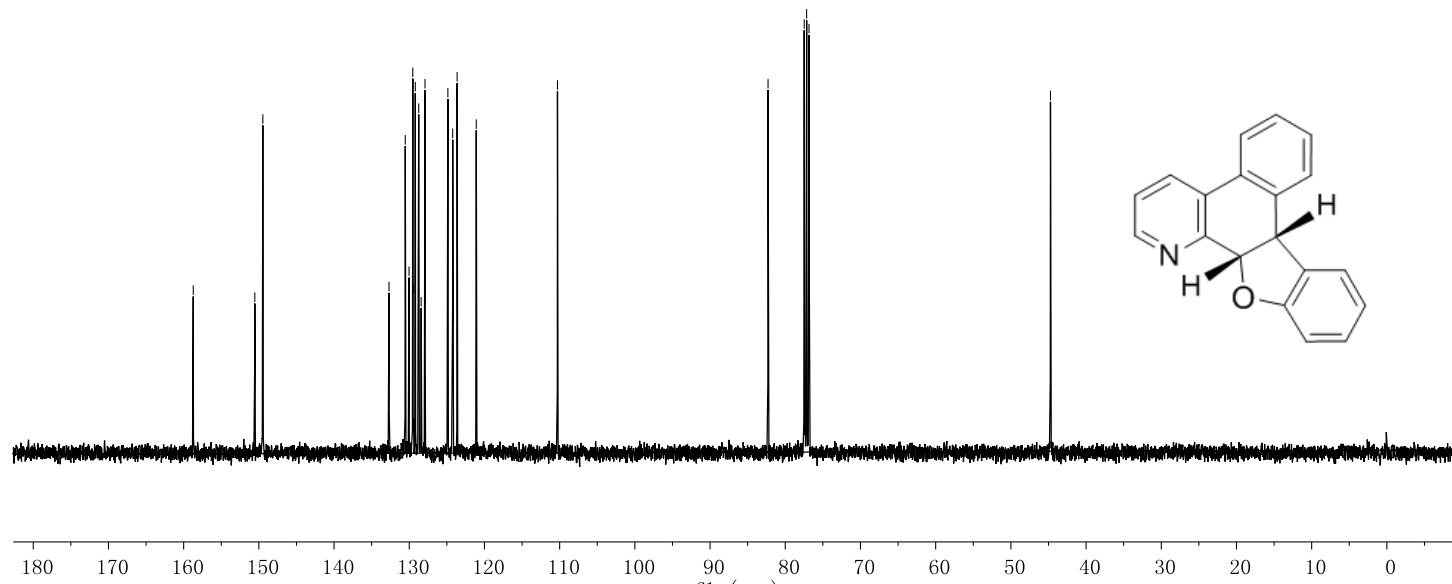

90
$\mathrm{f} 1(\mathrm{ppm})$ 
(2u)

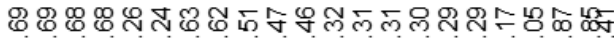
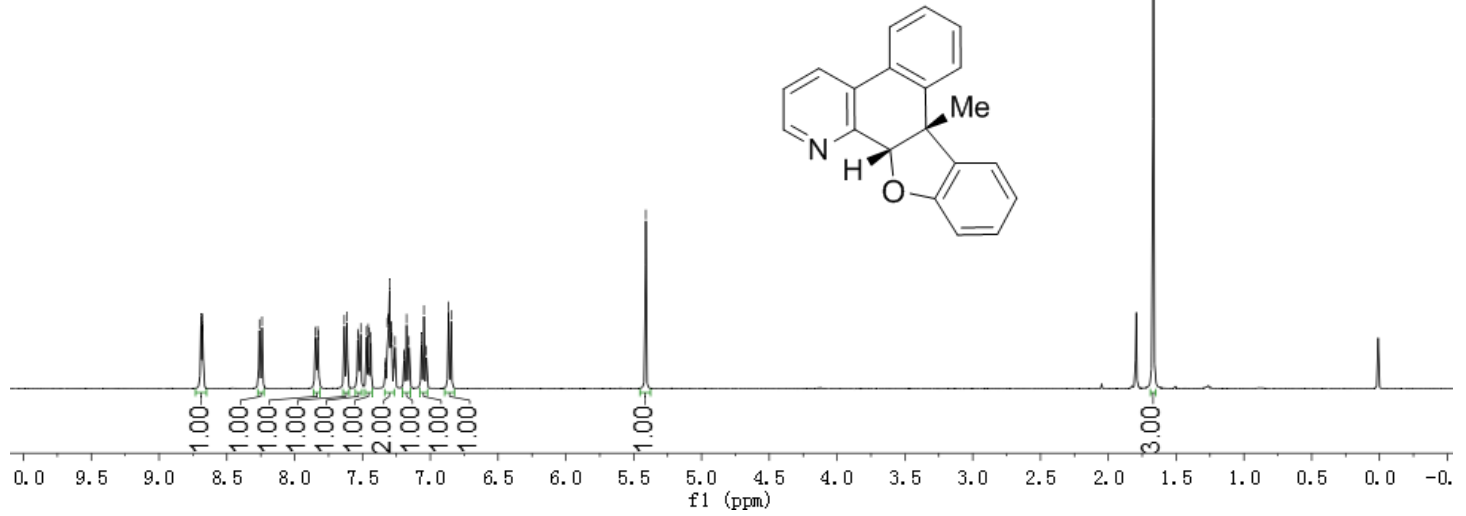

i

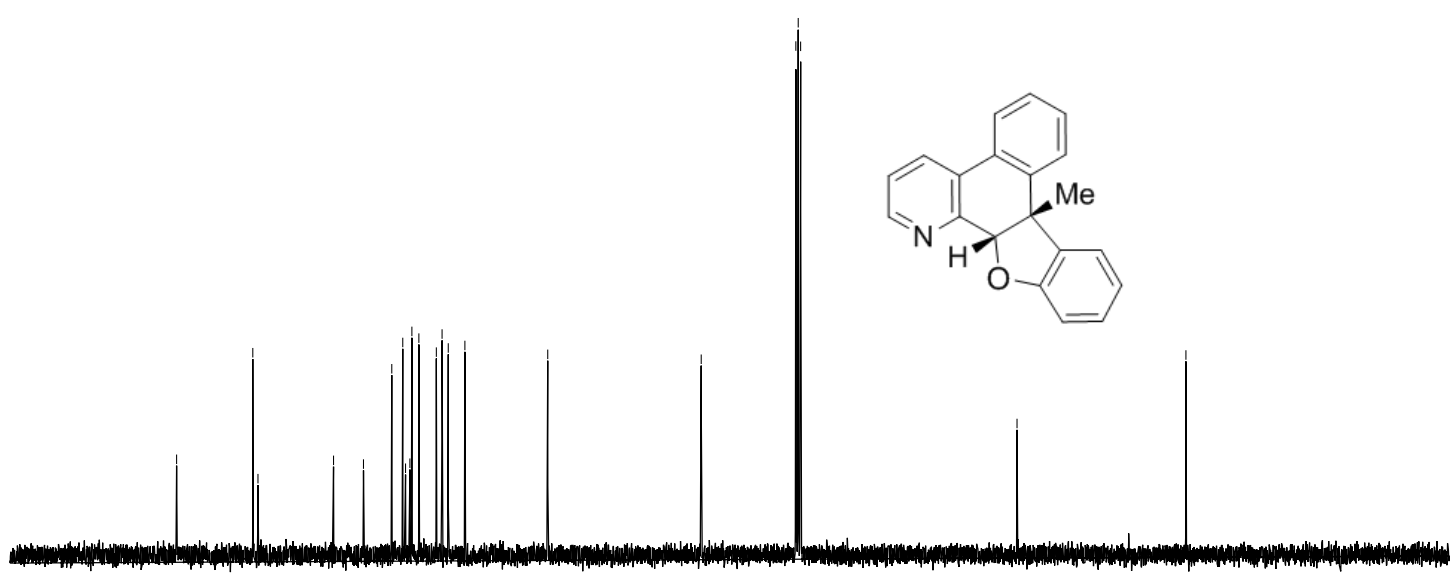

$170 \quad 160 \quad 150$

f1 (ppm) 
$(2 \mathbf{v})$

뚜음ำ

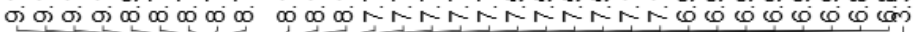

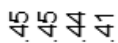

ninin
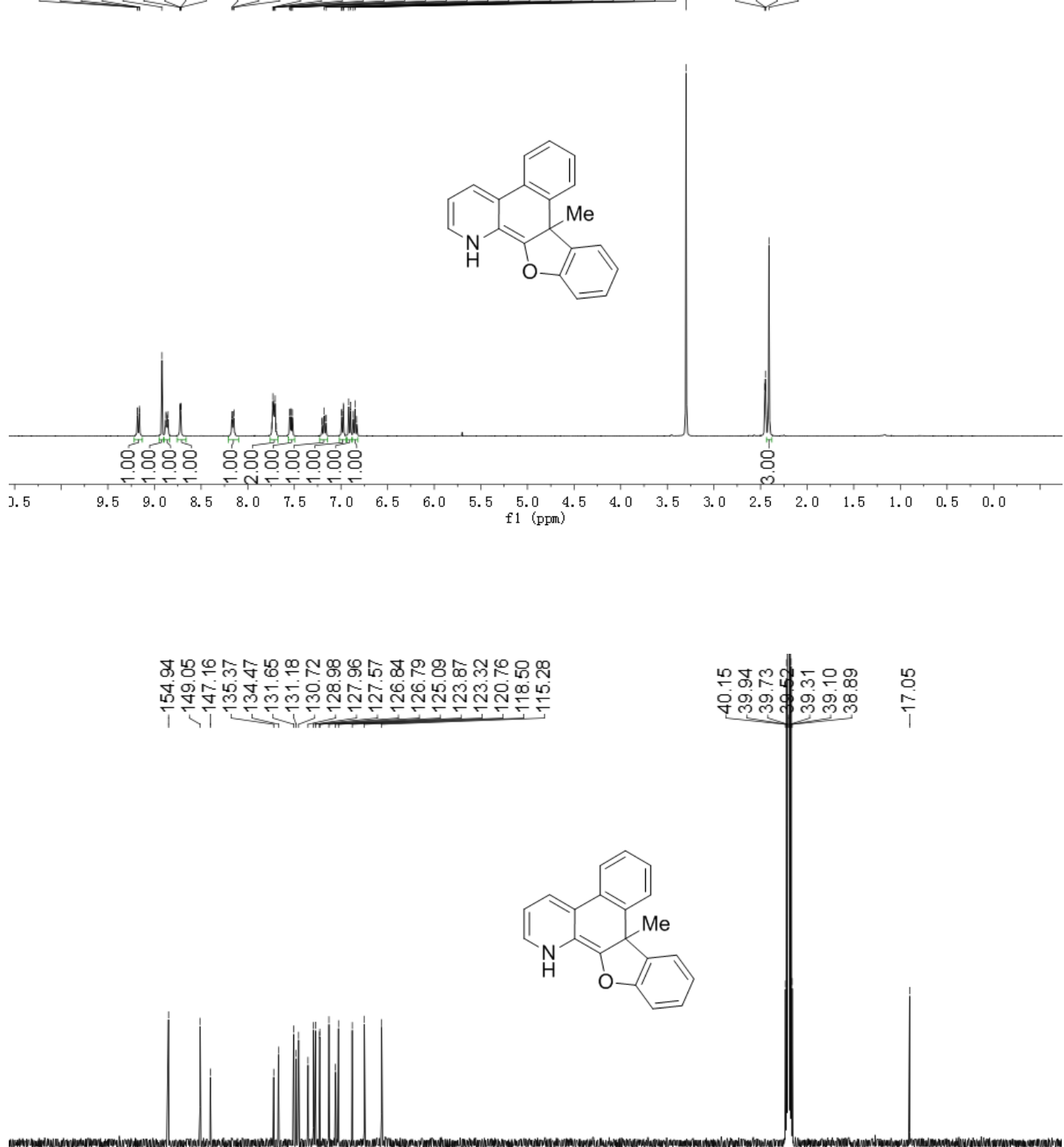

$\begin{array}{llllllllll}180 & 170 & 160 & 150 & 140 & 130 & 120 & 110 & 100 & 90 \\ \mathrm{f} 1(\mathrm{ppm}) & 80\end{array}$ 


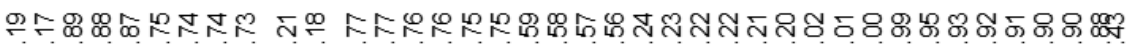

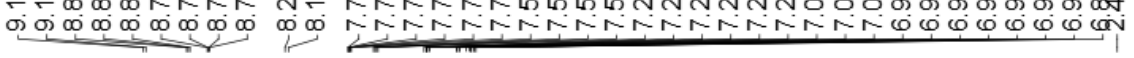

${ }^{1} \mathrm{H}$ NMR $\left(400 \mathrm{MHz}, \mathrm{DMSO}+\mathrm{D}_{2} \mathrm{O}\right)$

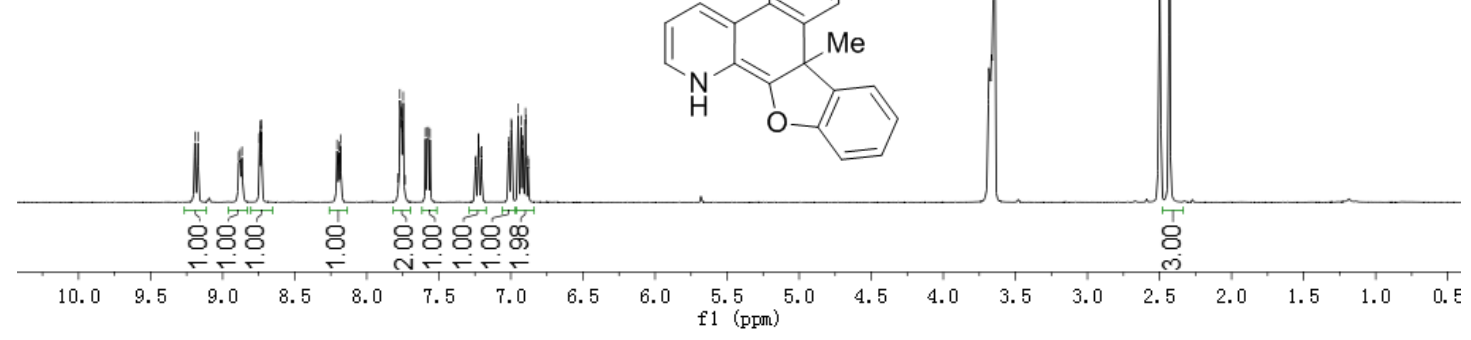

(5a)

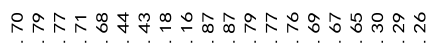

a

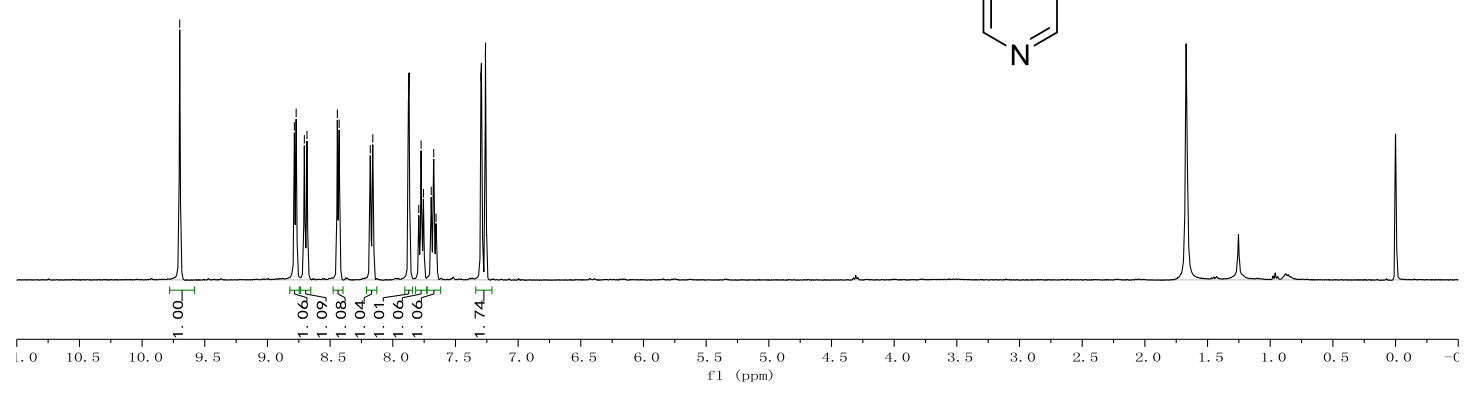

113 


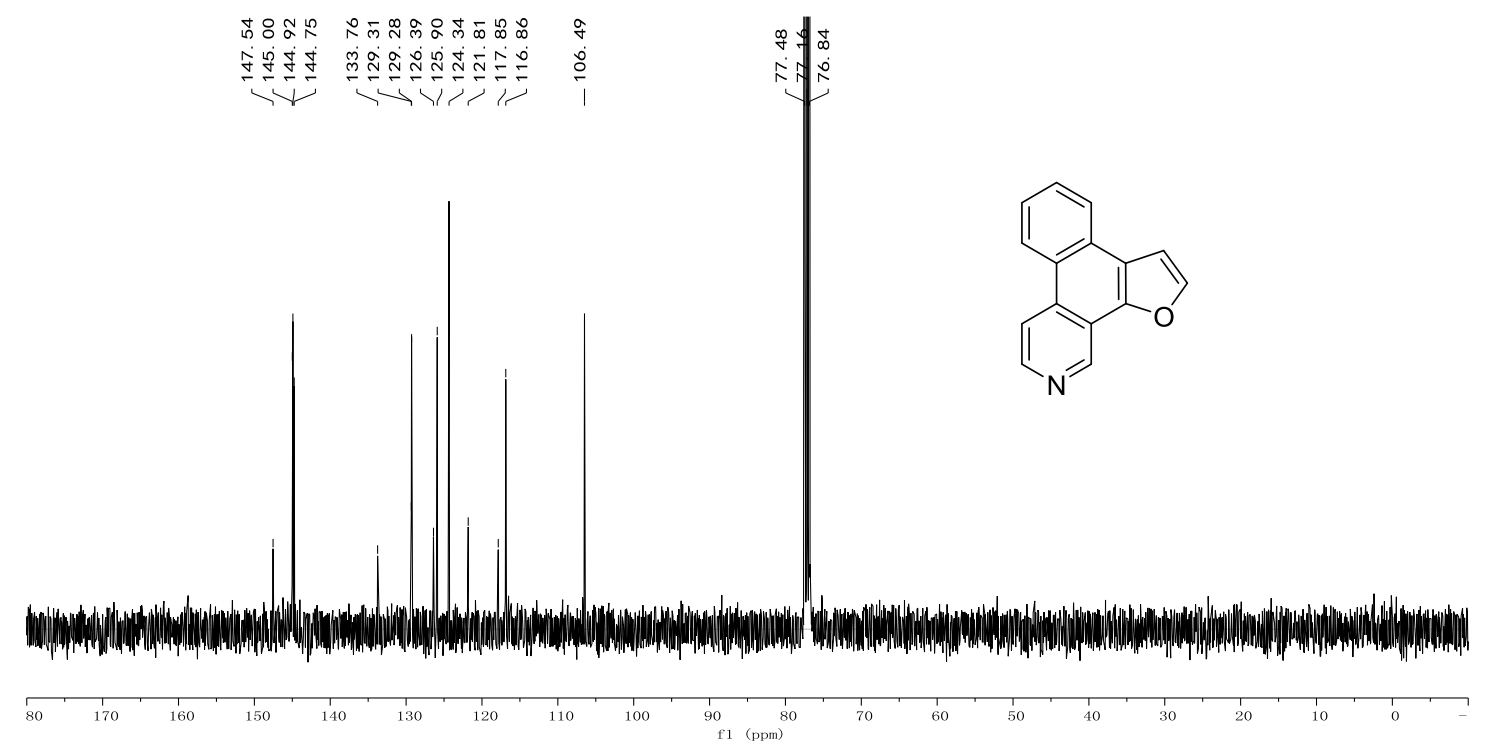

(5b)

๓

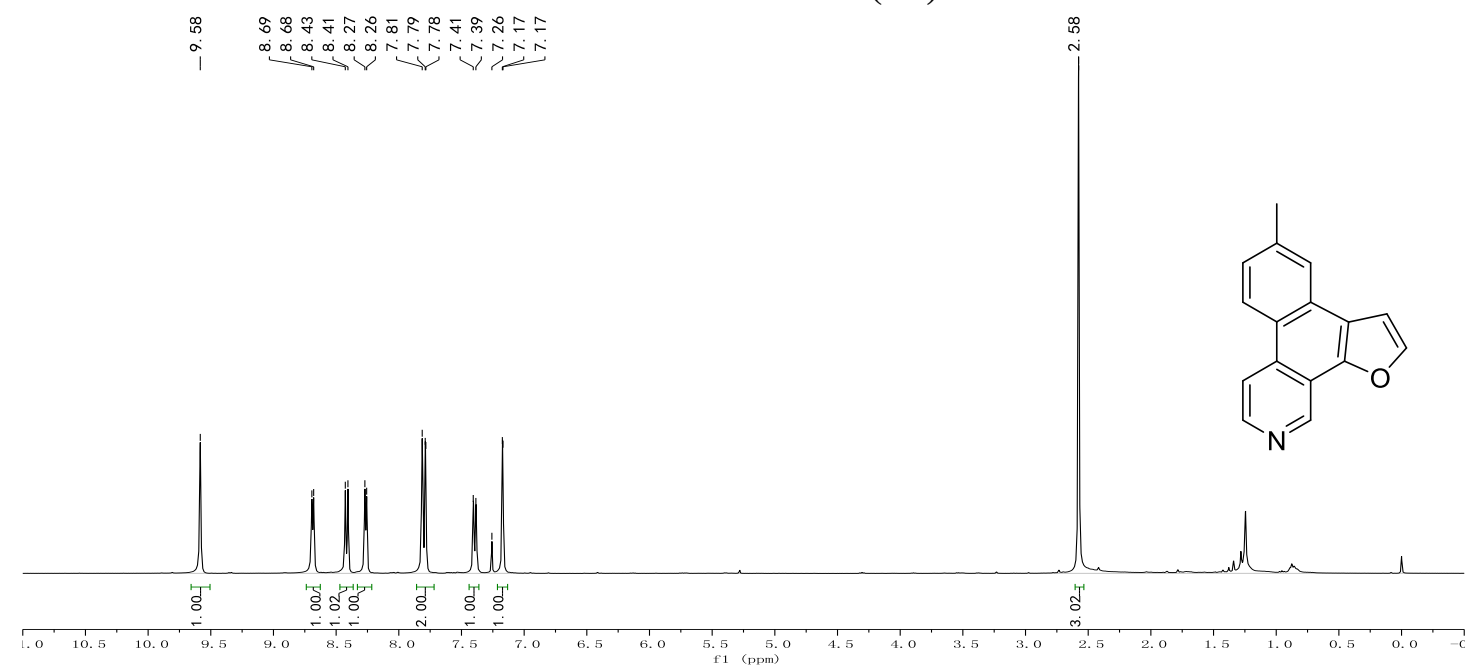




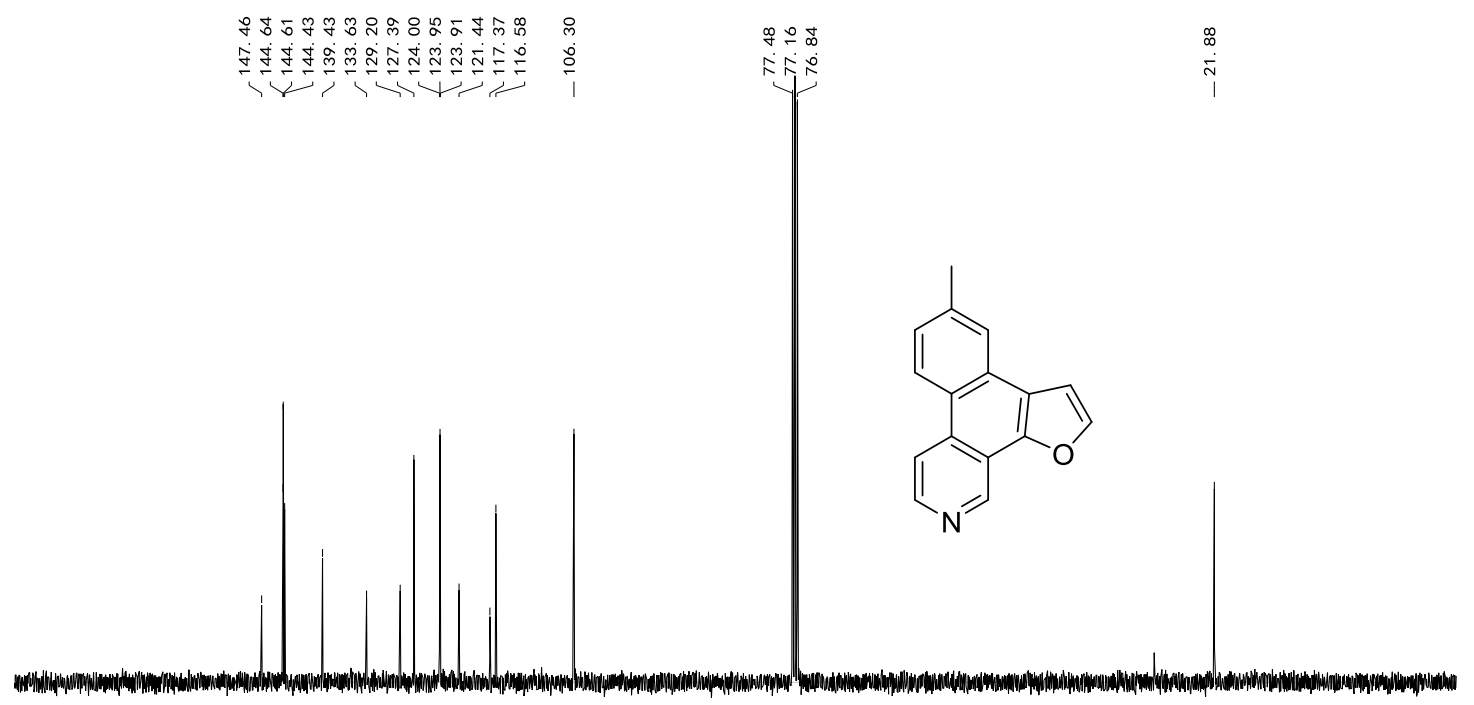

(5c)

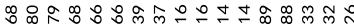

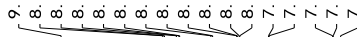

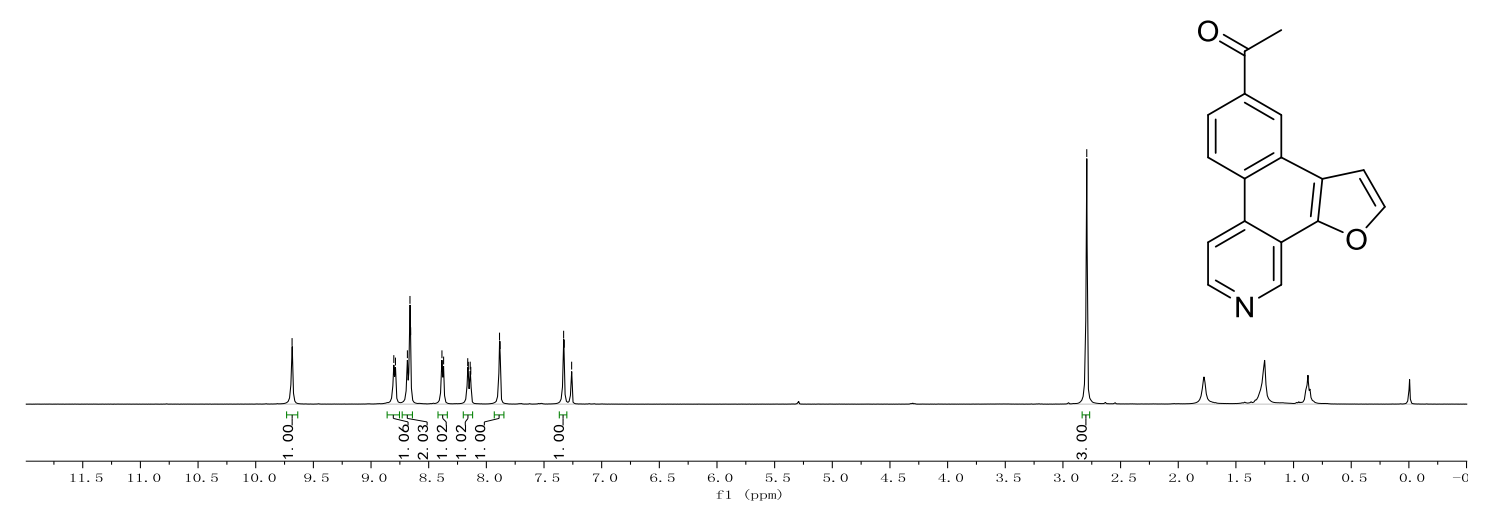



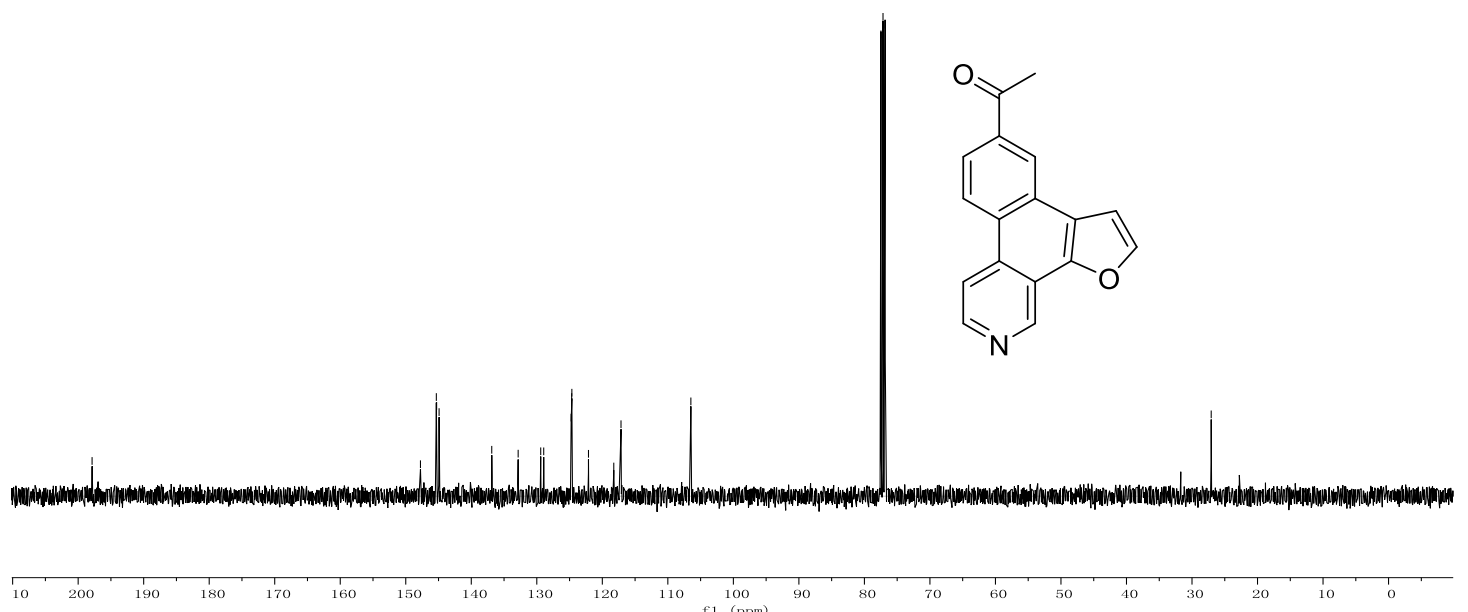

(5d)

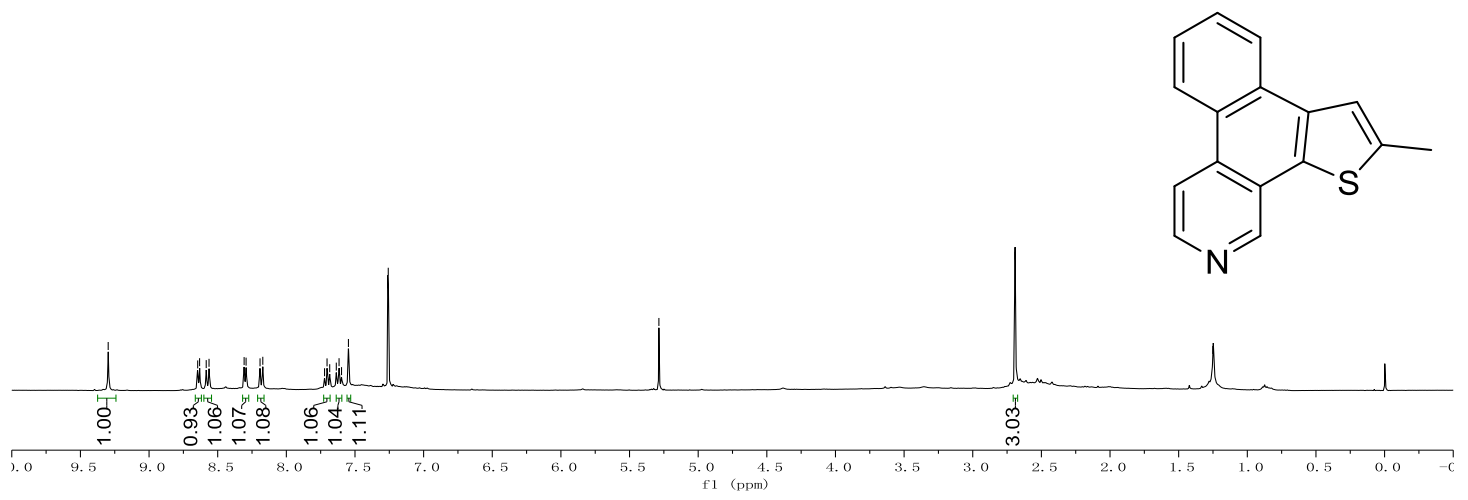




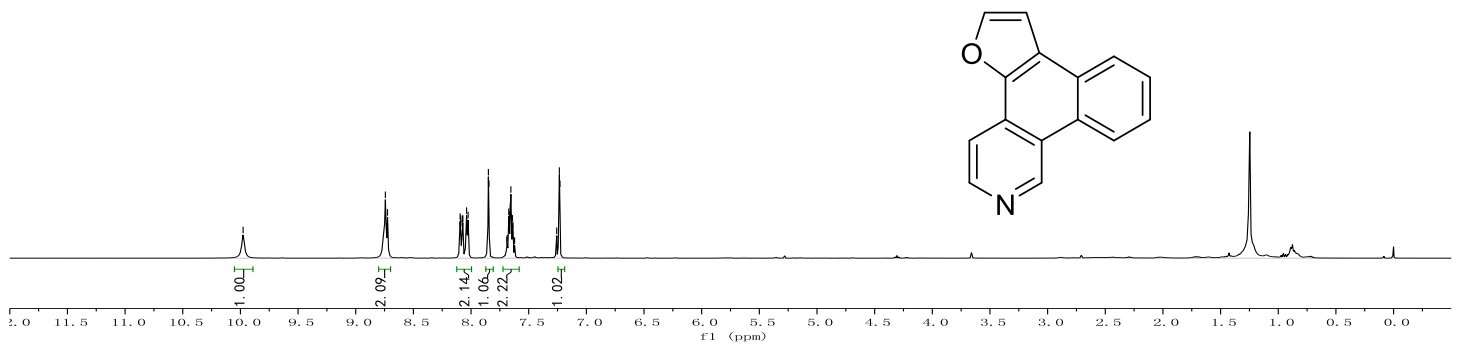

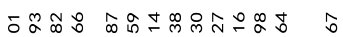

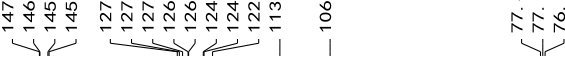

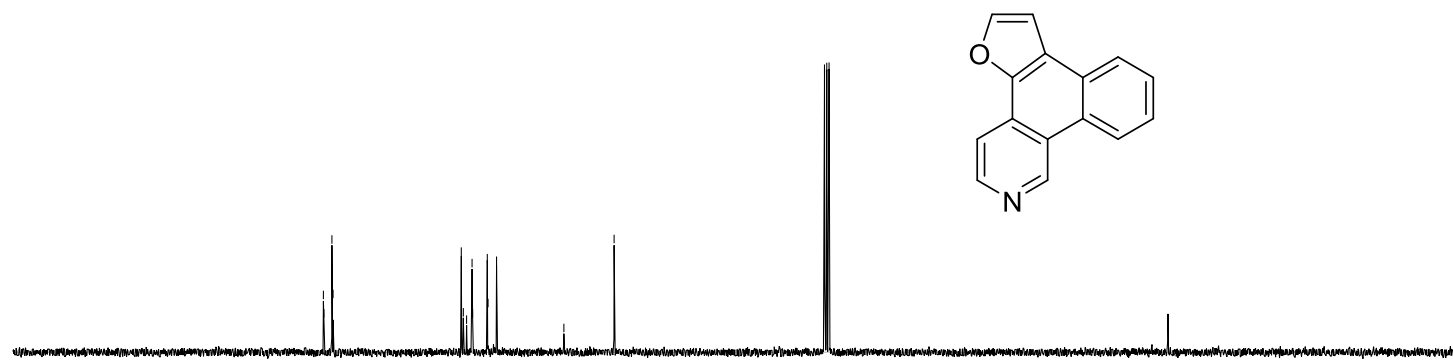

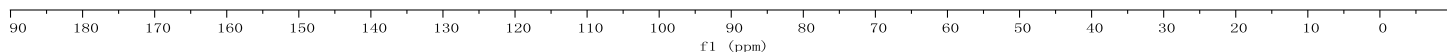


(6b)

œ

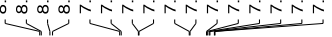

$\stackrel{\substack{\infty \\ \dot{m} \\ \dot{m}}}{1}$

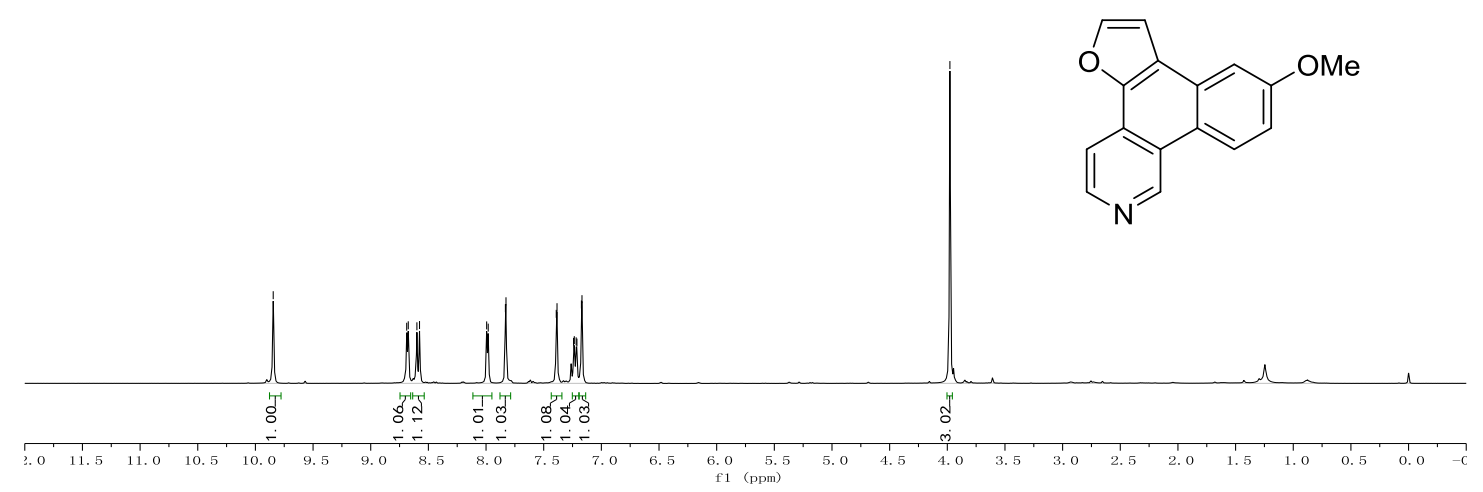

\begin{tabular}{|c|c|c|}
\hline 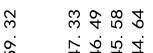 & 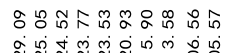 & $\stackrel{\infty}{\stackrel{0}{\longleftarrow}}$ \\
\hline$\underbrace{-\sqrt[1]{2}}$ & 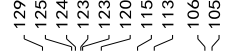 & 兵易 \\
\hline
\end{tabular}

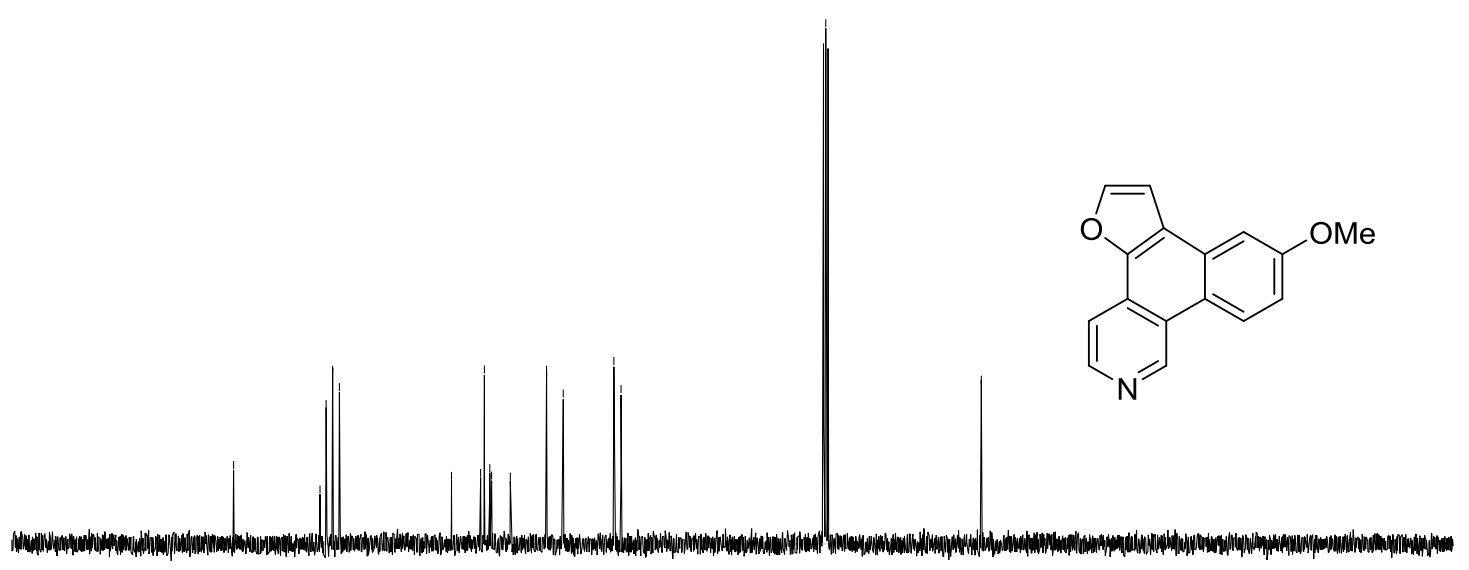

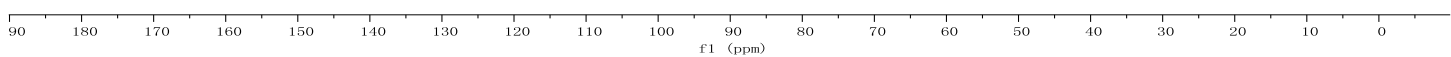


(6c)

a)

$\underbrace{\infty} \underbrace{\infty} \underbrace{\infty} \infty \underbrace{\infty}$

$\stackrel{i}{i}$

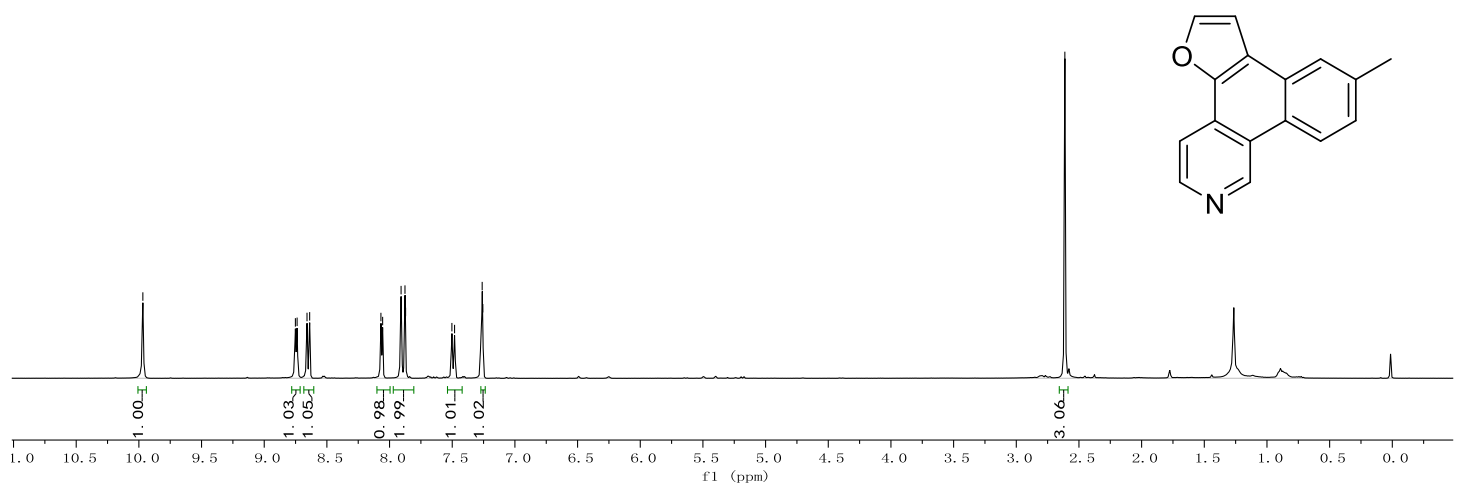

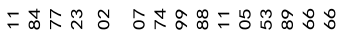

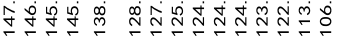

学赵

i.j.

岤

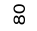

$\dot{\sim}$

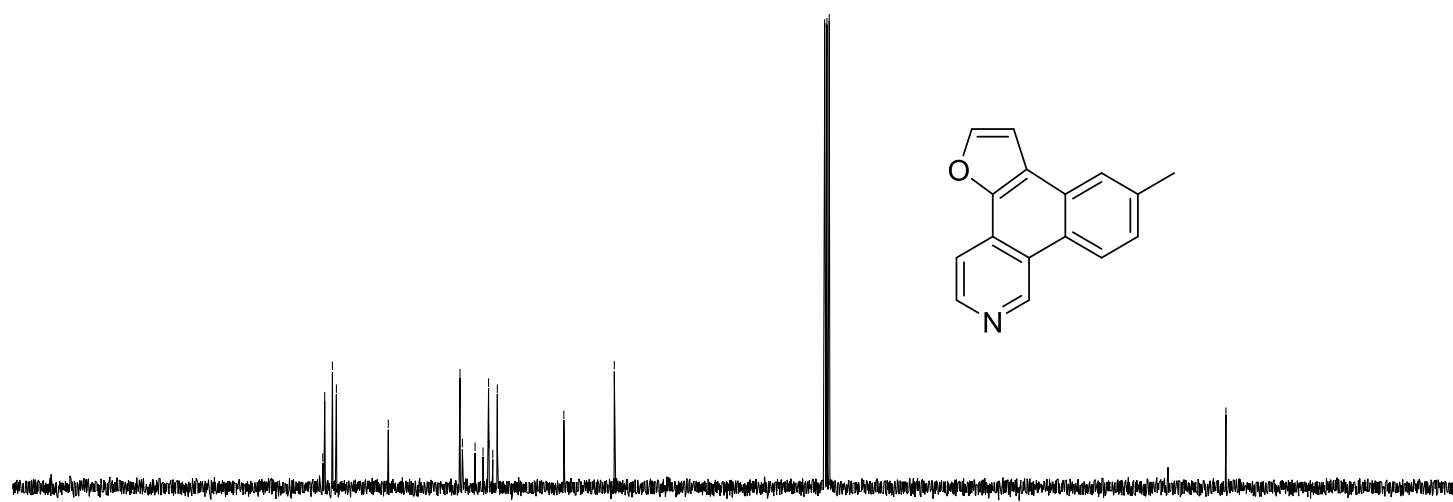

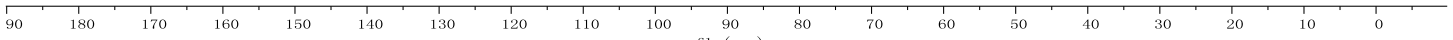


(6d)

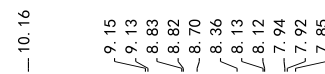

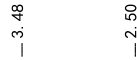

aldili

"so

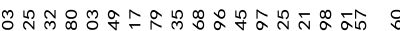

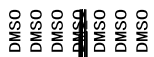

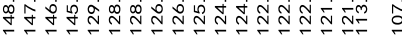

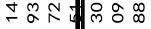

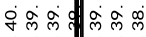
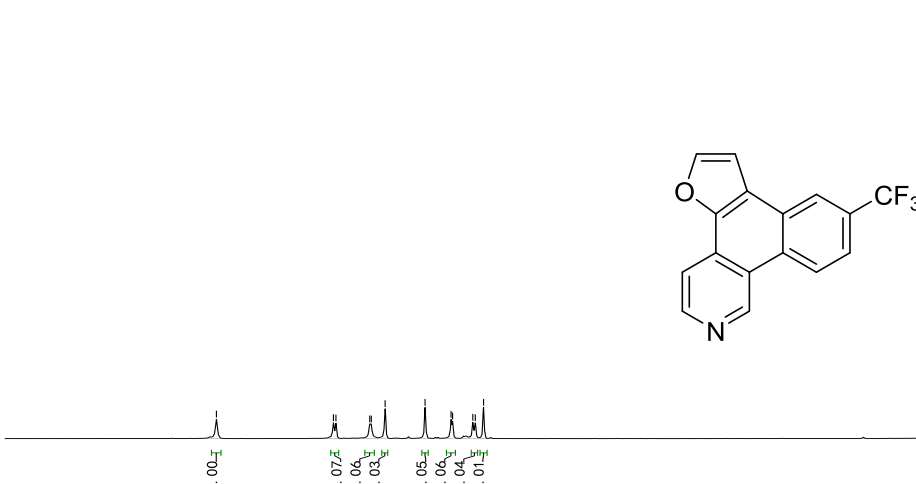

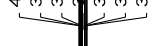

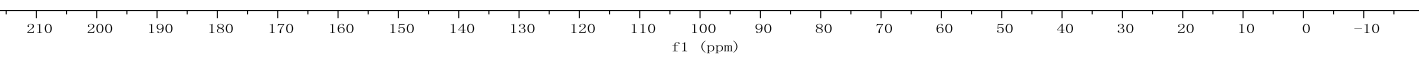


(6e)

กั

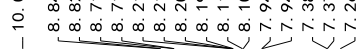

$\stackrel{\substack{\infty \\ \stackrel{\infty}{1} \\ i}}{2}$

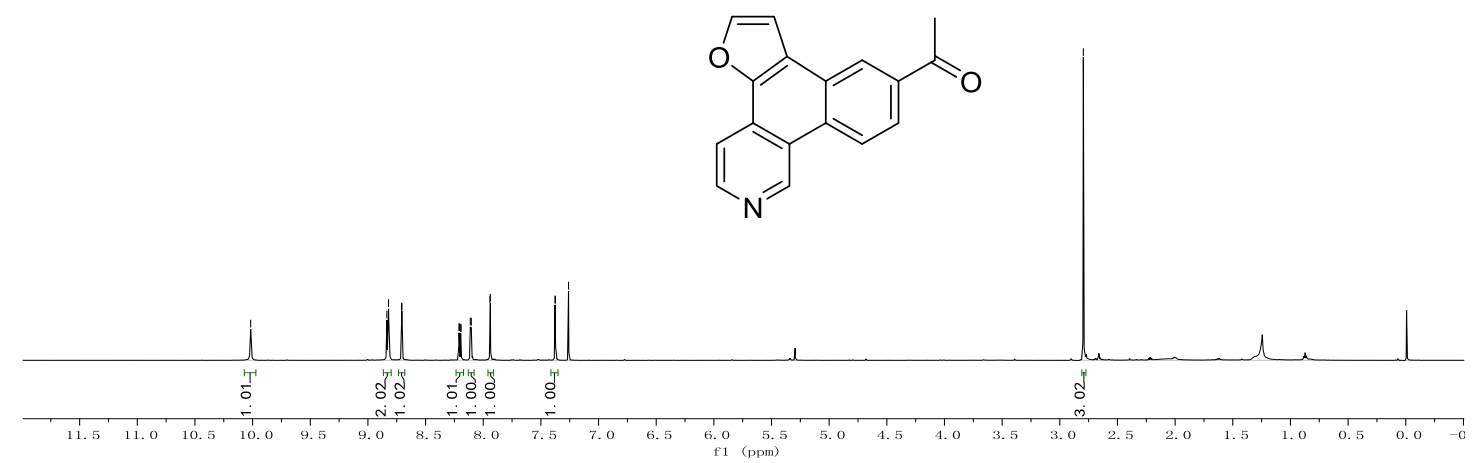

$\stackrel{+}{\infty}$

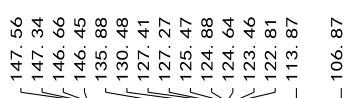

的捛

$\underset{\substack{0 \\ \stackrel{n}{i}}}{i}$<smiles>CC(=O)c1ccc2c3cnccc3c3occc3c2c1</smiles>

14.4.

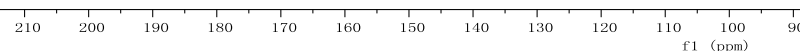


(6f)

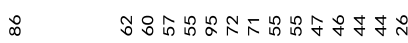

i $\quad \underbrace{\infty}_{\infty}$

용

i)

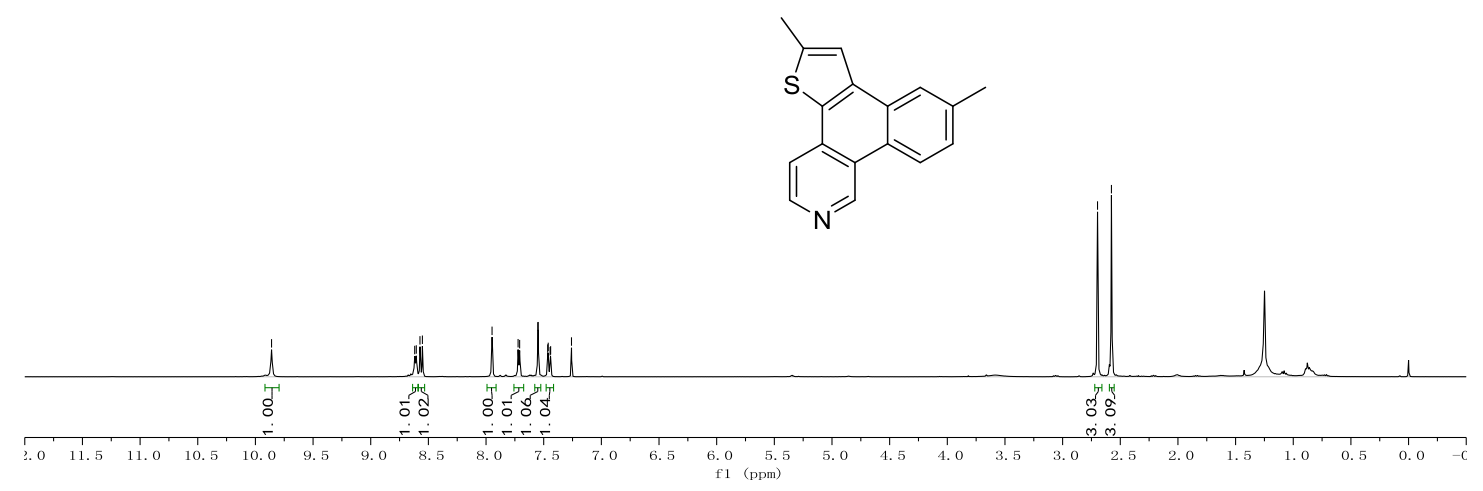

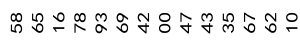

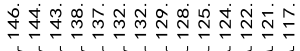

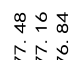

s f

촌

$\stackrel{i}{i} \stackrel{0}{i}$

पर प

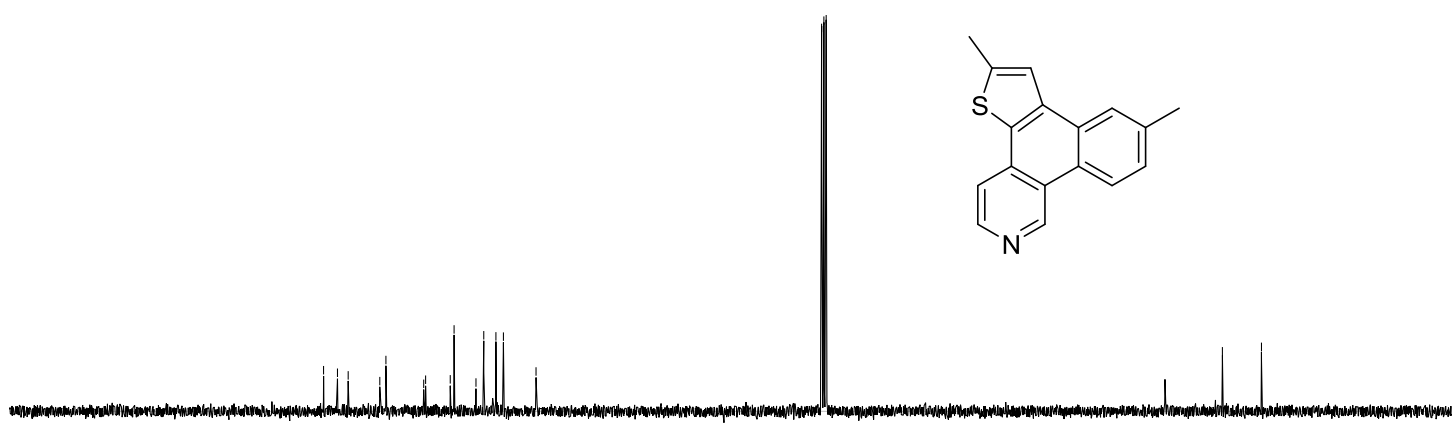

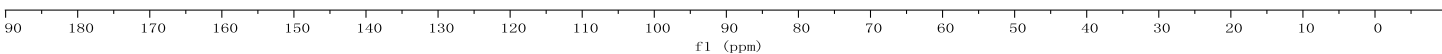


(6g)

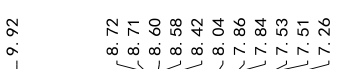

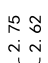

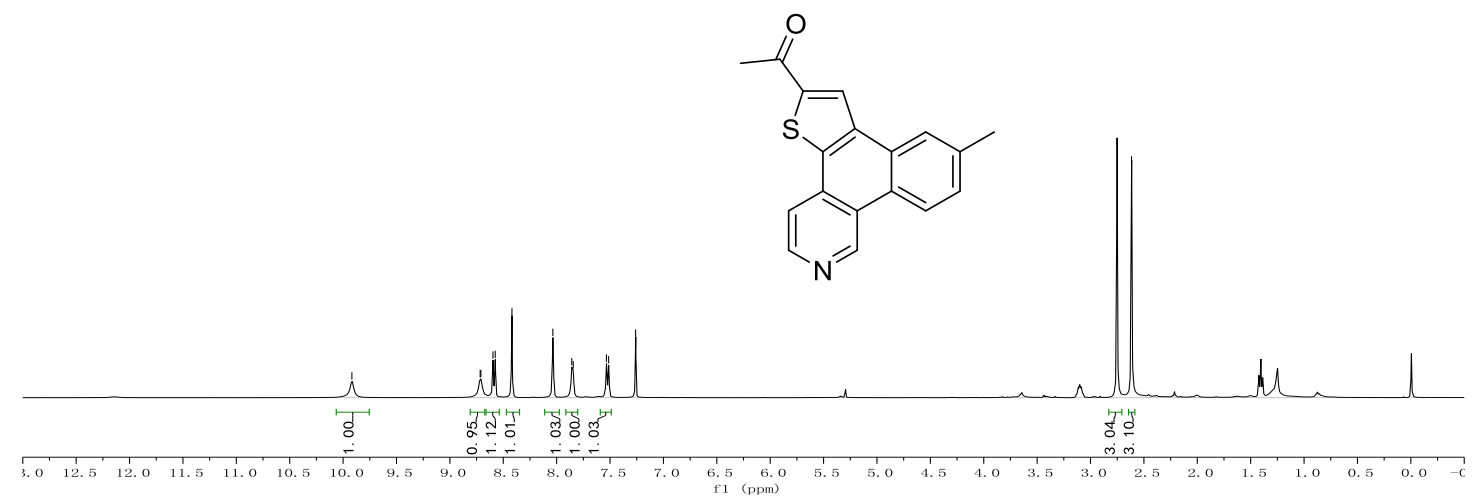

$\frac{a}{5}$

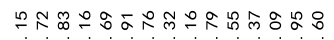

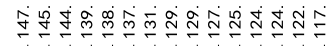

응

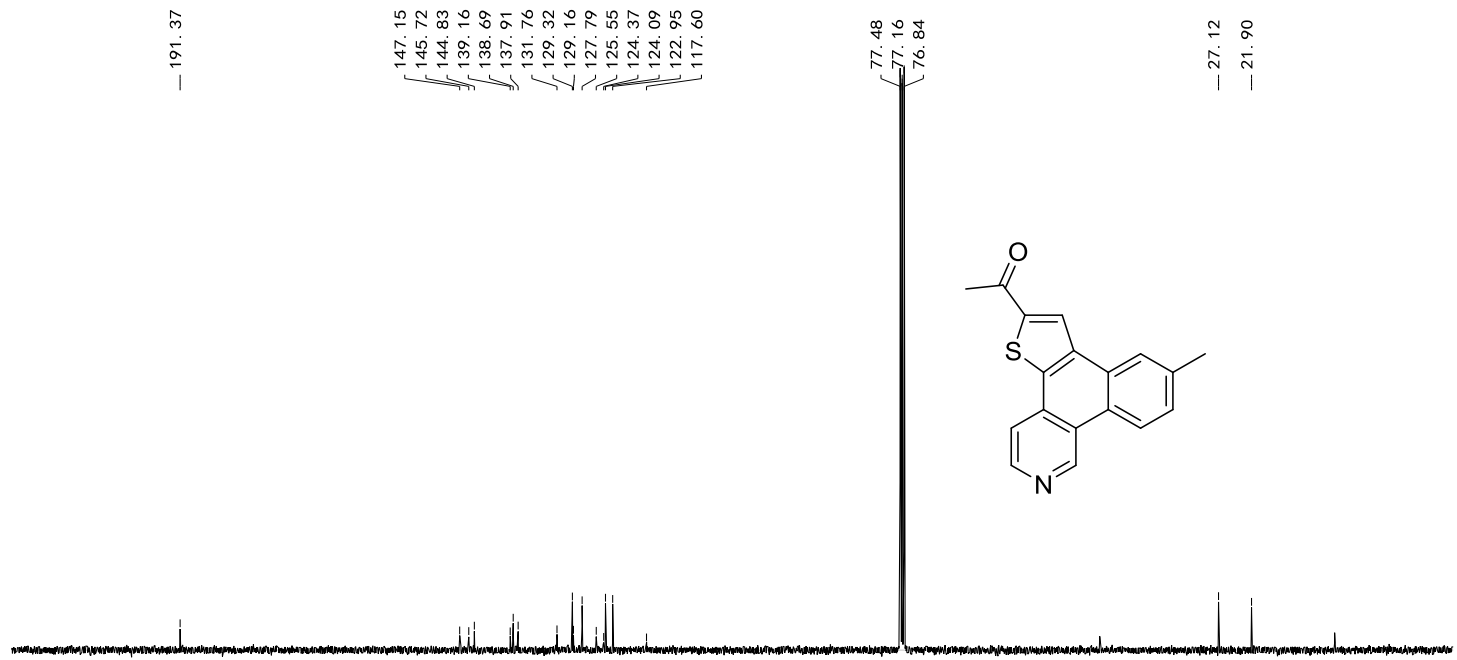


(6h)

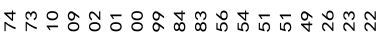

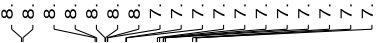

$\stackrel{m}{m}$

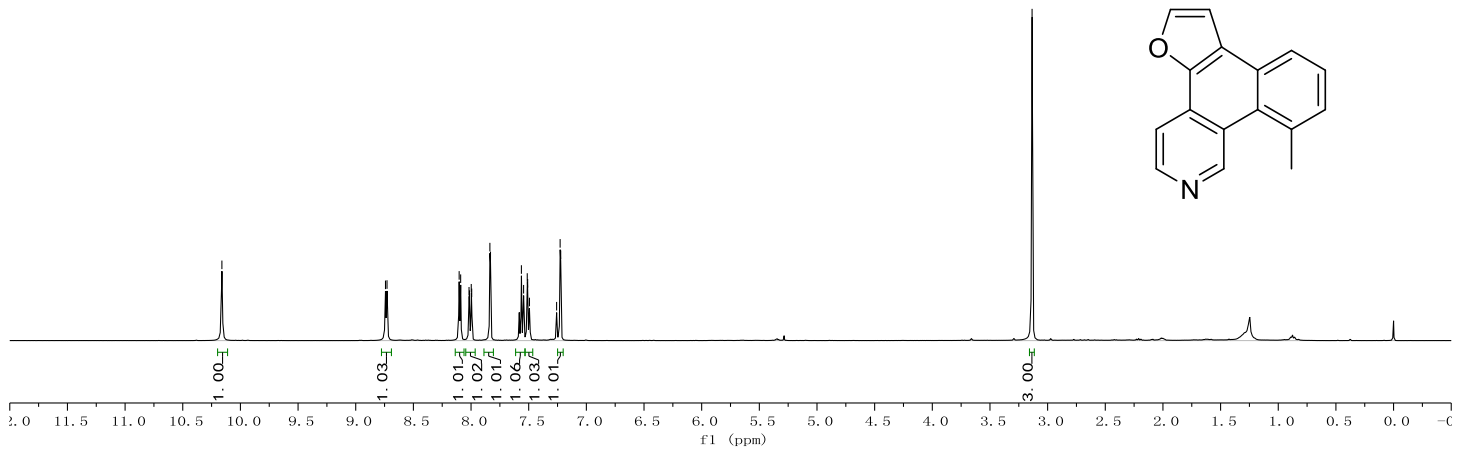

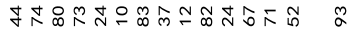

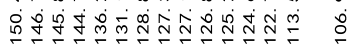

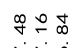

र下宓

$\stackrel{\infty}{\infty}$

人र

$\sqrt{1 / 2}$

N

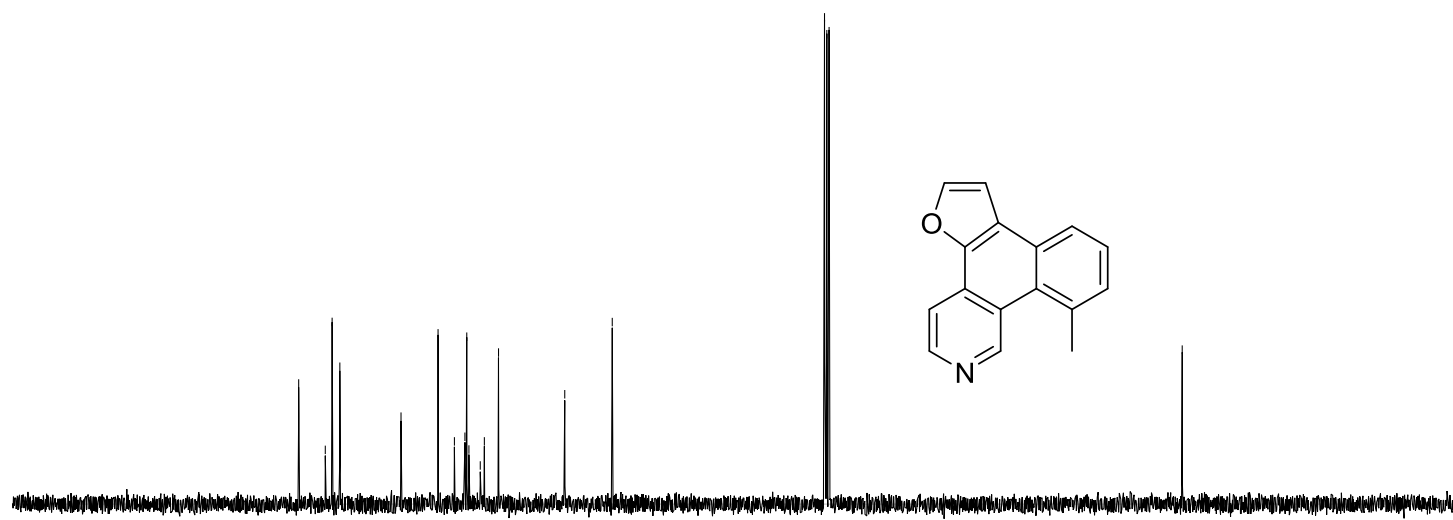

90

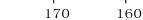

$150 \quad 140$

f1 $\stackrel{90}{(\mathrm{ppm})}$ 


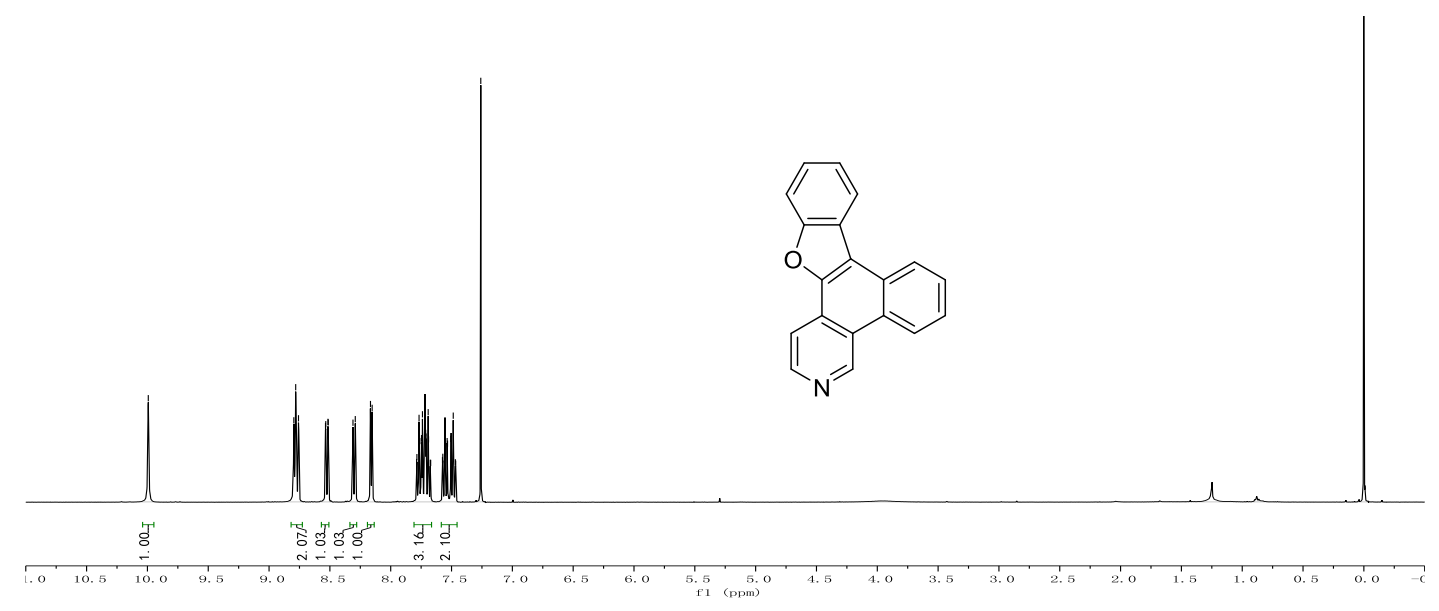

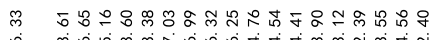

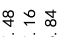

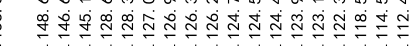

î́5

Ti, 年

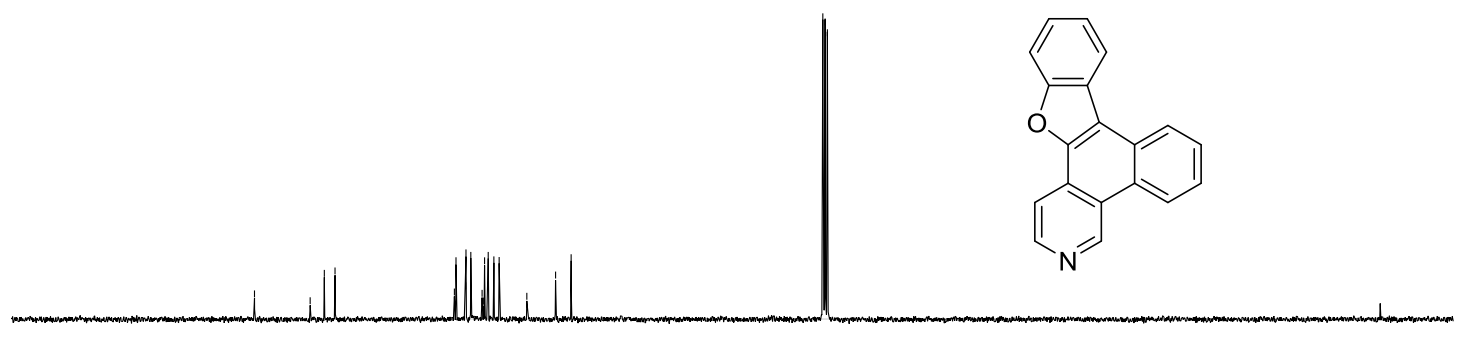


(6j)

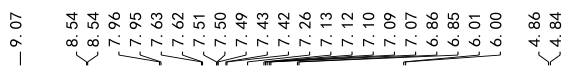

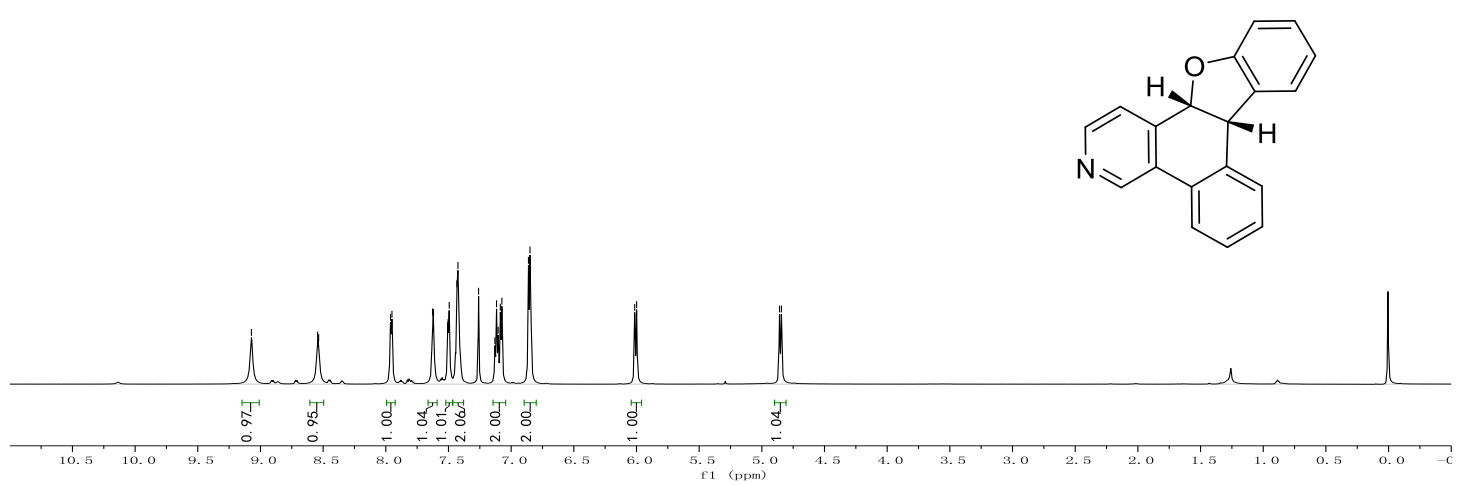

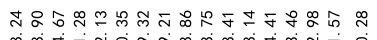

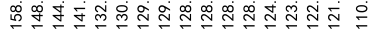
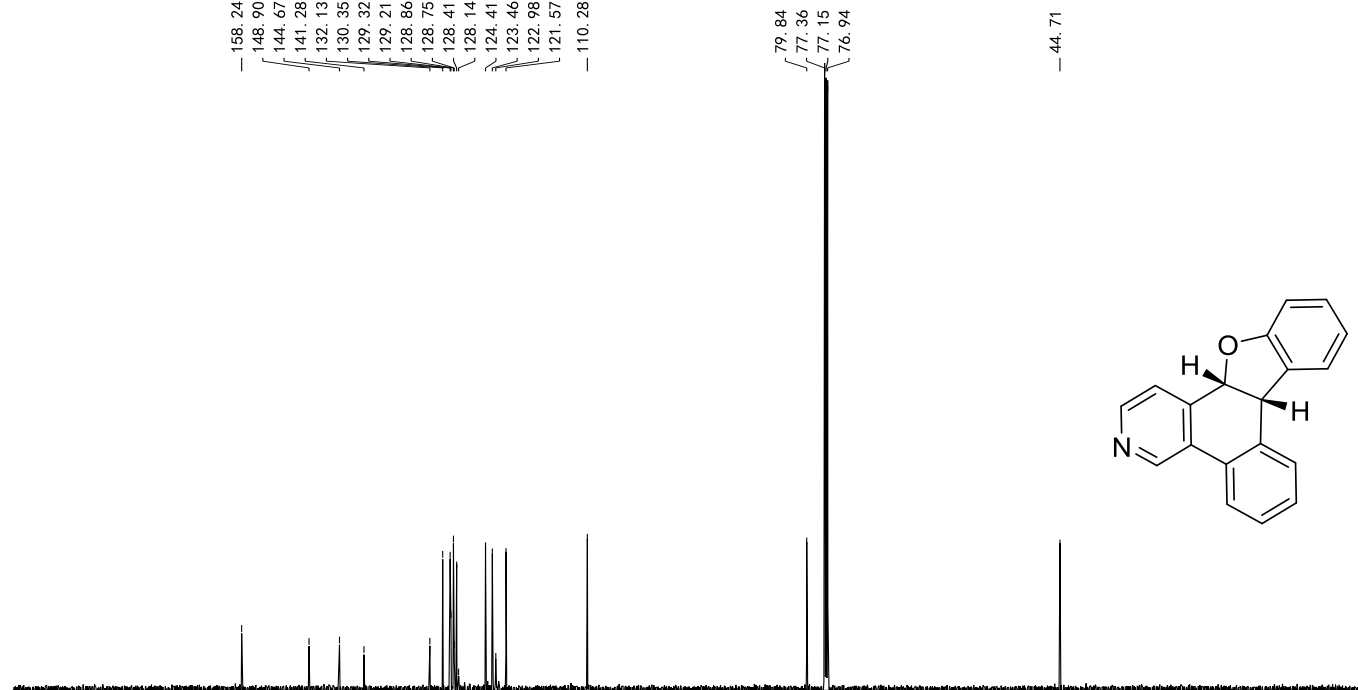

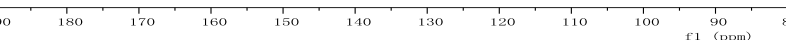


(8a)

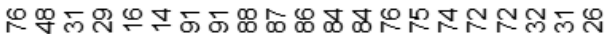

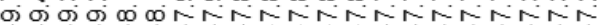

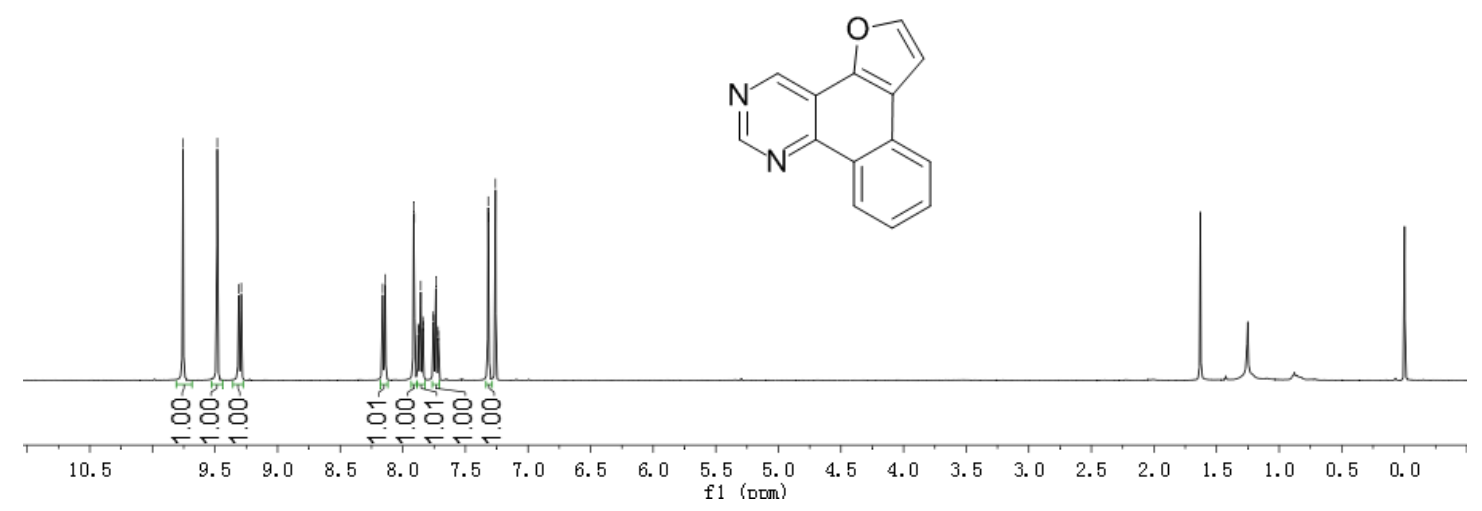

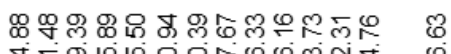

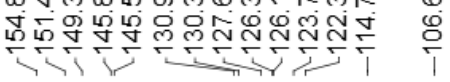

事辑
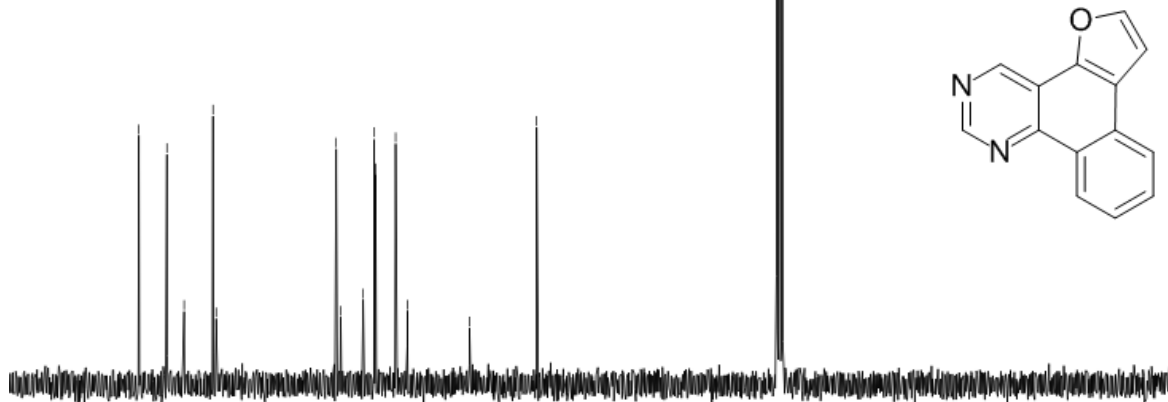

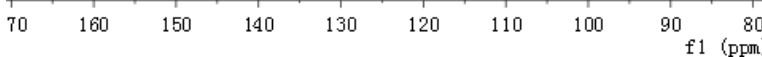




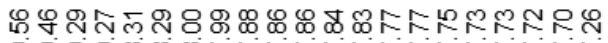

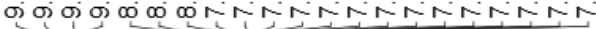
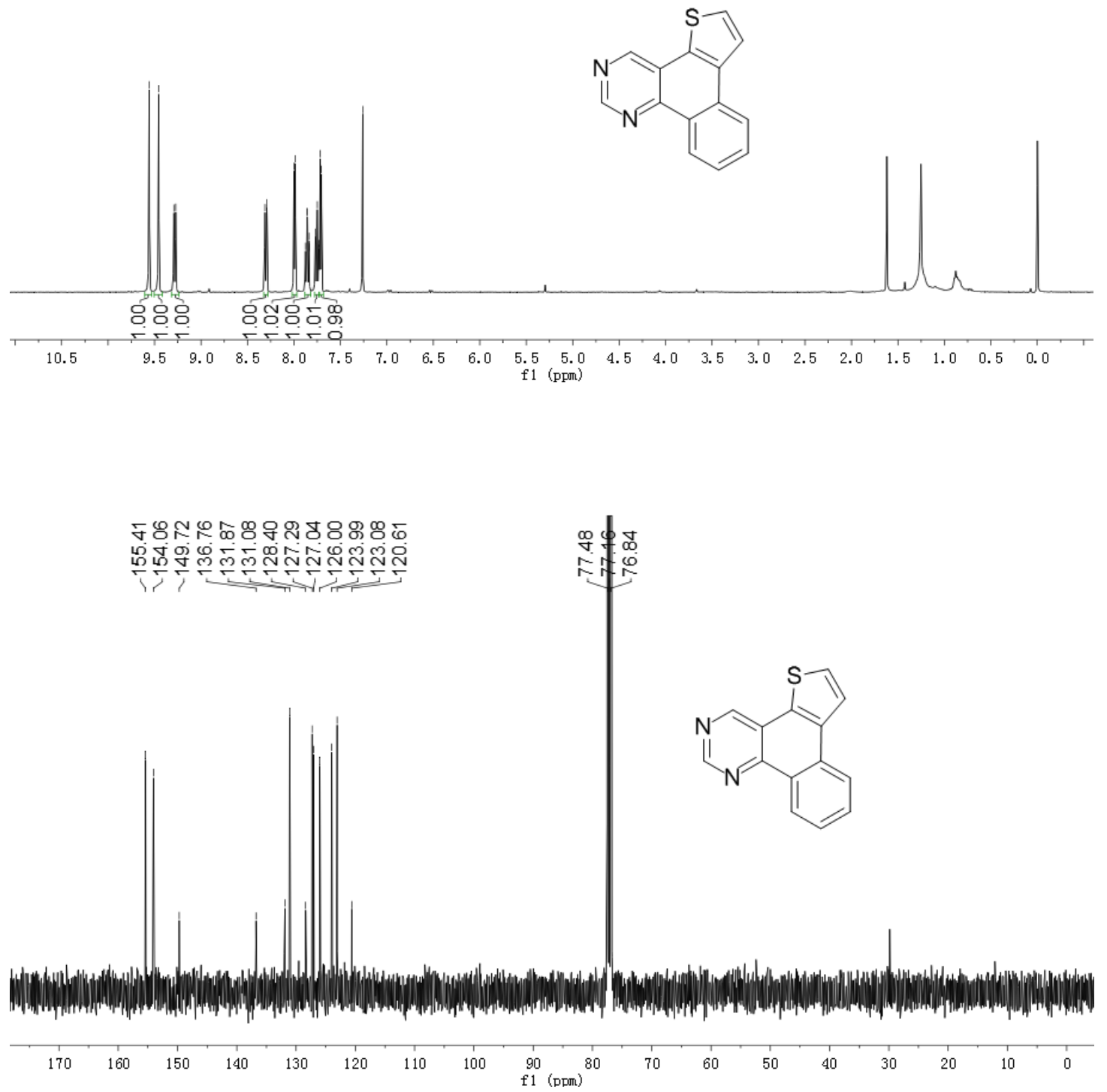
(8c)

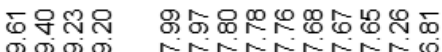

NNNNNNE

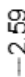

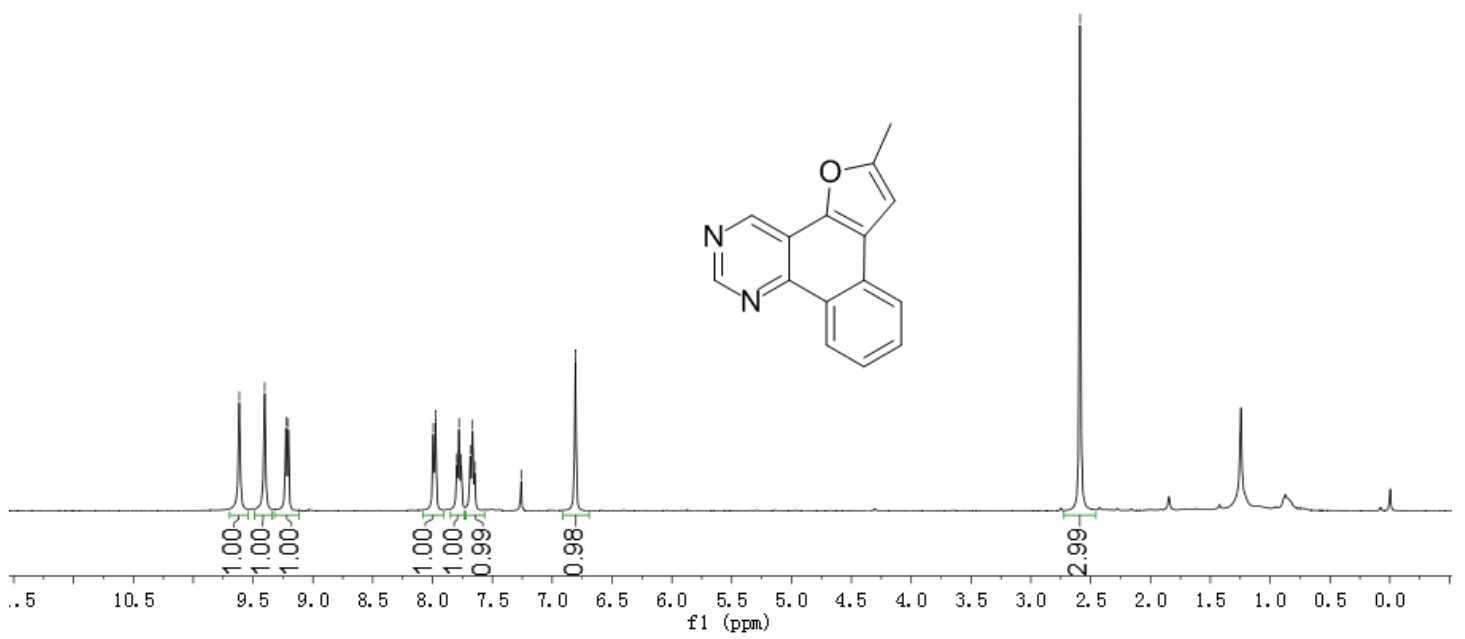

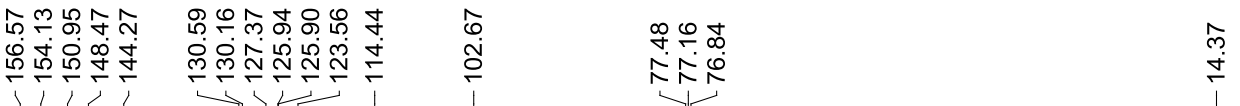

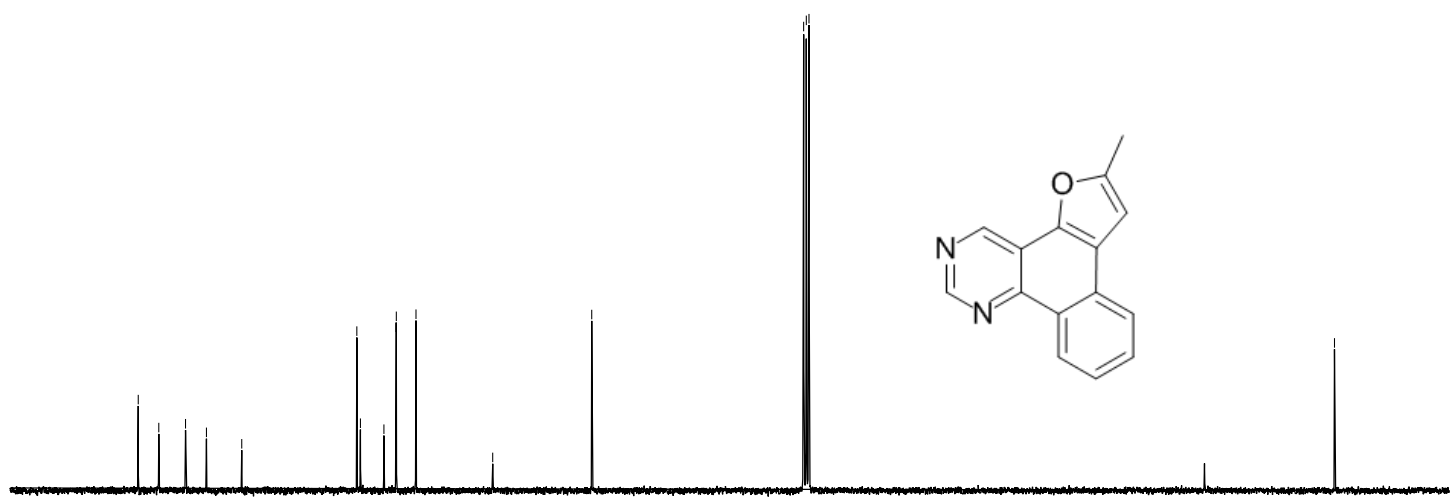

$90 \quad 80$ 
(8d)

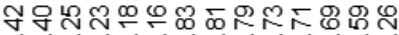

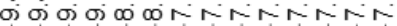

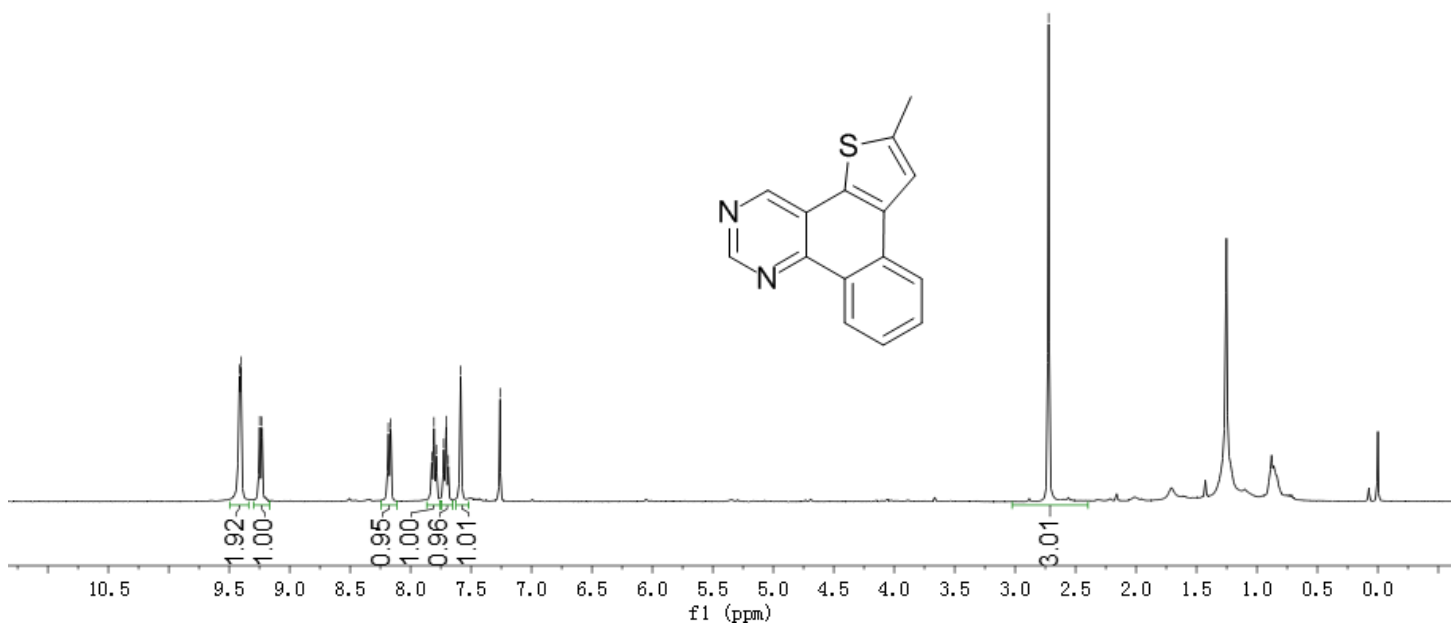

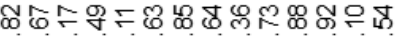

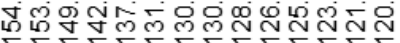

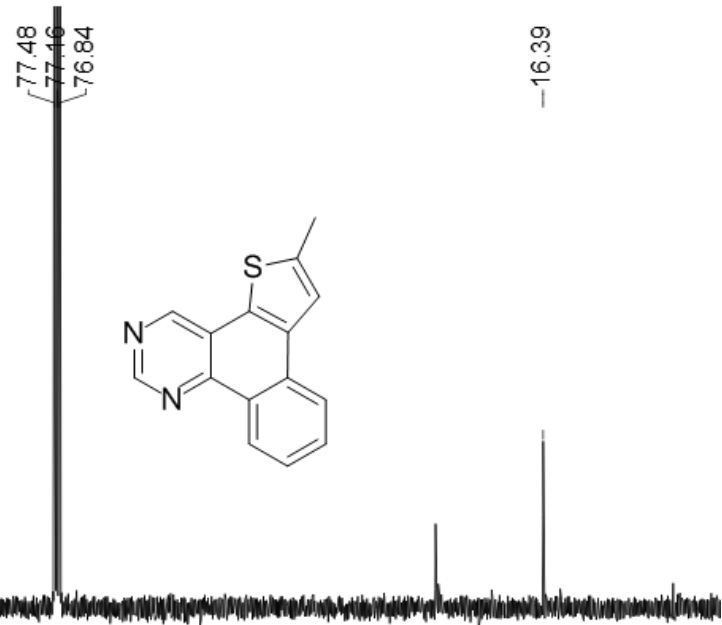

